This document is confidential and is proprietary to the American Chemical Society and its authors. Do not copy or disclose without written permission. If you have received this item in error, notify the sender and delete all copies.

\title{
Colloidal Nanocrystals as Electrocatalysts with Tunable Activity and Selectivity
}

\begin{tabular}{|c|c|}
\hline Journal: & ACS Catalysis \\
\hline Manuscript ID & cS-2020-04403f.R1 \\
\hline Manuscript Type: & Review \\
\hline $\begin{array}{r}\text { Date Submitted by the } \\
\text { Author: }\end{array}$ & $\mathrm{n} / \mathrm{a}$ \\
\hline Complete List of Authors: & $\begin{array}{l}\text { Guntern, Yannick; Ecole polytechnique federale de Lausanne Faculte des } \\
\text { sciences de base, Department of Chemical Sciences and Engineering } \\
\text { Okatenko, Valery; Ecole polytechnique federale de Lausanne Faculte des } \\
\text { sciences de base, Department of Chemical Sciences and Engineering } \\
\text { Pankhurst, James; Ecole polytechnique federale de Lausanne Faculte des } \\
\text { sciences de base, Department of Chemical Sciences and Engineering } \\
\text { Varandili, Seyedeh Behnaz; Ecole polytechnique federale de Lausanne } \\
\text { Faculte des sciences de base, Department of Chemical Sciences and } \\
\text { Engineering } \\
\text { Iyengar, Pranit; Ecole polytechnique federale de Lausanne Faculte des } \\
\text { sciences de base, Department of Chemical Sciences and Engineering } \\
\text { Koolen, Cedric; Ecole polytechnique federale de Lausanne Faculte des } \\
\text { sciences de base, Department of Chemical Sciences and Engineering } \\
\text { Stoian, Dragos; EPFL, LNCE } \\
\text { Vavra, Jan; Ecole polytechnique federale de Lausanne Faculte des } \\
\text { sciences de base, Department of Chemical Sciences and Engineering } \\
\text { Buonsanti, Raffaella; Ecole polytechnique federale de Lausanne Faculte } \\
\text { des sciences de base, Department of Chemical Sciences and Engineering }\end{array}$ \\
\hline
\end{tabular}

\section{SCHOLARONE ${ }^{\text {m }}$ Manuscripts}




\title{
Colloidal Nanocrystals as Electrocatalysts with
}

\section{Tunable Activity and Selectivity}

\author{
Yannick T. Guntern, ${ }^{\dagger}$ Valery Okatenko, ${ }^{\dagger}$ James Pankhurst, ${ }^{\dagger}$ Seyedeh Behnaz \\ Varandili,${ }^{\dagger}$ Pranit Iyengar, ${ }^{\dagger}$ Cedric Koolen, ${ }^{\dagger}$ Dragos Stoian, ${ }^{\dagger}$ Jan Vavra, ${ }^{\dagger}$ Raffaella \\ Buonsanti†* \\ $\dagger$ Laboratory of Nanochemistry for Energy (LNCE), Department of Chemical Sciences and \\ Engineering, École Polytechnique Fédérale de Lausanne, CH-1950 Sion, Switzerland. \\ * Corresponding author: raffaella.buonsanti@epfl.ch
}

\begin{abstract}
Correlating the catalyst activity, selectivity and stability with its structure and composition is of the utmost importance in advancing the knowledge of heterogeneous electrocatalytic processes for chemical energy conversion. Well-defined colloidal nanocrystals with tunable monodisperse size and uniform shapes are ideal platforms to investigate the effect of these parameters on the catalytic performance. In addition to translate the knowledge from single crystal studies to more realistic conditions, the morphological and compositional complexity attainable by colloidal chemistry can provide access to active catalysts which cannot be produced by other synthetic approaches. The sample uniformity is also beneficial to investigate catalyst reconstruction processes via both ex-situ and operando techniques. Finally, colloidal nanocrystals are obtained as inks, a feature which facilitates their integration on different substrates and cell configurations to study the impact of interactions at the mesoscale and device-dependent reaction microenvironment on the catalytic outcome. In this Review, we discuss recent studies in selected electrochemical reactions and provide our outlook on future developments on the use of well-defined colloidal nanocrystals as an emerging class of electrocatalysts.
\end{abstract}




\section{Introduction}

As the production of renewable energy increases and electricity prices go down, energy storage becomes a must to move towards a sustainable society. While batteries are one of the best options for short-term energy storage, the possibility of storing energy in chemical bonds is appealing for long-term storage. In this context, electrolyzers and fuel cells have become the major technologies. Electrocatalysts are a key component of these devices as they facilitate the conversion of electrical energy into chemical energy and vice versa. Correlating the catalyst activity, selectivity and stability with its structure and composition is of the utmost importance in advancing the knowledge of chemical transformations and eventually improving device performance.

Towards this aim, one approach is to study industrial catalysts with the advanced characterization methods available nowadays, i.e. at synchrotron and microscopy facilities. ${ }^{1}$ Generally, such catalysts consist of particles with large size distributions spanning from $1 \mathrm{~nm}$ to $100 \mathrm{~nm}$ and various morphologies, often mixed or embedded in a support, that can contain carbon, ceramic materials and/or polymeric binders. This heterogeneity makes it non-trivial to identify the catalyst features that determine its behavior.

A second approach is to prepare materials with well-defined and tunable composition, size and shape that serve as model systems to understand the effects of these parameters on the catalyst functionality. ${ }^{2-8}$ These materials span from single crystals, to clusters deposited using ultrahigh vacuum techniques, to nanoparticles prepared via different chemical routes (i.e. sol-gel, dissolution-reprecipitation, impregnation). ${ }^{2-7,9}$ Among wet-chemistry techniques, colloidal methods stand out because of the superior control of nanocrystal composition and morphological features. ${ }^{2-4}$

In this review, we highlight the contribution of well-defined colloidal nanocrystals (NCs) in advancing the understanding of electrocatalytic reactions that are relevant for energy storage in chemical bonds. Before diving into the selected reactions, we briefly describe the synthesis of colloidal NCs. Then, we present various deposition techniques of the colloidally stable NC inks on the conductive substrates used for the electrocatalytic measurements. Only studies employing catalysts synthesized according to the described colloidal approach and obtained as dispersible NCs with monodispersed sizes and uniform shapes are reviewed later. While essential during the synthesis and to assure solvent dispersibility of the NCs, surface ligands are normally considered to poison the catalyst surface. We review various methods for their removal before moving on to describe the electrochemical cells most commonly used to 
evaluate the NC electrocatalytic performance. Following this first part, we focus on the reactions of interest: the hydrogen evolution and oxidation reactions (HER/HOR), the oxygen evolution and reduction reactions (OER/ORR), the $\mathrm{CO}_{2}$ reduction reaction $\left(\mathrm{CO}_{2} \mathrm{RR}\right)$, the formic acid and various alcohol oxidation reactions (FAOR, methanol (MOR), ethylene glycol (EGOR), ethanol (EOR) and glycerol (GOR)). Other electrochemical reactions, such as the nitrogen reduction reaction, are not covered as the examples including colloidal NCs as the catalysts are very few or none at all. When possible, we have prioritized representative case studies (i.e. metal phosphide NCs for HER, nickel-platinum NCs for ORR, copper-based NCs for $\mathrm{CO}_{2} \mathrm{RR}$ ) to provide more direct evidence of the progress made possible by the systematic tuning of catalyst size, shape, and composition that is enabled by colloidal chemistry. A section dedicated to interfacing NCs with building blocks of different chemical nature (organic ligands, carbon materials, polymers, metal-organic frameworks) is included as these nanocomposites have been shown to possess interesting synergistic properties in many instances. After, we discuss selected examples which highlight how colloidal NCs, well-defined in size and shape, have been used specifically to understand structural changes under/post reaction conditions before providing an overview of the in situ and operando tools specific for colloidal NC catalysts. Finally, we elaborate on some of the open challenges, before concluding with an outlook on future opportunities offered by atomically-tunable colloidal NCs in electrocatalysis.

\section{Colloidal synthesis of $\mathrm{NC}$ catalysts}

A typical colloidal synthesis involves the reduction or decomposition of metal precursors in the presence of surfactants in either aqueous or organic media (Figure 1). ${ }^{10-17}$ Surfactants are crucial for tuning the NC size and shape which they contribute to by modulating the free energy landscape when taking part in the reaction, and/or by acting as surface passivating agents. ${ }^{10-17}$ In addition to the ligands, many reaction parameters such as temperature, concentrations, atmosphere, injection rates, and reactor volume need to be carefully tuned during the synthesis, in order to tailor-make NCs at will. ${ }^{10-16}$

The synthesis of noble metal NCs is most commonly performed in aqueous environment. ${ }^{10,11}$ Many procedures are based on the reduction of metal salts by a polyol, usually ethylene glycol (EG) or diethylene glycol, which is also the solvent, in the presence of a capping agent, such as polyvinylpyrrolidone (PVP), citrate or halogen ions. EG possesses three key features, which explain its large use: it dissolves most of the metal salts; it has a high boiling point, which 
provides flexibility in varying the reaction temperature; its reducing power is temperature dependent, so one can easily tune the monomer flux, which is an important parameter for shape control. ${ }^{10,11}$ Other reducing agents used across the literature include L-ascorbic acid, citric acid and PVP itself. ${ }^{10,11}$ Their different reduction power results in different reduction rates, thus, different shapes can be obtained, accordingly. ${ }^{10,11}$

The synthesis of non-noble metal NCs is certainly less mature in terms of size and shape control. ${ }^{17}$ One of the reasons behind this delayed development is the tendency of non-noble metals to easily oxidize, restraining the choice only to strongly reducing agents when the synthesis is carried out in aqueous environment. The use of a strong reducing results in the formation of very small particles and reduces the diversity of sizes and shapes attainable. Alternatively, the synthesis can be conducted in organic solvents, however in such environment the knowledge about the formation mechanism of metal NCs is still in progress. ${ }^{15,16}$ With the exception of $\mathrm{Cu}$, non-noble metal NCs have been mostly obtained as nanospheres or nanorods. ${ }^{17}$

Binary metal oxide NCs, such as iron oxide, titanium dioxide, tungsten oxide, have been mostly synthesized via decomposition of metal acetylacetonates, acetates, carboxylates precursors in the presence of organic ligands, the most common being carboxylic acids, amines and diols. ${ }^{12}$ As discuss in the section dedicated to OER/ORR, the synthesis of colloidal multication metal oxide NCs with controlled size and shape remains still an open challenge.

The colloidal synthesis of metal phosphides NCs, including Co-P, Ni-P and Fe-P, has developed substantially in the past 10 years. ${ }^{18}$ Most commonly, it involves the reaction of organometallic precursors or metal nanoparticles with organophosphine reagents, such as trioctlyphosphine. The latter together with amines acts also as the surface ligand.

II-VI NCs (i.e. CdSe, CdS, ZnSe) are the most developed and studied systems. They are normally synthesized using what is referred to as "hot-injection techniques". Here, a highly reactive precursor of the anion (i.e. dimethyl cadmium) is injected in a high boiling point solvent (i.e. octadecene or trioctlyphosphine oxide) in the presence of a chalcogenide source (i.e. trialkyl phosphine selenide. ${ }^{19-21}$ Phosphines and thiols are the most common ligands, but there are many examples also with amines and carboxylates. It is noted that the colloidal synthesis of well-defined and phase controlled 2D transition metal chalcogenides and phosphides remains a challenge and only a few examples are reported in the literature. ${ }^{22,23}$ Therefore, while interesting for some of the selected reactions, especially HER, these classes of materials are not included in the following sections. 
For all the above classes of materials, the level of complexity further expands by using the seed-mediated growth, wherein pre-synthesized NCs are utilized as nuclei for the deposition of a second material with the same or different composition. ${ }^{10,11,14}$ The purpose of this method is to utilize the seeds to direct the shape of the growing domain, thus enabling access to more complex shapes. This principle has been utilized heavily for noble metal NCs. ${ }^{10,11}$ Another goal might be to synthesized nanocrystalline heterostructures, where NC domains of different composition are intimately linked by an interface. ${ }^{14}$ The morphology of such heterostructures (i.e. core@shell, dimers, dumbells) depends on various factors, including lattice mismatch, precursor reactivity and miscibility of the two domains. ${ }^{14,24-28}$

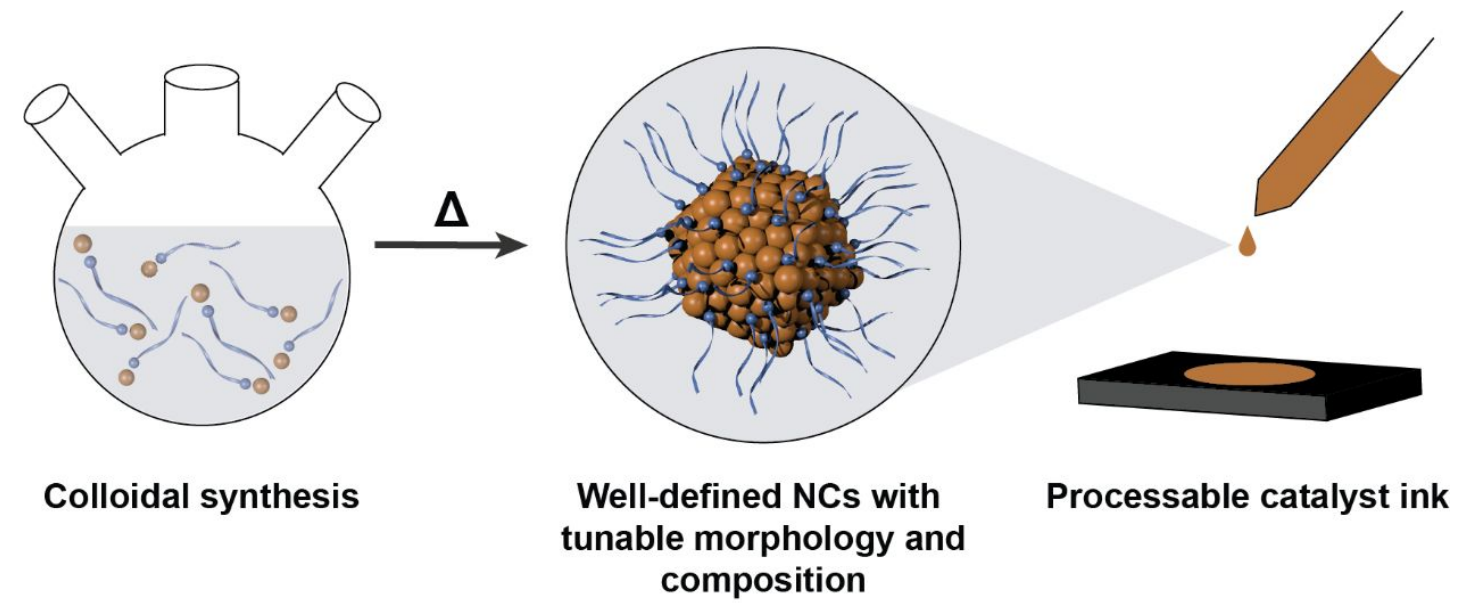

Figure 1. Scheme illustrating the colloidal synthesis of well-defined nanocrystals (NCs).

\section{Deposition techniques for colloidal NC catalysts}

One big advantage of NCs prepared via colloidal routes is that they usually come dispersed in a solution of organic apolar solvents (i.e. hexane, toluene, octane) (Figure 1). This NC ink can then be easily deposited on different substrates using drop-casting, spin-coating and spraycoating. ${ }^{29}$ While utilized in some studies, the addition of additives, such as Nafion or other polymers, that is crucial for powder catalysts, is not needed for colloidally synthesized NCs to adhere on the electrodes.

Drop-casting is the simplest technique, as it does not require sophisticated experimental setups and is well suited for any type of substrate. Here, the catalyst ink is deposited onto the electrode surface as a droplet and the solvent is left to evaporate. ${ }^{29}$ On flat substrates, very uniform NC 
monolayers, where the spacing is tunable via the aliphatic chain of the ligands, can be obtained by properly controlling the solvent properties and the evaporation environment. ${ }^{30,31}$

Spin-coating is another method often used for the deposition of colloidal NC catalysts, although it is limited to flat substrates, such as glassy carbon electrodes. ${ }^{29}$ The latter are rotated in a spincoater which controls the process parameters (i.e. rotation speed, dwelling time). Spin-coating provides more control over the uniformity of the NC films compared to drop-casting by tuning the rotation speed, solution viscosity and NC concentration. Sparse or dense NC monolayers can be obtained. It is also suitable for the preparation of thick multilayered films if needed. The main issue with spin coating is that a large volume of solution is wasted during the process, and uniform deposition over large areas is not possible.

Spray-coating, or air-brushing, is a very useful technique to deposit NC inks on large areas of any substrate and it is particularly helpful when building gas diffusion electrodes (GDEs), as it is not sensitive to the substrate surface morphology. ${ }^{32}$ Here, the NCs are deposited while the solvent evaporates. By controlling the solution viscosity and concentration, spraying intensity (droplets size and speed) and spraying geometry (tip geometry, distance between the tip and the substrate), the process can be controlled to obtain samples with desired properties. With the use of electro-spraying, the deposition can be controlled to even further extent. ${ }^{33,34}$ More generally, having the variety of parameters to control the deposition is a benefit, as it allows tunability, but also a drawback, as it can be challenging to control them between subsequent depositions and can result in reproducibility issues. Thus, a fully automated system for spray coating is advisable.

Generally, one can choose the method which is the most appropriate for the utilized substrate. Furthermore, utilizing different deposition methods can be regarded as a valuable tool to control the catalyst properties, including local reaction environment and mass transport conditions or hydrophobicity when the catalyst is mixed with other components. To cite just one example, Garcia de Arquer et al. were able to obtain both planar and bulk catalyst:ionomer heterojunctions thanks to the precise control over the deposition procedures. ${ }^{35}$ Indeed, while only a planar heterojunction was formed when spray-coating the ionomer onto the magnetron sputtered $\mathrm{Cu}$ catalyst layer, the authors were able to create the electrode with bulk catalyst:ionomer heterojunction by spraying the mixture with carefully tuned ratio between perfluorinated sulfonic acid ionomer (Nafion) and $\mathrm{Cu}$ NCs in methanol. As a result, thanks to the presence of both hydrophilic and hydrophobic moieties in the ionomer, an electrode architecture that decouples gas, ion, and electron transport was created, with the mass transport 


\section{Removal of surface ligands}

Organic ligands are critical to the synthesis of colloidal NCs, yet they are often viewed as problematic with regards to catalysis, as they might block active sites on the catalyst surface and hinder the approach of reactants. For this reason, ligands are often removed from the NC surface prior to catalysis.

As illustrated in Figure 2, a number of different approaches to remove ligands from NC surfaces have been developed, including thermal, plasma, chemical and electrochemical treatments. ${ }^{41-44}$

Thermal methods decompose organic ligands and it is often assumed that the volatile residues from these ligands are swept away in the gas phase. Particular care should be taken in choosing the annealing conditions to ensure that they modify neither the original composition nor the original size and morphology of the NC catalysts. Generally, mild annealing is used with temperatures below $200{ }^{\circ} \mathrm{C} .45,46$ Cargnello et al. have demonstrated that fast thermal treatments $\left(700^{\circ} \mathrm{C}\right.$ for $30 \mathrm{~s}$ ) can also efficiently remove the ligands while preserving the size and shape of oxide-supported metal NCs. ${ }^{47}$ Calcination has proven effective in activating HER, OER/ORR, $\mathrm{CO}_{2} \mathrm{RR}$ and MOR NC electrocatalysts. ${ }^{45,46,48-53} \mathrm{Lu}$ et al. showed a direct correlation between ligand surface coverage of Au NCs and ORR performance. ${ }^{54}$ Likewise, Li et al. demonstrated 
that mild annealing $\left(185^{\circ} \mathrm{C}\right)$, was more effective than other treatments, including UV-ozone and acid treatments, to remove oleylamine ligands from Pt ORR catalysts. ${ }^{46}$ However, high temperatures can also cause sintering and restructuring of $\mathrm{NC}$ catalysts, as well as changes in surface composition, which can depend on the environment used. ${ }^{55}$ Work by Mohapatra et al. also highlighted that calcination of $\mathrm{NC}$ assemblies can induce cracking in the film, and that poorly defined carbonaceous residues remain. ${ }^{56}$ The authors advocate for plasma cleaning to be a much more effective method to remove carbon material without damaging the NC films and in order to create a conductive network.

Plasma-cleaning can make use of a variety of gases, with air, $\mathrm{O}_{2}, \mathrm{H}_{2}, \mathrm{~N}_{2}, \mathrm{He}$ or Ar being some common examples. ${ }^{56-61} \mathrm{O}_{2}$ plasma is particularly effective at removing ligands as they are broken down into volatile oxygenated species. However, metallic NCs can also be oxidized under such conditions, which will significantly alter the electrocatalyst properties. Work led by Roldan Cuenya has demonstrated that this can be beneficial in $\mathrm{CO}_{2} \mathrm{RR}$ catalysis, as the surface and subsurface copper-oxygen species can boost $\mathrm{C}_{2}$-selectivity. ${ }^{60,62} \mathrm{Gehl}$ et al. showed that the metallic state of Pt NCs can be reestablished with a subsequent reductive plasma treatment $\left(\mathrm{N}_{2}\right.$ or $\mathrm{H}_{2}$ ), although Co NCs still retained some surface-oxygen species. ${ }^{59}$ In our own studies, we have opted for $\mathrm{N}_{2}$ plasma to remove ligands while preserving cubic and octahedral $\mathrm{Cu} \mathrm{NC}$ morphologies and the metallic state of the $\mathrm{CO}_{2} \mathrm{RR}$ catalyst. ${ }^{63,64} \mathrm{He}$ plasma holds many benefits in ligand removal in that it is chemically inert and is also non-ablative, and is therefore compatible with soft materials. ${ }^{61}$ It should also be noted that plasma methods can still pose structural issues, such as sintering and cracking of NC films, depending on the conditions used. ${ }^{65}$

Chemical oxidation by ozone and UV/ozone treatments are similar to plasma methods in that oxidative removal of ligands can be very effective, but can also leave the metal surface partially oxidized. ${ }^{66-68}$

Chemical treatments of NC surfaces are much milder compared to thermal or plasma techniques, and can offer superior control over the surface composition. In the simplest case, ligands can be removed by repeated solvent washing. ${ }^{69-71}$ Similarly, vacuum can be enough to remove ligands if the $\mathrm{NC}$-ligand bond is not strong and if the ligand is sufficiently volatile. ${ }^{72}$ Organic ligands can be displaced by small anions, such as hydroxide or hydrides, which impose less steric restrictions on the activity. ${ }^{73-75}$ The result in this case is that the NCs are protected by small anionic adsorbates. ${ }^{76}$ Hydrazine is a common reagent used for ligand removal. For example, Zhang et al. used this reagent to remove trioctylphosphine (TOP) ligands from Bi particles, which both stripped the ligands from the surface and reduced the surface $\mathrm{Bi}^{3+}$ species, 
greatly improving the $\mathrm{CO}_{2} \mathrm{RR}$ selectivity and activity. ${ }^{77}$ In that case, the role of hydrazine is to act as a Lewis base, displacing the native TOP ligands. In one recent example, Shi et al. demonstrated that hydrazine in trace amounts effectively removes $\mathrm{Br}^{-}$ions from the surface of $\mathrm{Pd}$ nanocubes without modifying their surface structure and subsequently promotes their activity towards the FAOR. ${ }^{78}$

The aim of many chemical stripping methods is to diminish the coordinating ability of the ligand. For example, basic ligands such as amines detach from the surface after protonation by a Brønsted acid. ${ }^{79,80}$ Fan et al. demonstrated how the activity of Pt ORR catalysts was greatly improved by such a ligand-exchange and protonation strategy in comparison with simple ethanol washing. ${ }^{79}$ Lewis-basic ligands can also be detached by forming adducts with Lewis acids such as $\mathrm{BF}_{3} \cdot{ }^{73,81}$ One particularly versatile method is to use alkylating agents, such as Meerwein's salts, $\left[\mathrm{R}_{3} \mathrm{O}\right]\left[\mathrm{BF}_{4}\right]$, which introduce a small alkyl group to the coordinating atom on the ligand, which then detaches from the surface. ${ }^{73,82-84}$ To cite one example, Henckel et al. used such an approach to remove amine ligands from $\mathrm{WSe}_{2} \mathrm{NCs}^{82}$ They demonstrated that this post-synthetic surface modification represents a simple approach to access electrocatalysts with improved overpotential and intrinsic activity. Nitrosonium salts (e.g. $[\mathrm{NO}]\left[\mathrm{BF}_{4}\right]$ ) have been used in a similar way, where the reactive $\mathrm{NO}^{+}$cation removes the ligands from the surface. In work by Dong et al., nitrosonium-salt treatment of Fe-Pt NCs led to an improvement in the FAOR current density. ${ }^{85}$ In a comparative study by Nelson et al., a series of ligand removal methods on $\mathrm{Mn}_{3} \mathrm{O}_{4} \mathrm{NCs}$ was investigated, and the $\mathrm{NC}$ redox activity decreased following the series: alkylation $>$ Lewis acid $>$ base $>$ heat. ${ }^{73}$ These findings indicate that alkylation methods may be most suitable for activating electrocatalysts.

Electrochemical stripping of the ligands is an attractive approach as it can be carried out postdeposition, just before electrocatalysis takes place. Lu et al. showed that organic ligands can be removed from Pt NCs by applying either anodic or cathodic potentials in water, leading to improved ORR and MOR performance. ${ }^{86}$ Ligand detachment from $\mathrm{Cu}$ and $\mathrm{Au}$ NCs has been demonstrated to occur during the first few minutes of $\mathrm{CO}_{2} \mathrm{RR} \cdot{ }^{63,87}$ Recently, Pankhurst et al. correlated the potential at which ligands are stripped from the surface of $\mathrm{Cu}$ NCs with the metal-ligand bond strength. ${ }^{88}$ Such in situ ligand stripping is appealing as it negates the need for explicit pre-treatment, and challenges the notion that ligands must be removed prior to electrocatalysis in order to attain high current densities. However, further studies to determine the fate of these ligands are needed and care must be taken when cycling the potential in these pre-treatments, as strongly anodic potentials can modify the exposed NC facets. ${ }^{63,89}$ 


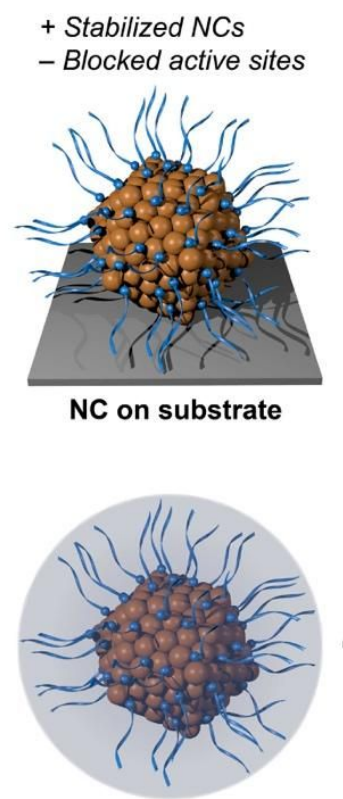

$\mathrm{NC}$ in solution

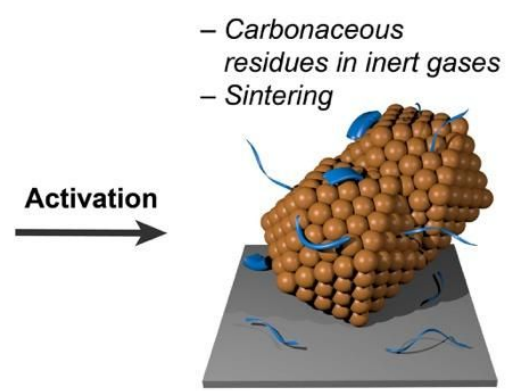

Calcination

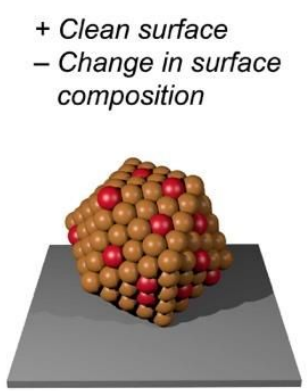

Plasma / ozone

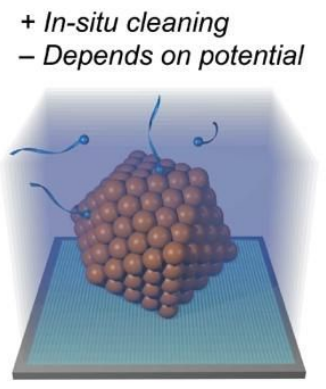

Applied potential

Figure 2. Overview of ligand-removal strategies from NCs deposited on solid supports and in solution; the latter are also applicable post-deposition on substrates. The main advantages and possible drawbacks are listed on top.

\section{Cell types}

Across the literature, different electrochemical cell configurations are employed depending on the aim of the study. In more fundamental studies, experimental setups are carefully designed to determine the intrinsic properties of the catalysts without interference from mass transport limitations. At the same time, industrial use normally implies the current densities to be in the range of $0.1-1 \mathrm{~A} / \mathrm{cm}^{2}$ and often to work in a mass transport limited regime, thus the direct transfer of information from fundamental studies to industrially relevant systems is challenging and a dedicated discussion will come later in the review. These different needs translate into the existence of several cell types, among which the rotating disk electrode (RDE) cells, Hcells, gas-fed flow cells based on GDEs and membrane electrode assembly (MEA) cells will be covered in this section..$^{90}$ However, it is worth noting that the cell configurations are not limited to these types only. For example, one can refer to differential electrochemical massspectrometry (DEMS) or online cells, which are specialized microfluidic cells for in situ mass resolved determination of gaseous or volatile electrochemical reactants, reaction intermediates and products in real time. ${ }^{91-93}$ 
The RDE is normally implemented in a three-electrode flask setup and is used intensely, particularly in fundamental studies (Figure 3A). Here, the catalyst is deposited by dropcasting onto the flat surface of the electrode rod, typically made of glassy carbon or noble metals such as $\mathrm{Pt}$ or $\mathrm{Au}$. For this setup, the hydrodynamic equations and the convective-diffusion equation have been solved rigorously for the steady state, thus making it possible to extract the intrinsic performance metrics of the catalyst. ${ }^{94}$ However, the current magnitude is limited to few microamperes, due to the small electrode surface area (around $0.1 \mathrm{~cm}^{2}$ ) and to the low catalyst loading. In general, the direct transfer of information from RDE studies to commerciallyrelevant systems is not straightforward and will be discussed later.

The H-type cell is another common setup (Figure 3B). It contains two compartments (anodic and cathodic) separated by an ion-exchange membrane. Here, the NC catalysts are typically supported on glassy carbon or carbon cloth/paper, metallic electrodes (e.g. Ti) etc., being used with geometric surface areas between $1 \mathrm{~cm}^{2}$ and $2.5 \mathrm{~cm}^{2}$. Information about the intrinsic properties of $\mathrm{NC}$ catalysts can still be obtained in this configuration, and H-cells have been used for most of the $\mathrm{CO}_{2} \mathrm{RR}$ systems discussed below. Nevertheless, it is important to perform the measurements in the absence of mass transport effects and without introducing artifacts of any kind. ${ }^{95}$ While H-cells have a less defined mass transport compared to RDEs, they afford larger electrode areas which are more suitable for product detection, which is extremely important to evaluate catalyst selectivity in $\mathrm{CO}_{2} \mathrm{RR}$. At the same time, the conditions under which the catalysts operate (i.e. current density, local microenvironment, mass transport) are still far from those of a commercial electrolyzer. ${ }^{96,97}$ 

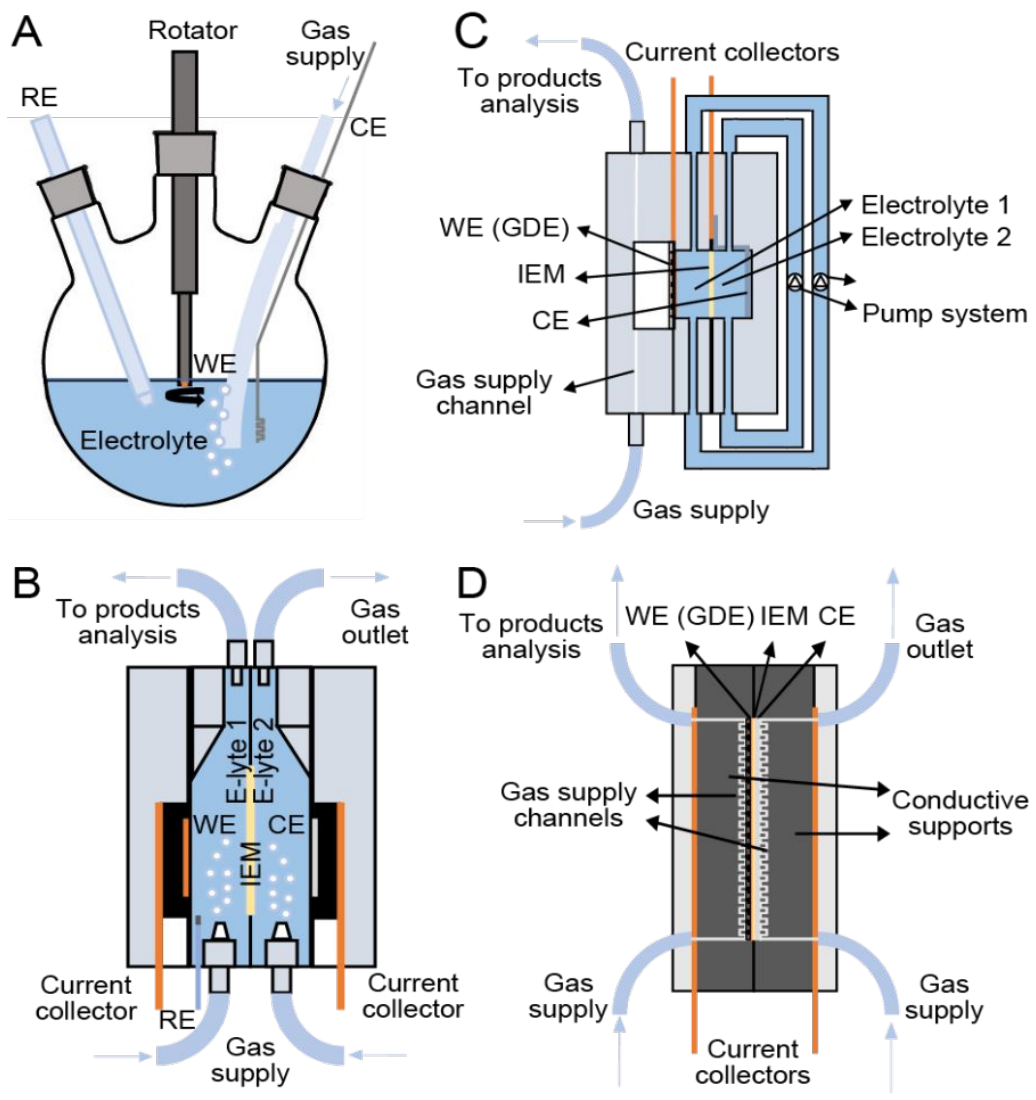

Figure 3. Representative schemes of (A) RDE, (B) H-cell, (C) gas-fed flow-cell including and (D) MEA cell setups.

Gas-diffusion electrodes (GDEs) consist of a porous layered carbon structure and are the substrates of choice for both gas-fed flow cells and MEA cells. ${ }^{96-101}$ In a gas-fed flow cell, a liquid electrolyte is still used (Figure 3C). Here, the GDE is backed by a gas chamber and faced to the corresponding electrolyte compartment (e.g., the catholyte for $\mathrm{CO}_{2} \mathrm{RR}$ ). The catholyte and anolyte chambers are separated by an ion-exchange membrane. The electrolyte is normally pumped through the system in a way similar to flow batteries, so that its composition is more constant throughout the measurements. All record efficiencies in $\mathrm{CO}_{2} \mathrm{RR}$ (i.e. high current densities $>1 \mathrm{~A} \mathrm{~cm}^{-2}$ together with faradaic efficiencies of $45 \%$ towards $\mathrm{C}_{2+}$ products) ${ }^{35}$ have been so far obtained using this type of cell. While testing of NC catalysts on GDEs is still rare, the existing studies reviewed later evidence that colloidal NC catalysts are suitable for implementation into a commercial device. Moreover, they emphasize the importance of exploiting synergisms between catalyst design and system engineering to explore activity, selectivity and stability of nanocatalysts. 
In a MEA cell, the GDE is fed with humified gas on one side and faces directly the ionexchange membrane on the other side (Figure 3D). A device that operates completely in gas phase enables the transition from single cells to cell stacks, which is beneficial for industrial applications as it enables to achieve higher current densities. ${ }^{99,102,103}$ This technology is especially mature in the field of proton exchange membrane (PEM) fuel cells and recent works have been focusing on the implementation of Pt-based NC catalysts in such devices, a topic which is reviewed in a later section.

\section{HER/HOR}

Hydrogen is one of the cleanest alternatives to fossil fuels because of its zero-emissions when reacted with oxygen. Water electrolysis is one valuable option to produce it in a sustainable manner via the hydrogen evolution reaction (HER) at the cathode. The fundamentals of the HER and strategies toward the fabrication of active HER electrocatalysts have been subjects of intense study over the past decades. ${ }^{104}$ The opposite reaction, namely the hydrogen oxidation reaction (HOR), is also extremely important because crucial for the development of PEM fuel cells, which are the best candidates to replace the current alkaline fuel cell technology. ${ }^{105}$ One of the main challenges for the HER/HOR is that platinum group metals (PGMs) possess the best activities, in agreement with the theoretical volcano plot (Figure 4). ${ }^{106}$ Therefore, most research efforts have been directed towards the discovery of catalysts with lower PGM content or based on non-noble metals. To note that in acidic conditions $\mathrm{Pt}$ is extremely active, much more than in alkaline environment, which is due to $\mathrm{pH}$ effects on the $\mathrm{H}$ binding energy. ${ }^{107}$ This difference implies that finding alternative to PGM catalysts in acids is very challenging. On the other side, if one considers that on the OER/ORR side non-noble metal catalysts are very active in alkaline conditions, the discovery of active catalysts in such environment is critical for a PEM technology based on non-noble metals. ${ }^{107}$ Unfortunately, one problem that emerges in non-noble metal catalysts is their stability. ${ }^{108}$ Here, binary compositions including phosphides, carbide and sulfides could help as it is also evident from some of the example below. ${ }^{108}$

Below we discuss studies from the literature, organized by classes of materials, which utilize well-defined NCs as electrocatalysts for HER; studies for HOR are rare. The data are summarized in Table S1. Overall, the main contribution of colloidal chemistry to HER is provided by the systematic tuning of catalyst size, shape, and composition achieved in the metal 
phosphides. Here, the degree of structural and compositional control together with the consistency in the testing conditions under acidic conditions has enable conclusions regarding the impact of these parameters on HER, which are detailed in the related section below. On the other hand, the reported heterostructures based on metal chalcogenides reveal new strategies to improve the catalytic performance of PGMs especially in alkaline conditions.

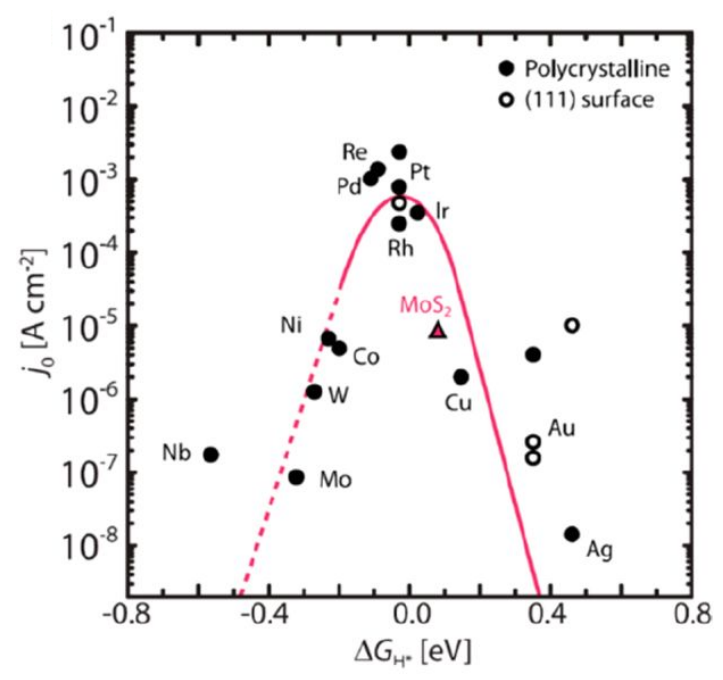

Figure 4. Volcano plot reporting the current density $\left(j_{0}\right)$, as a measure of the HER/HOR catalyst activity, versus the Gibbs free energy for atomic hydrogen adsorption $\left(\Delta \mathrm{G}_{\mathrm{H}^{*}}\right)$ on the surface of various metals in acidic medium. Adapted with permission from reference 106. Copyright 2017, AAAS.

\subsection{Metals}

A few studies on colloidally synthesized metallic NCs have been reported in the literature. ${ }^{109-}$ 115 While remaining a bit scattered, the data available on this class of materials do reveal the potential of well-defined NCs to explore various strategies in metal alloys directed towards reducing the overpotential of PGM-free catalysts or of catalysts with reduced Pt content in both acidic and alkaline conditions. Some of these strategies include increasing the active surface area, modifying the $\mathrm{H}$ binding energy through strain or exposing facets with higher catalytic activity. ${ }^{109-115}$ As mentioned above, to find HER catalysts active in alkaline conditions is critical, therefore we cite a couple of examples relative to this topic. In the first one, McEnaney et al. proposed colloidally synthesized Co-Mo alloyed NCs of $3 \mathrm{~nm}$ as a promising Earthabundant catalyst. ${ }^{114}$ The authors reported that the required overpotential of a $\mathrm{Co}-\mathrm{Mo} / \mathrm{Ti}$ electrode is around $-75 \mathrm{mV}$ at $-10 \mathrm{~mA} / \mathrm{cm}^{2}$, which is among the lowest HER overpotentials in alkaline solutions obtained with non-noble metal electrocatalysts. Such results were possible 
thanks to the increase in active surface area due to the small particle size compared to electrochemically deposited catalysts. Moreover, the $\mathrm{Co}-\mathrm{Mo} / \mathrm{Ti}$ catalysts were demonstrated to be highly stable in $1 \mathrm{M} \mathrm{KOH}$ for $18 \mathrm{~h}$. In a second example, Alinezhad et al. grew Pt islands on branched Ni NCs. ${ }^{112}$ They found the compressive strain arising at the interface, which modifies the $\mathrm{H}$ binding energy, to be the reason for the improved HER activity in these nanocatalysts compared to commercial $\mathrm{Pt} / \mathrm{C}$ tested under the same conditions at $\mathrm{pH} 13$ in 0.1 $\mathrm{M} \mathrm{KOH}$. These catalysts possessed a mass activity of around $8 \mathrm{~mA} / \mu \mathrm{g}_{\mathrm{Pt}}$, compared to around $1 \mathrm{~mA} / \mu \mathrm{g}_{\mathrm{Pt}}$ for commercial Pt, and maintained structural stability for 100,000 cycles.

\subsection{Metal phosphides}

Transition metal phosphides have emerged as a valid alternative to $\mathrm{Pt}$ and are interesting especially in terms of stability. ${ }^{18,108}$ Their high activities have been attributed to the complex role of phosphorus, which is responsible for both weak ligand effects on the metal center and cooperative effects. ${ }^{116} \mathrm{R}$. Schaak and co-workers have significantly contributed to this field; we refer to their review dedicated to the topic for a more comprehensive overview on metal phosphide catalysts synthesized via different approaches. ${ }^{18}$ Those synthesized via colloidal chemistry include $\mathrm{Co}, \mathrm{Ni}, \mathrm{Fe}, \mathrm{W}$ and Mo phosphides. The systematic tuning of catalyst size, shape, and composition together with the consistency in the testing conditions under acidic conditions (Table S1) enables conclusions regarding the impact of these parameters on HER. ${ }^{117-121}$ Figure 5 provides an overview of some of the most significant results.

Starting with nickel phosphide, multifaceted and hollow $\mathrm{Ni}_{2} \mathrm{P}$ NCs were examined (Figure 5A). While still higher than Pt, a competitive overpotential of $-116 \mathrm{mV}$ was measured at -10 $\mathrm{mA} / \mathrm{cm}^{2}$ (Figure 5D). ${ }^{118}$ As the exposed facets can play an important role in catalysis, $\mathrm{Ni}_{2} \mathrm{P}$ nanospheres (NSs), which predominantly expose (001) facets on their surface, were compared with $\mathrm{Ni}_{2} \mathrm{P}$ nanorods (NRs), which possess a high density of exposed (210) surfaces. ${ }^{118,122}$ The latter exhibited a larger overpotential $(-270 \mathrm{mV})$ and around 10 times lower turnover frequency when tested under the same conditions. Similar findings were reported by Zhou et al. ${ }^{123}$ They synthesized small $\mathrm{Ni}_{2} \mathrm{P}$ NCs ranging from $2 \mathrm{~nm}$ to $10 \mathrm{~nm}$ with large accessible surface areas and predominant (001) surfaces. Among these catalysts, $5.4 \mathrm{~nm} \mathrm{Ni}_{2} \mathrm{P}$ NCs showed superior HER activity with a reduced Tafel slope and overpotential of $-93 \mathrm{mV}$ at $-20 \mathrm{~mA} \mathrm{~cm}{ }^{-2}$. Such results testify to the importance of tuning both shape and size to achieve optimal results.

When investigating cobalt phosphide, multifaceted and hollow $\mathrm{CoP}$ and $\mathrm{Co}_{2} \mathrm{P}$ NCs with equivalent morphologies were synthesized and their HER activities were compared in order to assess compositional effects (Figure 5B). ${ }^{117} \mathrm{CoP}$ was found to be more active than pure Co 
and $\mathrm{Co}_{2} \mathrm{P}$ with an overpotential of $-85 \mathrm{mV}$ at $-20 \mathrm{~mA} / \mathrm{cm}^{2}$ (Figure 5E). ${ }^{124}$ For this system, interestingly, the comparison of CoP NCs with highly branched CoP NRs with predominant (111) facets revealed that the performance of CoP toward the HER is intrinsic to the composition and that the shape is not critical. ${ }^{125}$ Following up with the same colloidally synthesized CoP NCs, Ha et al. performed a comprehensive study by employing DFT along with experiments to investigate the stability and active sites during HER. ${ }^{126}$ The authors showed that the HER activity decreases with potentiostatic holding at potentials above 0.4 $\mathrm{V}_{\mathrm{RHE}}$, accompanied by the leaching of $\mathrm{P}$ and a decreased ratio of $\mathrm{Co} / \mathrm{P}$, which was supported by energy-dispersive X-ray (EDX) spectroscopy results. X-ray absorption spectroscopy (XAS) demonstrated the change of the signals for Co and $\mathrm{P}$, suggesting the concomitant formation of (oxy)phosphate as the activity reduces. DFT calculations identified the surface P as the HER active site in the case of $\mathrm{CoP}$, which contradicted some previous studies that indicated the active site to be the metal. ${ }^{116}$

Because of the Earth abundance and low cost of iron, FeP represents another promising phosphide for the HER. Colloidally synthesized FeP NCs of similar size and morphology to the CoP NCs (Figure 5C) were shown to outperform the latter, with an overpotential of -50 $\mathrm{mV}$ in acidic conditions and $-102 \mathrm{mV}$ in neutral electrolyte required to reach a current density of $10 \mathrm{~mA} / \mathrm{cm}^{2}$ (Figure 5F). ${ }^{119}$ The main challenge associated with FeP NCs remains to be the long-term stability, which is poorer than for $\mathrm{Ni}_{2} \mathrm{P}$ and $\mathrm{CoP} \mathrm{NCs}$.

In addition to crystalline phosphides, colloidally synthesized amorphous WP and MoP materials have also been reported as interesting electrocatalysts for the HER in acidic solutions. ${ }^{120,121} 3.1 \mathrm{~nm}$ WP NCs were found to require overpotentials of $-120 \mathrm{mV}$ and -140 $\mathrm{mV}$ to produce operationally relevant current densities of -10 and $-20 \mathrm{~mA} / \mathrm{cm}^{2}$ while $4.2 \mathrm{~nm}$ MoP NCs needed even lower overpotentials $(-90 \mathrm{mV}$ and $-105 \mathrm{mV})$ to generate the same current densities, outperforming other MoP nanostructured electrocatalysts. ${ }^{18}$ These data suggest that Mo might be more promising than Co and that the small particles attainable by colloidal chemistry might offer specific surface structuring which increases their intrinsic activity. 

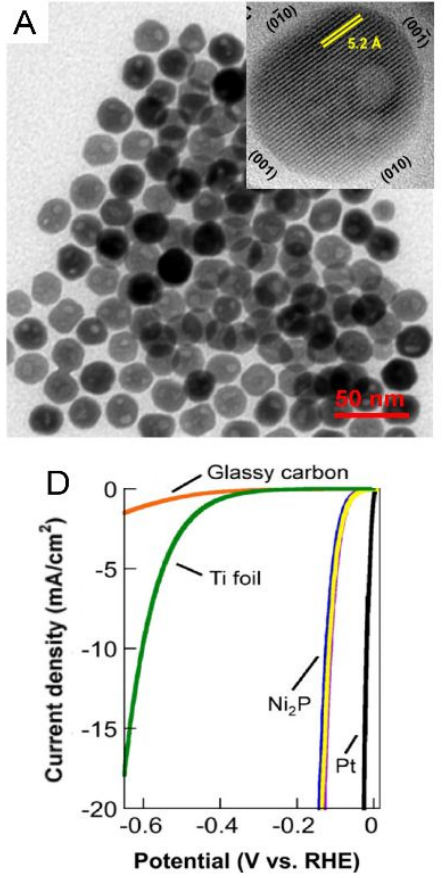
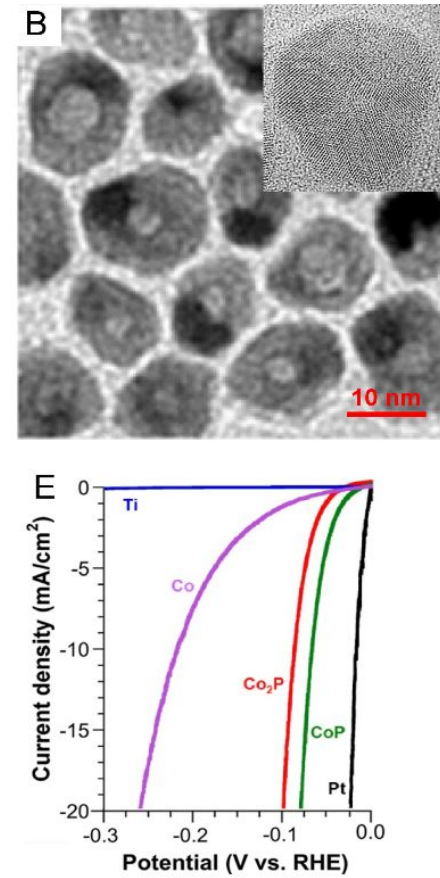
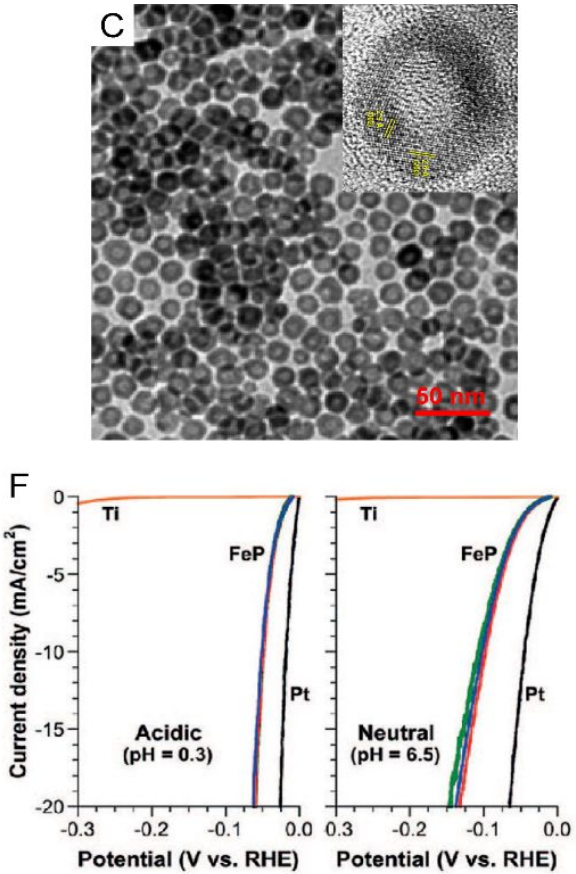

Figure 5. Metal phosphide NCs for HER. (A-C) Transmission electron microscopy (TEM) images of $\mathrm{Ni}_{2} \mathrm{P}, \mathrm{CoP}$ NCs and FeP NCs, respectively. (D-F) Linear-sweep voltammograms for (D) $\mathrm{Ni}_{2} \mathrm{P}$ in $0.5 \mathrm{M} \mathrm{H}_{2} \mathrm{SO}_{4}$, (E) $\mathrm{Co}, \mathrm{CoP}$, and $\mathrm{Co}_{2} \mathrm{P} \mathrm{NCs}$ in $0.5 \mathrm{M} \mathrm{H}_{2} \mathrm{SO}_{4}$ and (F) FeP NCs in acidic solution ( $\left.0.5 \mathrm{M} \mathrm{H}_{2} \mathrm{SO}_{4}\right)$ and neutral solution (1.0 $\mathrm{M}$ phosphate buffered saline). All the NCs were deposited on $\mathrm{Ti}$ foil substrates and native ligands were removed by annealing at $450{ }^{\circ} \mathrm{C}$ in $\mathrm{H}_{2}(5 \%) / \mathrm{N}_{2}(95 \%)$. (A,D) Adapted with permission from reference 118. Copyright 2013, American Chemical Society. (B,E) Adapted with permission from reference 117. Copyright 2015, American Chemical Society. (C,F) Adapted with permission from reference 119. Copyright 2014, American Chemical Society.

\subsection{Metal chalcogenides}

Turning to the transition metal chalcogenides, this class of materials has received attention from the colloidal NC community only more recently. While still few, the reports on heterostructures reveal the possibility to use them as pre-catalysts for materials with lower PMG content and performance similar or improved compared to commercial catalysts under alkaline conditions. ${ }^{127-129}$ As commented above, the colloidal synthesis of molybdenum sulphides is still a challenge, therefore while these chalcogenides have emerged as a great alternative to $\mathrm{Pt}$, not much has been done with colloidal methods. ${ }^{130,131}$

Interesting studies have been reported by L. Manna and co-workers and they have dedicated particular attention to the catalyst transformation during operation, something which is enabled by the sample uniformity attainable by colloidal chemistry. As the first example, they 
synthesized CoSe NCs decorated with Ru clusters with the aim of improving the stability of PGMs under operational conditions. ${ }^{132}$ They showed that the Ru-CoSe system undergoes chemical and structural transformations during the HER; indeed, CoSe NCs with an initial hexagonal structure converted into $\mathrm{CoSe}_{2} \mathrm{NCs}$ with a cubic structure. Co that leached from the catalyst formed a Co oxide/hydroxide layer, which was proposed to help the $\mathrm{H}_{2} \mathrm{O}$ adsorption and dissociation under high local $\mathrm{pH}$ environment (Figure 6B). In addition, the presence of $\mathrm{Ru}$ enhanced the electrocatalytic properties of CoSe NCs by improving the HER kinetics and reducing the charge-transfer resistance. All of these phenomena were suggested to trigger the increased HER activity of the final $\mathrm{Ru}-\mathrm{CoO}_{\mathrm{x}} / \mathrm{Co}(\mathrm{OH})_{\mathrm{x}}-\mathrm{CoSe}_{2}$ nanocomposite after $30 \mathrm{~h}$ of operation, which was comparable to or even higher than that of $\mathrm{Pt} / \mathrm{C}$ at high overpotentials (Figure 6A,C).

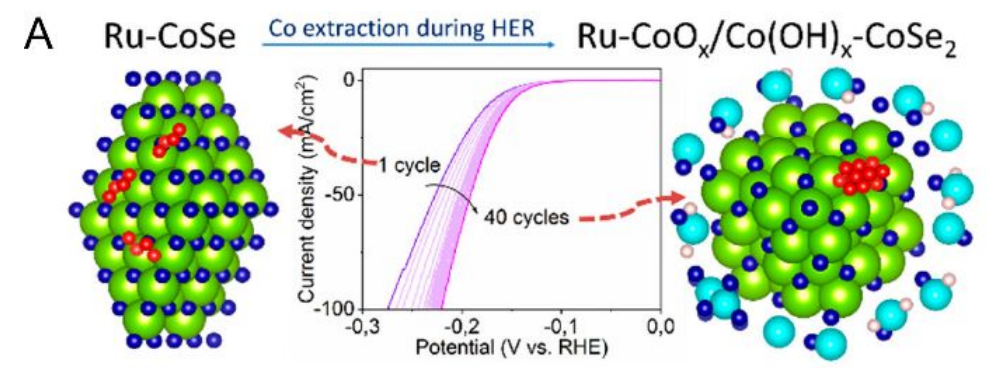

B
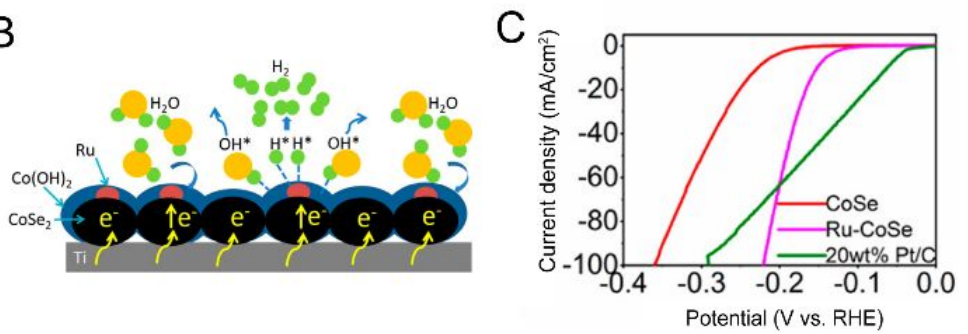

Figure 6. Metal chalcogenide NCs for HER. (A) Linear-sweep voltammograms from the first to the $40^{\text {th }}$ cycle along with the demonstration of structural transformation in Ru-CoSe during these cycles. (B) Schematic illustration of the HER mechanism on the Ru$\mathrm{CoO}_{\mathrm{x}} / \mathrm{Co}(\mathrm{OH})_{\mathrm{x}}-\mathrm{CoSe}_{2}$ electrocatalyst. (C) Linear-sweep voltammograms for Co-Se, Ru-CoSe and Pt/C NCs. Adapted with permission from reference 132. Copyright 2019, American Chemical Society.

In another recent study, Najafi et al. have synthesized CdSe octapods (OCPs) by a colloidal seeded-growth approach and decorated their surface with Pt NCs. ${ }^{127}$ The hierarchical Pt@CdSe-OCPs were assembled onto carbon-nanotube (CNT) bucky-paper and revealed a significant increase of the mass-normalized HER activity compared to that of $\mathrm{Pt} / \mathrm{C}$ (13.4 times 
higher in $0.5 \mathrm{M} \mathrm{H}_{2} \mathrm{SO}_{4}$ and 21 times higher in $1 \mathrm{M} \mathrm{KOH}$, at $-0.15 \mathrm{~V}_{\mathrm{RHE}}$ ). Moreover, the catalyst exhibited long-term stability $(>20 \mathrm{~h}$ ) in both of the electrolytes investigated, while the $\mathrm{Pt} / \mathrm{C}$ reference catalyst progressively degraded under alkaline conditions. The improved catalytic performance were attributed to the optimal surface-to-bulk atomic ratio of $\mathrm{Pt}$ and the chemical interaction between Pt and the CdSe surface that prevents Pt dissolution/deactivation effects, often observed with low Pt mass loadings.

In another example within the same class of materials, PtNi/NiS nanowires (NWs) with different molar ratios of $\mathrm{Pt}$ to $\mathrm{Ni}$ (sulfurated in oleylamine) were shown to cooperate synergistically towards HER in alkaline conditions. ${ }^{128} \mathrm{Pt}_{3} \mathrm{Ni}_{2} \mathrm{NWs}-\mathrm{S} / \mathrm{C}$ delivered a maximum current density of $37.2 \mathrm{~mA} / \mathrm{cm}^{-2}$ at an overpotential of $70 \mathrm{mV}$, which is around 10 times higher than that of commercial Pt/C. Based on DFT calculations, the energy barrier for breaking the HO-H bond in water on NiS (100) surface is reduced compared to Pt (111). Moreover, the $\mathrm{H}$ binding energy on the $\mathrm{Pt}_{3} \mathrm{Ni}_{2}$ (111) surface is closer to the optimal value, revealing the $\mathrm{Pt}_{3} \mathrm{Ni}_{2}$ NWs-S/C as a superior HER electrocatalyst.

\section{OER/ORR}

The oxygen reduction (ORR) and oxygen evolution (OER) reactions are two key reactions relevant in various energy-related applications. ${ }^{133-135}$ Rechargeable metal-air batteries, fuel cells and electrolyzers, all rely on the oxygen electrocatalysis, which still suffers from sluggish kinetics, making the search of effective Earth-abundant and reasonably priced catalysts crucial. ${ }^{136-139}$ To date, the best known catalysts in acidic conditions is Pt for ORR (Figure 7) and ruthenium and iridium oxides for OER. On the other hand, in alkaline environment oxides are used for both reactions, i.e. manganese oxide for ORR and a combination of nickel-ironcobalt oxides for OER. In both cases, an overpotential of around $0.3-0.4 \mathrm{~V}$ at $10 \mathrm{~mA} / \mathrm{cm}^{2}$ is still needed. ${ }^{133-135}$ Finding valid alternative to noble metal containing catalysts is one of the main challenges in acidic media for both OER and ORR. ${ }^{140,141}$ Improving the reaction kinetics of the oxides is instead the main issue in alkaline media. ${ }^{140,141}$ Enhancing the catalyst stability is also crucial in any environment.

Compared to metals, oxide NCs often suffer from a poorer control on size and shape because their synthesis is still challenging, especially when it comes to multication oxides. Studies on iridium oxide NCs for OER have been conducted, yet the advantage of using colloidal methods over other techniques is not immediately clear as the size monodispersity and shape uniformity 
in these samples was often poor. ${ }^{142-146}$ Very limited examples have been reported for ORR in alkaline conditions, with work on $\mathrm{M}(\mathrm{II})$-substituted magnetite $\mathrm{M}_{\mathrm{x}} \mathrm{Fe}_{3}-\mathrm{xO}_{4}\left(\mathrm{M}_{\mathrm{x}} \mathrm{Fe}_{1}-\mathrm{xO} \cdot \mathrm{Fe}_{2} \mathrm{O}_{3}\right)$ $(\mathrm{M}=\mathrm{Mn}, \mathrm{Fe}, \mathrm{Co}, \mathrm{Cu}) \mathrm{NCs}$ as promising substitutes of Pt being one of these. ${ }^{147}$ Significant studies have been performed on non-stoichiometric mixed metal oxides, however here colloidal chemistry could provide only the precursor to form the final catalysts upon annealing above $800{ }^{\circ} \mathrm{C} .{ }^{148,149}$ While being a powerful and promising approach to obtain shaped-controlled mixed metal oxides, these catalysts were not directly obtained via colloidal chemistry and were finally in the form of powders, therefore are not included in this review. ${ }^{148,149}$

Overall, most of the research efforts which highlight the advantage of using well-defined colloidal NCs have been directed towards metal-based ORR catalysts in acidic conditions.

Below, we focus on three classes of materials which have been more widely investigated and are therefore representative of the contribution of nanochemistry to the field up to now. Details of this contribution are discussed below for each class of catalysts. A summary of all data is reported in Table $\mathbf{S 2}$.

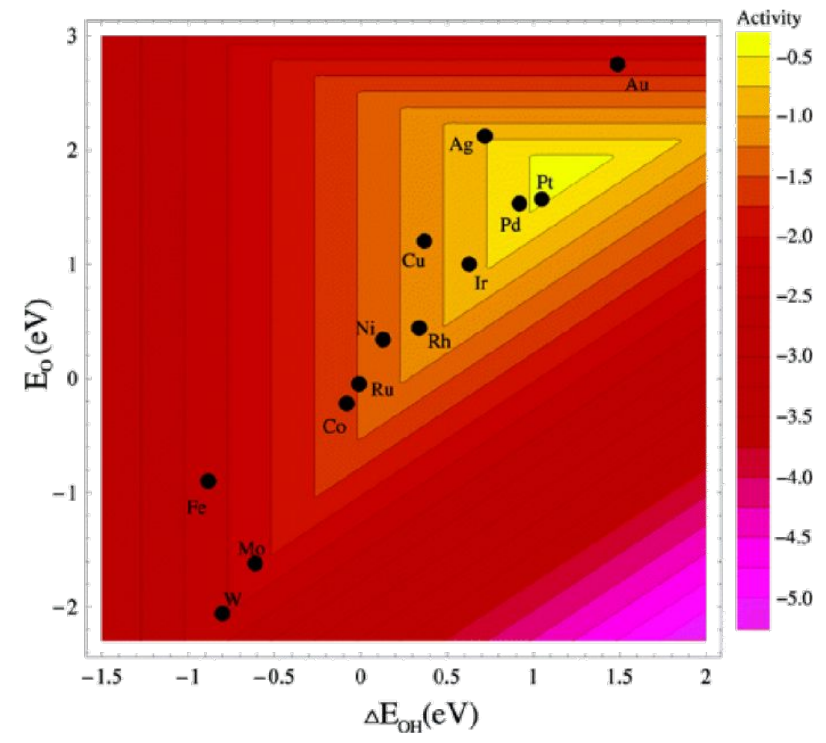

Figure 7. Volcano plot reporting trends in ORR activity as a function of the $\mathrm{O}$ and the $\mathrm{OH}$ binding energies as descriptors. Reproduced with permission from reference 150. Copyright 2004, American Chemical Society.

\subsection{Size- and shape-controlled Pt and Pd NCs}

$\mathrm{Pt}$ is the most active monometallic catalysts for ORR in acidic media (Figure 7). ${ }^{150}$ Theory and experiments have evidenced a structure-dependent selectivity. In particular, it was reported that the activity decreases as $\mathrm{Pt}(110)>\mathrm{Pt}(111)>\mathrm{Pt}(100)$ in a nonadsorbing electrolyte (e.g. $\left.\mathrm{HClO}_{4}\right)$, 
and as $\operatorname{Pt}(110)>\operatorname{Pt}(100)>\operatorname{Pt}(111)$ in an adsorbing electrolyte $\left(\right.$ e.g. $\left.\mathrm{H}_{2} \mathrm{SO}_{4}\right) \cdot{ }^{151-153}$ Hence, during the last decade, Pt NCs with a variety of sizes and shapes were synthesized and tested towards ORR under similar conditions (Table S2) in the quest of translating the facetdependent activity of single crystals to "real world" catalysts. ${ }^{38,154-158}$ For example, Wang et al. have compared monodispersed Pt NCs with sizes tunable from $3 \mathrm{~nm}$ to $7 \mathrm{~nm}$ and controlled polyhedral, truncated cubic and regular cubic shapes. ${ }^{159,160}$ They demonstrated that $7 \mathrm{~nm} \mathrm{Pt}$ cubes, enclosed in (100) facets, were significantly more active than $3 \mathrm{~nm}$ polyhedral and $5 \mathrm{~nm}$ truncated cubic NCs, both exposing (100) and (111) facets, when tested in $\mathrm{H}_{2} \mathrm{SO}_{4}$ as the electrolyte, consistently with the single crystal studies (Figure 8A-D). Here, shape effects were found to dominate over size-effects. Furthermore, the cubes were twice more active compared to a commercial Pt catalyst tested in the same RDE setup. Later on, with more complex shapes, even higher activities were obtained. ${ }^{80,161,162}$ Zhang et al. have synthesized Pt cubic and octahedral nanocages enclosed by (100) and (111) facets, respectively, by depositing a few atomic layers of $\mathrm{Pt}$ on $\mathrm{Pd} \mathrm{NCs}$ with well-defined shapes and then etching away the $\mathrm{Pd}$ templates. ${ }^{80}$ The mass activity of the octahedral nanocages was found to be almost twice that of the cubic nanocages and 8 times the one of commercial $\mathrm{Pt} / \mathrm{C}(0.75 \mathrm{~A} / \mathrm{mg}$ vs $0.4 \mathrm{~A} / \mathrm{mg}$ vs $0.14 \mathrm{~A} / \mathrm{mg}$ at $\left.0.9 \mathrm{~V}_{\mathrm{RHE}}\right)$. ). As octahedral nanocages are rich in (111) planes, the improved catalytic performance is attributed to the higher activity of $\operatorname{Pt}(111)$ than $\operatorname{Pt}(100)$ toward ORR in $0.1 \mathrm{M} \mathrm{HClO}_{4}$. Referring to the same point of the high $\mathrm{Pt}(111)$ activity, He et al. and Wang et al., reported about Pt icosahedral nanocages (Figure 8E,F) and demonstrated up to $\sim 10$ times and 1.5 times increase in specific and mass activity over commercial $\mathrm{Pt} / \mathrm{C}$ catalysts and octahedral nanocages, respectively (reaching $3.50 \mathrm{~mA} / \mathrm{cm}^{2}$ and $1.28 \mathrm{~A} / \mathrm{mg}$ at $0.9 \mathrm{~V}_{\mathrm{RHE}}$ ). ${ }^{161,162}$ The comparison between the activity and stability of different facets was further expanded by Li et al. who investigated the morphological evolution of Pt cubes, octahedra and cuboctahedra by potential-induced Pt dissolution/re-deposition when cycling between 0.05 and $1.3 \mathrm{~V}_{\mathrm{RHE}}$, which was chosen as an accelerated catalyst degradation protocol. ${ }^{163}$ In addition to assessing that smaller particles are less stable than bigger particles, the authors highlighted the role of the density of the low-coordinated atoms in the relationship between stability and morphology changes. Based on scanning tunneling microscopy (STM) investigations on $\operatorname{Pt}(111)$ and $\operatorname{Pt}(100)$, they proposed that the low-coordinated atoms along the step-edges are much less stable than those on the facets, which might indicate that they are also more active. Highresolution transmission electron microscopy (HRTEM) analysis of the NCs before and after ORR suggested that the NC shapes are metastable, which leads to the observed change into thermodynamically equilibrated size and shape and, finally, to a similar activity towards ORR. 
$7 \mathrm{~nm}$ cuboctahedral Pt NCs were finally recommended as the trade-off between initial mass activity and durability performance.
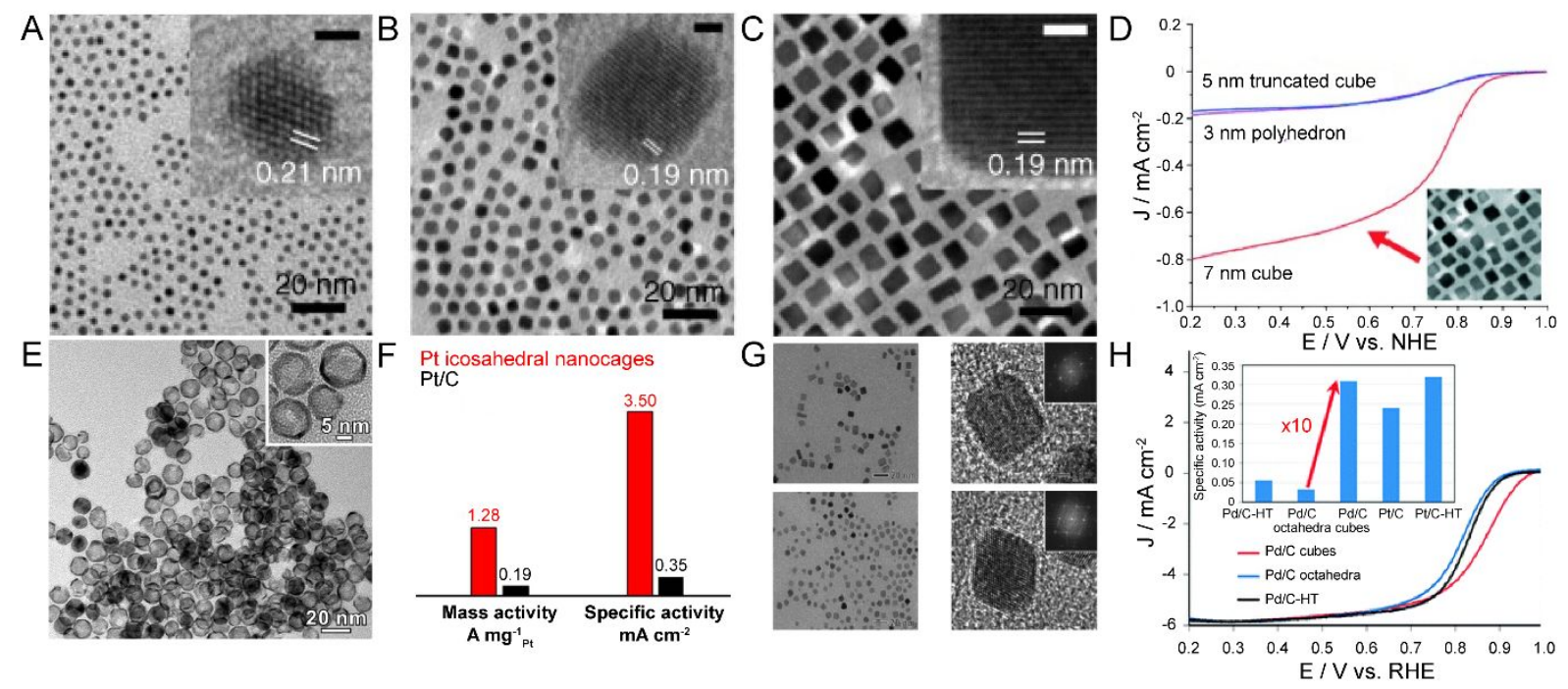

Figure 8. Size- and shape- controlled Pt and Pd NCs for ORR. Representative TEM images of (A) $3 \mathrm{~nm}$ polyhedral, (B) $5 \mathrm{~nm}$ truncated cubic and (C) $7 \mathrm{~nm}$ cubic Pt NCs. The insets are the representative HRTEM images of corresponding single particles, showing $\operatorname{Pt}(111), \operatorname{Pt}(100)$, and $\operatorname{Pt}(100)$ lattice fringes, respectively (scale bars correspond to $1 \mathrm{~nm}$ ). (D) Corresponding anodic polarization curves in oxygen saturated $0.5 \mathrm{M} \mathrm{H}_{2} \mathrm{SO}_{4}$ measured in an RDE set-up. Adapted with permission from reference 159. Copyright 2008, Wiley. (E) TEM image of the Pt icosahedral nanocages and (F) comparison of the mass and specific activity for the carbonsupported Pt icosahedral nanocages and a commercial Pt/C catalyst obtained at $0.9 \mathrm{~V}_{\mathrm{RHE}}$ in 0.1 $\mathrm{M} \mathrm{HClO}_{4}$. Adapted with permission from reference 162. Copyright 2016, American Chemical Society. (G) TEM (left) and HRTEM images (right) of Pd cubes (top) and octahedral (bottom), respectively. $(\mathrm{H})$ Anodic polarization curves for carbon-supported commercial $\mathrm{Pd} / \mathrm{C}$ and $\mathrm{Pd} / \mathrm{C}$ octahedra and cubes in $0.1 \mathrm{M} \mathrm{HClO}_{4}$ in an RDE set-up. The inset shows the comparison of the specific activities at $0.9 \mathrm{~V}_{\mathrm{RHE}}$. Adapted with permission from reference 164. Copyright 2011, Royal Society of Chemistry.

According to the scaling relations (Figure 7), Pd is the metal next to Pt in terms of its intrinsic activity towards ORR. However, it has been generally less explored both in bulk and in colloidal NC form because of the lower stability. ${ }^{165}$ A facet dependent activity has been reported also for $\mathrm{Pd}$, which increase as $\mathrm{Pd}(110)<\mathrm{Pd}(111)<<\mathrm{Pd}(100)$ in $\mathrm{HClO}_{4}$ electrolyte. ${ }^{164,166}$ Therefore, similarly to $\mathrm{Pt}$, the activity dependence on the size and shape of 
Pd NCs has been studied. ${ }^{166,167}$ For example, Shao et al. have synthesized $10 \mathrm{~nm}$ cubes and octahedra and found that the specific activity experienced a 10 times increase from octahedra with predominant (111) surfaces to cubes with mainly (100) surfaces, thus in agreement with the trends predicting that (100) facets are more active, and reaching activity values comparable with those of commercial Pt catalysts. (Figure 8G and H). ${ }^{164}$

To summarize this section, Table S2 evidences that Pt octahedral nanocages exposing Pt (111) surfaces are the most active compared to other catalysts tested under very similar conditions in $0.1 \mathrm{M} \mathrm{HClO}_{4}$, which is in line with the trends from single crystal studies. In addition to the exposed facets, the shape control achievable by colloidal chemistry evidences that a hollow structure is also beneficial, as it facilitates the $\mathrm{O}_{2}$ accessibility to the active sites.

\subsection{NC alloys of Pt and early transition metals as ORR catalysts}

In the quest of reducing the Pt content in the ORR catalyst while also improving the activity compared to the commercial $\mathrm{Pt} / \mathrm{C}$, alloying $\mathrm{Pt}$ with early transition metals including $\mathrm{Fe}, \mathrm{Co}, \mathrm{Ni}$ is a promising strategy. ${ }^{168}$ Among these, Pt-Ni alloyed NCs have been the most explored catalysts so far leading to quite impressive breakthroughs. Stamenkovic et al. initiated such effort with DFT calculations and single crystal surface studies under ultra-high vacuum (UHV) evidencing that the $\mathrm{Pt}_{3} \mathrm{Ni}(111)$ surface is 10 times more active than the corresponding $\mathrm{Pt}(111)$ surface and 90 times more active than $\mathrm{Pt} / \mathrm{C}$ catalysts (Figure 9A). ${ }^{153}$ This finding was attributed to a combination of electronic effects ( $d$-band center position) and geometric effects (i.e. namely Pt-rich surface). 

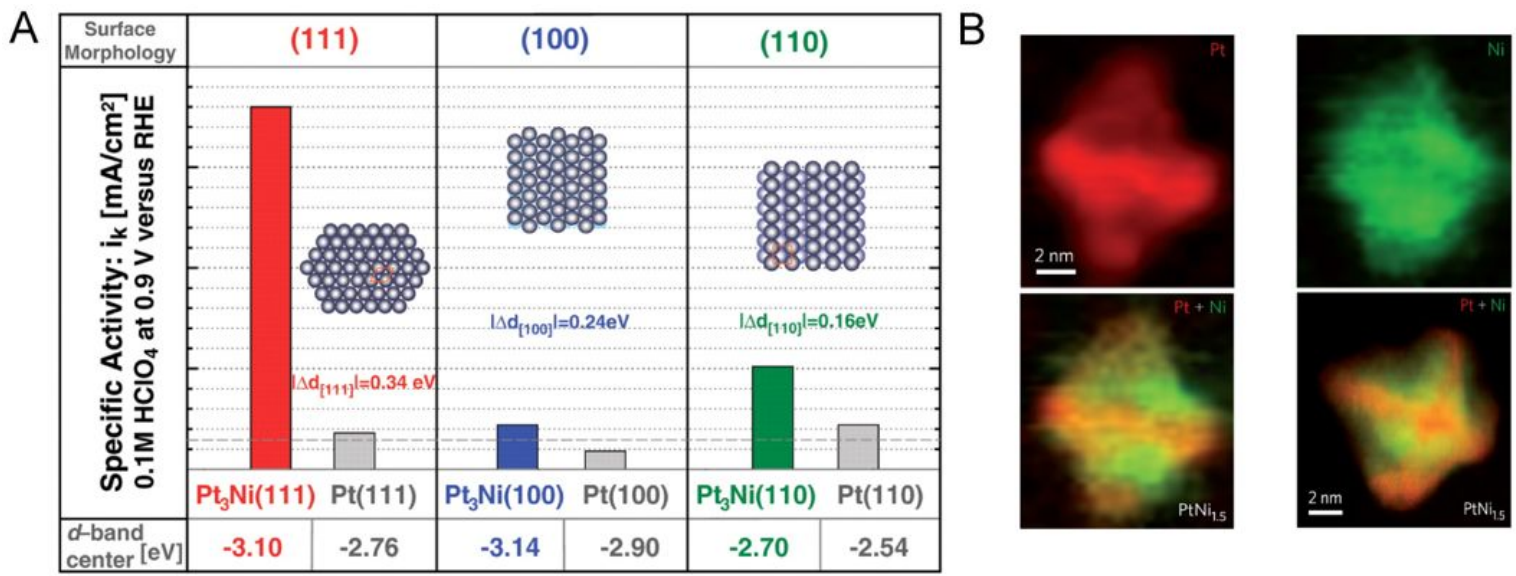

C
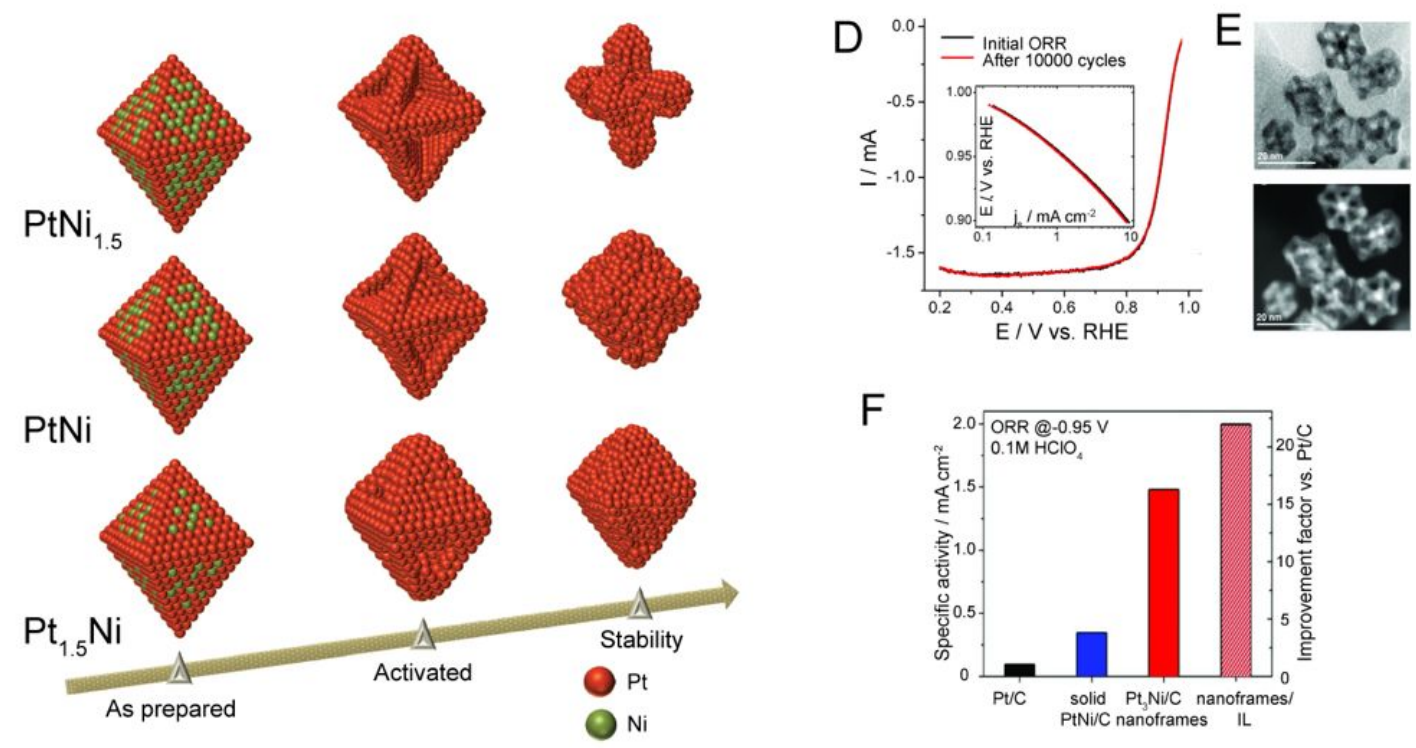

$\mathrm{F}$

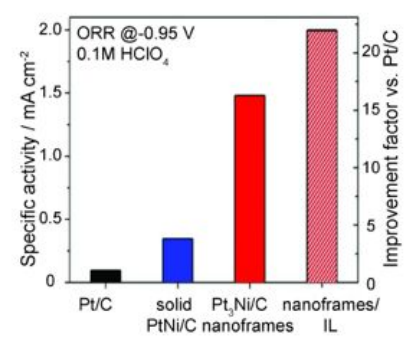

Figure 9. Pt-Ni alloyed NCs for ORR. (A) Influence of the surface morphology and electronic properties on the ORR performance. Rotating ring-disk electrode (RRDE) measurements for ORR in $0.1 \mathrm{M} \mathrm{HClO}_{4}$ on $\mathrm{Pt}_{3} \mathrm{Ni}(h k l)$ surfaces as compared to the corresponding $\mathrm{Pt}(h k l)$ surfaces (a horizontal dashed gray line marks specific activity of polycrystalline Pt) are shown. Specific activity is given as a kinetic current density $i_{k}$, measured at $0.9 \mathrm{~V}_{\mathrm{RHE}}$. Values of d-band center position obtained from ultra-violet photoemission spectroscopy (UPS) are listed for each surface morphology and compared between corresponding $\mathrm{Pt}_{3} \mathrm{Ni}(h k l)$ and $\mathrm{Pt}(h k l)$ surfaces. Adapted with permission from reference 153. Copyright 2007, AAAS. (B) Electron energy loss spectroscopy (EELS) element maps of $\mathrm{PtNi}_{1.5}$ showing the segregated distribution of $\mathrm{Pt}$ (red) and $\mathrm{Ni}$ (green) in a particle close to the $\langle 110\rangle$ zone axis (top and bottom left). Composite image of a high-angle annular dark-field (HAADF) image showing mainly Pt (red) and an EELS map showing Ni (green) in a particle close to the $\langle 100\rangle$ zone axis (bottom right). (C) Schematic representation of the $\mathrm{Pt}_{\mathrm{x}} \mathrm{Ni}_{1-\mathrm{x}} \mathrm{NCs}$ morphology and surface structure changes after electrochemical surface activation ( 25 cycles, $0.06-1.0 \mathrm{~V}_{\mathrm{RHE}}$ potential range) and stability 
(4000 cycles, $0.6-1.0 \mathrm{~V}_{\mathrm{RHE}}$ potential range) tests relative to as-synthesized NCs in $0.1 \mathrm{M}$ $\mathrm{HClO}_{4}$ in a RDE set-up. Adapted with permission from reference 169. Copyright 2013, Nature. (D) ORR polarization curves and (inset) corresponding Tafel plots of $\mathrm{Pt}_{3} \mathrm{Ni}$ nanoframes before and after 10,000 cycles in $0.1 \mathrm{M} \mathrm{HClO}_{4}$ and $0.6-1.0 \mathrm{~V}_{\mathrm{RHE}}$ potential range (E) Bright-field (top) and dark-field (bottom) scanning transmission electron microscopy (STEM) images of carbon-supported $\mathrm{Pt}_{3} \mathrm{Ni}$ nanoframes/C after cycling. (F) Specific ORR activity of $\mathrm{Pt}_{3} \mathrm{Ni}$ nanoframes compared to other state-of-the-art catalysts (IL - ionic liquid) at $-0.95 \mathrm{~V}_{\mathrm{RHE}}$ in 0.1 $\mathrm{M} \mathrm{HClO}_{4}$. Adapted with permission from reference 170. Copyright 2014, AAAS.

In the following decade, these findings inspired a series of studies aiming at the synthesis of $\mathrm{PtNi}_{3} \mathrm{NCs}$ exposing (111) facets on their surface. To start with, Choi et al. synthesized highly active $9 \mathrm{~nm} \mathrm{Pt}_{2.5} \mathrm{Ni}$ octahedra and demonstrated that they can be produced in a scalable way with the use of a continuous-flow droplet reactor. ${ }^{171,172}$ These catalysts reached an outstanding 51-fold increase in ORR activity compared to $\mathrm{Pt} / \mathrm{C}$ catalysts allowed by the predominant exposure of $\mathrm{Pt}_{3} \mathrm{Ni}(111)$ surfaces. $\mathrm{Wu}$ et al. synthesized truncated $\mathrm{Pt}_{3} \mathrm{Ni}$ octahedra and demonstrated that the activity strongly depends on the (111) surface fraction. ${ }^{173} \mathrm{Ni}-\mathrm{Pt}$ alloyed NCs with different shapes and composition provided further insights into this system. ${ }^{174-177}$ For example, $\mathrm{Pt}_{3} \mathrm{Ni}$ icosahedra were shown to possess an additional $50 \%$ increase in specific activity compared to octahedra. ${ }^{174-176}$ While both crystals expose (111) surfaces, DFT and molecular dynamics simulations indicated that this improvement may arise from strain-induced electronic effects. ${ }^{176}$ Urchin-like $\mathrm{PtNi}, \mathrm{Pt}_{2} \mathrm{Ni}$ and $\mathrm{Pt}_{3} \mathrm{Ni}$ were synthesized and their performance compared by Choi et al. Here, the $\mathrm{Pt}_{2} \mathrm{Ni}$ catalysts were demonstrated to have a similar performance as $\mathrm{Pt}_{3} \mathrm{Ni}$ reaching 20 times increase in specific activity compared to the $\mathrm{Pt}$ catalyst as a result of combining structural factors and compositional effects. The authors note that, the urchin-like structures are dominated by (100) rather than (111) facets. Better performance compared to commercial Pt NCs were attributed to the alloying effect, together with a low specific anion adsorption, which is desired for implementation into commercial hydrogen fuel cells because of possible surface poisoning by sulfonic groups of Nafion. This is not the case for the (111) surface, which is more susceptible to poisoning via specific anion adsorption. ${ }^{178}$ An important set of follow-up studies focused on the morphological and compositional evolution of the different shape-controlled Pt-Ni catalysts during ORR. Cui et al. studied $\mathrm{Pt}_{1.5} \mathrm{Ni}, \mathrm{PtNi}, \mathrm{PtNi}_{1.5}$ octahedral NCs. ${ }^{169}$ Via carefully executed electron microscopy studies, they evidenced that the two elements are phase segregated in the as-prepared catalysts and that $\mathrm{Ni}$ leaches out of the structure during the activation and reaction (Figure 9B and $\mathbf{C}$ ). This 
dealloying process results in the generation of concave octahedral NCs, and the ORR activity is ultimately determined by a balance between the thickness of the pure Pt shell, the subsurface Ni content and the ratio of the remaining (111) facets.

Later on, Huang et al. demonstrated that $\mathrm{Ni}$ leaching can be prevented by doping the $\mathrm{Pt}_{3} \mathrm{Ni}$ octahedra with molybdenum. ${ }^{179}$ The Mo-doped catalysts showed 81-fold and 73-fold enhancement in specific and mass activity compared to the commercial $\mathrm{Pt} / \mathrm{C}$ catalyst and 4fold enhancement in specific activity compared to the undoped counterpart. Even more importantly, a stable operation over 8000 cycles was observed. Based on DFT calculations, the improved performance was attributed to the formation of surface Mo-oxide species, which stay near the particle edges and may capture surface $\mathrm{Ni}$, preventing its leaching.

As an alternative, the dealloying processes and element-specific segregation in the Pt-Ni catalysts have been exploited to construct more active catalysts. For example, Niu et al. reported about the formation process of nanoframe catalysts from Pt-Ni rhombic dodecahedra which relies on spontaneous corrosion upon air exposure without any harsh treatment with chemicals or application of potential involved. ${ }^{45}$ The outstanding performance was ascribed to the effect of morphology on the coordination and strain of surface atoms, and to the overall three-dimensional molecular accessibility.

Chen et al. have demonstrated that, once formed, the $\mathrm{Pt}_{3} \mathrm{Ni}$ nanoframes possess remarkable stability under operation (the potential between 0.6 and $1.0 \mathrm{~V}$ for a duration of 10,000 potential cycles at different sweep rates from 2 to $200 \mathrm{mV} \mathrm{s}^{-1}$ ) (Figure9D and E). Furthermore, they show a 22-fold and 36-fold increase in specific and mass activity, respectively, compared to state-of-the-art Pt/C catalysts, when infilled with protic ionic liquids that increase the $\mathrm{O}_{2}$ concentration close to the catalyst surface (Figure 9F). ${ }^{170,180}$

Follow-up studies from the same group have then provided further insights into these nanoframe catalysts. Becknell et al. focused on the compositional tunability. ${ }^{181}$ By playing with the $\mathrm{Pt} / \mathrm{Ni}$ ratio they obtained new architectures, referred to as excavated nanoframes (E-NF) instead of the hollow nanoframes (H-NF). The E-NFs exhibited Pt-rich sheets extending inward from Pt-rich edges, whereas the H-NFs had empty space inside Pt-rich edges, but higher Nicontent in the near edge region. These differences resulted in remarkable improvement in the ORR intrinsic activity for the E-NFs. The E-NFs possessed an increased intrinsic activity compared to H-NFs, which was attributed to their extended surfaces that were more enriched in $\mathrm{Ni}^{181}$ In a very systematic study, Chen et al. compared different corrosion procedures for the preparation of the nanoframes: the original ambient oxidation, a corrosion process involving acetic acid and a corrosion process involving nitric acid. ${ }^{182}$ Eventually, stable 
operations over 30,000 cycles were observed when using the oxidative nitric acid which leads to a lower Ni content and a less-defective surface. Instead, the ambient oxidation leads to the highest initial activity but also the fastest performance decay because the surface reconstructive process and the Ni leaching are continuing during catalysis. Overall, these results highlight that a higher Ni content promotes activity but also instability, and thus a trade-off must be reached.

Pt-Co NCs come after Ni-Pt in terms of dedicated research effort, which is lower probably because the overall stability remains an issue. Lee et al. synthesized monodispersed bimetallic NCs with a series of compositions $\left(\mathrm{Pt}_{3} \mathrm{Co}, \mathrm{PtCo}, \mathrm{PtCo}_{2}\right.$ and $\left.\mathrm{PtCo}_{3}\right)$ as structurally ordered $\mathrm{L}_{0}$ intermetallics. ${ }^{183}$ The authors were able to correlate the composition to the prominence of the $\mathrm{L} 1_{0}$ phase, which was then found to impact the ORR performance (higher Co content, higher order, higher activity). All catalysts outperformed the commercial $\mathrm{Pt} / \mathrm{C}$ in terms of activity, which demonstrate the beneficial effects of alloying. While $\mathrm{PtCo}_{3}$ showed the highest activity, it was also the least stable due to Co leaching. PtCo was shown to be the best compromise between activity and stability. Wang et al. have then demonstrated that a thin shell of Pt around a structurally ordered $\mathrm{PtCo}_{3}$ intermetallic core contributes both to enhanced performance and durability. ${ }^{184}$ Supported on carbon, these catalysts were compositionally and structurally stable up to 5,000 potential cycles. ${ }^{184,185}$ Indeed, the Pt skin avoids leaching of the transition metals into the acidic solution. ${ }^{186} \mathrm{Pt}-\mathrm{Co}$ nanoframes were also synthesized from rhombic dodecahedra in a similar manner to what was done for Pt-Ni and studied for ORR. ${ }^{187}$ The $\mathrm{Pt}_{82} \mathrm{Co}_{18}$ nanoframes exhibited higher mass activity than commercial $\mathrm{Pt} / \mathrm{C}$ and were stable up to 10,000 potential cycles. Similar to the Pt-Ni nanoframes, the improved durability was explained with a reduced Co dissolution, probably achieved because the nanoframes are obtained via corrosion of the dodecahedra using nitric acid, which results in the formation of surfaces with less defects and, consequently, better stability while, in part, compromising activity. ${ }^{182,187}$

Turning to Pt-Fe compounds, very few examples have been reported in the literature compared to Pt-Ni and Pt-Co. Similarly to Pt-Co, structural order was found to be an important parameter for the ORR performance. Kim et al. were able to synthesize chemically disordered face centered cubic $(f c c)$ and chemically ordered face centered tetragonal $(f c t)$ FePt NCs. The latter were obtained by annealing $f c c-\mathrm{FePt} / \mathrm{MgO}$ core/shell $\mathrm{NCs}$ at $750^{\circ} \mathrm{C}$ for $6 \mathrm{~h}$ under forming gas, followed by the shell removal by a dilute acid washing. It is important to note here that the $\mathrm{MgO}$ shell is needed to avoid NC sintering during annealing. The $f c t$-FePt were more active and stable than the $f_{c c}$-FePt when tested for ORR, both with a higher activity than $\mathrm{Pt} / \mathrm{C}$ 
catalysts, thus highlighting the importance of structure in addition to composition. ${ }^{188-191}$ Later, Guo et al. prepared FePt and CoPt NWs with similar dimensions. Both were found to be more active than commercial $\mathrm{Pt} / \mathrm{C}$ and stable over 4,000 potential cycles, however $\mathrm{FePt}$ outperformed CoPt in terms of specific activity. ${ }^{192}$

To summarize this section, Table $\mathbf{S 2}$ showcases the substantial efforts dedicated to this class of NCs as ORR catalysts. Similar to single metal Pt NCs, it is found that the exposure of (111) surfaces combined with a hollow structure are beneficial for ORR, as the Pt-Ni octahedral nanoframes demonstrate the highest specific and mass activities among bimetallic compounds, all superior to the commercial $\mathrm{Pt} / \mathrm{C}$ catalysts tested under the same conditions. Leaching of the non-noble metal remains an issue, however the growth of a Pt skin or doping might be strategies to be pursued in the future.

\subsection{NC alloys of Pt and noble metals as ORR catalysts}

Across the literature, there are also many examples where Pt is combined with other noble metals. While this strategy does not contribute to decreased material costs, higher activity and/or stability is still desirable, especially in alkaline media.

Zhu et al. have shown that a shell of PtIr around facet-controlled Pd cores greatly improves both catalyst activity and durability, thanks to the intrinsically high stability of Ir and activity of Pt in the reaction media. ${ }^{193}$ The same group has then demonstrated that Pt-Ir-Pd nanocages with well-defined (100) facets are very good catalysts towards both ORR and OER in acidic media (Figure 10). ${ }^{194}$ These nanocages demonstrated a strong increase in ORR (4 and 2 times, respectively) and OER (17 and 3 times, respectively) activity compared to both $\mathrm{Pt} / \mathrm{C}$ and $\mathrm{PdPt}$ nanocages, with significantly enhanced durability towards both processes. 

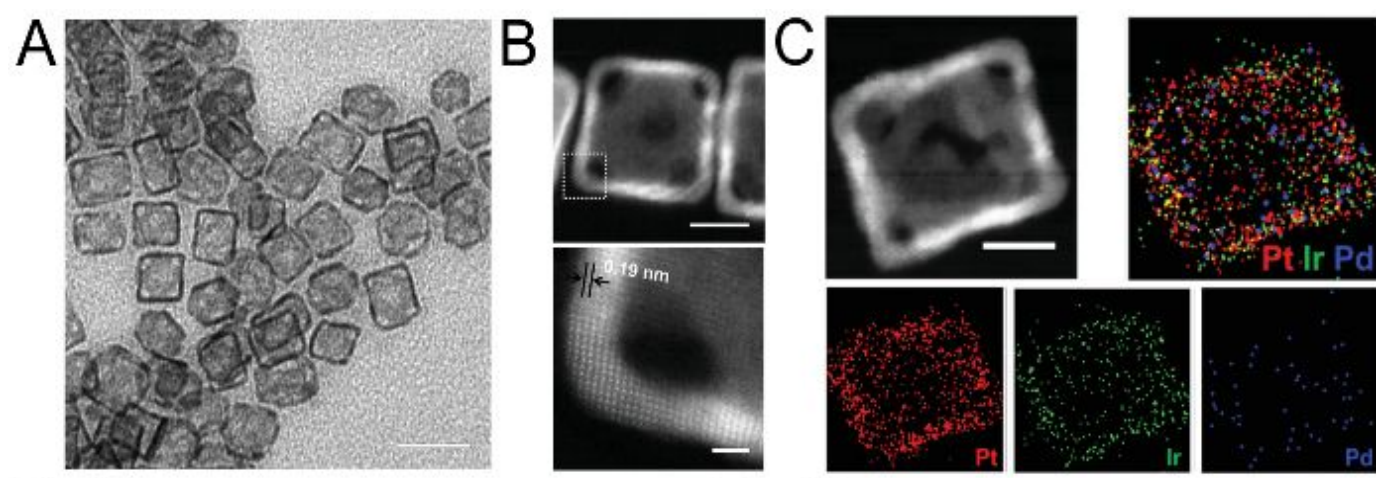

D
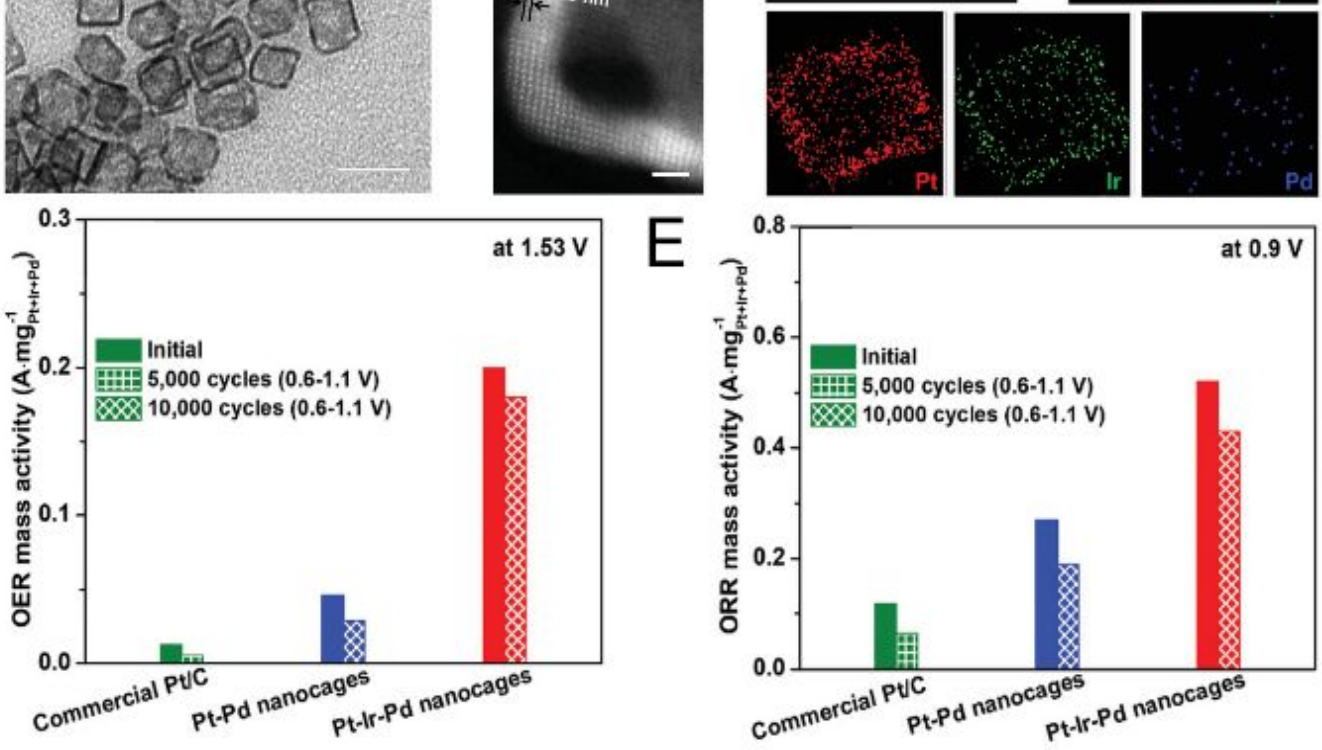

Figure 10. Pt-Ir-Pd as ORR and OER electrocatalysts in acidic conditions. (A) TEM (scale bar $=20 \mathrm{~nm}$ ) and (B) HAADF-STEM images of Pt-Ir-Pd nanocages with single Pt-Ir-Pd particle (top, scale bar $=5 \mathrm{~nm}$ ) and magnified region marked in the top image (bottom, scale bar $=1 \mathrm{~nm}$ ). (C) HAADF-STEM image and EDX mapping of Pt, Ir and Pd for the Pt-Ir-Pd nanocages (scale bar $=5 \mathrm{~nm}$ ). (D) OER and (E) ORR mass activity of Pt-Ir-Pd before and after the accelerated durability test in the ORR potential region $\left(0.6-1.1 \mathrm{~V}_{\mathrm{RHE}}\right)$ in $0.1 \mathrm{M} \mathrm{HClO}_{4}$ in a RDE set-up. Adapted with permission from reference 194. Copyright, 2020 Wiley.

Similar materials design trends can be extended to other noble metals. For example, colloidallysynthesized $f c t$-FePd/Pd, ${ }^{195} \mathrm{PdCu}^{196}$ and five-fold twinned $\mathrm{Pd}_{2} \mathrm{NiAg}^{197}$ catalysts demonstrated higher ORR activity and durability comparable to that of commercial $\mathrm{Pt} / \mathrm{C}$ catalysts.

In case of the $f c t-\mathrm{FePd} / \mathrm{Pd}, \mathrm{Pd}$ lattice compression in the shell induced by the FePd core results in the formation of $\operatorname{Pd}(111)$ surfaces, which are similar to that of $\mathrm{Pt}(111)$. For $\mathrm{PdCu}$, the authors speculate that the activity enhancement is attributed to changes in the electronic structure of the noble metal valence band due to the presence of non-noble metal, which modifies the binding energy of ORR intermediates. Then, in the case of five-fold twinned $\mathrm{Pd}_{2} \mathrm{NiAg}$, the higher activity originates from the increased availability of surface $\mathrm{Ni}$ sites and the features of twinned structural defects. 
Furthermore, Huang et al. have demonstrated that ordered $\mathrm{PdCu}, \mathrm{PdCuNi}$ and PdCuCo NCs synthesized colloidally in a scalable way exhibit bifunctional activity towards ORR in alkaline conditions, which make them particularly interesting. ${ }^{198}$ The PdCuCo mass activity towards ORR is slightly higher than that of $\mathrm{Pt} / \mathrm{C}$ for ORR and the stability is significantly increased. According to DFT calculations, the improved ORR activity originates from the ordered material structure and presence of catalytically active hollow sites of the (111) surface, which arise from the ligand effect and the compressive strain on the Pd surface owing to the smaller atomic size of $\mathrm{Cu}, \mathrm{Co}$, and $\mathrm{Ni}$ atoms.

While promising results have been obtained, the data for this family of NCs as catalysts for ORR are still scarce (Table S2), therefore clear trends and "lessons learned" are not clearly identifiable.

\section{8. $\mathrm{CO}_{2} \mathrm{RR}$}

Electrochemical $\mathrm{CO}_{2} \mathrm{RR}$ into value-added chemicals is an attractive method to mitigate the rising atmospheric $\mathrm{CO}_{2}$ levels while storing renewable energy in a dispatchable manner. ${ }^{199,200}$ Developing catalysts that achieve unit selectivity towards one of the possible reduction products (Table 1) with sustained high rates of formation is among the current challenges in this field that must be addressed at a fundamental level. ${ }^{199,200}$

Table 1. Electrochemical reactions with equilibrium potentials.

$\begin{array}{lll}\text { Reaction } & E^{0} /\left[\mathrm{V}_{\mathrm{RHE}}\right] & \text { (Product) Name, abbreviation } \\ 2 \mathrm{H}_{2} \mathrm{O} \rightarrow \mathrm{O}_{2}+4 \mathrm{H}^{+}+4 \mathrm{e}^{-} & 1.23 & \text { Oxygen evolution reaction, OER } \\ 2 \mathrm{H}^{+}+2 \mathrm{e}^{-} \rightarrow \mathrm{H}_{2} & 0 & \text { Hydrogen evolution reaction, HER } \\ x \mathrm{CO}_{2}+n \mathrm{H}^{+}+n \mathrm{e}^{-} \rightarrow \text { product }+y \mathrm{H}_{2} \mathrm{O} & & \text { CO Reduction Reaction, CO } 2 R \\ \mathrm{CO}_{2}+2 \mathrm{H}^{+}+2 \mathrm{e}^{-} \rightarrow \mathrm{HCOOH}_{(\mathrm{aq})} & -0.12 & \text { Formic acid } \\ \mathrm{CO}_{2}+2 \mathrm{H}^{+}+2 \mathrm{e}^{-} \rightarrow \mathrm{CO}_{(\mathrm{g})}+\mathrm{H}_{2} \mathrm{O} & -0.10 & \text { Carbon monoxide } \\ \mathrm{CO}_{2}+6 \mathrm{H}^{+}+6 \mathrm{e}^{-} \rightarrow \mathrm{CH}_{3} \mathrm{OH}_{(\mathrm{aq})}+6 \mathrm{e}^{-} & 0.03 & \text { Methanol, MeOH } \\ \mathrm{CO}_{2}+4 \mathrm{H}^{+}+4 \mathrm{e}^{-} \rightarrow \mathrm{C}_{(\mathrm{s})}+2 \mathrm{H}_{2} \mathrm{O} & 0.21 & \text { Graphite } \\ \mathrm{CO}_{2}+8 \mathrm{H}^{+}+8 \mathrm{e}^{-} \rightarrow \mathrm{CH}_{4(\mathrm{~g})}+\mathrm{H}_{2} \mathrm{O} & 0.17 & \text { Methane } \\ 2 \mathrm{CO}_{2}+2 \mathrm{H}^{+}+2 \mathrm{e}^{-} \rightarrow\left(\mathrm{COOH}_{2(\mathrm{~s})}\right. & -0.47 & \text { Oxalic Acid } \\ 2 \mathrm{CO}_{2}+8 \mathrm{H}^{+}+8 \mathrm{e}^{-} \rightarrow \mathrm{CH}_{3} \mathrm{COOH}_{(\mathrm{aq})}+2 \mathrm{H}_{2} \mathrm{O} & 0.11 & \text { Acetic Acid } \\ 2 \mathrm{CO}_{2}+10 \mathrm{H}^{+}+10 \mathrm{e}^{-} \rightarrow \mathrm{CH}_{3} \mathrm{CHO}_{(\mathrm{aq})}+3 \mathrm{H}_{2} \mathrm{O} & 0.06 & \text { Acetaldehyde } \\ 2 \mathrm{CO}_{2}+12 \mathrm{H}^{+}+12 \mathrm{e}^{-} \rightarrow \mathrm{C}_{2} \mathrm{H}_{5} \mathrm{COH}_{(\mathrm{aq})}+3 \mathrm{H}_{2} \mathrm{O} & 0.09 & \text { Ethanol, EtOH } \\ 2 \mathrm{CO}_{2}+12 \mathrm{H}^{+}+12 \mathrm{e}^{-} \rightarrow \mathrm{C}_{2} \mathrm{H}_{4(\mathrm{~g})}+4 \mathrm{H}_{2} \mathrm{O} & 0.08 & \text { Ethylene } \\ 2 \mathrm{CO}_{2}+14 \mathrm{H}^{+}+14 \mathrm{e}^{-} \rightarrow \mathrm{C}_{2} \mathrm{H}_{6(\mathrm{~g})}+4 \mathrm{H}_{2} \mathrm{O} & 0.14 & \text { Ethane } \\ 3 \mathrm{CO}_{2}+16 \mathrm{H}^{+}+16 \mathrm{e}^{-} \rightarrow \mathrm{C}_{2} \mathrm{H}_{5} \mathrm{CHO}_{(\mathrm{aq})}+5 \mathrm{H}_{2} \mathrm{O} & 0.09 & \text { Propionaldehyde } \\ 3 \mathrm{CO}_{2}+18 \mathrm{H}^{+}+18 \mathrm{e}^{-} \rightarrow \mathrm{C}_{3} \mathrm{H}_{7} \mathrm{COH}_{(\mathrm{aq})}+5 \mathrm{H}_{2} \mathrm{O} & 0.10 & \text { Propanol, PrOH } \\ x \mathrm{CO}^{+n \mathrm{H}^{+}+n \mathrm{e}^{-} \rightarrow \text { product }}+y \mathrm{H}_{2} \mathrm{O} & & \text { CO Reduction, COR }\end{array}$




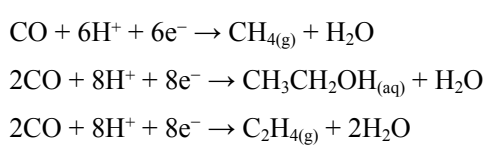

Adapted with permission from reference 199. Copyright 2019, American Chemical Society.

In a seminal contribution uncovering performance trends, Nørskov and co-workers proposed the $2 \mathrm{D}$ volcano plot reported in Figure 11. ${ }^{201}$ Most of the $\mathrm{CO}_{2} \mathrm{RR}$ reaction intermediates bind to metals via carbon atoms. The result is the existence of a linear scaling relation wherein any attempt to increase the reaction rate by stabilizing $\mathrm{H}-\mathrm{CO}^{*}$ stabilizes also $\mathrm{CO}^{*}$ and therefore poisons the catalyst surface. Having the weaker binding energy, Au and Ag are very efficient in producing $\mathrm{CO}$. $\mathrm{Cu}$ is special as it possesses an optimal intermediate binding energy towards the key $\mathrm{CO}_{2} \mathrm{RR}$ intermediates reported in the plot, resulting in "beyond CO" products. 199,200 Nevertheless, polycrystalline $\mathrm{Cu}$ produces over 16 different products, therefore selectivity must be addressed. ${ }^{199,200}$

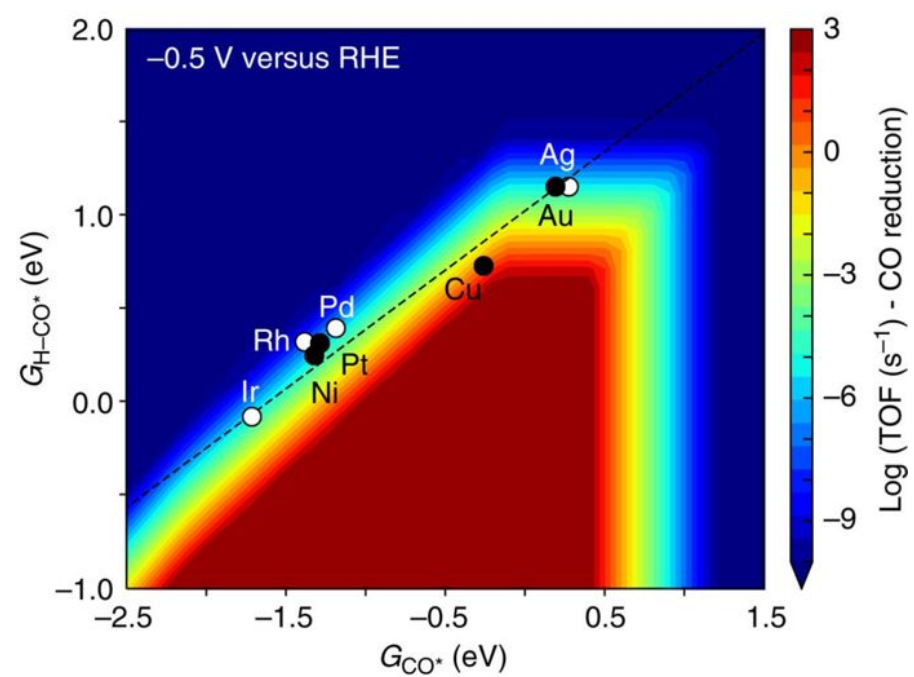

Figure 11. 2D map of the $\mathrm{CO}$ reduction rate as a function of $\mathrm{H}-\mathrm{CO}$ transition state energy and CO binding energy on transition metal surfaces. Adapted with permission from reference 201. Copyright 2017, Nature.

Studies on single crystals have revealed the structural sensitivity of the $\mathrm{CO}_{2} \mathrm{RR}$ with the (100) surface being more selective for ethylene and (111) for methane. ${ }^{199,200,202,203}$ One more recent study has highlighted (751) as a facet promoting $\mathrm{C}-\mathrm{C}$ coupling and oxygenates in particular. ${ }^{204}$ The wide spectrum of $\mathrm{CO}_{2} \mathrm{RR}$ products and the dependence of performance (selectivity, activity and stability) on structure and composition requires careful investigations and tuning of nano-scale surface features. Major facet, facet-ratios, coordination number of active sites, composition, relative distribution of chemically diverse sites and electronic structure influence 
catalytic performance and stability. ${ }^{4}$ Achieving uniformity in these nano-scale features coupled with the possibility of synthesis scale-up to facilitate macro-scale electrode preparation and performance evaluation is exactly the strength of colloidal synthesis.

As discussed for ORR, taking advantage of the tunability offered by colloidal chemistry, monometallic NCs have been exploited to reveal size- and shape-dependent behavior of catalysts in $\mathrm{CO}_{2} \mathrm{RR}$, with most of the studies focused on $\mathrm{Au}$ and $\mathrm{Cu}^{4}$ Building on this knowledge, a few studies on bimetallic NCs have been reported in the literature. Here, the combined effect of multiple binding sites, geometric and electronic effects can result in a deviation from the scaling relations on single metal surfaces. ${ }^{205,206}$ Below, we focus on these two classes of materials and we provide a summary of all the data in Table S3. Compared to HER/HOR and ORR/OER, $\mathrm{CO}_{2} \mathrm{RR}$ has boomed more recently. At the same time, colloidal chemistry has already provided a substantial contribution to the field with facet-controlled $\mathrm{Cu}$ nanocatalysts highly selective towards ethylene and methane. More opportunities open up with the multi-metallic systems, which are still far from being exhaustively explored.

\subsection{Single metal NCs}

While sparse examples have been reported for Pd, Sn and Zn NCs synthesized by wetchemistry routes, ${ }^{207-210}$ most of the studies have focused on size- and shape-controlled Au and $\mathrm{Cu} \mathrm{NCs}$.

In one of the early studies on the size dependence of metal $\mathrm{NCs}$ for $\mathrm{CO}_{2} \mathrm{RR}$, Zhu et al. studied the $\mathrm{CO}_{2} \mathrm{RR}$ performance of colloidally synthesized 4, 6, 8 and $10 \mathrm{~nm} \mathrm{Au} \mathrm{NCs}$ in $\mathrm{CO}_{2}$ saturated $0.5 \mathrm{M} \mathrm{KHCO}_{3}$ (Figure 12). ${ }^{211}$ The authors found a non-monotonic trend with the $8 \mathrm{~nm}$ catalysts reaching a maximum faradaic efficiency (FE) towards $\mathrm{CO}$ up to $90 \%$, which further increased to $97 \%$ by adding an ionic liquid (IL) to the catalyst ink at $-0.67 \mathrm{~V}_{\mathrm{RHE}}$. This behavior was attributed to the stabilization of the ${ }^{*} \mathrm{COOH}$ intermediate due to the optimal ratio between edge (active for $\mathrm{CO}_{2}$ ) and corner (active for $\mathrm{H}_{2}$ ) sites for this size. The same group showed that increasing the number of edge sites by synthesizing ultrathin $\mathrm{Au}$ NWs enhances the performance of $\mathrm{Au}$ nanostructures while also lowering the overpotentials required. ${ }^{212} \mathrm{~A}$ remarkable CO FE of $95 \%$ at only $-0.35 \mathrm{~V}_{\mathrm{RHE}}$ on $2 \mathrm{~nm}$ wide Au NWs was indeed achieved via the stabilized $* \mathrm{COOH}$ and weakened ${ }^{*} \mathrm{CO}$ binding energy. Later on, Mistry et al. exploited the size-dependent behavior of Au NCs in the 1-8 $\mathrm{nm}$ range to tune the composition of the syngas product. $^{213}$ The larger $8 \mathrm{~nm}$ NCs produced a $\mathrm{H}_{2}: \mathrm{CO}$ ratio of $\sim 1: 1$, whereas a $\mathrm{H}_{2}: \mathrm{CO}$ ratio of $\sim 3: 1$ was measured for NCs below $5 \mathrm{~nm}$. DFT calculations showed that the drastic increase in 
HER activity for the smaller NCs is due to possible CO poisoning on the low-coordinated sites that are also active for HER.

A more recent study on $\mathrm{Au}$ single crystals has pointed at a 20 -fold increase in activity for $\mathrm{CO}_{2} \mathrm{RR}$ to $\mathrm{CO}$ on undercoordinated sites, such as those present on $\mathrm{Au}(110)$ and $\mathrm{Au}(211)$, compare to $\mathrm{Au}(100)$ for example. ${ }^{214}$ These findings have inspired researchers to explore $\mathrm{Au}$ NCs with different shapes. In one example, Li et al. have synthesized concave rhombic dodecahedron (RD) Au NCs, which are enclosed by many high-index facets, including (331), (221) and (553). ${ }^{215}$ Their $\mathrm{CO}_{2} \mathrm{RR}$ performance in $0.5 \mathrm{M} \mathrm{KHCO}_{3}$ revealed a striking trend where the concave RDs are superior to regular RDs and cubes in terms of CO FE and current density. The authors hypothesized that the presence of eight different types of high-index facets on the concave RD contributes to this high CO FE of $93 \%$ at $-0.57 \mathrm{~V}_{\mathrm{RHE}}$. In a second example, Liu et al. obtained triangular Ag nanoplatelets exhibiting an ultralow onset potential of $-0.206 \mathrm{~V}_{\mathrm{RHE}}$ in $0.1 \mathrm{M} \mathrm{KHCO}_{3} .{ }^{216}$ DFT calculations showed that the stabilization of the ${ }^{*} \mathrm{COOH}$ on the $\operatorname{Ag}(100)$ facets on the nanoplatelets along with the optimal ratio of edge-to-corners can explain this behavior.
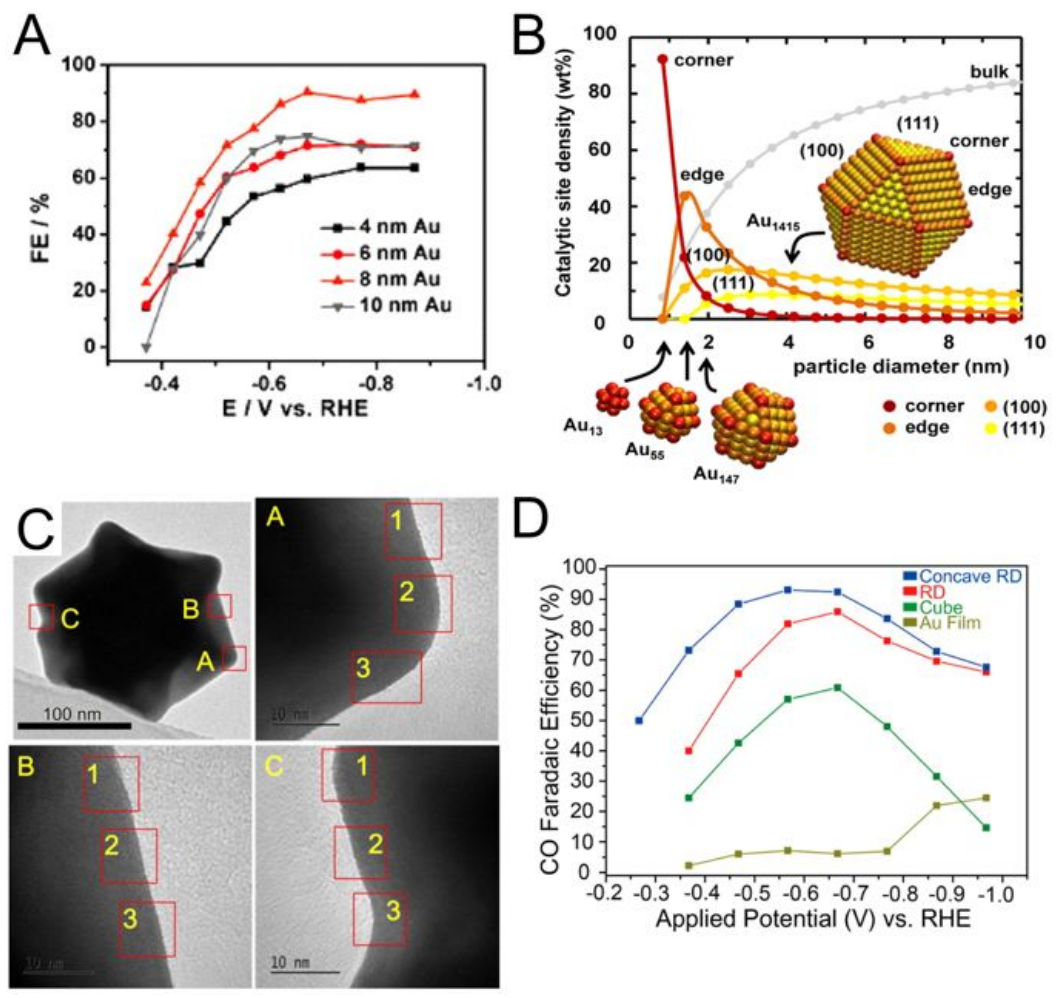

Figure 12. Au NCs as $\mathrm{CO}_{2} \mathrm{RR}$ electrocatalysts. (A) FEs towards $\mathrm{CO}$ for $\mathrm{Au} \mathrm{NCs}$ of different sizes measured in $0.5 \mathrm{M} \mathrm{KHCO}_{3}$. (B) Density of adsorption sites (yellow, light orange, dark orange, or red symbols for (111), (001), edge, or corner on-top sites, respectively) on closedshell cuboctahedral Au clusters vs the cluster diameter. The weight fraction of Au bulk atoms 
is marked with gray dots. Adapted with permission from reference 211. Copyright 2013, American Chemical Society. (C) TEM image of an individual concave RD and magnified images of (A) vertex, (B) edge and (C) bump sections of the same view along the [110] direction. (D) FEs towards $\mathrm{CO}$ for $\mathrm{Au}$ film and $\mathrm{Au} \mathrm{NC}$ electrodes in $0.5 \mathrm{M} \mathrm{KHCO}_{3}$ solution. Adapted with permission from reference 215. Copyright 2015, American Chemical Society.

The number of studies increases substantially when turning to $\mathrm{Cu}$ NCs. As for the sizedependence of the reaction, in one of the early works, $7 \mathrm{~nm} \mathrm{Cu}$ spheres were demonstrated to generate almost $80 \%$ FE towards methane at $-1.3 \mathrm{~V}_{\text {RHE }}$ while evolving into $25 \mathrm{~nm}$ particles in $0.1 \mathrm{M} \mathrm{NaHCO}_{3}$ (Figure 13A,B). ${ }^{217}$ Reske et al. investigated $\mathrm{Cu}$ spheres in the size range of $1.9 \mathrm{~nm}$ to $15.1 \mathrm{~nm}$ and observed that these catalysts produce mostly hydrogen (Figure 13C). ${ }^{218}$ Such behavior was explained by attributing HER to the low-coordinate sites which are abundant on the surface of small particles (Figure 13D). This study clearly shows the importance of avoiding very small $\mathrm{Cu} \mathrm{NCs}$ as $\mathrm{CO}_{2} \mathrm{RR}$ catalysts, something which was confirmed also by later investigations. ${ }^{219}$ Possible explanation of the disagreement between the results of these two works might reside in the particle coverage on the electrodes. Indeed, interparticle distance was later on demonstrated to be an important parameter due to the desorption and readsorption of intermediates on neighboring NCs. ${ }^{39}$ The fact that the two catalysts were synthesized by different chemical approaches might also play a role.
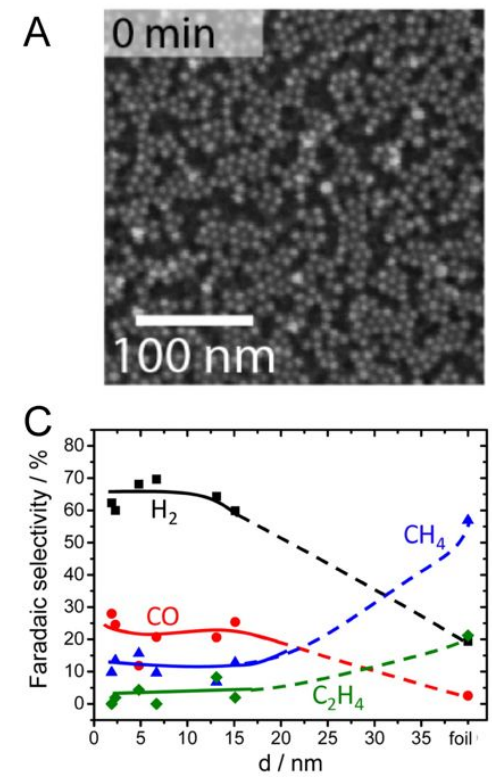

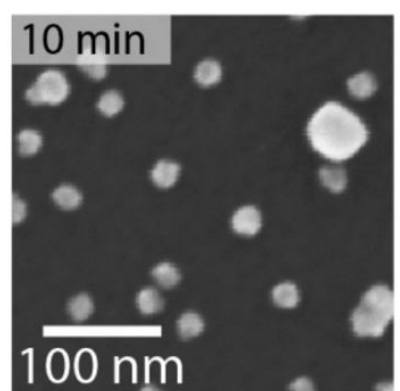

$\mathrm{D}$

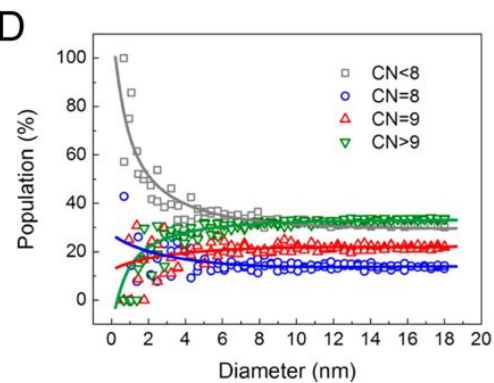

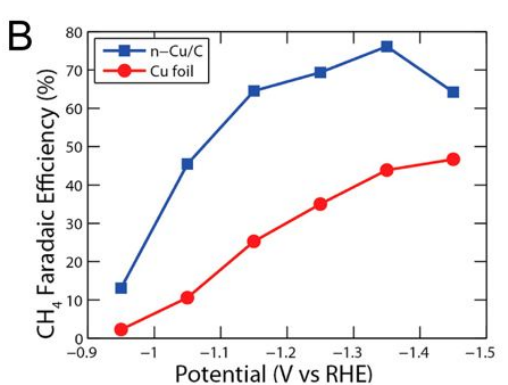

$E$

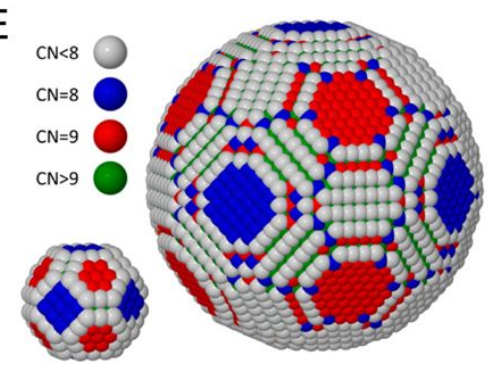

Figure 13. Size-controlled spherical $\mathrm{Cu}$ NCs for $\mathrm{CO}_{2}$ RR. (A) Scanning electron microscopy (SEM) images of the spherical $\mathrm{Cu}$ NCs supported on glassy carbon and the same after $10 \mathrm{~min}$ 
of electroreduction at $-1.25 \mathrm{~V}_{\mathrm{RHE}}$ in $0.1 \mathrm{M} \mathrm{NaHCO}_{3}$. (B) FEs towards $\mathrm{CH}_{4}$ on glassy carbon supported $\mathrm{Cu} \mathrm{NCs}$ and $\mathrm{Cu}$ foil electrodes in $0.1 \mathrm{M} \mathrm{NaHCO}_{3}$. Adapted with permission from reference 217. Copyright 2014, American Chemical Society. (C) FEs of Cu NCs of different sizes and $\mathrm{Cu}$ foil electrodes measured at $-1.1 \mathrm{~V}_{\mathrm{RHE}}$ in $0.1 \mathrm{M} \mathrm{KHCO}_{3}$. (D) Relative ratio of surface atoms having a specific $\mathrm{CN}$ as a function of particle diameter. (E) Ball models of spherical $\mathrm{Cu}$ NCs with 2.2 and $6.9 \mathrm{~nm}$ diameters. Surface atoms are color-coded according to their first neighbor coordination number $(\mathrm{CN}), \mathrm{CN}<8$ (gray), $\mathrm{CN}=8$ (blue), $\mathrm{CN}=9$ (red), CN $>9$ (green). Adapted with permission from reference 218. Copyright 2014, American Chemical Society.

As mentioned above, studies on $\mathrm{Cu}$ single crystals have evidenced the structure-dependence of the $\mathrm{CO}_{2} \mathrm{RR}$ selectivity. ${ }^{202,203}$ Consequently, tuning the $\mathrm{Cu} \mathrm{NC}$ shapes is an appealing strategy to reduce the number of products obtained on $\mathrm{Cu}$ catalysts. Loiudice et al. have synthesized $\mathrm{Cu}$ nanocubes with edge lengths of $24 \mathrm{~nm}, 44 \mathrm{~nm}$ and $63 \mathrm{~nm}$ (Figure 14A) ${ }^{219}$ In addition to the promotion of ethylene, which was expected from single crystal studies, an interesting sizedependent behavior was observed. Indeed, the $44 \mathrm{~nm} \mathrm{Cu}$ cubes exhibited the best performance by achieving a maximum FE towards ethylene of $41 \%$ at $-1.1 \mathrm{~V}_{\mathrm{RHE}}$ (Figure 14B). This behavior was explained with the optimal ratio between (100) facets and (110) edges. Indeed, DFT calculations confirmed a dual facet mechanism wherein the $\mathrm{C}-\mathrm{C}$ coupling reaction leading to ethylene formation occurs at the (100)/(110) interface (Figure 14C). ${ }^{220}$ Interestingly, cube-like $\mathrm{Cu}$ particles in a similar size range $(10-40 \mathrm{~nm})$ but forming during the first stages of $\mathrm{CO}_{2} \mathrm{RR}$ from $8 \mathrm{~nm} \mathrm{Cu}$ spheres when loaded at high concentration on the electrodes, exhibited a higher selectivity for $\mathrm{C}_{2}+\mathrm{C}_{3}$ products compared to the as-synthesized $\mathrm{Cu}$ nanocubes. ${ }^{221} \mathrm{Such}$ result indicates that unique catalytic sites might be forming during in situ transformations.

In another study, Iyengar et al. studied the size-dependent behavior of $\mathrm{Cu}$ octahedra as $\mathrm{CO}_{2} \mathrm{RR}$ catalysts (Figure 14D). ${ }^{64} \mathrm{~A}$ striking HER suppression and $\mathrm{CO}_{2} \mathrm{RR}$ promotion was observed as the size of the octahedra decreased from $310 \mathrm{~nm}$ to $75 \mathrm{~nm}$ (Figure 14E). Indeed, the latter were the most selective for $\mathrm{CO}_{2} \mathrm{RR}$ and achieved a maximum $\mathrm{CH}_{4} \mathrm{FE}$ of $55 \%$ at $-1.25 \mathrm{~V}_{\mathrm{RHE}}$. The higher contribution of corners and edges to the overall surface area in the smaller $\mathrm{Cu}$ octahedra, as quantified by the $(100) /(111)$ and $(110) /(111)$ ratios, can be identified as the reason for this dependence. 

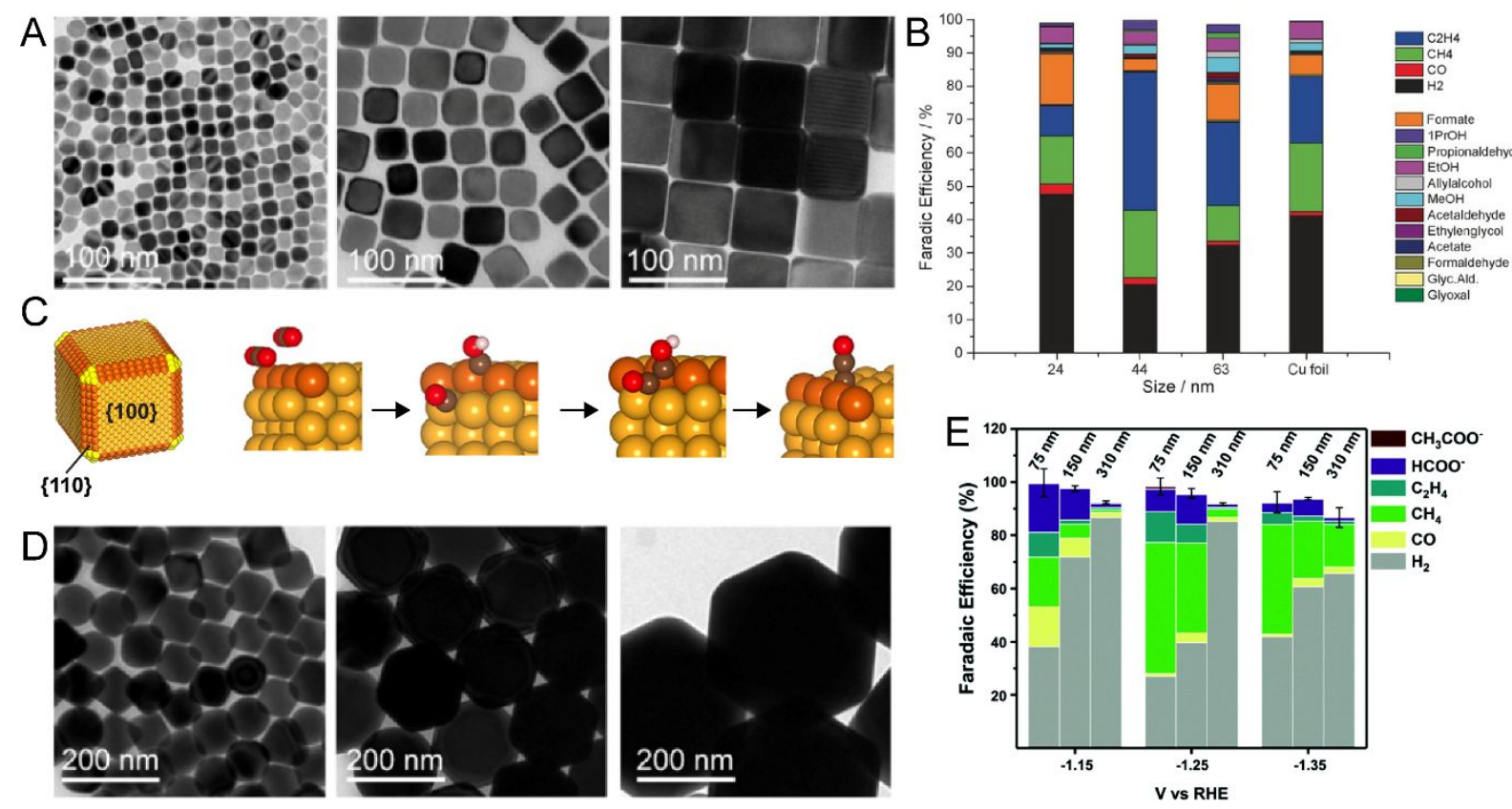

Figure 14. Shape-controlled $\mathbf{C u}$ NCs for $\mathbf{C O}_{2}$ RR. (A) Representative TEM images of cubic $\mathrm{Cu}$ NCs with three sizes, namely $24 \mathrm{~nm}, 44 \mathrm{~nm}$ and $60 \mathrm{~nm}$. (B) FEs for reaction products at $-1.1 \mathrm{~V}_{\mathrm{RHE}}$ in $0.1 \mathrm{M} \mathrm{KHCO}_{3}$ from the same $\mathrm{NCs}$ and $\mathrm{Cu}$ foil for comparison. Adapted with permission from ref 219. Copyright 2016, Wiley. (C) Schematic illustrating the dual facet mechanism for $\mathrm{CO}_{2} \mathrm{RR}$ on cubic $\mathrm{Cu}$ NCs. Adapted with permission from reference 220 . Copyright 2019, American Chemical Society. (D) TEM images of $75 \mathrm{~nm}, 150 \mathrm{~nm}$ and $310 \mathrm{~nm}$ Octahedral $\mathrm{Cu}$ NCs. (E) Potential dependent FEs for reaction products from the same in $0.1 \mathrm{M}$ $\mathrm{KHCO}_{3}$. All samples were deposited on glassy carbon substrates. Adapted with permission from reference 64. Copyright 2019, Royal Society of Chemistry.

A similar FE for methane $\left(55 \%\right.$ at $-1.25 \mathrm{~V}_{\mathrm{RHE}}$ in $\left.0.1 \mathrm{M} \mathrm{KHCO}_{3}\right)$ was obtained by Lin et al. using thin pentatwinned $\mathrm{Cu}$ NWs with a diameter of $20 \mathrm{~nm} .{ }^{222}$ The highly strained edges at the twinning boundaries of the pentatwinned NWs were proposed to be the catalytically active sites. Finally, Chen et al. observed a remarkably high selectivity for ethanol (FE of $25 \%$ at -1.2 $\mathrm{V}_{\mathrm{RHE}}$ ) on the hitherto unexplored morphology of hexarhombic dodecahedral $\mathrm{Cu} \mathrm{NCs}^{223}$ The unexpected $\mathrm{C}_{2} \mathrm{H}_{5} \mathrm{OH}$ selectivity was attributed to the larger proportion of $\mathrm{Cu}(110)$ edge sites and DFT calculations revealed that the mechanistic bifurcation between the $\mathrm{C}_{2} \mathrm{H}_{4}$ and $\mathrm{C}_{2} \mathrm{H}_{5} \mathrm{OH}$ pathways may be caused by the lower binding energy of adsorbed $* \mathrm{O}$ on these sites.

To summarize, mono-metallic colloidal $\mathrm{NC}$ catalysts studied so far for $\mathrm{CO}_{2} \mathrm{RR}$ uncovered important general trends and we refer to Table S3 for details. Firstly, metal catalysts smaller than $15 \mathrm{~nm}$ favor the HER over $\mathrm{CO}_{2} \mathrm{RR}$, which is explained as they have a large number of 
low-coordinated sites, as seen by studies on Au and Cu NCs. An optimal size appears to be around in the range 40-80 nm. Secondly, in agreement with single crystal studies, the shape, which controls the dominant surfaces exposed, governs selectivity (i.e. cubes are highly selective for ethylene, octahedra for methane). For NCs with well-defined shape, the size is also important as it controls the facet ratio, a property shown to have a profound impact on the performance and stability of NCs. An optimum in the terrace vs step-edge sites on NCs is an unanimously agreed upon strategy to suppress the competing HER (i.e. around $40 \mathrm{~nm}$ for cubes). It is also interesting to note that generally no ligand removal treatment is applied for these catalysts, which is related to the observation of electro-stripping occurring in many cases. ${ }^{88}$ Nevertheless, more attention to this ligand effects should be given in the future. Commenting on intrinsic activities is not trivial as a common method to determine the active surface area is still lacking at this point.

\subsection{Cu-based bimetallic NCs}

To date, most of the studies employing colloidal chemistry as tool for catalyst design have focused on the coupling of two metals on top of the volcano plot in Figure 11, that is Cu with the CO-evolving Au and Ag. ${ }^{224}$

By using a co-reduction method, Kim et al. have synthesized spherical $11 \mathrm{~nm} \mathrm{AuCu}$ alloys of varying composition $\left(\mathrm{Au}_{3} \mathrm{Cu}, \mathrm{AuCu}, \mathrm{AuCu}_{3}\right)$ and tested them as monolayers on glassy carbon electrodes (Figure 15A). ${ }^{40} \mathrm{Here}, \mathrm{Au}_{3} \mathrm{Cu}$ was found as the most active toward $\mathrm{CO}$ production (Figure 15B). The obtained activity trend was explained as a result from the combination of structural and electronic effects enabling an optimized $\mathrm{COOH}^{*}$ intermediate binding energy. In a follow-up study, the same group investigated the impact of the atomic ordering on the $\mathrm{CO}_{2} \mathrm{RR}$ performance by synthesizing $\mathrm{AuCu}$ disordered alloys, progressively ordered alloys and an intermetallic species (Figure 15C). ${ }^{225}$ The catalysts were progressively more CO selective with increasing degree of ordering reaching the highest intrinsic activity for the intermetallic o_AuCu (Figure 15D). Eventually, HRTEM investigations showed that a three-atom thick overlayer of Au was present on the surface of these particles. DFT calculations confirmed that the compressive strain induced in the Au overlayer by the core boosted the intrinsic activity of $\mathrm{Au}$ and was therefore the origin of the enhanced intrinsic activity. A different approach to varying the composition of $\mathrm{Au}-\mathrm{Cu} \mathrm{NCs}$ was implemented by Zhu et al. ${ }^{226}$ The authors investigated the dealloying effect in $\mathrm{Au}_{3} \mathrm{Cu}$ NCs. The selective etching of surface $\mathrm{Cu}$ atoms by nitric acid treatment was found to increase the FE and intrinsic activity towards CO. DFT 
calculations pointed towards a more facile $\mathrm{COOH}^{*}$ formation on vacancy adjacent $\mathrm{Au}$ atoms explaining the enhanced performance.

A very interesting study was reported by Tao et al. 227 Here, nanocomposite electrodes consisting of $\mathrm{Au} \mathrm{NCs}$ embedded in a $\mathrm{Cu}$ matrix were prepared by electrochemically reducing a physical mixture of $\mathrm{Au}$ and $\mathrm{CuO}$ NCs at different ratios. While most studies report $\mathrm{Au}-\mathrm{Cu}$ catalysts to be selective towards $\mathrm{CO}$, these catalysts were capable to produce formate with a FE as high as $79 \%$ at a total current density of $\sim 11 \mathrm{~mA} / \mathrm{cm}^{2}$ for $4 \mathrm{~h}$. Metal-metal interactions were proposed to induce the disappearance of Au's characteristic electrocatalytic activity for reducing $\mathrm{CO}_{2}$ to $\mathrm{CO}$ and to stabilize $\mathrm{Cu}^{+}$species on the $\mathrm{Cu}$ surface at $\mathrm{CO}_{2}$ reduction potentials, associated with formate production. While the selectivity towards formate remained unexplained, surely this study highlights the importance of the metal distribution within the $\mathrm{Au}-\mathrm{Cu}$ bimetallic catalysts to direct selectivity.
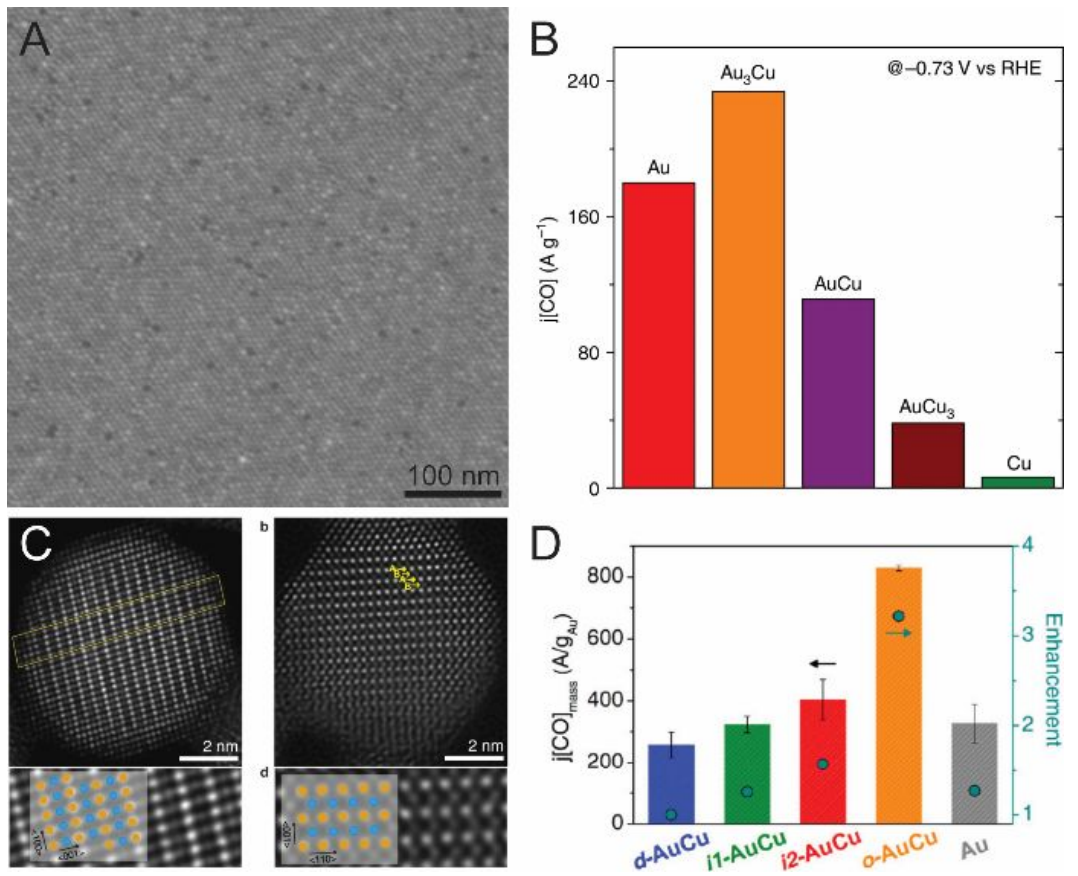

Figure 15. Au-Cu NCs for $\mathbf{C O}_{2}$ RR. (A) SEM image of $\mathrm{AuCu}_{3} \mathrm{NCs}$ monolayer and (B) specific mass activities at $-0.73 \mathrm{~V}_{\mathrm{RHE}}$ in $\mathrm{CO}_{2}$ saturated $0.1 \mathrm{M} \mathrm{KHCO}_{3}$ electrolyte of $\mathrm{Au}-\mathrm{Cu}$ NCs with various compositions. Adapted with permission from reference 40. Copyright 2014, Nature. (C) HRTEM analysis of intermetallic AuCu NCs. (D) CO mass activities at $-0.77 \mathrm{~V}_{\mathrm{RHE}}$ in $\mathrm{CO}_{2}$ saturated $0.1 \mathrm{M} \mathrm{KHCO}_{3}$ electrolyte of increasingly ordered $\mathrm{AuCu}$ alloys: d-AuCu, i1$\mathrm{AuCu}, \mathrm{i} 2-\mathrm{AuCu}$ and o-AuCu. Adapted with permission from reference 225. Copyright 2017, American Chemical Society. 
Differently than $\mathrm{Au}-\mathrm{Cu}, \mathrm{Ag}-\mathrm{Cu}$ are immiscible over the entire range of their binary phase diagram. For this reason, Huang et al. could synthesize $\mathrm{Ag}-\mathrm{Cu}$ nanodimers with different $\mathrm{Cu}$ domain sizes, without alloying occurring (Figure 16A). ${ }^{27}$ The $\mathrm{Ag}_{1} \mathrm{Cu}_{1.1}$ dimers, possessing the largest interface between $\mathrm{Ag}$ and $\mathrm{Cu}$, were found to be the best in promoting $\mathrm{C}-\mathrm{C}$ coupling when compared to the dimers with different compositions, to the $\mathrm{Cu}$ and Ag NCs as standalone catalysts and also to the physical mixture of $\mathrm{Ag}$ and $\mathrm{Cu} \mathrm{NCs}$, both in terms of FE and mass activity (Figure 16B). This behavior was attributed to the combination of tandem catalysis (Ag producing $\mathrm{CO}$ and $\mathrm{Cu}$ converting it further) and modified electronic structure due to the observed charge-transfer from the $\mathrm{Cu}$ to the $\mathrm{Ag}$ domain (Figure 16C). Interestingly, the comparison of these results with those obtained from surface alloyed $\mathrm{Cu}-\mathrm{Ag}$ thin films, that promote multi-carbon oxygenated products, or $\mathrm{Cu}-\mathrm{Ag}$ microelectrodes, which promote $\mathrm{C}_{2} / \mathrm{C}_{3}$ products but not one in particular, suggest that this system also is sensitive to the metal distribution within the catalysts and perhaps to the length scale at which the metals mix. ${ }^{228,229}$ 


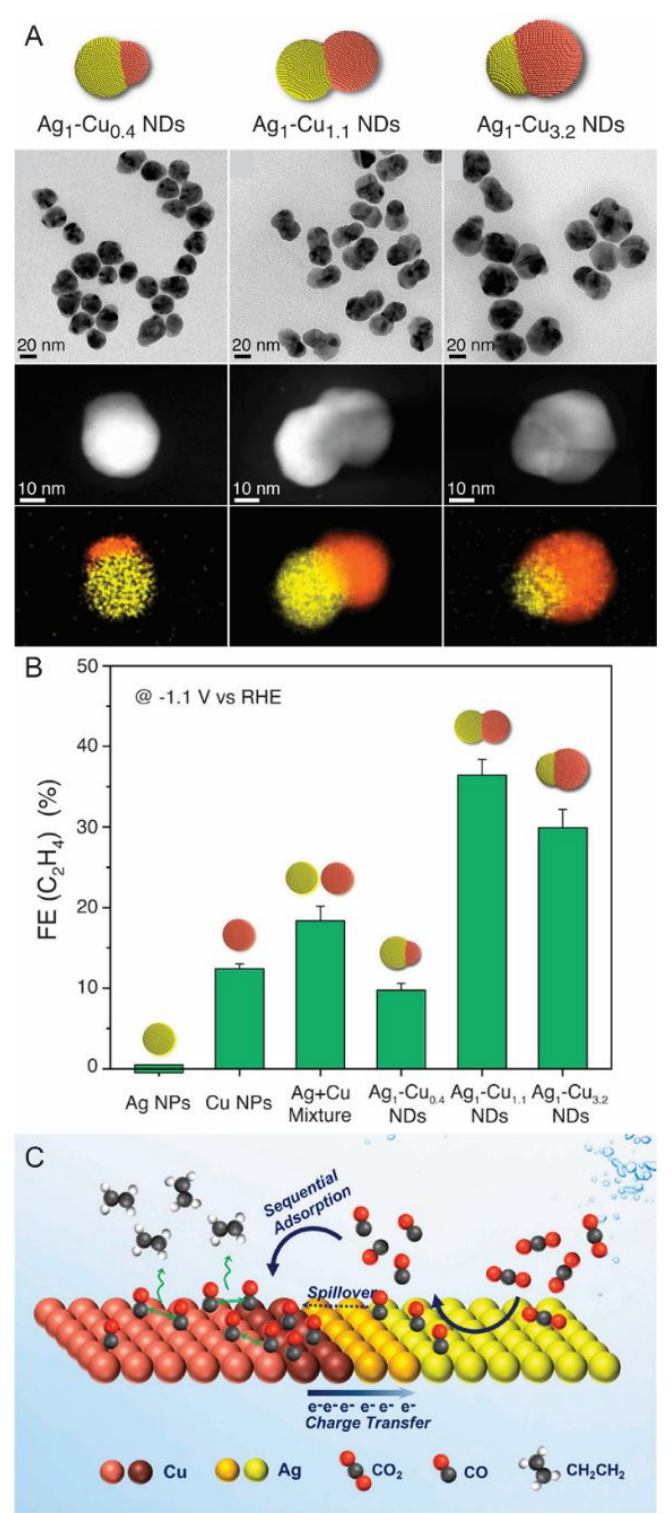

Figure 16. Ag-Cu NCs for $\mathbf{C O}_{2}$ RR. (A) Schemes, TEM and HAADF-STEM images along with EDX elemental maps of $\mathrm{Ag}_{1} \mathrm{Cu}_{0.4}, \mathrm{Ag}_{1} \mathrm{Cu}_{1.1}$ and $\mathrm{Ag}_{1} \mathrm{Cu}_{3.2}$ nanodimers. (B) FE towards ethylene at $-1.1 \mathrm{~V}_{\mathrm{RHE}}$ in $0.1 \mathrm{M} \mathrm{KHCO}_{3}$. (C) Sketch of the proposed mechanism for the enhanced $\mathrm{C}-\mathrm{C}$ coupling. Adapted with permission from reference 27. Copyright 2019, American Chemical Society.

Finally, one bimetallic system colloidally prepared for which more than one example have been reported in the literature is Cu-Pd. Wang et al. synthesized Pd@Cu core@shell NCs with different shapes exposing high energy facets, i.e. regular cubes, truncated cubes and rhombic dodecahedrons (Figure 17A). ${ }^{230}$ They found that the increased density of highly-stepped (110) facets on the rhombic dodecahedrons led to a significantly increased $\mathrm{CO}_{2} \mathrm{RR}$ activity (33.6 $\mathrm{mA} / \mathrm{cm}^{2}$ compared to $14.2 \mathrm{~mA} / \mathrm{cm}^{2}$ for the as-synthesized $\mathrm{Pd} @ \mathrm{Cu}$ cubes, at 
$-1.4 \mathrm{~V}_{\mathrm{Ag} / \mathrm{AgCl}}$ ) with a high FE towards methane up to nearly $60 \%$ (Figure 17B). In another example on $\mathrm{Cu}-\mathrm{Pd} \mathrm{NCs}$, Ma et al. prepared phase separated, disordered and ordered catalysts (Figure 17C) and studied the effect of phase and order on the electrocatalytic properties. ${ }^{231} \mathrm{~A}$ substantial promotion of $\mathrm{CO}_{2} \mathrm{RR}$ and particularly of ethylene production was found in the phase segregated catalysts (Figure 17D). The authors proposed that the increased number of $\mathrm{Cu}-\mathrm{Pd}$ bonds in the intermetallic and disordered species reduces the $\mathrm{C}-\mathrm{C}$ coupling rate and thereby enhances the selectivity for $\mathrm{CO}$. Instead, preserving the $\mathrm{Cu}$ identity in the phase segregated catalysts helps to promote the $\mathrm{C}-\mathrm{C}$ coupling, though probably through different reaction pathways due to the presence of an interface with an element with a higher oxygen affinity. Although colloidal methods were not specifically used by Ma et al. for the synthesis, the comparison of the two studies on $\mathrm{Cu}-\mathrm{Pd} \mathrm{NCs}$ as catalysts for $\mathrm{CO}_{2} \mathrm{RR}$ is interesting as it reveals once again that the synthetic approach and distribution of metals within the catalysts are both extremely important. 

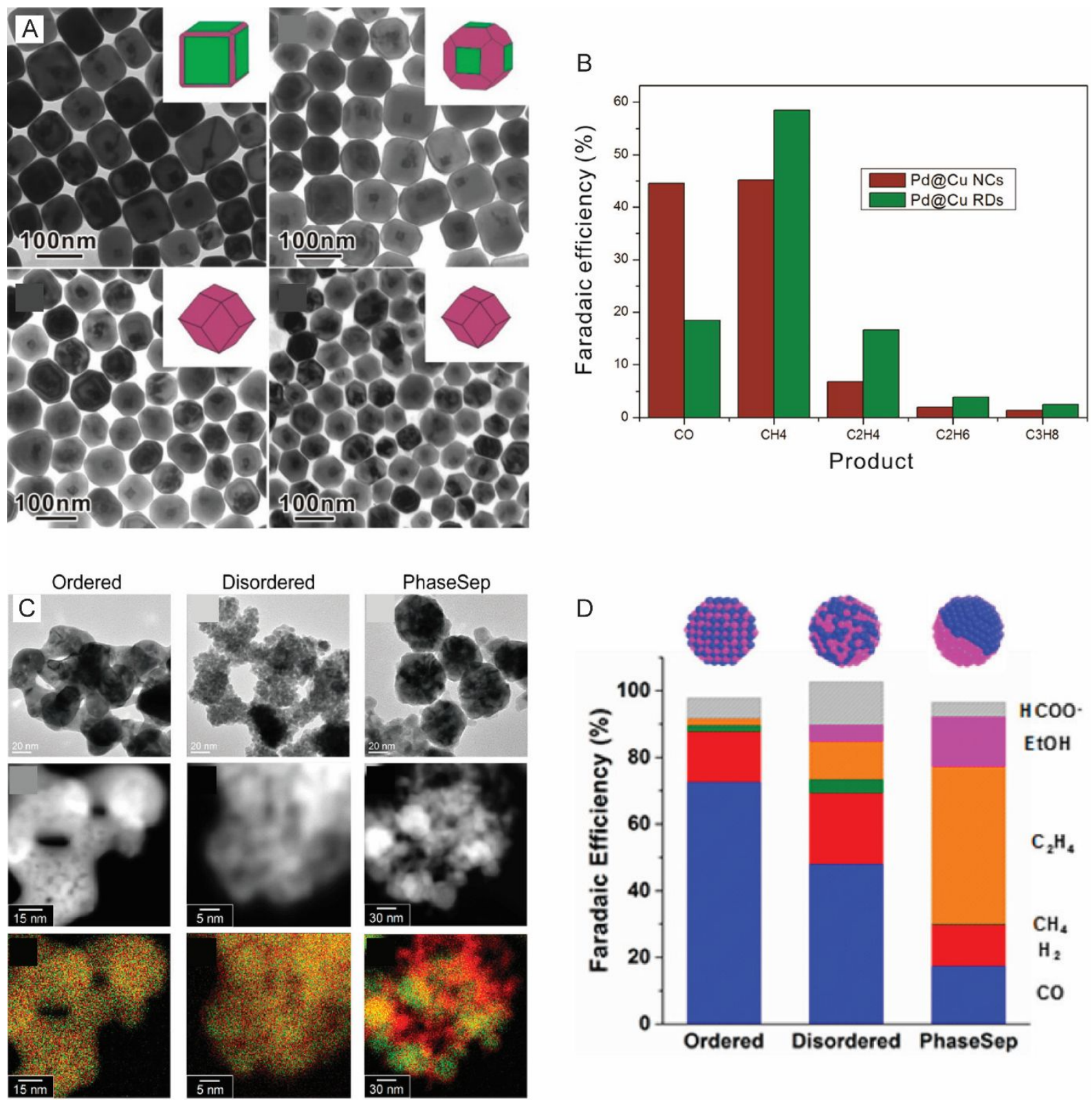

Figure 17. Pd-Cu NCs for $\mathbf{C O}_{2}$ RR. (A) TEM images of the as-synthesized core@shell $\mathrm{Pd} @ \mathrm{Cu}$ nanocubes, truncated cubes, rhombic dodecahedrons and size reduced rhombic dodecahedrons. (B) FEs of the same measured at $-1.4 \mathrm{~V}_{\mathrm{Ag} / \mathrm{AgCl}}$. Adapted with permission from reference 230. Copyright 2016, American Chemical Society. (C) TEM images, HAADFSTEM and EDX elemental maps of ordered-Cu-Pd, disordered-Cu-Pd and phase-segregated $\mathrm{Cu}-\mathrm{Pd}$ NCs. The solid-solution type and phase-segregated catalysts were synthesized using a co-reduction method. The ordered intermetallic species was obtained through the annealing of the disordered system at $300{ }^{\circ} \mathrm{C}$ for $3 \mathrm{~h}$ in $0.1 \mathrm{MPa} \mathrm{H}_{2}$. (D) FEs at $-0.76 \mathrm{~V}_{\mathrm{RHE}}$ in $1 \mathrm{M} \mathrm{KOH}$ electrolyte. Adapted with permission from reference 231. Copyright 2017, American Chemical Society. 
In summary, while the overall number of studies on colloidal bimetallic $\mathrm{NCs}$ for $\mathrm{CO}_{2} \mathrm{RR}$ is still limited (Table S3), it is evident that in addition to composition, the catalytic outcome is dictated also by the relative size and spatial arrangement of the constituent metals. This effect is evident in $\mathrm{CO}_{2} \mathrm{RR}$ more than in other reactions because, in addition to electronic effects discussed in the case of Ni-Pt for ORR, bimetallic systems display the capability of hosting tandem schemes, where intermediates from one surface (i.e. $\mathrm{CO}$ on $\mathrm{Au}, \mathrm{Ag}, \mathrm{Pd}$ ) moves on the neighboring chemically distinct domain $(\mathrm{Cu})$ to react further. Therefore, having an alloyed, intermetallic or phase segregated bimetallic NCs can play a big role in favoring one mechanism over the other, thus impacting the product distribution. Future studies to systematically investigate these relations would be helpful. Additionally, all these phenomena could possibly be leveraged vis-à-vis the structure-property relations shown by mono-metallic NCs (i.e. shape dependence) to uncover useful information in future studies.

\section{Anodic fuel-cell reactions}

Whilst $\mathrm{H}_{2}$ can be used in the anodic reaction process of fuel cells, liquid fuels hold many benefits as they are more energy dense and easier to transport. Developing the oxidation reactions of formic acid (FAOR), methanol (MOR), ethylene glycol (EGOR), ethanol (EOR) and glycerol (GOR) is therefore an important challenge. ${ }^{232}$ The anodic half-reactions for all of these processes are given below. Ethanol and glycerol are particularly appealing fuels, as they can be obtained from biomass and are less toxic, volatile, flammable and corrosive than methanol or formic acid. For energy-based applications, complete oxidation to $\mathrm{CO}_{2}$ is desirable.

$$
\begin{aligned}
& \mathrm{HCO}_{2} \mathrm{H} \rightarrow 2 \mathrm{H}^{+}+2 e^{-}+\mathrm{CO}_{2}(\mathrm{FAOR}) \\
& \mathrm{CH}_{3} \mathrm{OH}+\mathrm{H}_{2} \mathrm{O} \rightarrow 6 \mathrm{H}^{+}+6 e^{-}+\mathrm{CO}_{2}(\mathrm{MOR}) \\
& \left(\mathrm{CH}_{2} \mathrm{OH}\right)_{2}+2 \mathrm{H}_{2} \mathrm{O} \rightarrow 10 \mathrm{H}^{+}+10 e^{-}+2 \mathrm{CO}_{2}(\mathrm{EGOR}) \\
& \mathrm{CH}_{3} \mathrm{CH}_{2} \mathrm{OH}+3 \mathrm{H}_{2} \mathrm{O} \rightarrow 12 \mathrm{H}^{+}+12 e^{-}+2 \mathrm{CO}_{2}(\mathrm{EOR}) \\
& \mathrm{HOCH}_{2} \mathrm{CH}(\mathrm{OH}) \mathrm{CH}_{2} \mathrm{OH}+3 \mathrm{H}_{2} \mathrm{O} \rightarrow 14 \mathrm{H}^{+}+14 e^{-}+3 \mathrm{CO}_{2}(\mathrm{GOR})
\end{aligned}
$$

Noble metals such as $\mathrm{Pt}$ and Pd show the highest activities in these reactions and have been studied extensively. ${ }^{8,233}$ However, ${ }^{*} \mathrm{CO}$ is a common intermediate during the oxidation pathway, and its strong binding to PGMs (Figure 11) is a frequent cause of poisoning and 
deactivation. Manipulating the reaction mechanism to avoid this intermediate is therefore a major challenge for catalyst design, together with substituting Pt and Pd with more Earthabundant elements. In reactions involving $\mathrm{C}_{2+}$ alcohols, the complete oxidation involves $\mathrm{C}-\mathrm{C}$ bond cleavage, representing an additional challenge. ${ }^{8,234}$ A structure sensitivity has been shown for many of these anodic reactions with the ad-hoc combination of dominant surface facet and high-index planes and defect sites leading to high activities. ${ }^{8}$ It is also well documented that $\mathrm{CO}$ intermediates can be avoided by using alloys. ${ }^{8}$ Below, we discuss the different studies across the literature organized by reaction as the compositional diversity of the investigated NCs did not allow to follow the same organization of the previous sections. All the relative data are summarized in Tables S4-S7. The results discussed in this part especially highlight that the morphological complexity achievable by colloidal chemistry has a huge potential in the discovery of new electrocatalysts in addition to the more trivial translation of knowledge from single crystal studies to more realistic conditions.

\subsection{Formic acid oxidation.}

While the reaction mechanisms are less clear, one of the advantages of Pd over Pt in the FAOR is that it seems more tolerant to CO poisoning. ${ }^{235}$ As such, well-defined colloidal Pd NCs have been employed extensively in the FAOR. ${ }^{236-245}$ A wide variety of shapes have been studied, including cubes, ${ }^{241-243}$ octahedra $^{241,243}$, wires, ${ }^{238,239}$ decahedra, ${ }^{243}$ right bipyramids, ${ }^{237,243}$ tetrahedra ${ }^{243}$ and icosahedra, ${ }^{240,243}$ which has consequently permitted important insight into the dependence of the FAOR performance on particular surface sites. Comparing shapes dominated by low-index facets, Choi et al. showed that FAOR activity is generally higher on (100) facets than on (111) facets (e.g. Pd cubes are more active than Pd octahedra). ${ }^{243}$ In a different study from the same group, the FAOR activity was shown to be proportional to the amount of exposed (100) facets. A series of Pd NC catalysts were prepared, ranging from cubes, cuboctahedra and octahedra, representing decreasing amounts of exposed (100) facets (Figure 18A). Linear correlations were identified, where (100) facets promoted the highest FAOR activities, but (111) facets exhibited the lowest peak potentials (Figure 18B). ${ }^{241}$ Zheng et al. identified that $\operatorname{Pd}(100)$ facets can accommodate two bridge-formate intermediates, explaining the higher activity on these facets. ${ }^{246}$

The presence of higher-index (211) twin-defect sites on low-index Pd NCs further improves their activity by altering the energetics of reaction intermediates such that the poisoning $\mathrm{CO}$ intermediate is avoided. DFT calculations revealed $\mathrm{COOH}$ and $\mathrm{HCOO}$ are isoenergetic on the (211) sites, meaning that these sites remain free of $\mathrm{CO}$ during the reaction, leading to higher 
activities. ${ }^{243}$ Therefore, right-bipyramids, containing (100) facets and (211) defect sites, are much more active than cubes, which contain only (100) facets. Similarly, icosahedra, containing many (111) and (211) facets, are much more active than (111)-containing tetrahedra, and are also more active than the (100)-containing cubes. Such twin-defect sites and other high-index facets are highly active in the FAOR, which has been exploited through the rational synthesis of concave NCs, including tetrahedra, ${ }^{247}$ trigonal bipyramids, ${ }^{247}$ decahedra, ${ }^{248}$ rods, ${ }^{249}$ right-bipyramids ${ }^{249}$ and cubes. ${ }^{242,249}$ In the case of high-index facets on concave faces, improved catalytic performance is often rationalized by the presence of atomic kink and step sites. With a high degree of control, Wang et al. showed that both FAOR activity and stability increased in the series: $\mathrm{Pd} / \mathrm{C}<\mathrm{Pd}$ nanocubes $<\mathrm{Pd}$ twinned $\mathrm{NCs}<$ concave $\mathrm{Pd}$ nanocubes $<$ concave Pd twinned NCs, illustrating the strength of combining twin-defects and high-index facets. ${ }^{249}$

Multimetallic nanocatalysts have proven to be highly active in the FAOR. Bearing in mind the importance of high-index facets emerged from studies on Pd NCs, Lee et al. intricately prepared a series of polyhedral Au@Pd NCs possessing high-index facets, including trisoctahedra $(\mathrm{TOH})$, tetrahexahedra $(\mathrm{THH})$ and hexoctahedra $(\mathrm{HOH})$. In FAOR studies, the order of activity at $0 \mathrm{~V}_{\mathrm{Ag} / \mathrm{AgCl}}$ followed: $\operatorname{cubes}_{(100)}<\operatorname{octahedra}_{(111)}<\mathrm{TOH}<\mathrm{HOH}<\mathrm{THH}_{(720)}<$ $\mathrm{THH}_{(210)}<\mathrm{THH}_{(520)}$. In that study, the activity was solely correlated to the facets presented by the NCs, rather than any electronic or strain effects brought about by the core-shell structure. ${ }^{250}$ Tripodal Pd-Cu alloy NCs were almost 8 times more active for the FAOR compared with Pdblack, which was mainly attributed to the exposed (211) facets, although in this case the surface $\mathrm{Cu}$ atoms were also suggested to facilitate FAOR oxidation, indicating that doping could hold unique advantages in catalyst design but again facet dependence seems to be more important. ${ }^{251}$ In one report by Sun et al., $\mathrm{Pd}-\mathrm{Cu}$ and $\mathrm{Pd}-\mathrm{Co}$ were both reported to be more active than $\mathrm{Pd}$, with $\mathrm{Pd}-\mathrm{Cu}$ being superior. ${ }^{252}$ The less electropositive $\mathrm{Cu}$ atoms in the surface were postulated to promote the adsorption and dehydrogenation of formic acid. ${ }^{252}$ However, in another study, Pd-Co alloys were found to be highly active for the FAOR, whilst Pd-Cu alloys were even less active than Pd alone. ${ }^{253}$ Clearly, further studies are required to ascertain the specific role of dopants in these reactions, which will enable more targeted synthesis. Wang et al. have combined specific structural and compositional catalyst design, synthesizing tetrahexahedral nanocages of $\mathrm{Pt}_{3} \mathrm{Ni}$ alloy that achieve high mass activities. ${ }^{254}$ The nanocage morphology maximizes the surface/volume ratio and also presents reactive high-energy facets, while the inclusion of $\mathrm{Ni}$ makes the catalyst more $\mathrm{CO}$-tolerant. Other work by Li et al. has displayed high 
control over NC catalyst composition and structure through complex alloying. For example, ternary Co-Pt-Au NCs form an ordered Co-Pt intermetallic core with a Pt-Au surface alloy, resulting in a non-CO FAOR pathway. ${ }^{255}$ The presence of $\mathrm{Au}$ in the Fe-Pt-Au ternary alloy causes a structure transformation during annealing at $600{ }^{\circ} \mathrm{C}$, from disordered $f c c$-Fe-Pt to ordered $f c t$-Fe-Pt-Au, which exhibits superior FAOR activity and stability (Figure 18C-E). The segregation of surface $\mathrm{Au}$ from the $f c t$-Fe-Pt core makes these catalysts particularly stable against CO-poisoning. ${ }^{256}$ Ternary alloys are generally well-suited to form intermetallic alloys, can tune $d$-band structures and tend to be more resistant to metal leaching during electrocatalysis. ${ }^{257,258}$

To summarize this section, the fine tunability of shapes and compositions accessible by colloidal chemistry has shown that the catalyst structure is crucially important in FAOR and dominates also over electronic effects in bimetallic catalysts. Specifically, for Pd-based catalysts, which are the most studied across the literature (Table S4), the presence of (100) and (211) facets results into the highest activities.

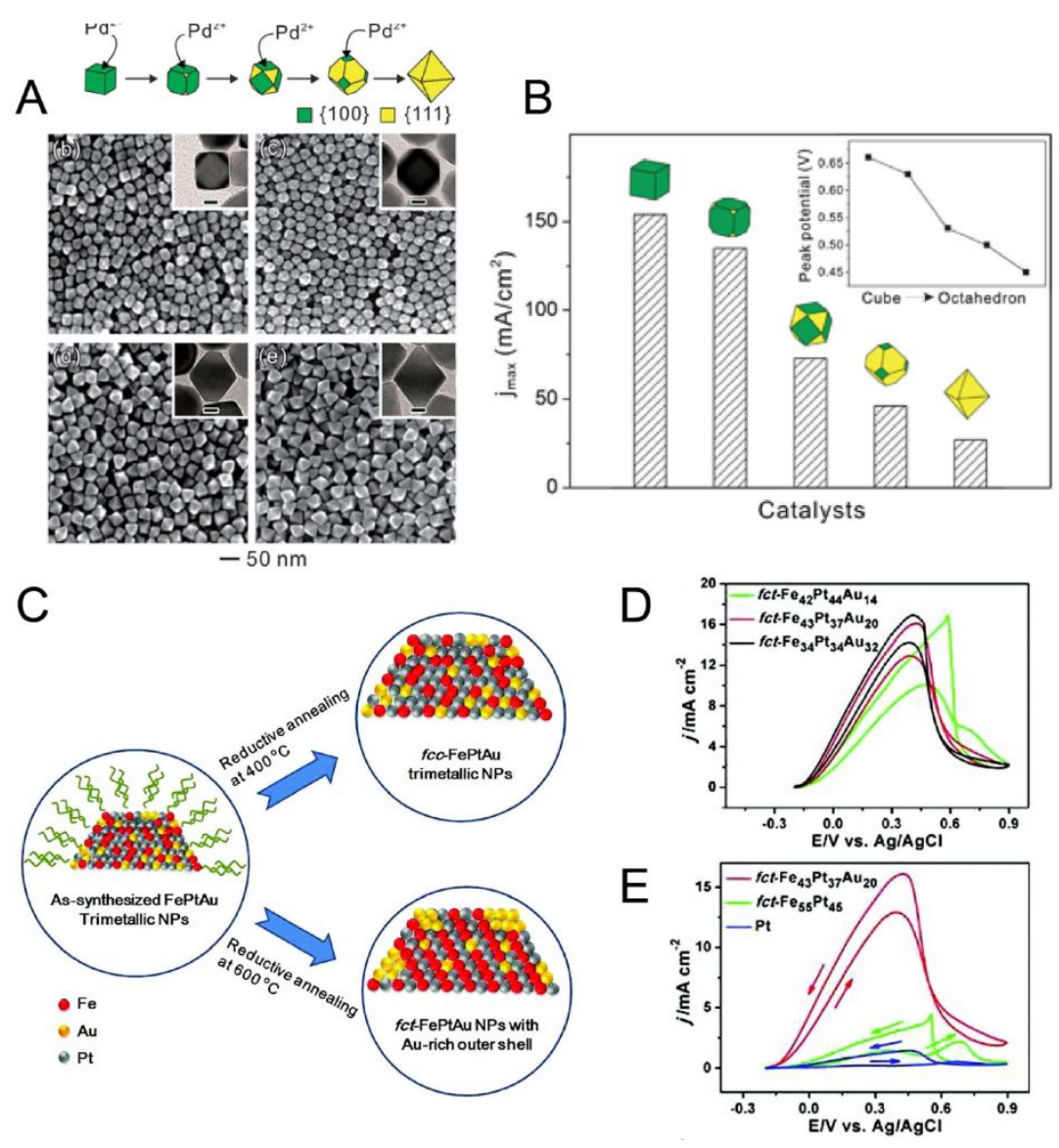

Figure 18. Pd NCs as electrocatalysts for FAOR. (A) Schematic illustrating the transformation of a Pd nanocube to an octahedron by continuous growth on the (100) planes, 
and SEM images of the polyhedra. (B) Maximum current densities in the FAOR over Pd NCs enclosed by different proportions of (100) and (111) facets; the inset plot shows the corresponding trend of the FAOR peak potential. Adapted with permission from reference 241, copyright 2012 Royal Society of Chemistry. (C) Schematic illustration of the structural change of Fe-Pt-Au NCs upon annealing, where the particles form a disordered $f c c$ structure at $400{ }^{\circ} \mathrm{C}$, whilst $\mathrm{Au}$ segregation is observed at $600{ }^{\circ} \mathrm{C}$, resulting in an $f c t$ structure. (D) Cyclic voltammograms showing the dependence of the FAOR activity on the $f c t$-Fe-Pt-Au composition. (E) Cyclic voltammograms highlighting the positive role of $\mathrm{Fe}$ and $\mathrm{Au}$ in the $f_{c t}$ Fe-Pt-Au ternary alloy. Adapted with permission from reference 256. Copyright 2012, American Chemical Society.

\subsection{Methanol oxidation.}

Pt is one of the most active single-metal catalysts in the MOR (Figure 19). ${ }^{259}$ However, one of the major challenges associated with the MOR is the propensity of $\mathrm{Pt}$ to be poisoned by the $\mathrm{CO}$ intermediate. The $\mathrm{pH}$ of the electrolyte plays an important role in influencing the facetdependence of the reaction on $\mathrm{Pt}$, where $\mathrm{Pt}(100)$ often excels in acidic media ${ }^{260}$ and $\mathrm{Pt}(111)$ in alkaline media. ${ }^{261}$ At the same time, the presence of low coordinated sites has been shown to activate the (111) Pt surface even in acid media by making it less susceptible to CO poisoning. ${ }^{262}$ The presence of additional metals can also weaken the Pt-CO binding strength and also increase the rate of $\mathrm{CO}$ oxidation; ${ }^{263}$ the extent of poisoning can therefore be reduced while the complete oxidation to $\mathrm{CO}_{2}$ can be promoted with alloy catalysts.

Combining structural elements with alloying has been the main focus of the studies across literature involving colloidally synthesized MOR catalysts (Table S5). Wires, rods, dendrites, tetrahedra and spheres account for many of the most promising systems as they maximize the exposure of (111) facet on the surface. ${ }^{264-275}$

For example, Yin et al. made use of tetrahedral and cubic Pd-Pt NCs in the MOR, showing that the (100) facets were more active, while the (111) facets were more durable, thus preferred for long term operations. ${ }^{272}$ Conversely, Huang et al. showed that Pt-Ru nanowires with (111) facets were both more durable and more active than (100)-capped cubes, showing that different compositions can alter the facet-dependence in the reaction. ${ }^{269}$ 

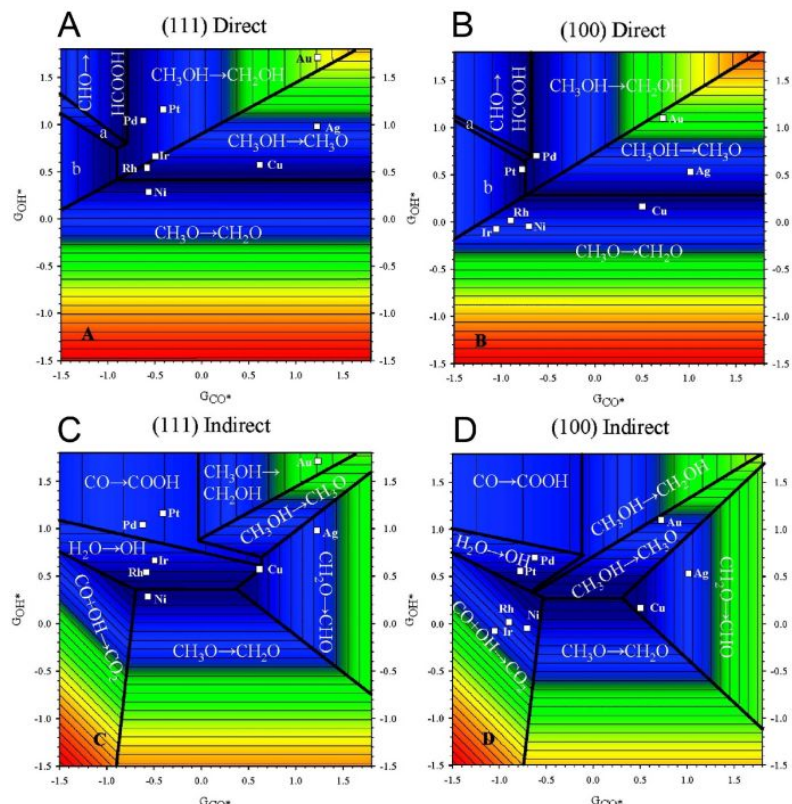

Low $\quad$ Onset Potential

High

Figure 19. Volcano plots for the MOR on (111) and (100) facets of different transition metals, for both $(\mathrm{A}, \mathrm{B})$ the indirect and $(\mathrm{C}, \mathrm{D})$ direct pathways. The adsorption free energies of $\mathrm{CO}^{*}$ $\left(G_{\mathrm{CO}^{*}}\right)$ and $\mathrm{OH}^{*}\left(G_{\mathrm{OH}^{*}}\right)$ are used as descriptors to determine the potential-limiting steps. The potential-limiting steps are written in each region. Adapted with permission from reference 259. Copyright 2009, American Chemical Society.

Several works have focused on combining the benefits of alloying and reactive high index facets, including Pt-Cu NWs ${ }^{264}$, Pt-Co NWs ${ }^{265}$ and excavated nanocubes ${ }^{276}$, Pt-Sn nanocubes ${ }^{277}$ and these NCs have generally shown superior performance compared to the spherical counterparts. Another useful approach that can be made with NCs is to maximize the catalyst surface active area using unique morphologies. ${ }^{271,278,279}$ For example, Gu et al. synthesized Pt$\mathrm{Ru}$ dendritic structures that showed improved activities over unshaped NCs of the same composition. ${ }^{271}$ Lie et al. developed Pd-P-B mesoporous spheres that exhibited much higher activities than Pd-P-B spherical NCs (Figure 20A,B). ${ }^{278}$

A few dedicated and systematic examples have also highlighted how subtle tuning of alloy compositions while keeping the same NC shape (i.e. dominant exposed facets) can greatly impact the MOR. For example, the tunable composition of tetrahedral core-shell $\mathrm{Cu} / \mathrm{Pt}-\mathrm{Ni} \mathrm{NCs}$ enabled exceptional MOR activity, where the role of Ni was attributed to increasing the amount of adsorbed $\mathrm{OH}$ that in turn promoting the oxidation of adsorbed $\mathrm{CO}$ on $\mathrm{Pt}$ sites (Figure 20C,D). ${ }^{280}$ However, higher amounts of Ni resulted in a decrease in the activity, which could be attributed to the decreased number of Pt sites. 
A
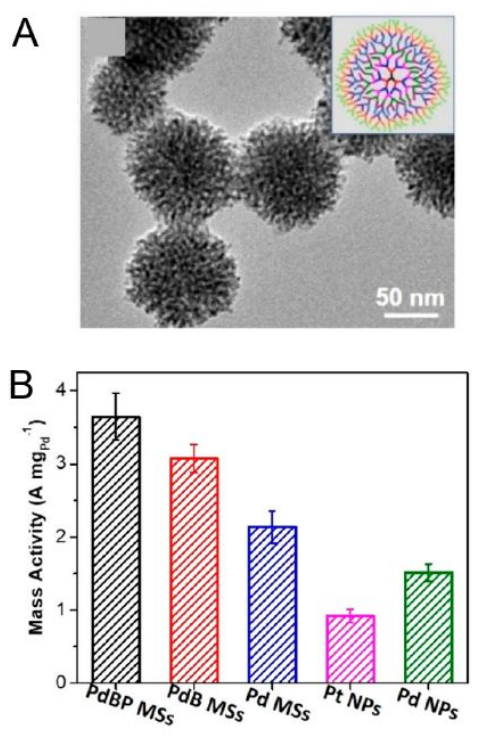

C
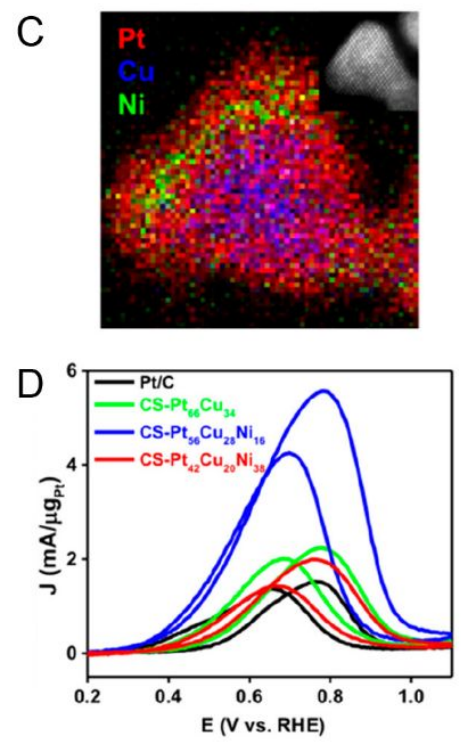

E
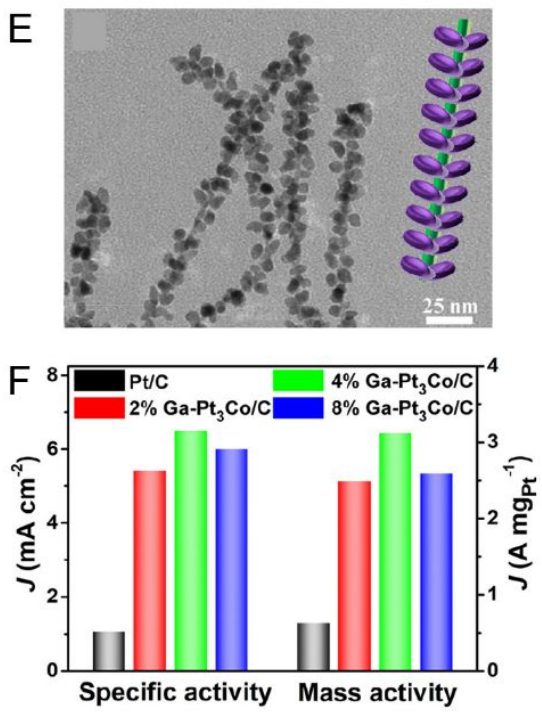

Figure 20. Colloidal NCs as electrocatalysts for MOR. (A) TEM image of Pd-P-B mesoporous nanospheres and (B) their MOR mass activities in comparison with related 
catalysts. Adapted with permission from reference 278. Copyright 2019, American Chemical Society. (C) EDS map of a Cu/Pt-Ni core-shell tetrahedron and (D) their MOR mass activities in comparison with related catalysts. Adapted with permission from reference 280. Copyright 2019, American Chemical Society. (E) TEM image of lavender-like dendritic NWs of Gadoped $\mathrm{Pt}_{3} \mathrm{Co}(4 \% \mathrm{Ga})$ and $(\mathrm{F})$ the MOR specific and mass activities in comparison with NWs of different Ga content. Adapted with permission from reference 268. Copyright 2020, American Chemical Society.

\subsection{Ethanol, ethylene glycol and glycerol oxidation.}

As for the MOR, one of the great challenges of the higher alcohol oxidation reactions is that $\mathrm{CO}$ is a possible reaction intermediate that can lead to catalyst deactivation. However, a unique challenge for $\mathrm{C}_{2+}$ alcohol oxidations is that the $\mathrm{C}-\mathrm{C}$ bond must be broken to achieve full oxidation. These two challenges taken together make the design of alcohol oxidation catalysts difficult, as the 100 facet is generally the best at cleaving the $\mathrm{C}-\mathrm{C}$ bond, yet is also the most prone to CO poisoning.

One way of approaching this issue is to make use of NC shapes bearing 111 facets, which are more stable, and then maximize the number of active sites through morphological control in order to achieve higher activities. In this theme we can see wires, tubes, cages, mesoporous spheres, sheets and dendritic structures that seem to yield improvements in activity in comparison with simple spheres. ${ }^{264,265,268,278,281-288}$

For example, $\mathrm{Pd}-\mathrm{Rh}-\mathrm{Te}$ nanotubes combine cooperative electronic effects in the ternary alloy with a hollow nanostructure that maximizes surface reaction sites. ${ }^{285}$ Zheng et al. synthesized $\mathrm{Pd}-\mathrm{Cu}$ nanosheets and demonstrated their benefits in the EOR, where large reductions in the overpotential and large increases in activity were observed, in comparison with Pd-Cu NPs and Pd nanosheets. ${ }^{289}$ Although nanosheets are a 2D NC morphology, these Pd-Cu nanosheets were described as having an overall 3D morphology due to wrinkling in the sheets. The improvement in their activity was attributed to the abundance of active sites, improved electrical conductivity and mass transport, and the synergistic effect between $\mathrm{Cu}$ and Pd. The Pd-P-B mesoporous nanospheres that were discussed in the MOR section also showed advantages in the EOR and GOR reactions. ${ }^{278}$

This approach is of course not limited to NCs bearing (111) facets. Xia et al. have also shown that Pd-Ru nanocages, which mainly present the more active (100) facets, are exceptional GOR and EGOR catalysts. ${ }^{290}$ The ultrathin, porous and hollow cage structure greatly increases the number of available surface sites, whilst the intrinsic activity increases due to the ligand effect 
of alloying $\mathrm{Cu}$ and $\mathrm{Pd}$. Whilst (100) facets are more prone to $\mathrm{CO}$ poisoning, the presence of $\mathrm{Ru}$ in the alloy mitigate this issue by lowering the $\mathrm{CO}$ adsorption energy.

In the case of higher alcohol oxidations, the presence of high-index facets is clearly advantageous, as the high surface energies can promote the breaking of the $\mathrm{C}-\mathrm{C}$ bond. For example, Han et al. made use of a series of $\mathrm{Au} @ \mathrm{Pd}$ NCs to illustrate the facet dependence of the EOR activity, where the activity increased in the order octahedra $(111)<$ cubes $(100)<$ hexoctahedral $(431)<$ convex polyhedral $\left(\begin{array}{llll}1 & 5 & 5 & 3\end{array}\right) .{ }^{291}$

Making use of the cooperative electronic effects of multiple metals in binary and tertiary NCs is also extremely common in alcohol oxidation catalysis. ${ }^{253,262,266,277-279,281,283-285,286,287,289-296}$ In the given examples, Pt and Pd have been alloyed with a wide selection of transition metals, and it could be said that additional metals are chosen to some extent by a phenomenological approach rather than judicious design at this point. That being said, there are a few examples that clearly demonstrate the benefit of fine-tuning catalyst composition. To highlight one example, Strasser et al. show how octahedral Rh-Pt-Sn catalysts improve in EOR performance as the $\mathrm{Rh}$ content is increased to a certain point, but after a full $\mathrm{Rh}$ shell is formed, the activity worsens as the cooperative electronic effect of all three metals is lost. ${ }^{293}$ In a second example, Pal et al. studied a series of Au-Pt-Pd NCs and showed that the ternary core-shell structures (i.e. Pd NPs and Pt NPs on Au nanorods) were far more active than the related $\mathrm{Au} @ \mathrm{Pt}$ or Au@Pd catalysts. Specifically, the Au@Pt@Pd catalyst was the most stable, where the formation of $\mathrm{PdO}$ helps to oxidize adsorbed $\mathrm{CO}$ and $\mathrm{CH}_{3} \mathrm{CHO}$ intermediates. Whilst $\mathrm{Au}$ in this case is situated at the core of the core-shell NC, Au-free catalysts did not perform nearly as well as the ternary NCs, indicating cooperativity between the metals. ${ }^{298}$

\section{Nanocrystal-based composites}

Combining NC catalysts with chemically diverse building blocks is another possible strategy to tackle the challenges of electrocatalytic reactions by decoupling some of the different limiting parameters discussed above. Embedding the catalyst in thin, porous and conductive skins can prevent morphological degradation of colloidal NCs, which is often observed under applied potentials. ${ }^{63,299}$ In addition, porous matrices can also positively affect mass-transport limitations and/or act as a hydrophobic layer on the catalyst surface and thus impact the product selectivity. ${ }^{300}$ Domains of different chemical nature may undergo synergistic interactions with 
the metallic NCs and thus modify their electronic structure, leading to improved catalytic activity and selectivity. ${ }^{53}$ Moreover, the bifunctionality in composite catalysts may help to stabilize specific reaction intermediates and thus break the linear scaling relationships present in transition metals. ${ }^{201,301,302}$ Indeed, different materials, including the surface ligands themselves, carbon-based supports, polymers and metal-organic frameworks (MOFs), have been shown to positively impact the catalytic environment of colloidal NCs and their performance for the electrochemical conversion of $\mathrm{CO}_{2}$, water, oxygen and hydrogen.

\subsection{Surface ligands as co-catalysts}

Surface ligands can be designed in a way that they do not impede catalysis, but rather improve it, either in terms of activity or selectivity or both. ${ }^{41,44}$ Ligand effects can be grouped into two categories: inner-sphere and outer-sphere effects. The former involves how the ligands influence the catalyst itself, including its electronic structure or its surface charge. The latter describes how they influence the local environment at the interface between the surface and the reaction medium.

Modification of the surface electronic structure via ligand binding has proven to be important in HER electrocatalysis. For example, Benson et al. demonstrated that the performance of ligand-decorated $\mathrm{MoS}_{2}$ nanosheets for HER was highly sensitive to the Hammett parameter (Figure 21A). ${ }^{303}$ Specifically, the most electron-donating ligand induced the lowest overpotentials, smallest Tafel slopes and charge-transfer resistances; the overpotential was regulated by $0.5 \mathrm{~V}$ just by tuning the ligands, thus demonstrating the huge potential of such approaches. As a second example, Tappan et al. recently made use of an NHC ligand to greatly improve the HER catalysis of $\mathrm{Cu}_{3-\mathrm{x}} \mathrm{P} \mathrm{NCs}$, where the strong electron donation from the ligand reduced the electrostatic repulsion between $\mathrm{Cu}^{+}$and $\mathrm{H}^{+} .{ }^{304}$ In another study, the HER activity of Ru NCs was demonstrated to increase in the presence of phenylpyridine ligands, in both acidic and basic media. ${ }^{305}$ This result was attributed to the moderate metal-ligand binding strength, which permitted a larger number of H-atoms to adsorb to the surface. A variety of inner-sphere effects have been observed in ORR electrocatalysis. To cite one example, Zhou et al. showed that ligands on very small Pt NCs $(2 \mathrm{~nm})$ can control the electronic structure of the metal surface and in turn, the activity towards the ORR (Figure 21B). ${ }^{306}$ Here, more electronegative ligands induce weaker binding of $* O$ intermediates, leading to higher activities. Alkyne ligands have also been suggested to modulate the electronic behavior of the surfaces of Au-Pd and Au-Ag NCs. ${ }^{307,308}$ After functionalization of Pt NCs with chlorophenyl ligands, the mass activity for the ORR was reported to increase by a factor of 2.8 , although the mechanism 
by which this occurs is unknown. ${ }^{309}$ Alba-Molina et al. showed that citrate surprisingly promotes both the ORR and HER activity of Au NCs, which was attributed to the small size of the ligand as well as the high overall negative charge-density at the surface, which promotes the binding of $\mathrm{O}_{2}$ and $\mathrm{H}_{3} \mathrm{O}^{+}$intermediates. ${ }^{310}$

In addition to inner-sphere effects, outer-sphere effects of ligands on ORR NC catalysts have also been observed. For example, Pt@Au NCs decorated with a perfluorosulfonic acid (PFSA) ligand show enhanced ORR activity. ${ }^{311}$ Much of the improvement in that case was attributed to the ligand and strain effects of the core-shell structure; however, the $\mathrm{SO}^{-}$groups from the acid were also suggested to participate in the transfer of reactive species. Mirkhalaf et al. showed that hydrophobic decylphenyl ligands on Au NCs promote the 2-electron reduction of $\mathrm{O}_{2}$ to $\mathrm{H}_{2} \mathrm{O}_{2}$, as the non-aqueous interface stabilizes superoxo and peroxo intermediates. ${ }^{312}$ Similar effects were proposed by Miyabayashi et al., where Pt NCs were functionalized by a mixture of OLAM and a pyrene-containing amine. ${ }^{313}$ The increased activity was attributed to ligand-induced changes in intermediate adsorption energies, as well as the non-polar ligand sphere that might increase local $\mathrm{O}_{2}$ concentration near the surface.

The use of ligands as promoters has been attracting much interest in the $\mathrm{CO}_{2} \mathrm{RR}$, especially with the aim of improving selectivity. Probably due to the intrinsic complexity of this reaction, most of the studies to date have been carried out on foil electrodes, which will also be included here for brief discussion. The modulation of electron density has been identified as a main inner-sphere effect of ligands on $\mathrm{CO}_{2} \mathrm{RR}$ catalysts, which impacts both the electron-transfer kinetics and the binding of intermediates. ${ }^{53,314-318}$ Cao et al. showed that NHC ligands (strong $\sigma$-donors) on $\mathrm{Au} \mathrm{NCs}$ were highly effective at improving the activity, selectivity and overpotential of the $\mathrm{CO}_{2} \mathrm{RR}$ (Figure 21C). ${ }^{53} \mathrm{Kim}$ et al. identified that cysteamine interactions with Ag NCs localize unpaired spin density at the surface that stabilizes the ${ }^{*} \mathrm{COOH}$ intermediate, thereby improving the intrinsic activity. ${ }^{318}$

Introducing more elaborate functional groups has proved to be a powerful approach to stabilize reaction intermediates. ${ }^{314}$ For example, the $\mathrm{N}-\mathrm{H}$ bonds of cysteamine have been shown to stabilize $\mathrm{CO}_{2}$ and related intermediates on the surface of $\mathrm{Ag}$ and $\mathrm{Au}$ NCs (Figure 21D). ${ }^{316,319}$ Pendant functional groups (i.e. those not binding to the surface) can also play a role; for example, acting as proton shuttles. For instance, Fang et al. showed that pendant bases can act in this way, encouraging the protonation of $* \mathrm{COOH}$ intermediates. ${ }^{320}$

In terms of outer-sphere ligand effects in the $\mathrm{CO}_{2} \mathrm{RR}$, Pankhurst et al. have recently used imidazolium ligands as promoters for the reduction of $\mathrm{CO}_{2}$ to $\mathrm{CO}$ on $\mathrm{Ag}$ NCs (Figure 21E and F). ${ }^{321}$ While electronic effects related to the ligand Hammett parameter were identified, 
the hydrocarbon tail of the ligand played a more important role in tuning the selectivity and activity. Specifically, intermediate lengths were the best, as their hydrophobicity suppresses the competing HER while imposing minimum kinetic penalties on the diffusion of reactants to the surface. Hydrophobicity has indeed emerged as a key parameter in the $\mathrm{CO}_{2} \mathrm{RR}$ in related work on $\mathrm{Cu}$ electrodes. ${ }^{322,323}$ Superhydrophobic surfaces can lead to triple-phase boundaries, increasing local $\mathrm{CO}_{2}$ mass transport. ${ }^{322}$ The hydrophobicity of the ligand coating also influences the water concentration profile at the surface, which alters the proton binding energetics of $* \mathrm{H}$ at $\mathrm{Cu}$ and the resulting product distribution. ${ }^{323}$

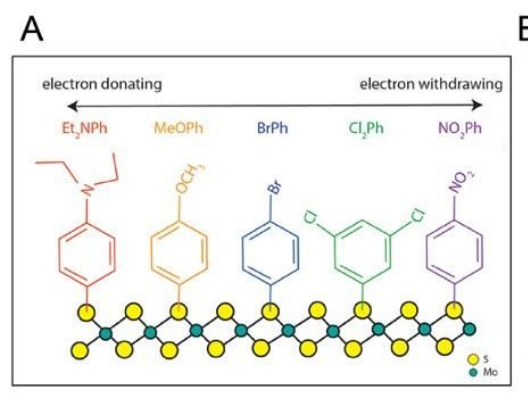

D

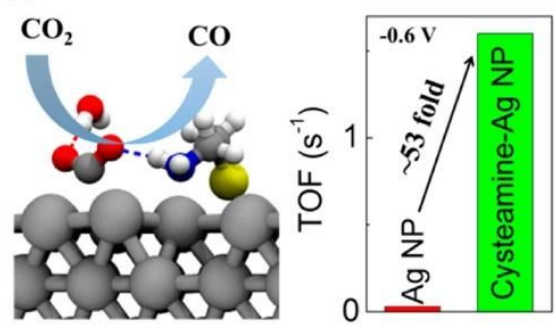

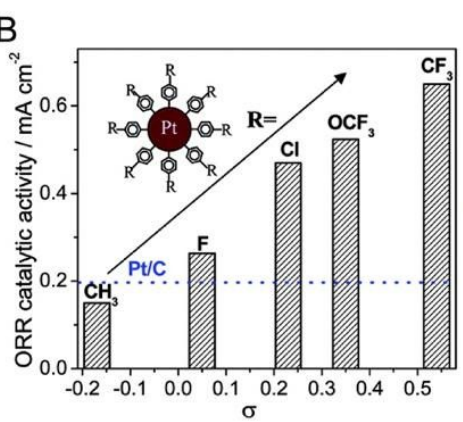

$\mathrm{E}$

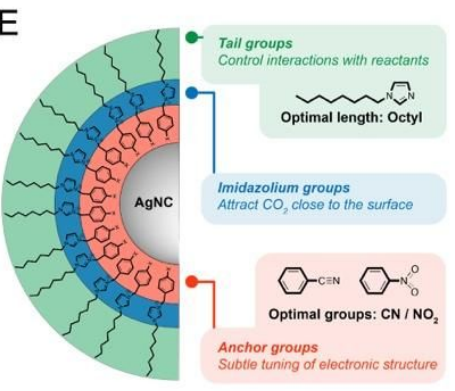

C

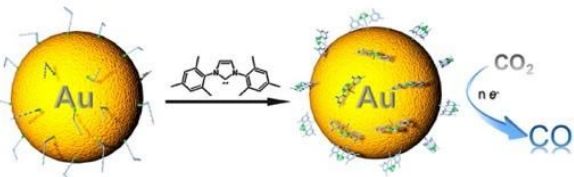

$\mathrm{F}$

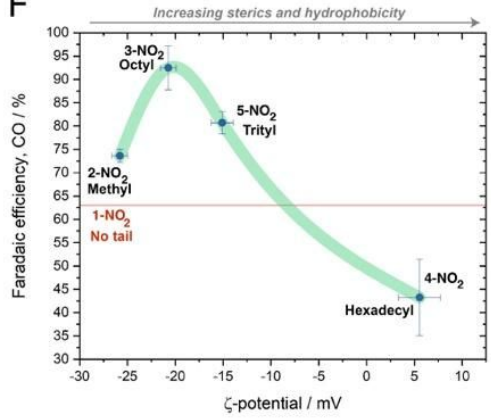

Figure 21. NC ligands as co-catalysts. (A) Ligand Hammett parameters tune the electronic structure of $\mathrm{MoS}_{2}$ nanosheets and regulate the activity and overpotential. Adapted with permission from reference 303. Copyright 2018, American Chemical Society. (B) Ligand Hammett parameters influence the ORR catalytic activity of Pt NCs. Adapted with permission from reference 306. Copyright 2012, American Chemical Society. (C) N-heterocyclic carbene ligands are strong $\sigma$-donors and improve the overpotential, activity and selectivity of Au NC $\mathrm{CO}_{2} \mathrm{RR}$ catalysts. Adapted with permission from reference 53. Copyright 2016, American Chemical Society. (D) Pendant $\mathrm{N}-\mathrm{H}$ groups in cysteamine ligands stabilize approaching $\mathrm{CO}_{2}$ molecules on the surface of $\mathrm{Ag} \mathrm{NC} \mathrm{CO} 2 \mathrm{RR}$ catalysts. Adapted with permission from reference 316. Copyright 2018, American Chemical Society. (E) Imidazolium ligands on Ag NCs improve $\mathrm{CO}_{2} \mathrm{RR}$ catalysis primarily by tuning the hydrophobicity of the $\mathrm{NC} /$ electrolyte interface. (F) Peak CO selectivity is achieved with Ag NC / imidazolium catalysts when the 
hydrocarbon tail of the ligand introduces hydrophobicity to the surface, while imposing minimum steric and kinetic penalties on the approaching reactants. Adapted with permission from reference 321 . Copyright 2019, Royal Society of Chemistry.

\subsection{Carbon-based materials}

Carbon-based materials used in $\mathrm{HER} / \mathrm{HOR}, \mathrm{OER} / \mathrm{ORR}$ and $\mathrm{CO}_{2} \mathrm{RR}$ include nanotubes, graphene and graphene oxide, carbon black $\left(\mathrm{C}_{\text {black }}\right)$ and carbon nitride. ${ }^{324,325}$ Their role spans from improving charge transport to inferring increased stability and to synergistically interacting with the $\mathrm{NC}$ catalysts to steer selectivity.

One common approach across the literature is to embed NCs in carbon materials to improve their stability. For example, Chung et al. developed a method to form an N-doped carbon shell in situ from a dopamine coating (Figure 22A,B). ${ }^{190}$ This shell prevented sintering of the ordered intermetallic FePt NCs which were then stable over a $100 \mathrm{~h}$ test in a membrane electrode assembly (MEA) fuel cell (Figure 22C). Similarly, reduced graphene oxide wrapped around $\mathrm{Cu} \mathrm{NWs}$ has been shown to block their clustering during $\mathrm{CO}_{2} \mathrm{RR}$, thus preserving morphology and product selectivity. ${ }^{222}$ In addition to improved stability, Rogers et al. have demonstrated that the overall electrocatalytic $\mathrm{CO}_{2} \mathrm{RR}$ performance of $8 \mathrm{~nm} \mathrm{Au}$ NCs increases when they are embedded in bottom-up synthesized graphene nanoribbons (GNRs) (Figure 22D and E). ${ }^{326}$ The catalytic environment created by the GNRs dramatically increased both the FE and the intrinsic activity toward $\mathrm{CO}$ in comparison to a $\mathrm{Au} / \mathrm{C}_{\text {black }}$ reference (Figure 22F). Electrodes prepared with $\mathrm{Au} / \mathrm{GNR}$ composites showed consistent catalytic performance during $24 \mathrm{~h}$ of electrolysis due to the effective immobilization of the NCs through strong dispersion interactions with the GNR matrix. A unique advantage to other graphitic support materials is that GNRs can be precisely tuned at the molecular level. Here, GNRs were functionalized with methyl carboxylates and a significantly higher reaction rate (Figure 23G) was observed for the resulting $\mathrm{Au} / \mathrm{GNR}$ catalyst indicating that the introduced ester groups interact with the $\mathrm{CO}_{2}$ molecule and change the reaction mechanism on the Au NC surface. 
A
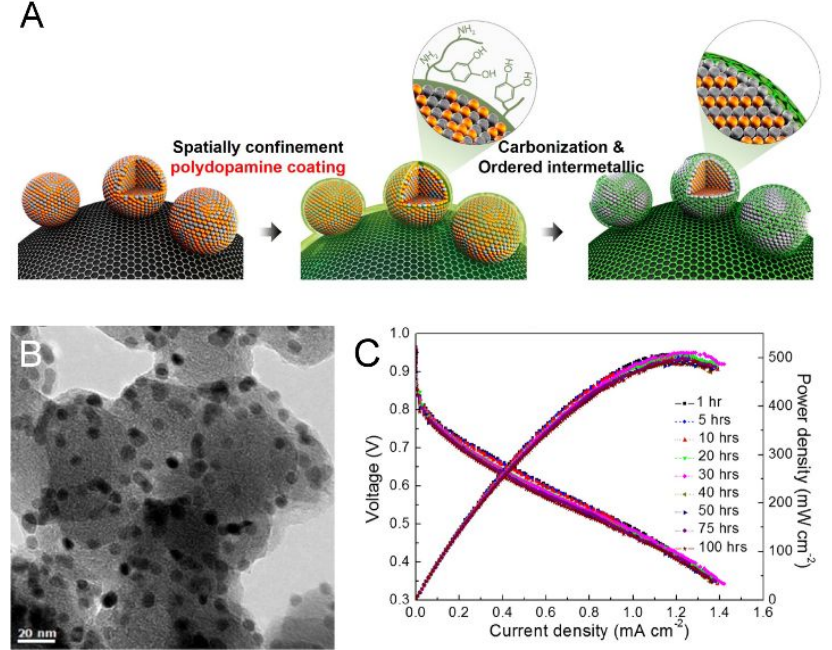

$\mathrm{D}$
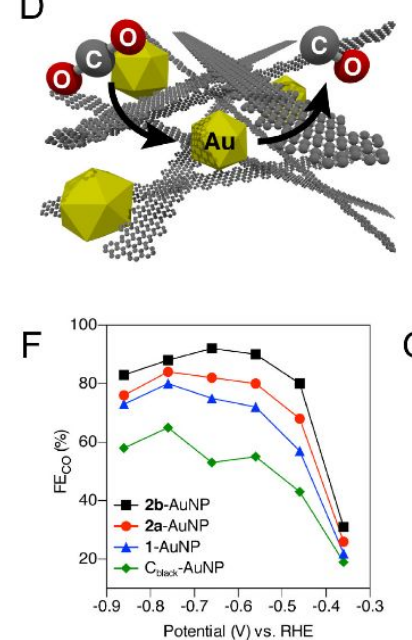

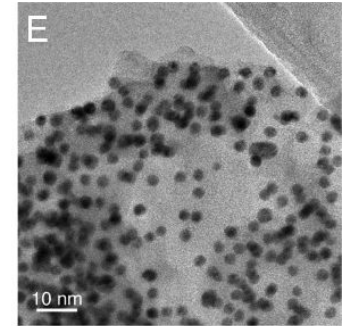

G

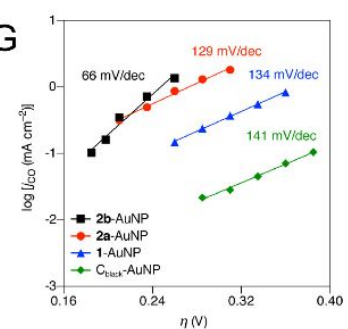

Figure 22. NC/carbon-materials composites as electrocatalysts. (A) Scheme illustrating the synthesis of carbon supported and N-doped carbon coated ordered PtFe NCs. (B) TEM image of dopamine coated NCs after annealing at $700{ }^{\circ} \mathrm{C}$. (C) Continuous operation for $100 \mathrm{~h}$ of the PtFe NCs catalyst in a membrane electrode assembly (MEA) revealing its long-term stability. Adapted with permission from reference 190. Copyright 2015, American Chemical Society. (D) Schematic illustration of $\mathrm{Au} / \mathrm{GNR}$ composites, which improve the stability of Au NCs and increase the $\mathrm{CO}_{2} \mathrm{RR}$ toward $\mathrm{CO}$. (E) TEM image of $\mathrm{Au} / \mathrm{GNR}$ composite. (F) FE towards $\mathrm{CO}$ for different $\mathrm{Au} / \mathrm{GNR}$ composites and the $\mathrm{Au} / \mathrm{C}_{\text {black }}$ reference measured at different potentials in $0.5 \mathrm{M} \mathrm{KHCO}_{3}$. $(\mathrm{G})$ Tafel slopes of the $\mathrm{CO}$ partial current density $\left(j_{\mathrm{CO}}\right)$ indicating that the functionalization of GNR (2b-AuNP) changed the $\mathrm{CO}_{2} \mathrm{RR}$ mechanism. Adapted with permission from reference 326. Copyright 2017, American Chemical Society.

Instead of embedding the as-synthesized NCs in a carbon matrix, they can also be directly synthesized on a broad variety of carbonaceous supports to further enhance the synergistic effects via strong metal-support interactions. ${ }^{327}$ For example, FePt NCs have been directly synthesized and assembled on graphene and demonstrated to be stable up to 10,000 cycles during ORR in acidic electrolyte, which is quite an impressive achievement for this material. ${ }^{328}$ Likewise, $\mathrm{Co}_{3} \mathrm{O}_{4}$ and $\mathrm{MnCo}_{2} \mathrm{O}_{4} \mathrm{NCs}$ prepared by direct nucleation and growth on graphene oxide were also shown to possess increased stability in alkaline conditions. ${ }^{329,330}$ Huang et al. have synthesized a composite catalyst comprising partially oxidized Co NCs $(5 \mathrm{~nm})$ dispersed on single-layer nitrogen-doped graphene (SL-NG), which showed highly selective $\mathrm{CO}_{2} \mathrm{RR}$ into $\mathrm{CH}_{3} \mathrm{OH}$ (FE of $71.4 \%$ at $-0.9 \mathrm{~V}_{\mathrm{SCE}}$ ) with decent stabilities up to $10 \mathrm{~h} .{ }^{331}$ The authors speculate synergistic interaction promoting the multiple proton-electron transfer steps involved in the reaction mechanism toward $\mathrm{CH}_{3} \mathrm{OH}$. 


\subsection{Polymers}

Polymeric binders, such as Nafion, are very often used to stabilize and disperse NCs in catalyst inks for deposition onto electrodes. Normally, it is assumed that this polymer does not interfere with the catalytic activity. However, a recent study by Lee et al. has focused on understanding the role of polymeric binders in $\mathrm{CO}_{2} \mathrm{RR}$ and highlighted that some effects are possible. ${ }^{333}$ The authors prepared working electrodes by mixing Au NCs $(5.5 \mathrm{~nm})$ on $\mathrm{C}_{\text {black }}$ with five different polymeric binders, namely Nafion, polyvinyl alcohol (PVA), polyacrylic acid (PAA), polyvinylidene difluoride (PVDF) and polytetrafluoroethylene (PTFE). The resulting catalyst inks selectively produced $\mathrm{CO}$ with the highest FE of $94.7 \%$ for the Au/PTFE composite at $-0.7 \mathrm{~V}_{\text {RHE}}$. In general, the F-containing binders (including Nafion) showed a higher $\mathrm{CO}$ selectivity, which was attributed to the weakening of the proton adsorption $\left({ }^{*} \mathrm{H}\right)$ on the $\mathrm{Au}$ surface and concomitant suppression of the HER.

Thus, polymers can be utilized or properly engineered in order to improve the catalytic performance. In one example, colloidally synthesized $\mathrm{Cu}$ NWs (diameter $\sim 100 \mathrm{~nm}$ ) were wrapped with polydopamine (PDA) and tested for the $\mathrm{CO}_{2} \mathrm{RR}$ by Liu et al. ${ }^{334}$ Similarly as for the graphene oxide-wrapped NWs discussed above, ${ }^{222}$ the PDA shell strongly enhanced the morphological and catalytic stability of the Cu NWs. Moreover, the $\mathrm{Cu} @$ PDA hybrid catalysts showed a more than double increase in the selectivity for methane compared to pristine $\mathrm{Cu}$ $\mathrm{NWs}$, which was attributed to the synergistic interaction of the $-\mathrm{NH}_{2}$ and $-\mathrm{OH}$ groups in PDA at the intimate contact with the $\mathrm{Cu}$ surface. The authors suggested that the $-\mathrm{NH}_{2}$ groups promote proton capture and delivery to the active sites where the $-\mathrm{OH}$ groups stabilize the $\mathrm{CO}^{*}$ intermediate, allowing for further protonation and $\mathrm{CH}_{4}$ formation. 
In a second example, Zhang et al. have investigated the effect of modifying metal nanocatalysts for $\mathrm{CO}_{2} \mathrm{RR}$ with polymeric N-heterocyclic carbenes (NHC) (Figure 23). ${ }^{335} \mathrm{Au}$ and Pd NCs were capped with two different polymeric NHCs (polydentate (P1) and monodentate (P2)) through a ligand exchange approach. The electrocatalytic performance of the composite catalysts were compared to other traditional ligands as thiol-terminated polystyrene $\left(\mathrm{PS}_{50}-\mathrm{SH}\right)$, oleylamine (OLAM) and 1-dodecanethiol (DDT) and revealed substantial improvements in activity and selectivity. Both polymers were shown to prevent the nanoclustering of the metal NCs under reductive potentials and thus improve their long-term stability. The increased selectivity toward CO for both Au NCs (FE 90\%) and Pd NCs (FE 65\%) was attributed to the strong $\sigma$-donation of the NHC polymers to the metal surface. Moreover, the polymer chains form a hydrophobic passive layer, which suppresses proton reduction and promotes $\mathrm{CO}_{2} \mathrm{RR}$. Recent studies have shown that $\mathrm{N}$-aryl pyridinium compounds in the electrolyte reductively dimerize or polymerize in situ, forming a layer of substituted tetrahydrobipyridines on $\mathrm{Cu} .{ }^{336-}$ 338 These dimeric or oligomeric hydropyridines act as promoters for the conversion of $\mathrm{CO}_{2}$ to $\mathrm{C}_{2} \mathrm{H}_{4}$. It was discovered that the electronic properties of the organic film alter the preferred binding modes of ${ }^{*} \mathrm{CO}$ intermediates on $\mathrm{Cu}$; peak $\mathrm{C}_{2} \mathrm{H}_{4}$ selectivity was achieved at optimal ratios of $\mathrm{CO}_{\text {bridge }}$ to $\mathrm{CO}_{\text {atop }}$ binding modes. ${ }^{338}$

Polymeric ligands have also been used to improve HER activities by regulating the local proton concentration. For example, work by Xu et al. demonstrated that poly(allylamine) on tripodal Pt NCs improves their HER activity as the pendant amino groups in the polymer behave as proton relays between the solvent and the surface, where the ammonium $\mathrm{RNH}_{3}{ }^{+}$cations increase the surface proton concentration. 339 


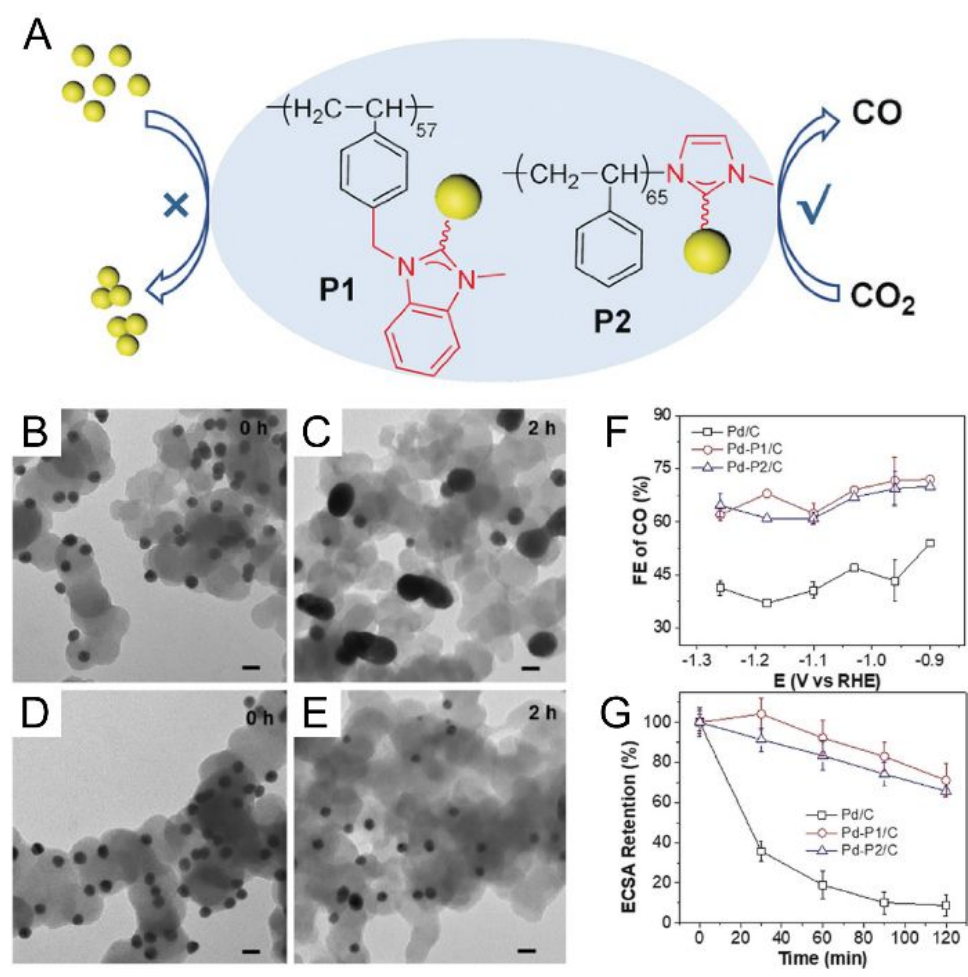

Figure 23. NC/polymers composites as electrocatalysts. (A) Schematic illustration of $\mathrm{NC}$ /polymeric NHC composites, which prevent $\mathrm{NC}$ clustering and increase the $\mathrm{CO}_{2} \mathrm{RR}$ selectivity toward CO. TEM images of $(B, C)$ unmodified Au NCs and (D,E) Au/polymeric $\mathrm{NHC}$ composites on $\mathrm{C}_{\text {black }}$ before (left) and after (right) $\mathrm{CO}_{2} \mathrm{RR}$ at $-0.9 \mathrm{~V}_{\mathrm{RHE}}$ in $0.1 \mathrm{M} \mathrm{KHCO}_{3}$ for $2 \mathrm{~h}$. (All scale bars are $20 \mathrm{~nm}$ ). (F) CO FE and (G) electrochemically active surface area (ECSA) retention over time (at $-1.26 \mathrm{~V}_{\mathrm{RHE}}$ ) of unmodified $\mathrm{Pd} \mathrm{NCs}(\mathrm{Pd} / \mathrm{C})$ and $\mathrm{Pd} /$ polymeric NHC composites (Pd-P1/C, Pd-P2/C). Adapted with permission from reference 335. Copyright 2019, Wiley.

\subsection{Metal-organic frameworks}

A significant number of studies on NC/MOF hybrids have demonstrated their promising synergistic effects to enhance a variety of organic and photocatalytic reactions. ${ }^{340}$

During recent years, a few attempts have been made to combine nanostructured metals with porous MOFs as electrocatalysts for the $\mathrm{CO}_{2} \mathrm{RR}$ in the search of strategies to steer the selectivity, one of the biggest challenges for this reaction. Kung et al. electrochemically reduced $\mathrm{Cu}(\mathrm{II})$ into a thin film of $\mathrm{NU}-1000$ to generate small metallic $\mathrm{Cu}$ NCs inside the MOF pores. ${ }^{341}$ Despite $\mathrm{Cu}$ NCs below $10 \mathrm{~nm}$ are mostly selective for hydrogen, the $\mathrm{Cu} @ \mathrm{NU}-1000$ hybrids showed electrocatalytic activity for $\mathrm{CO}_{2} \mathrm{RR}$ with a maximum $\mathrm{FE}$ of $28 \%$ for formate (at $\left.-0.82 \mathrm{~V}_{\mathrm{RHE}}\right)$. Guntern et al. functionalized colloidal Ag NCs $(10.5 \mathrm{~nm})$ with a thin shell of $\mathrm{Al}_{2} \mathrm{O}_{3}$ by atomic layer deposition (ALD), which then served as a localized precursor for the 
subsequent synthesis of Al-PMOF (Figure 24). ${ }^{342}$ The Ag@Al-PMOF hybrids showed a more than double increase in the selectivity toward $\mathrm{CO}\left(55.8 \% \mathrm{FE}\right.$ at $\left.-1.1 \mathrm{~V}_{\mathrm{RHE}}\right)$ and a drastic decrease of the HER compared to the bare Ag NCs. Similarly to the carbon nitride and NHC polymer composites described above, this enhanced $\mathrm{CO}_{2} \mathrm{RR}$ selectivity was mostly attributed to electron donation from the MOF to the NCs across a pristine interface between the two materials. A minor contribution of mass-transport effects was detected in particular at higher potentials. Furthermore, the pronounced sintering of the bare $\mathrm{Ag} \mathrm{NCs}$ during $\mathrm{CO}_{2}$ electrolysis was strongly inhibited when they are embedded in the MOF matrix. The same synthetic approach was extended to form hybrids with $\mathrm{Cu}$ and $\mathrm{Au} \mathrm{NCs}$, thereby revealing a new tool for the preparation of composite catalysts for the $\mathrm{CO}_{2} \mathrm{RR}$. In a recent study, Heidary et al. investigated a hybrid metal-organic catalysts comprising a mesoporous film of $20-30 \mathrm{~nm} \mathrm{TiO}_{2}$ NCs which were coated with a 2-3 nm shell of an electrocatalytic Mn-porphyrin containing MOF. ${ }^{343}$ The authors observed a fast electrochemical reduction and slow oxidation of the Mnporphyrin together with a reversible restructuring of the MOF. Moreover, they were able to detect the $\mathrm{CO}_{2}$ reduction intermediates for the first time in a MOF electrocatalyst and used DFT modeling to propose a mechanism for the formation of the main product $\mathrm{CO}$. By replacing the $\mathrm{Mn}$-porphyrin with $\mathrm{Fe}$ - and $\mathrm{Co}$-porphyrins, the turnover frequencies of $\mathrm{CO}$ and $\mathrm{CH}_{4}$ formation were increased up to 5- and 10-fold, respectively.
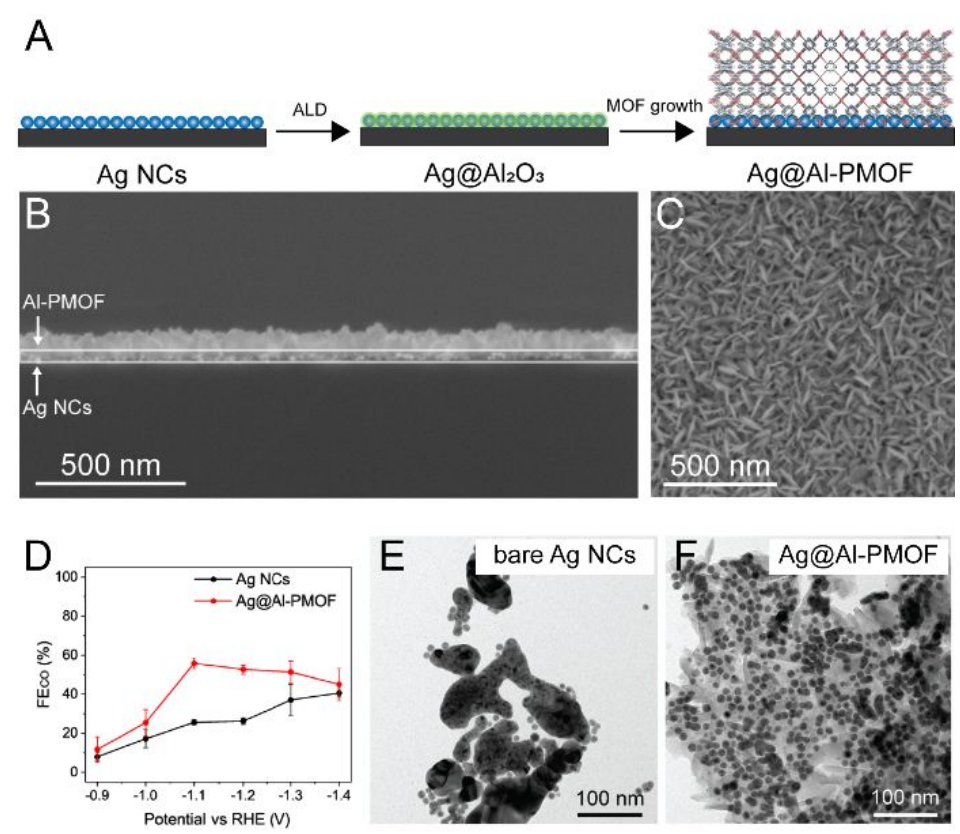

Figure 24. NC/MOF composites as electrocatalysts (A) Scheme illustrating the synthesis of Ag@Al-PMOF hybrids. (B) Cross-section SEM and (C) top-down SEM image of Ag@AlPMOF thin films. (D) CO FE for Ag NCs (black) and Ag@Al-PMOF hybrids (red) measured 
at different potentials in $0.1 \mathrm{M} \mathrm{KHCO}_{3}$. TEM images after $75 \mathrm{~min}$ of $\mathrm{CO}_{2}$ electrolysis of (E) bare Ag NCs measured and (F) Ag@Al-PMOF. Adapted with permission from reference 342. Copyright 2019, Wiley.

\section{Colloidal NC catalysts to understand reconstruction processes}

Well-defined size and shape of NC catalysts, where one particle looks like the other serve as a good model to study catalyst reconstruction. Electrocatalyst activity and stability often go one against the other and it is a matter of finding an acceptable compromise between the two metrics in the quest to identify the most suitable electrocatalysts. ${ }^{344}$ The control over nanoparticle shape and hence the surface crystallographic facet has contributed to identify the optimal Pt catalyst for HER, balancing its stability and mass activity ${ }^{163}$ and increase of the stability of Pt catalyst by favorable catalyst-support interactions. ${ }^{127}$ Similar principles are exploited in the search of a reasonably stable earth abundant element catalyst for HER, ${ }^{119,126,132}$ The non-noble metal leaching during operation was identified, eventually leading to a new class of dealloyed Pt-Ni and Pt-Co NCs with greatly improved mass activity stability in ORR compared to monometallic Pt NCs. ${ }^{45,169,179,182,183,186,187,345}$ From a library of alloyed ORR catalyst, it was identified, that $\mathrm{NC}$ surface distortions can rationalize activity in ORR as surface reconstruction inevitably occurs under simulated PEMFC cathode operation. ${ }^{346}$ The principle of dealloyed catalyst was successfully transferred to a Pt-Sn octahedral catalyst for EOR. ${ }^{286}$

In general, identical location TEM can provide detailed information on a single particle level, if the supporting electrode is suitable for both electrochemical testing and TEM imaging (i.e. electron transparent substrate). Stability of hollow Pt nanocatalyst was investigated with this technique at a single particle level. The nanoporosity was found to be metastable and subject to reorganization. Rate of the degradation processes was strongly dependent on the upper potential limit during the accelerated stress testing. ${ }^{347}$ Steps and corners of Pt-Ni ORR catalyst were identified as preffered sites for dissolution and interestingly, smaller particles were preffered for redeposition, contrary to conventional understanding of Ostwald ripening. ${ }^{348}$ High activity topologically complex NiPt alloy nanoparticles are especially susceptible to morphological evolution by electrochemically enhanced surface diffusion leading to coarsening and decrease in ECSA. It was demonstrated that decorating the catalyst surface with Ir, leads to pinning of surface steps, preventing their movement and limits the coarsening of 
the catalyst and stabilizes the nanoporous morphology (Figure 25A) ${ }^{345}$ Alternatively, the electron transparent electrode requirement can be circumvented with the use of identical location SEM, though this implies compromising the imaging resolution. 349

A

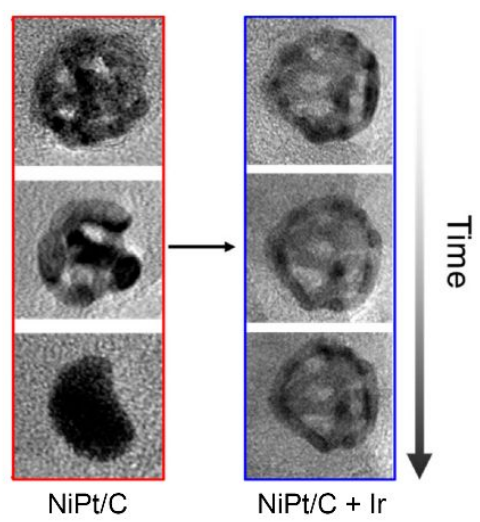

B
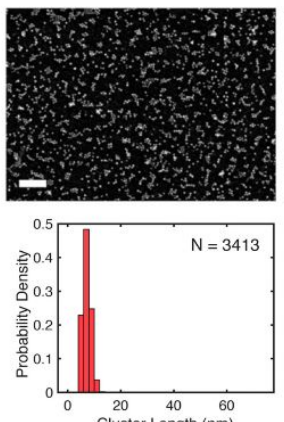

Cluster Length $(\mathrm{nm})$

$0 \mathrm{~min}$

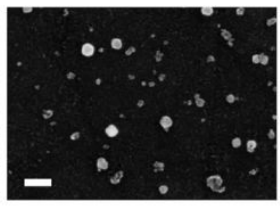

$\mathrm{N}=3222$

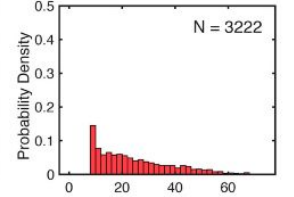

Cluster Length (nm)

$5 \mathrm{~min}$
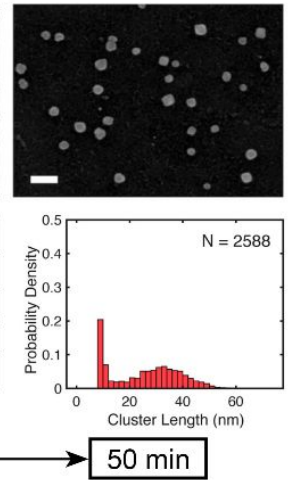

C
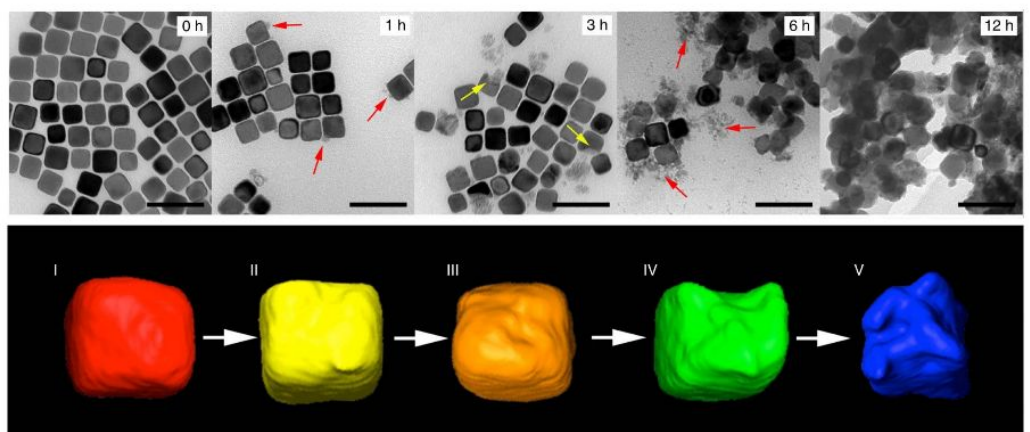

Figure 25. (A) Identical location TEM of dealloyed nanoporous NiPt and stabilized NiPt + Ir NCs during accelerated stress testing. Adapted with permission from reference ${ }^{345}$. Copyright 2017, American Chemical Society. (B) SEM documenting evolution of initially $7 \mathrm{~nm} \mathrm{Cu}$ spheres during $\mathrm{CO}_{2} \mathrm{RR}$ with corresponding particle size distributions. Adapted with permission from reference 350. Copyright 2019, American Chemical Society. (C) TEM images showing morphological evolution of initially well-defined $41 \mathrm{~nm} \mathrm{Cu}$ nanocubes during $\mathrm{CO}_{2} \mathrm{RR}$. Below are TEM tomography reconstructions of the $\mathrm{Cu}$ nanocubes at the corresponding stages. Adapted with permission from reference 63. Copyright 2018, Nature.

The interpretation of catalyst changes becomes difficult when spontaneous surface oxidation obstructs the fine changes on catalyst surface, which is the case of $\mathrm{Cu}$ in $\mathrm{CO}_{2} \mathrm{RR} .{ }^{351}$ Yet, good control over the initial size and shape of the catalyst still enables the interpretation of general trends in the catalyst changes. Small $\mathrm{Cu}$ nanospheres $(<15 \mathrm{~nm})$ are especially susceptible to reconstruction. ${ }^{217,221}$ The catalyst morphology evolves during $\mathrm{CO}_{2} \mathrm{RR}$ and, if the initial loading is high, leads to activation of the catalyst for $\mathrm{C}-\mathrm{C}$ coupling and production of ethylene. ${ }^{221}$ Identification of the active form of the catalyst was obscured by its susceptibility to rapid 
spontaneous oxidation, yet passivating the metallic $\mathrm{Cu}$ immediately after its activation lead to the identification of closely packed small $(<5 \mathrm{~nm}) \mathrm{Cu}$ crystallites, which are the active catalyst selective for ethylene. ${ }^{352}$ With careful statistical analysis of particle size distribution on the electrode, two separate reconstruction phenomena were identified. Initial sintering within the first 5 min leads to catalyst activation and fragmentation of the catalyst in later stages is connected to its deactivation (Figure 25B). ${ }^{350,352}$ These phenomena seem to have wider implications in $\mathrm{Cu}$ based catalysts for $\mathrm{CO}_{2} \mathrm{RR}$. Similar activation process was observed, when the initial catalyst consisted of $\mathrm{Cu}_{2} \mathrm{O}$ cubes, ${ }^{353}$ and loss of selectivity for ethylene production was connected to fragmentation of initially well-defined $\mathrm{Cu}$ cube shaped catalyst (Figure 25C) ${ }^{63,352}$ In this study, electron tomography revealed that fragmentation starts at the interface of the (100) facets and (110) edges, which was identified as the active sites in favoring C-C coupling. ${ }^{220}$ This finding is thus in line with the more active sites being also the faster degrading ones.

While the examples above highlight how ex-situ techniques combined with well-defined NCs helps to elucidate reconstruction processes, the catalytic sites are often generated electrochemically and only exist under reaction conditions. Therefore characterization of the catalyst before and after operation should be complemented with operando/in-situ measurements, if the goal is to understand these processes in full detail. 


\section{In situ and operando characterization}

The development of in situ and operando tools to study catalysts under relevant working conditions (i.e. high temperatures, high pressures, flow chemistry, applied potential) has enabled significant progress towards understanding the underlying chemistry of catalytic processes at the atomistic level and towards the establishment of relationships between the often evolving structure of a catalyst material and its corresponding catalytic performance. In fact, in situ and operando tools can both identify transient (i.e. short-living) intermediates otherwise undetectable by using common ex situ experiments. However, their exact definition is often at the center of debate in the community. This review refers to in situ for measurements performed under reaction conditions that closely resemble those of the bulk electrolysis cells and operando for those measurements performed in bulk electrolysis cells and thus allowing for simultaneous measurement of the catalytic activity. Nowadays, the most wellestablished tools for catalyst characterization under in situ or operando conditions are based on high-energy synchrotron radiation. Simultaneously, increasing efforts are dedicated to in situ and operando studies using electron microscopy, those being challenged by the downsizing of the electrochemical cell to fit in a microchip. There are numerous insightful examples employing in situ or operando tools for the characterization of $\mathrm{NC}$ catalysts for $\mathrm{CO}_{2} \mathrm{RR}, \mathrm{HER}$ or OER and we refer to comprehensive reviews already published on this topic. ${ }^{62,225,354-367,367-}$ ${ }^{369}$ In the following sections, our aim is to provide a simple description of the techniques and cells along with a few specific examples related to colloidally prepared NC catalysts.

\section{1. $X$-ray absorption and scattering}

Among different X-ray based tools, X-ray scattering and X-ray spectroscopy techniques are widely used due to their complementarity and non-destructive character. Briefly, X-ray scattering can be divided into elastic scattering (e.g. X-ray diffraction, XRD), where the wavelength and energy of the scattered wave do not change, and inelastic scattering (e.g. resonant/non-resonant Raman scattering - information on the electronic structures of the catalysts). On the other hand, X-ray absorption/emission spectroscopy (XAS and XES, respectively) rely on soft (below ca. $10 \mathrm{keV}$ ) and hard (from 10 to $120 \mathrm{keV}$ ) X-rays. Table 2 summarizes the most important scattering and spectroscopic techniques for in situ and operando studies in catalysis, including colloidal NC catalysts, along with the main information that can be extracted from them. 
Table 2. Summary of the X-ray spectroscopic and scattering tools employed to study NC catalysts.*

\begin{tabular}{|c|c|c|c|}
\hline Type & Technique & Key information extracted & Other observations \\
\hline \multirow{3}{*}{$\begin{array}{c}\text { X-ray } \\
\text { spectroscopy }\end{array}$} & $\begin{array}{l}\text { X-ray photoelectron } \\
\text { spectroscopy (XPS) }\end{array}$ & $\begin{array}{c}\text { Elemental composition and } \\
\text { oxidation state }\end{array}$ & Surface sensitive \\
\hline & $\begin{array}{c}\text { X-ray absorption spectroscopy } \\
\text { (XAS) }\end{array}$ & $\begin{array}{c}\text { Oxidation } \\
\text { state/coordination } \\
\text { environment }\end{array}$ & $\begin{array}{l}\text { Info on unoccupied } \\
\text { density of states }\end{array}$ \\
\hline & $\begin{array}{c}\text { X-ray emission spectroscopy } \\
\text { (XES) }\end{array}$ & $\begin{array}{c}\text { Electronic structure and } \\
\text { ligand environment }\end{array}$ & $\begin{array}{c}\text { Info on partially occupied } \\
\text { density of states }\end{array}$ \\
\hline \multirow{3}{*}{$\begin{array}{c}\text { X-ray } \\
\text { scattering }\end{array}$} & $\begin{array}{c}\text { Small-angle X-ray scattering } \\
\text { (SAXS) }\end{array}$ & Particles size and/or shape & Assembly periodicity \\
\hline & $\begin{array}{c}\text { Wide-angle X-ray scattering } \\
\text { (WAXS)/XRD }\end{array}$ & $\begin{array}{c}\text { Crystal structure and phase } \\
\text { identification }\end{array}$ & \\
\hline & $\begin{array}{l}\text { Pair distribution function - } \\
\text { total scattering (PDF) }\end{array}$ & $\begin{array}{c}\text { Local structure } \\
\text { determination in NCs }\end{array}$ & $\begin{array}{l}\text { Suitable for crystalline } \\
\text { and amorphous materials }\end{array}$ \\
\hline
\end{tabular}

* To note that XAS includes XANES (X-ray absorption near edge structure) and EXAFS (extended X-ray absorption fine structure).

Eventually, a combination of all these experiments is needed to monitor simultaneously the structural and the chemical speciation changes occurring at the solid/liquid (electrode/electrolyte) interface during electrocatalysis. For example, the combination of XRD (long-range order; crystalline materials) and XAS (short-range order; crystalline/amorphous materials) provides beautiful insights into the dynamic nature of a catalyst lifetime i.e. reconstruction and/or reconfiguration, poisoning and death. ${ }^{370}$ Vibrational spectroscopies are then highly useful to study the surface adsorbed molecules thus providing a complementary mechanistic investigation. ${ }^{300,371,372}$ It must be said that XRD and vibrational spectroscopies require rather complex setups (i.e. thin-layer design or the droplet geometry/free-hanging meniscus in the XRD case) that, together with the low catalyst loading, provide low current densities, making it challenging to have simultaneous product detection. ${ }^{370,373-377}$ Furthermore, the broad peaks associated with the NCs represent an additional complication for XRD studies. Therefore, XAS is definitely the "go-to" technique for many researchers in the field to gain electronic and structural information about NC catalysts.

Most of the XAS setups reported in the literature for in situ and operando studies during electrocatalysis are based on a fluorescence geometry (a one-compartment cell for HER/OER, and a batch or a two-compartment sandwich-type cell with a proton conducting membrane for 
$\mathrm{CO}_{2} \mathrm{RR}$ ) with the beam penetrating through the back-side via an X-ray transparent window made of silicon nitride $\left(\mathrm{Si}_{3} \mathrm{~N}_{4}\right)$, amorphous carbon or Kapton ${ }^{\circledR}$ foil. ${ }^{363-368}$ However, there are also examples of XAS electrochemical experiments performed using the more traditional transmission geometry, ${ }^{378}$ where the thickness of the electrolyte layer becomes a limiting factor. Conductive layers such as ITO (indium-doped tin oxide), FTO (fluorine-doped tin oxide), carbon cloth, or Au are commonly used as working electrodes (WE). The reference electrode (RE) is placed close to the working electrode, and the counter electrode (CE) is positioned further away. On the other hand, in the soft X-rays cells, typically a SiN/C membrane is employed to separate the cell environment from the UHV needed for the soft Xrays measurements. 379,380

Figure 26 reports two representative examples of operando XANES and EXAFS for colloidal NCs utilized as $\mathrm{CO}_{2} \mathrm{RR}$ electrocatalysts. In the first example a two-compartment cell (Figure 26A) was utilized to monitor the oxidation state of $\mathrm{Cu} / \mathrm{CeO}_{2}$ heterodimers which were found to promote $\mathrm{CO}_{2} \mathrm{RR}$ versus $\mathrm{HER}$ and to be highly selective towards the production of methane. ${ }^{354}$ XANES measurements highlighted that a partial reduction from $\mathrm{Ce}^{4+}$ to $\mathrm{Ce}^{3+}$ takes place under $\mathrm{CO}_{2} \mathrm{RR}$ conditions (Figure 26B) accompanied by the formation of oxygen vacancies which was demonstrated by other complementary techniques (XPS, Raman, UVVis). Thanks to these data, DFT calculations could assess the important role of such oxygen vacancies in the stabilization of the $\mathrm{CHO}^{*}$ intermediate, thus explaining the $\mathrm{CO}_{2} \mathrm{RR}$ enhancement and methane production. In the second example, the compositional and structural evolution of $\mathrm{Cu}_{100-\mathrm{x}} \mathrm{Zn}_{\mathrm{x}}$ NCs were monitored using XANES and EXAFS. ${ }^{369}$ The authors observed methane production for $\mathrm{Zn}$ contents lower than 50\%, while $\mathrm{CO}$ became the dominant product for higher $\mathrm{Zn}$ percentages. The composition and time dependent operando results (Figure 26C and $\mathrm{D}$ ) identified $\mathrm{Cu} / \mathrm{ZnO}$ as the active interface for methane production and show that there is an optimum of $30 \% \mathrm{Zn}$ in the material. At $\mathrm{Zn}$ concentrations higher than $70 \%$ the catalyst tends to form the $\mathrm{CuZn}$ alloy faster and drive the selectivity away from $\mathrm{CH}_{4}$ and mostly towards the CO. 

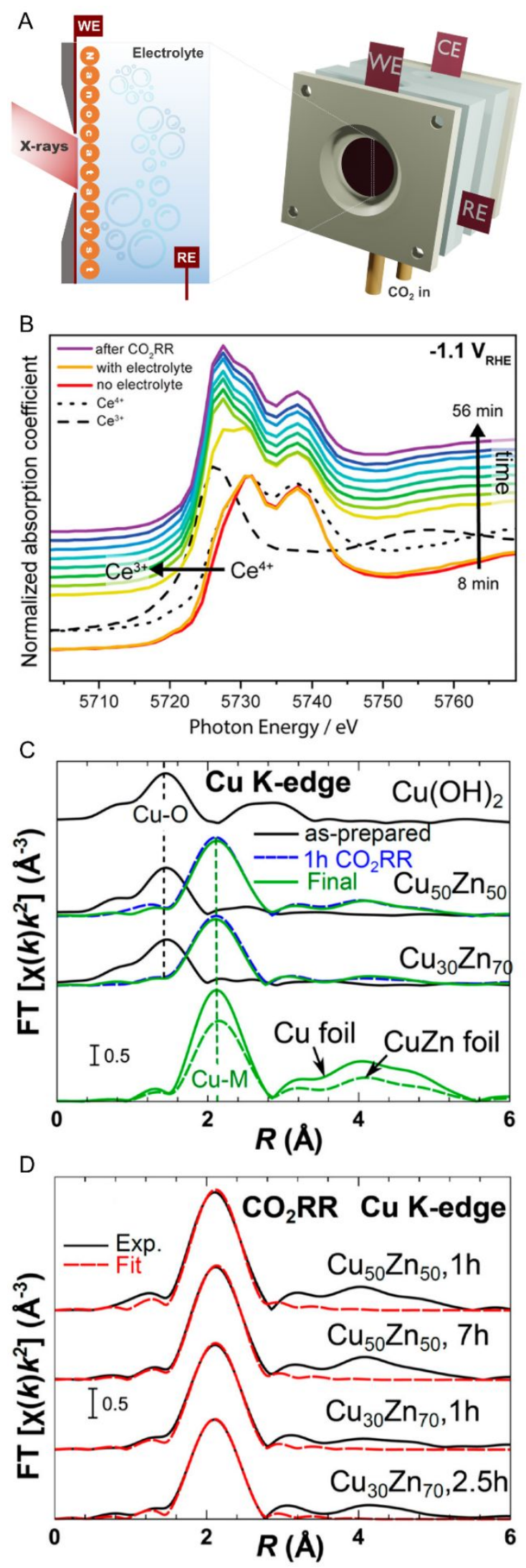

Figure 26. Operando XAS studies on NC electrocatalyst. (A) Schematic of the operando XAS cell (fluorescence geometry) utilized by Varandili et al. ${ }^{354}$ (B) $\mathrm{Ce}_{3}$-edge of $\mathrm{Cu} / \mathrm{CeO}_{2}$ evolving during $\mathrm{CO}_{2} \mathrm{RR}$. Adapted with permission from reference 354. Copyright 2019, 
American Chemical Society. (C) $\mathrm{Cu}$ EXAFS analysis during $\mathrm{CO}_{2} \mathrm{RR}$ of $\mathrm{Cu}_{100-\mathrm{x}} \mathrm{Zn}_{\mathrm{x}}$ nanocatalysts in the study by Jeon et al. ${ }^{369}$ and (D) EXAFS fitting for CuZn 50/50 and 30/70 displaying the evolution of the $\mathrm{Cu}-\mathrm{M}$ bond in longer $\mathrm{CO}_{2} \mathrm{RR}$ runs. Adapted with permission from reference 369. Copyright 2019, American Chemical Society.

While demonstrated to be a very powerful tool to monitor compositional changes of catalysts during operation, we would like highlight the importance of assuring that the X-Ray probe itself does not contribute to such changes. With the new generation of more brilliant synchrotron sources, which has tremendously improved signal and time resolution, a great deal of attention must be placed with this regard. ${ }^{381}$ While beam damage by X-Rays is more frequent in biological samples rather than in inorganic samples, systematic studies of how X-ray brilliance and photon flux affect the apparent behavior of the catalysts are always recommended. A non-focused beam might offer a solution in case of samples suffering from beam damage as recently suggest by Newton et al. ${ }^{381}$

\subsection{Electron microscopy}

Electron microscopy and predominantly TEM is an indispensable tool in characterizing colloidal NCs. High-resolution imaging and numerous diffraction techniques provide structural information about the as-synthesized NCs. Analytical capabilities of modern microscopes can provide elemental maps with EDX and EELS spectroscopy down to atomic spatial resolution. The specimen is typically exposed to the high vacuum of the microscope column during the analysis. However, thanks to recent improvements for the in situ instrumentation, the high vacuum around the specimen is no longer required and processes such as colloidal NC synthesis and their catalytic performance can be visualized in real-time under reaction conditions that closely resemble those of their bulk reaction cells. Two technologies to overcome the high vacuum limitation are commercialized: environmental TEM (E-TEM) and liquid cell TEM (LC-TEM). ${ }^{382}$

The specimen chamber in E-TEM systems is supplied with a gas of interest, differential pumping and apertures are then used to maintain the high vacuum in other parts of the column. The maximum pressure allowed in such systems is on the order of $10 \mathrm{mBar}$, which allows for measurements in gas atmosphere and low vapor pressure liquids. E-TEM allows for atomic resolution imaging and can provide valuable insights in gas-phase catalysis, especially combined with a heating stage. ${ }^{383-385}$ Its application for electrocatalysis is limited by the low- 
pressure requirements, yet a few examples exist. A model $\mathrm{Pt} / \mathrm{C}$ catalyst under conditions relevant to fuel cell operation has been studied with E-TEM. Yoshida et al. identified the migration of $\mathrm{Pt}$ on the $\mathrm{C}$ support followed by coalescence to be the major coarsening mechanism, where the carbon support, once hydrated in the atmosphere of water vapor, facilitates the Pt NC migration. ${ }^{386,387}$ It was further established by Luo et al., that the coalescence step is preceded by particle alignment to match the lattice orientation. ${ }^{388}$ Manganite catalysts for OER in perovskite and Ruddlesden-Popper phases have been studied by Mildner et al. with high-resolution E-TEM, where the electron beam in water vapor was used to induce positive local potentials on the electrode. ${ }^{389}$ By careful comparison of observed stability trends in E-TEM with details about the $\mathrm{O}$ and Mn oxidation state, provided by in situ EELS spectroscopy and results obtained in a reference electrochemical cell, they propose that the more ionic character of the $\mathrm{Mn}-\mathrm{O}$ bond is responsible for the increased stability in this type of catalyst. ${ }^{390}$ While studying stability in water vapor and driving electrochemistry with the electron beam of the microscope might seem rather disconnected from the conditions experienced by the catalyst under operation, this technique can still provide valuable insight into electrocatalyst stability.

It is worthwhile to notice that the solvent radiolysis driven by the electron irradiation is unavoidable; for example neat water produces a plethora of reactive species, ${ }^{391}$ among the most prominent are $\mathrm{H}_{3} \mathrm{O}^{+}, \mathrm{OH}, e_{\text {aq }}^{-}, \mathrm{H}^{\cdot}, \mathrm{OH}^{-}, \mathrm{H}_{2} \mathrm{O}_{2}$ and $\mathrm{H}_{2}$ (in descending order by the rate of generation). However, as the concentration of these species can be controlled (i.e. by the electron dose rate, accelerating voltage and the solution chemistry) and radical scavengers may be added to react preferentially with oxidizing or reducing species ${ }^{392,393}$, the beam-induced reductive environment can then be used to reduce metal ions, synthesize core-shell NCs or assist galvanic replacements ${ }^{394,395}$. Similarly, the beam-induced oxidative environment may be used to drive dissolution of colloidal NCs or to simulate the conditions of OER. ${ }^{389,390}$ With a deeper understanding of the underlying chemistry, the scope of this technique has the potential to become broader and broader. A neat example is a study by Liu et al. on the self-assembly of $\mathrm{Au}$ NCs where the hydrated electrons reduce the surface charge provided by the capping ligands and cause the self-assembly of the $\mathrm{NCs}^{396}$ On the other side, a different strategy is to minimize the electron beam effect by using low electron doses, highly sensitive cameras and performing relevant control experiments, to ensure that the observed process is not influenced by the electron irradiation. ${ }^{397}$ Working in the dose limiting regime limits the use of analytical 
techniques such as EDX and core-loss EELS, that typically require orders of magnitude larger doses than required for imaging.

The low operating pressures of open E-TEM are incompatible with the liquid phase of most common solvents and electrolytes. To overcome this limitation, closed cell systems are employed, where the specimen is separated by a thin membrane. The thinnest membranes, that can withstand the pressure gradient are made of graphene, providing the ultimate resolution in LC-TEM. ${ }^{398,399}$ Such level of detail has not been achieved yet in the context of monitoring NCs during electrocatalysis, where a more complex experimental setup is required.

Generally, patterned electrodes are incorporated in microfabricated $\mathrm{SiN}_{\mathrm{x}}$ liquid cells, ${ }^{400}$ providing a platform to study electrocatalysts under working conditions. With increased complexity of the liquid cell, one can simulate electrocatalyst operation directly at the cost of decreased resolution coming from the larger thickness of the liquid enclosure. Atomically resolved imaging in an electrochemical cell has not been demonstrated yet, as the achievable resolution in these thicker cells is limited by chromatic aberration and strong electron scattering. 382

With this technique, migration of Pt NCs on a $\mathrm{C}$ support and subsequent coarsening of the catalyst was imaged in real time in a reactor simulating a fuel cell. ${ }^{401} \mathrm{Zhu}$ et al. evaluated the morphology evolution of a Pt-Fe catalyst for ORR at various stages during potential cycling, which led to a better understanding of the potential-induced catalyst degradation. ${ }^{402}$ Moreover, Beermann et al. observed carbon support corrosion, particle migration and coalescence for octahedral Pt-Ni NCs under simulated ORR conditions. ${ }^{403}$ By correlating the applied potential to the catalyst morphology, a threshold potential for NC stability was established. Gradual and irreversible surface amorphization of a model $\mathrm{Co}_{3} \mathrm{O}_{4}$ catalyst during OER was observed by Ortiz Pena et al., confirming the oxy-hydroxide layer as the active form of catalyst (Figure 27A). ${ }^{404}$ Recently, several studies have focused on the transformation of $\mathrm{Cu} N C$ s under $\mathrm{CO}_{2} \mathrm{RR}$ conditions. Arán-Ais et al. observed the formation of cubic $\mathrm{Cu}_{2} \mathrm{O}$ particles from copper sulfate during electrochemical synthesis and their subsequent changes in a $\mathrm{CO}_{2}$-saturated aqueous electrolyte under reducing potential. ${ }^{405}$ In particular, they noticed dissolution of non-cubic particles while the cubic ones remained more stable (Figure 27B). In situ TEM has also been utilized by Li et al. to provide supporting evidence on the transformation of spherical $\mathrm{Cu}$ NCs into "scrambled" NCs during $\mathrm{CO}_{2} \mathrm{RR}$. These disordered structures were demonstrated to be the active catalyst favoring $\mathrm{C}-\mathrm{C}$ coupling. ${ }^{352}$ Finally, the underlying mechanism of this transformation was revealed by Vavra et al. by resolving individual particles throughout the process. ${ }^{351} \mathrm{The} \mathrm{Cu}$ NCs were shown to initially oxidize and partially dissolve in the electrolyte 
at open circuit voltage (OCV), followed by their re-deposition as larger $\mathrm{Cu}$ NCs. In a second stage, during $\mathrm{CO}_{2} \mathrm{RR}$, the remaining spherical NCs continued to dissolve and feed the growth of larger NCs in a process similar to Ostwald ripening (Figure 27C-E). These new insights were possible thanks to the development of a microfabricated electrochemical chip with glassy carbon working electrode specifically for in situ TEM under $\mathrm{CO}_{2} \mathrm{RR}$ conditions.
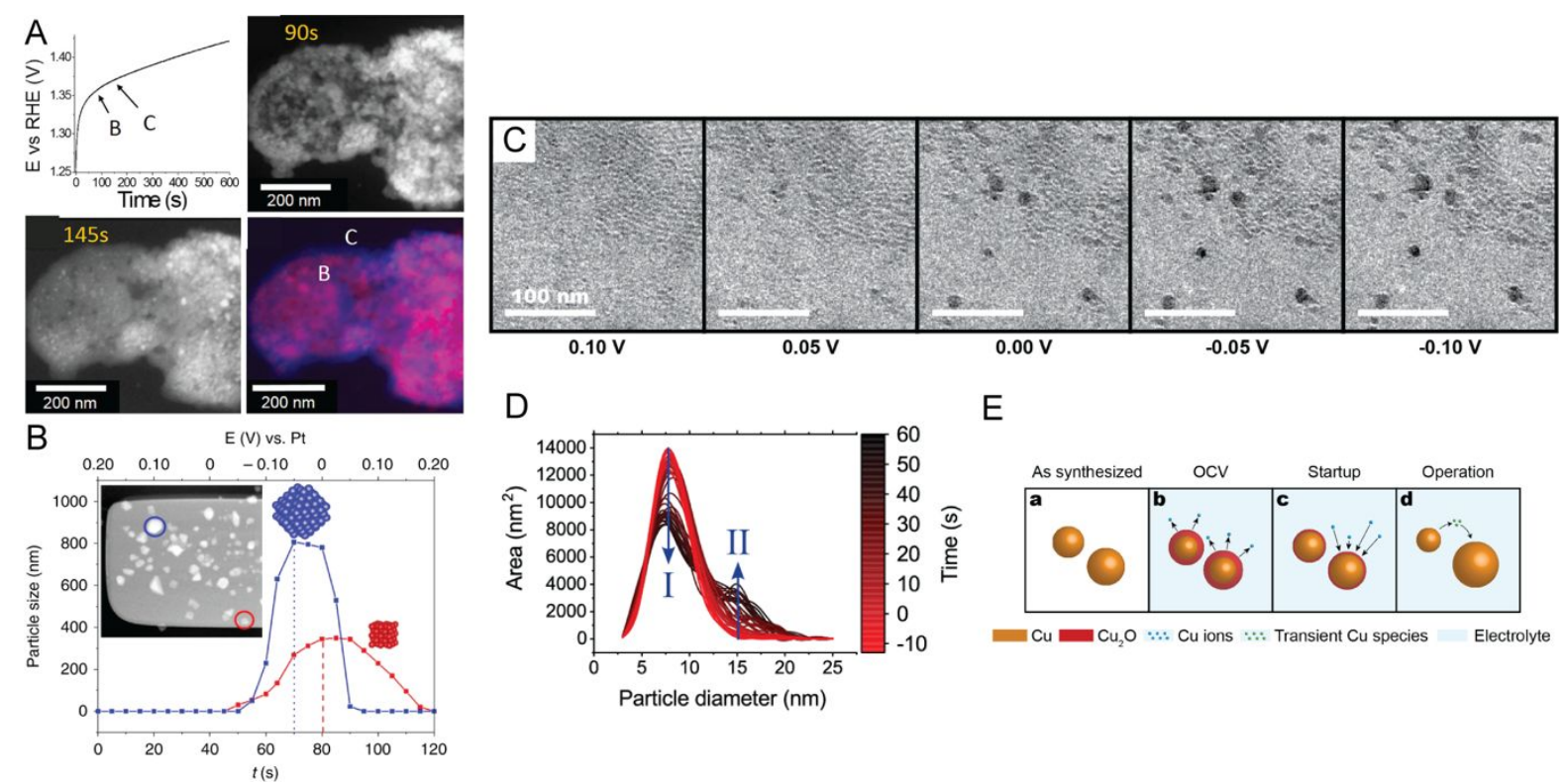

\section{Figure 27. Electrochemical in situ TEM to study catalyst transformation during} operation. (A) In situ electrochemical TEM chronopotentiometry OER experiment at $10 \mathrm{~mA}$ $\mathrm{cm}^{-2}$ in aqueous $0.1 \mathrm{M} \mathrm{KOH}$ electrolyte for $10 \mathrm{~min}$, where amorphization of the initial $\mathrm{Co}_{3} \mathrm{O}_{4}$ catalyst yields surface oxyhydroxide-like phase. STEM-HAADF images recorded at 90s and $145 \mathrm{~s}$ and superposition of both images evidencing the evolution of the area of the agglomerate (bottom right). Adapted with permission from reference 404. Copyright 2019, American Chemical Society. (B) Electrodeposition of $\mathrm{CO}_{2} \mathrm{RR}$ catalyst documenting increased stability of cube-shaped $\mathrm{Cu}_{2} \mathrm{O}$ particles. Adapted with permission from reference 405. Copyright 2020, Nature. (C) Snapshots from in situ TEM monitoring the transformation of $\mathrm{Cu}$ nanocatalysts into their active form during the startup phase of $\mathrm{CO}_{2} \mathrm{RR}$. (D) Particle size distribution of the same as a function of time at $-0.25 \mathrm{~V}_{\mathrm{RHE}}$ : some particles are growing (arrow II) while others shrink and eventually disappear (arrow I). (E) Schematic illustration of the solution-mediated Ostwald ripening process of $\mathrm{Cu}$ nanocatalysts during the startup phase of $\mathrm{CO}_{2} \mathrm{RR}$. Adapted with permission from reference 351. Copyright, 2020 Wiley. 


\section{Translation of fundamental studies on colloidal NC catalysts to industrially relevant conditions and devices}

One important aspect with respect to the testing of well-defined NCs is the tranfer of the knowledge collected under more ideal conditions, far from mass transport limitations (i.e. in RDE setups and H-cells), to devices which are more industrially relevant. Efforts in this direction have been recently increasing, especially with respect to ORR and $\mathrm{CO}_{2} \mathrm{RR}$.

In ORR, MEA-based setups are the ultimate testing station, as proton-exchange membrane fuel cells (PEMFC) rely on this configuration. Some of the promising Pt-based NCs (i.e. Pt-Co, Pt$\mathrm{Ni}$ and Ga-doped Pt-Ni) have been tested in a MEA cell (Table S2). ${ }^{406-410}$ While most of them were found to exhibit a superior activity compared to commercial $\mathrm{Pt} / \mathrm{C}$, the enhancement factor was much lower compared the one evaluated in a RDE setup. For example, the dealloyed Pt$\mathrm{Ni}$ nanoframes demonstrated only 3.5 and 6 times increase in mass and specific activity at $0.9 \mathrm{~V}_{\mathrm{RHE}}$ compared to the commercial catalyst, respectively. ${ }^{407,410}$ Instead, the increase in mass (36 times) and specific (22 times) activity in the RDE setup were 36 and 22 times, respectively. Similar observations were more recently made for Mo-doped $\mathrm{Pt}_{3} \mathrm{Ni}$ octahedra which showed 1.6 times mass activity enhancement vs. $\mathrm{Pt} / \mathrm{C}$ at $0.9 \mathrm{~V}_{\mathrm{RHE}}$ in the MEA setup compared to an outstanding 73 times enhancement in RDE setup. ${ }^{408}$

To be able to address this issue, one needs to understand what are the reasons behind this change of the catalyst performance in MEA compared to RDE setup. First of all, at high current density under $\mathrm{H}_{2} /$ Air flow and low Pt loading $\left(<0.1 \mathrm{mg}_{\mathrm{Pt}} / \mathrm{cm}^{2}\right)$, prominent voltage and power density losses emerge. ${ }^{410}$ Sulfonate groups from Nafion may also poison the Pt surface..$^{411}$ Kongkanand et al. have shown that losses mainly arise from local $\mathrm{O}_{2}$ mass transport resistance near the surface of $\mathrm{Pt}^{412,413}$ This becomes critical at electrochemically active surface areas (ECSA) lower than $40 \mathrm{~m}^{2} / \mathrm{g}_{\mathrm{Pt}}$, as inverse scaling between local mass transport resistance and surface roughness factor exists. This was further confirmed and expanded in a study by Schuler et al., where they used both multiscale modeling and MEA-based testing protocols to deconvolute various contributions to the local mass transport resistance origin. ${ }^{414}$ They conclude that the catalyst layer resistance is composed of two components: transport and interfacial, where the transport resistance through the ionomer thin-film or very local to the reaction site dominates. This is not the case for RDE studies, as mass transport conditions are intrinsically different between these two, serving as a key reason for difference in activity of well-structured catalysts in these devices. 
Finally, to further address the transfer from RDE to MEA cells, Martens et al. report on the protocol to establish a better correlation between testing in RDE and MEA setup. ${ }^{415} \mathrm{Using} \mathrm{Pt} / \mathrm{C}$ catalyst as a benchmark, they obtain a strict RDE protocol which is reproducible over different labs and demonstrates the same trends as with the use of MEA setup. Moreover, another cell geometry called floating electrode technique is proposed as an intermediate step between RDE and MEA, combining benefits of both, namely low catalyst loading and high current densities. With this in mind, we can conclude that, even though MEA implementation is challenging and it is probably not possible to get the record activities obtained in RDE, colloidal NCs are among the most active and durable materials towards ORR in PEMFC. More advances in the field are expected in upcoming years resulting from ongoing research devoted to their implementation in MEA setup. ${ }^{410}$

In $\mathrm{CO}_{2} \mathrm{RR}$, studies aimed at translating the results of fundamental studies in a H-cell to gasfed reactors capable of sustaining high current densities, which are relevant for industrial conditions, ${ }^{96}$ have recently started to populate the literature (Table S3).

In a recent work, De Gregorio et al. demonstrated that the facet-dependent selectivity of $\mathrm{Cu}$ nanocubes $\left(\mathrm{Cu}_{\mathrm{cub}}\right)$ and octahedra $\left(\mathrm{Cu}_{\text {oct }}\right)$ is retained when the NCs are loaded onto a GDE and measured in a gas flow cell (Figure 28). ${ }^{416} \mathrm{The} \mathrm{Cu}_{\mathrm{cub}}$ exhibit higher selectivity a much higher selectivity towards ethylene compared to the spheres $\left(\mathrm{Cu}_{\mathrm{sph}}\right)$ across all the potentials. This result points at the fact that the exposed (100) facets do play a role in directing selectivity, even under these more extreme conditions. Specifically, the conversion of $\mathrm{CO}_{2}$ to $\mathrm{C}_{2} \mathrm{H}_{4}$ ranged from $55 \%$ at $100 \mathrm{~mA} / \mathrm{cm}^{2}$ and $-0.65 \mathrm{~V}$ vs RHE to around $60 \%$ at $200 \mathrm{~mA} / \mathrm{cm}^{2}$ and $-0.70 \mathrm{~V}$ vs RHE, which is even superior to the maximum obtained in the H-cell for the same sample $(44 \%$ at $1.1 \mathrm{~V}_{\mathrm{RHE}}$, Table S3). As for the $\mathrm{Cu}_{\mathrm{oh}}$ 's, methane is the main hydrocarbon product, in line with the presence of the exposed (111) facets. This result strongly corroborates the importance of facet-control, as this product is normally suppressed at high $\mathrm{pH} .{ }^{417}$ The highest FE of $\sim 53 \%$ is obtained at $100 \mathrm{~mA} / \mathrm{cm}^{2}$ and $-0.91 \mathrm{~V}$ vs RHE, with the corresponding lowest $\mathrm{H}_{2}$ production (FE $\sim 22 \%$ ), which is again even higher than the maximum obtained in the H-cell for the sample with same size (34\% at $-1.25 \mathrm{~V}_{\mathrm{RHE}}$, Table S3).

While a truly quantitative comparison between H-cell and gas-fed flow cell is not possible because many differences between the two (i.e. electrolytes, mass and electron transport, $\mathrm{pH}$ gradients, applied potentials, etc..), these results confirm the importance of catalyst morphology even at such high current densities. They also highlight synergism between reactor and catalyst performance. Furthermore, the availability of the NCs as an ink also provides the opportunity to perform future studies focused on impact of the catalyst distribution within the 
GDE on the overall performance, something which is not accessible by other techniques such as thermal deposition. ${ }^{418}$
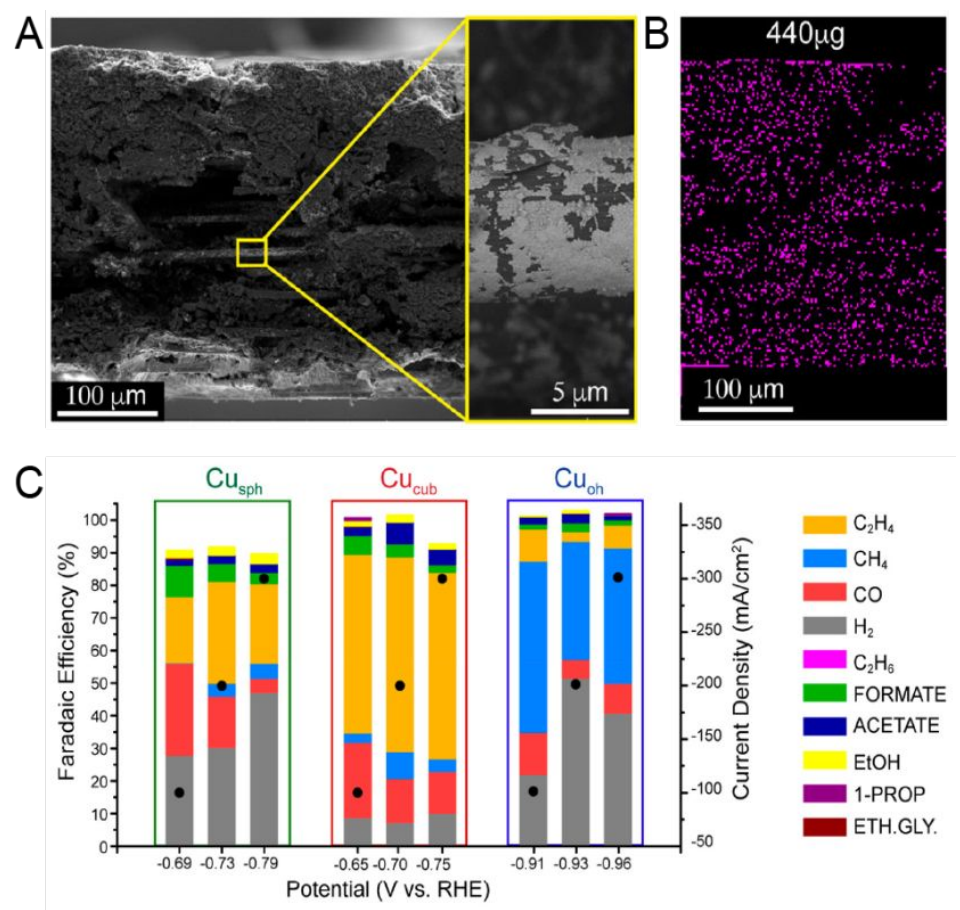

Figure 28. Cross-sectional (A) SEM images (with a magnification) and (B) EDX colored map of $440 \mu \mathrm{g} / \mathrm{cm}_{2} \mathrm{Cu}_{\text {cub }} \mathrm{NCs}$ deposited on the GDL by spray coating directly from their solution in hexane. (C) FEs vs potential for $\mathrm{Cu}_{\text {sph }}\left(200 \mu \mathrm{g} / \mathrm{cm}^{2}\right), \mathrm{Cu}_{\text {cub }}\left(250 \mu \mathrm{g} / \mathrm{cm}^{2}\right)$, and $\mathrm{Cu}_{\mathrm{oh}}$ $\left(50 \mu \mathrm{g} / \mathrm{cm}^{2}\right)$ deposited on a GDL and measured in the gas-fed flow cell in $1 \mathrm{M} \mathrm{KOH}$. Similar selectivities were obtained in $1.2 \mathrm{M} \mathrm{KHCO}_{3}$. Adapted with permission from reference 416. Copyright 2020, American Chemical Society.

The conclusions of Wang et al. also supported $\mathrm{C}_{2} \mathrm{H}_{4}$ formation with higher $\mathrm{FE}$ on $\mathrm{Cu}$ nanocubes $\left(\mathrm{C}_{2} \mathrm{H}_{4} \mathrm{FE}\right.$ of $\left.60 \%\right)$ than on $\mathrm{Cu}$ nanospheres $\left(\mathrm{C}_{2} \mathrm{H}_{4} \mathrm{FE}\right.$ of $\left.38 \%\right)$ in a gas-fed flow cell. ${ }^{419}$ In their recent work, Möller et al. compared the performance of cubic $\mathrm{Cu}_{2} \mathrm{O}$ NCs in the H-cell and gasfed flow cell. Concurring with earlier findings, they recorded higher $\mathrm{FE}$ and activity for $\mathrm{C}_{2+}$ products $\left(59 \%\right.$ at $300 \mathrm{~mA} / \mathrm{cm}^{2}$ current density) in the flow cell than the $\mathrm{H}$-cell $(48 \%$ at $15 \mathrm{~mA} / \mathrm{cm}^{2}$ current density). ${ }^{420}$

The next steps are now to work on improving the overall cell voltage and conversion efficiencies and to investigate catalyst stability and reconstruction processes in the GDE. Xray microscopy (XRM) might be interesting to be explored as an operando tool to study electrocatalysts in such configuration. ${ }^{421-423}$ XRM-based methods have mostly been utilized for batteries so far, with fewer examples in fuel cells. ${ }^{423-435}$ Some studies have applied XRM 
to characterize NCs. ${ }^{436-438}$ The big advantage of XRM is that different acquisition modes can be used to obtain compositional and structural information from tens of nanometers up to the centimeter scale. ${ }^{439-443}$ Therefore, such a tool would be highly complementary to in situ electron microscopy, which provides insight at the atomic scale. Another issue that needs to be addressed is the overall device stability and GDE flooding. ${ }^{444}$ The introduction of hydrophobic/hydrophilic block copolymers might be helpful towards this direction. ${ }^{35}$ Furthermore, switching towards MEA-based devices might be generally beneficial also in $\mathrm{CO}_{2} \mathrm{RR}$ as the absence of a liquid electrolyte could be beneficial both for catalyst and device stability.

\section{Conclusions and outlook}

The activity and selectivity of electrocatalysts strongly depends on their composition, size and shape. Therefore, controlling and tuning such parameters is of the utmost importance to establish mechanistic understanding, as well as to discover new catalysts. Additionally, the uniformity of these parameters across the entire batch of synthesized NCs is crucial when investigating catalyst reconstruction during operation.

In this review, we have highlighted the contribution of colloidal chemistry as a synthetic tool to access well-controlled and tunable electrocatalysts to drive $\mathrm{HER} / \mathrm{HOR}, \mathrm{OER} / \mathrm{ORR}, \mathrm{CO}_{2} \mathrm{RR}$, the formic acid and various alcohol oxidation reactions. The systematic variation of size and shape has revealed interesting correlations that would not have been otherwise discovered. For example, synergistic effects between size and shape have emerged such that the shape dictates the exposed facets and the size governs the facet ratio, thus providing an additional parameter to optimize reaction selectivity. Changing the composition or structural order while keeping the same size and morphology has facilitated the identification of the most active catalyst to drive a certain reaction. Tailoring interfaces between two or more metals and between metals and other supports has been demonstrated to be a powerful tool to discover unpredicted synergistic effects.

One major advantage of colloidal NCs is that they may be formulated as dispensable inks, and therefore can be easily integrated into different device configurations, from RDE and H-cells to GDE-based cells. Generally, together with the overall device architecture, it is clear that the utilized deposition method and electrode preparation might also impact the performance of the catalytic system. ${ }^{29,38,39,445-448}$ While the deposition should be performed in a way that the 
catalyst layer is as uniform as possible, intrinsic differences between various methods should also be taken into account, especially in the case of rough electrode surfaces. As a result, changing and comparing different deposition methods, which is feasible with NC inks, may serve as another way to tune the catalytic performance without changing the catalyst material or support itself.

To perform testing under both ideal and technologically relevant conditions has a double purpose. On one side, exposing identical catalysts to different reaction microenvironments can help to elucidate the effects of these environment on the reaction outcome. ${ }^{103,449}$ Additionally, testing under industrially relevant conditions can address the question of whether colloidal NCs serve only as model systems or if they also offer a technological solution. A few examples in the $\mathrm{CO}_{2} \mathrm{RR}$ field showing facet-dependent selectivity of colloidal NCs retained at high current density and the demonstrated synthesis scale-ups suggest that their use as commercial catalysts represents a concrete possibility. ${ }^{416,419,450-452}$ Considering these encouraging results, more studies to implement colloidal NC catalysts in industrial electrolyzers should be pursued.

As for the characterization, much can be learned by studying the catalyst as-synthesized and its changes after the reaction. Yet in situ techniques are the key to understand the catalyst synthesis and its function. Although in situ TEM is hindered by many practical limitations, the wealth of information provided by the technique makes it well worth the effort of finding a suitable approach to simulate conditions of the process in question. In situ TEM has assisted the mechanistic understanding of colloidal NC synthesis by providing real-time high-resolution images of individual particles in the early stages of their formation. The implementation of in situ electron microscopy as a technique for electrocatalyst characterization under operating conditions is very much at its infancy, yet the promise of understanding electrocatalyst stability and its active form is highly motivating. Among the X-ray based techniques, XAS and EXAFS are certainly the most common to study electrocatalysts under operation. In the future, XRM might be interesting to be explored as an operando tool to study electrocatalysts in GDE-based cells at high current densities. ${ }^{421-423}$ In the future, studies comparing results from in-situ TEM and operando X-Ray measurements will be helpful to further assess if any effect of the probing source is taking place. ${ }^{351}$

Regarding the surface ligands, investigations of their role and fate during electrocatalysis should certainly continue. New catalytic schemes where ligands play an active role are attracting increasing attention and merging the homogeneous and heterogeneous catalysis communities. While one can rely on the aforementioned in situ and operando measurements to study changes in the inorganic NC core, changes in the organic shell are more challenging 
to monitor due to the low concentration. In situ near ambient pressure X-ray photoemission spectroscopy (NAP-XPS) is a powerful tool for surface studies, yet deconvoluting the signal of the ligands in the presence of the electrolyte might be challenging. Electroanalytical methods, such as the quasi in situ impedance measurements utilized by Pankhurst et al. to investigate the fate of ligands during $\mathrm{CO}_{2} \mathrm{RR}$, and in situ vibrational spectroscopies (IR and Raman) might be more promising. ${ }^{88,300,453}$ The development of in situ solid-state NMR, in a similar fashion to what has been achieved in the battery field to study inorganic materials, might also offer great opportunities in the field of electrocatalysis with colloidal NCs. ${ }^{454-456}$ Finally, synthetic development will be crucial to exploit the full potential of colloidal NCs as electrocatalysts in the future. While a huge library of noble metal NCs with a large variety of structures and a continuously increasing compositional complexity is available in the literature, non-noble metal NCs are far less investigated and the level of shape control attainable for this class of NCs is still considerably scarce. ${ }^{17}$ For example, stabilization of high-index facets on NC surfaces (i.e. (751) for $\mathrm{Cu})^{204}$ or of metastable crystal structures, both of which are often desirable for activity and selectivity, is still limited to only a few systems, which are mostly based on noble metals. ${ }^{10,457}$ The synthesis of new NCs still proceeds via trial-and-error, therefore targeting compositions or shapes predicted by theory as well as tuning the distribution of each component within multimetallic NCs represent open challenges. Building a more fundamental understanding of the NC formation processes is therefore mandatory and in situ studies during the synthesis can accelerate progress in this direction. ${ }^{15,16,458,459}$ A "synthesisby-design" approach, where the reaction pathway and conditions to target the desired NC products can be easily identified, will render colloidal chemistry an even more powerful tool for catalyst investigation and discovery beyond the reactions discussed above. For example, the utilization of colloidal NC catalysts for emerging electrochemical reactions, including the nitrogen reduction reaction and organic transformations, where selectivity is an important challenge, can be anticipated in the near future. ${ }^{460,461}$

\section{ACKNOWLEDGEMENTS}

This work was financed by different funding sources. YTG and SBV are supported by the ERC Starting Grant ERC-HYCAT with agreement number 715634. JRP acknowledges the H2020 Marie Curie Individual Fellowship grant SURFCAT with agreement number 837378. DS and PY acknowledge the Sandoz foundation and Gaznat S.A. for financial support, respectively. 
VO and CK are financed by the Swiss National Science Foundation (SNSF) under AP Energy Grant, project number PYAPP2_166897/1. JV is supported by EPFL internal funding. 


\section{REFERENCES}

(1) Bell, A. T. The Impact of Nanoscience on Heterogeneous Catalysis. Science 2003, 299, $1688-1691$.

(2) Cargnello, M.; Fornasiero, P.; Gorte, R. J. Playing with Structures at the Nanoscale: Designing Catalysts by Manipulation of Clusters and Nanocrystals as Building Blocks. ChemPhysChem 2013, 14, 3869-3877.

(3) Cargnello, M. Colloidal Nanocrystals as Building Blocks for Well-Defined Heterogeneous Catalysts. Chem. Mater. 2019, 31, 576-596.

(4) Huang, J.; Buonsanti, R. Colloidal Nanocrystals as Heterogeneous Catalysts for Electrochemical CO2 Conversion. Chem. Mater. 2019, 31, 13-25.

(5) Ellis, P. R.; Brown, C. M.; Bishop, P. T.; Yin, J.; Cooke, K.; Terry, W. D.; Liu, J.; Yin, F.; Palmer, R. E. The Cluster Beam Route to Model Catalysts and Beyond. Faraday Discuss. 2016, 188, 39-56.

(6) Roldan Cuenya, B. Synthesis and Catalytic Properties of Metal Nanoparticles: Size, Shape, Support, Composition, and Oxidation State Effects. Thin Solid Films 2010, 518, 3127-3150.

(7) Gadiyar, C.; Loiudice, A.; Buonsanti, R. Colloidal Nanocrystals for Photoelectrochemical and Photocatalytic Water Splitting. J. Phys. D. Appl. Phys. 2017, $50,074006$.

(8) Rizo, R.; Roldan Cuenya, B. Shape-Controlled Nanoparticles as Anodic Catalysts in Low-Temperature Fuel Cells. ACS Energy Lett. 2019, 4, 1484-1495.

(9) Ruditskiy, A.; Peng, H.-C.; Xia, Y. Shape-Controlled Metal Nanocrystals for Heterogeneous Catalysis. Annu. Rev. Chem. Biomol. Eng. 2016, 7, 327-348.

(10) Xia, Y.; Xiong, Y.; Lim, B.; Skrabalak, S. E. Shape-Controlled Synthesis of Metal Nanocrystals: Simple Chemistry Meets Complex Physics? Angew. Chemie Int. Ed. 2009, 48, 60-103.

(11) Xia, Y.; Xia, X.; Peng, H.-C. Shape-Controlled Synthesis of Colloidal Metal Nanocrystals: Thermodynamic versus Kinetic Products. J. Am. Chem. Soc. 2015, 137, 7947-7966.

(12) Kwon, S. G.; Hyeon, T. Formation Mechanisms of Uniform Nanocrystals via HotInjection and Heat-Up Methods. Small 2011, 7, 2685-2702.

(13) Buonsanti, R.; Milliron, D. J. Chemistry of Doped Colloidal Nanocrystals. Chem. Mater. 
2013, 25, 1305-1317.

(14) Casavola, M.; Buonsanti, R.; Caputo, G.; Cozzoli, P. D. Colloidal Strategies for Preparing Oxide-Based Hybrid Nanocrystals. Eur. J. Inorg. Chem. 2008, 2008, 837854.

(15) Strach, M.; Mantella, V.; Pankhurst, J. R.; Iyengar, P.; Loiudice, A.; Das, S.; Corminboeuf, C.; van Beek, W.; Buonsanti, R. Insights into Reaction Intermediates to Predict Synthetic Pathways for Shape-Controlled Metal Nanocrystals. J. Am. Chem. Soc. 2019, 141, 16312-16322.

(16) Mantella, V.; Strach, M.; Frank, K.; Pankhurst, J. R.; Stoian, D.; Gadiyar, C.; Nickel, B.; Buonsanti, R. Polymer Lamellae as Reaction Intermediates in the Formation of Copper Nanospheres as Evidenced by In Situ X-ray Studies. Angew. Chemie Int. Ed. 2020, 59, 11627-11633.

(17) Mantella, V.; Castilla-Amorós, L.; Buonsanti, R. Shaping Non-Noble Metal Nanocrystals via Colloidal Chemistry. Chem. Sci. 2020, 11, 11394-11403.

(18) Callejas, J. F.; Read, C. G.; Roske, C. W.; Lewis, N. S.; Schaak, R. E. Synthesis, Characterization, and Properties of Metal Phosphide Catalysts for the HydrogenEvolution Reaction. Chem. Mater. 2016, 28, 6017-6044.

(19) Murray, C. B.; Norris, D. J.; Bawendi, M. G. Synthesis and Characterization of Nearly Monodisperse CdE (E = Sulfur, Selenium, Tellurium) Semiconductor Nanocrystallites. J. Am. Chem. Soc. 1993, 115, 8706-8715.

(20) Peng, X.; Manna, L.; Yang, W.; Wickham, J.; Scher, E.; Kadavanich, A.; Alivisatos, A. P. Shape Control of CdSe Nanocrystals. Nature 2000, 404, 59-61.

(21) Manna, L.; Milliron, D. J.; Meisel, A.; Scher, E. C.; Alivisatos, A. P. Controlled Growth of Tetrapod-Branched Inorganic Nanocrystals. Nat. Mater. 2003, 2, 382-385.

(22) Geisenhoff, J. Q.; Tamura, A. K.; Schimpf, A. M. Using Ligands to Control Reactivity, Size and Phase in the Colloidal Synthesis of WSe 2 Nanocrystals. Chem. Commun. 2019, $55,8856-8859$.

(23) Mahler, B.; Hoepfner, V.; Liao, K.; Ozin, G. A. Colloidal Synthesis of 1T-WS 2 and 2H-WS 2 Nanosheets: Applications for Photocatalytic Hydrogen Evolution. J. Am. Chem. Soc. 2014, 136, 14121-14127.

(24) Castilla-Amorós, L.; Stoian, D.; Pankhurst, J. R.; Varandili, S. B.; Buonsanti, R. Exploring the Chemical Reactivity of Gallium Liquid Metal Nanoparticles in Galvanic Replacement. J. Am. Chem. Soc. 2020, 142, 19283-19290.

(25) Varandili, S. B.; Huang, J.; Oveisi, E.; De Gregorio, G. L.; Mensi, M.; Strach, M.; Vavra, 
J.; Gadiyar, C.; Bhowmik, A.; Buonsanti, R. Synthesis of $\mathrm{Cu} / \mathrm{CeO} 2-\mathrm{x}$ Nanocrystalline Heterodimers with Interfacial Active Sites To Promote CO2 Electroreduction. ACS Catal. 2019, 9, 5035-5046.

(26) Varandili, S. B.; Stoian, D.; Vavra, J.; Pankhurst, J.; Buonsanti, R. Ligand-Mediated Formation of $\mathrm{Cu} / \mathrm{Metal}$ Oxide Hybrid Nanocrystals with Tunable Number of Interfaces. Chem. Sci. 2020.

(27) Huang, J.; Mensi, M.; Oveisi, E.; Mantella, V.; Buonsanti, R. Structural Sensitivities in Bimetallic Catalysts for Electrochemical $\mathrm{CO} 2$ Reduction Revealed by $\mathrm{Ag}-\mathrm{Cu}$ Nanodimers. J. Am. Chem. Soc. 2019, 141, 2490-2499.

(28) Cozzoli, P. D.; Pellegrino, T.; Manna, L. Synthesis, Properties and Perspectives of Hybrid Nanocrystal Structures. Chem. Soc. Rev. 2006, 35, 1195.

(29) Yang, J.; Choi, M. K.; Kim, D.-H.; Hyeon, T. Designed Assembly and Integration of Colloidal Nanocrystals for Device Applications. Adv. Mater. 2016, 28, 1176-1207.

(30) Boles, M. A.; Engel, M.; Talapin, D. V. Self-Assembly of Colloidal Nanocrystals: From Intricate Structures to Functional Materials. Chem. Rev. 2016, 116, 11220-11289.

(31) Dong, A.; Chen, J.; Vora, P. M.; Kikkawa, J. M.; Murray, C. B. Binary Nanocrystal Superlattice Membranes Self-Assembled at the Liquid-Air Interface. Nature 2010, 466, 474-477.

(32) Yuan, J.; Bi, C.; Wang, S.; Guo, R.; Shen, T.; Zhang, L.; Tian, J. Spray Coating Technologies: Spray-Coated Colloidal Perovskite Quantum Dot Films for Highly Efficient Solar Cells (Adv. Funct. Mater. 49/2019). Adv. Funct. Mater. 2019, 29, 1970337.

(33) Soliwoda, K.; Rosowski, M.; Tomaszewska, E.; Tkacz-Szczesna, B.; Celichowski, G.; Grobelny, J. Electrospray Deposition of Gold Nanoparticles from Aqueous Colloids on Solid Substrates. Colloids Surfaces A Physicochem. Eng. Asp. 2015, 486, 211-217.

(34) Brown, N. A.; Zhu, Y.; German, G. K.; Yong, X.; Chiarot, P. R. Electrospray Deposit Structure of Nanoparticle Suspensions. J. Electrostat. 2017, 90, 67-73.

(35) García de Arquer, F. P.; Dinh, C.-T.; Ozden, A.; Wicks, J.; McCallum, C.; Kirmani, A. R.; Nam, D.-H.; Gabardo, C.; Seifitokaldani, A.; Wang, X.; Li, Y. C.; Li, F.; Edwards, J.; Richter, L. J.; Thorpe, S. J.; Sinton, D.; Sargent, E. H. CO2 Electrolysis to Multicarbon Products at Activities Greater than 1 A Cm-2. Science 2020, 367, 661666.

(36) Corradini, P. G.; Pires, F. I.; Paganin, V. A.; Perez, J.; Antolini, E. Effect of the Relationship between Particle Size, Inter-Particle Distance, and Metal Loading of 
Carbon Supported Fuel Cell Catalysts on Their Catalytic Activity. J. Nanoparticle Res. 2012, 14, 1080 .

(37) Eklöf, J.; Gschneidtner, T.; Lara-Avila, S.; Nygård, K.; Moth-Poulsen, K. Controlling Deposition of Nanoparticles by Tuning Surface Charge of $\mathrm{SiO} 2$ by Surface Modifications. RSC Adv. 2016, 6, 104246-104253.

(38) Speder, J.; Spanos, I.; Zana, A.; Kirkensgaard, J. J. K.; Mortensen, K.; Altmann, L.; Bäumer, M.; Arenz, M. From Single Crystal Model Catalysts to Systematic Studies of Supported Nanoparticles. Surf. Sci. 2015, 631, 278-284.

(39) Mistry, H.; Behafarid, F.; Reske, R.; Varela, A. S.; Strasser, P.; Roldan Cuenya, B. Tuning Catalytic Selectivity at the Mesoscale via Interparticle Interactions. ACS Catal. 2016, 6, 1075-1080.

(40) Kim, D.; Resasco, J.; Yu, Y.; Asiri, A. M.; Yang, P. Synergistic Geometric and Electronic Effects for Electrochemical Reduction of Carbon Dioxide Using GoldCopper Bimetallic Nanoparticles. Nat. Commun. 2014, 5, 4948.

(41) Rossi, L. M.; Fiorio, J. L.; Garcia, M. A. S.; Ferraz, C. P. The Role and Fate of Capping Ligands in Colloidally Prepared Metal Nanoparticle Catalysts. Dalt. Trans. 2018, 47, 5889-5915.

(42) Reichenberger, S.; Marzun, G.; Muhler, M.; Barcikowski, S. Perspective of Surfactant-Free Colloidal Nanoparticles in Heterogeneous Catalysis. ChemCatChem 2019, 11, 4489-4518.

(43) Niu, Z.; Li, Y. Removal and Utilization of Capping Agents in Nanocatalysis. Chem. Mater. 2014, 26, 72-83.

(44) Ung, D.; Murphy, I. A.; Cossairt, B. M. Designing Nanoparticle Interfaces for InnerSphere Catalysis. Dalt. Trans. 2020, 49, 4995-5005.

(45) Niu, Z.; Becknell, N.; Yu, Y.; Kim, D.; Chen, C.; Kornienko, N.; Somorjai, G. A.; Yang, P. Anisotropic Phase Segregation and Migration of Pt in Nanocrystals En Route to Nanoframe Catalysts. Nat. Mater. 2016, 15, 1188-1194.

(46) Li, D.; Wang, C.; Tripkovic, D.; Sun, S.; Markovic, N. M.; Stamenkovic, V. R. Surfactant Removal for Colloidal Nanoparticles from Solution Synthesis: The Effect on Catalytic Performance. ACS Catal. 2012, 2, 1358-1362.

(47) Cargnello, M.; Chen, C.; Diroll, B. T.; Doan-Nguyen, V. V. T.; Gorte, R. J.; Murray, C. B. Efficient Removal of Organic Ligands from Supported Nanocrystals by Fast Thermal Annealing Enables Catalytic Studies on Well-Defined Active Phases. J. Am. Chem. Soc. 2015, 137, 6906-6911. 
(48) Popczun, E. J.; Read, C. G.; Roske, C. W.; Lewis, N. S.; Schaak, R. E. Highly Active Electrocatalysis of the Hydrogen Evolution Reaction by Cobalt Phosphide Nanoparticles. Angew. Chemie Int. Ed. 2014, 53, 5427-5430.

(49) Wu, L.; Li, Q.; Wu, C. H.; Zhu, H.; Mendoza-Garcia, A.; Shen, B.; Guo, J.; Sun, S. Stable Cobalt Nanoparticles and Their Monolayer Array as an Efficient Electrocatalyst for Oxygen Evolution Reaction. J. Am. Chem. Soc. 2015, 137, 7071-7074.

(50) Zhu, H.; Zhang, S.; Huang, Y.-X.; Wu, L.; Sun, S. Monodisperse MxFe3-XO4 (M = Fe, $\mathrm{Cu}, \mathrm{Co}, \mathrm{Mn})$ Nanoparticles and Their Electrocatalysis for Oxygen Reduction Reaction. Nano Lett. 2013, 13, 2947-2951.

(51) Guo, S.; Zhang, S.; Wu, L.; Sun, S. Co/CoO Nanoparticles Assembled on Graphene for Electrochemical Reduction of Oxygen. Angew. Chemie Int. Ed. 2012, 51, 11770-11773.

(52) Liu, Y.; Li, D.; Stamenkovic, V. R.; Soled, S.; Henao, J. D.; Sun, S. Synthesis of Pt3Sn Alloy Nanoparticles and Their Catalysis for Electro-Oxidation of CO and Methanol. ACS Catal. 2011, 1, 1719-1723.

(53) Cao, Z.; Kim, D.; Hong, D.; Yu, Y.; Xu, J.; Lin, S.; Wen, X.; Nichols, E. M.; Jeong, K.; Reimer, J. A.; Yang, P.; Chang, C. J. A Molecular Surface Functionalization Approach to Tuning Nanoparticle Electrocatalysts for Carbon Dioxide Reduction. J. Am. Chem. Soc. 2016, 138, 8120-8125.

(54) Lu, L.; Zou, S.; Zhou, Y.; Liu, J.; Li, R.; Xu, Z.; Xiao, L.; Fan, J. Ligand-Regulated ORR Activity of $\mathrm{Au}$ Nanoparticles in Alkaline Medium: The Importance of Surface Coverage of Ligands. Catal. Sci. Technol. 2018, 8, 746-754.

(55) Sellin, R.; Grolleau, C.; Arrii-Clacens, S.; Pronier, S.; Clacens, J.-M.; Coutanceau, C.; Léger, J.-M. Effects of Temperature and Atmosphere on Carbon-Supported Platinum Fuel Cell Catalysts. J. Phys. Chem. C 2009, 113, 21735-21744.

(56) Mohapatra, P.; Shaw, S.; Mendivelso-Perez, D.; Bobbitt, J. M.; Silva, T. F.; Naab, F.; Yuan, B.; Tian, X.; Smith, E. A.; Cademartiri, L. Calcination Does Not Remove All Carbon from Colloidal Nanocrystal Assemblies. Nat. Commun. 2017, 8, 2038.

(57) Cademartiri, L.; Ghadimi, A.; Ozin, G. A. Nanocrystal Plasma Polymerization: From Colloidal Nanocrystals to Inorganic Architectures. Acc. Chem. Res. 2008, 41, 18201830.

(58) Shaw, S.; Colaux, J. L.; Hay, J. L.; Peiris, F. C.; Cademartiri, L. Building Materials from Colloidal Nanocrystal Arrays: Evolution of Structure, Composition, and Mechanical Properties upon the Removal of Ligands by O2 Plasma. Adv. Mater. 2016, 28, 89008905. 
(59) Gehl, B.; Frömsdorf, A.; Aleksandrovic, V.; Schmidt, T.; Pretorius, A.; Flege, J.-I.; Bernstorff, S.; Rosenauer, A.; Falta, J.; Weller, H.; Bäumer, M. Structural and Chemical Effects of Plasma Treatment on Close-Packed Colloidal Nanoparticle Layers. $A d v$. Funct. Mater. 2008, 18, 2398-2410.

(60) Gao, D.; Zegkinoglou, I.; Divins, N. J.; Scholten, F.; Sinev, I.; Grosse, P.; Roldan Cuenya, B. Plasma-Activated Copper Nanocube Catalysts for Efficient Carbon Dioxide Electroreduction to Hydrocarbons and Alcohols. ACS Nano 2017, 11, 4825-4831.

(61) Shaw, S.; Tian, X.; Silva, T. F.; Bobbitt, J. M.; Naab, F.; Rodrigues, C. L.; Smith, E. A.; Cademartiri, L. Selective Removal of Ligands from Colloidal Nanocrystal Assemblies with Non-Oxidizing He Plasmas. Chem. Mater. 2018, 30, 5961-5967.

(62) Mistry, H.; Varela, A. S.; Bonifacio, C. S.; Zegkinoglou, I.; Sinev, I.; Choi, Y.-W.; Kisslinger, K.; Stach, E. A.; Yang, J. C.; Strasser, P.; Roldan Cuenya, B. Highly Selective Plasma-Activated Copper Catalysts for Carbon Dioxide Reduction to Ethylene. Nat. Commun. 2016, 7, 12123.

(63) Huang, J.; Hörmann, N.; Oveisi, E.; Loiudice, A.; De Gregorio, G. L.; Andreussi, O.; Marzari, N.; Buonsanti, R. Potential-Induced Nanoclustering of Metallic Catalysts during Electrochemical CO2 Reduction. Nat. Commun. 2018, 9, 3117.

(64) Iyengar, P.; Huang, J.; De Gregorio, G. L.; Gadiyar, C.; Buonsanti, R. Size Dependent Selectivity of $\mathrm{Cu}$ Nano-Octahedra Catalysts for the Electrochemical Reduction of $\mathrm{CO} 2$ to CH4. Chem. Commun. 2019, 55, 8796-8799.

(65) Shaw, S.; Yuan, B.; Tian, X.; Miller, K. J.; Cote, B. M.; Colaux, J. L.; Migliori, A.; Panthani, M. G.; Cademartiri, L. Building Materials from Colloidal Nanocrystal Arrays: Preventing Crack Formation during Ligand Removal by Controlling Structure and Solvation. Adv. Mater. 2016, 28, 8892-8899.

(66) Elliott, E. W.; Glover, R. D.; Hutchison, J. E. Removal of Thiol Ligands from SurfaceConfined Nanoparticles without Particle Growth or Desorption. ACS Nano 2015, 9, $3050-3059$.

(67) Zhong, R.-Y.; Sun, K.-Q.; Hong, Y.-C.; Xu, B.-Q. Impacts of Organic Stabilizers on Catalysis of Au Nanoparticles from Colloidal Preparation. ACS Catal. 2014, 4, 39823993.

(68) Aliaga, C.; Park, J. Y.; Yamada, Y.; Lee, H. S.; Tsung, C.-K.; Yang, P.; Somorjai, G. A. Sum Frequency Generation and Catalytic Reaction Studies of the Removal of Organic Capping Agents from Pt Nanoparticles by UV-Ozone Treatment. J. Phys. Chem. C 2009, 113, 6150-6155. 
(69) Lopez-Sanchez, J. A.; Dimitratos, N.; Hammond, C.; Brett, G. L.; Kesavan, L.; White, S.; Miedziak, P.; Tiruvalam, R.; Jenkins, R. L.; Carley, A. F.; Knight, D.; Kiely, C. J.; Hutchings, G. J. Facile Removal of Stabilizer-Ligands from Supported Gold Nanoparticles. Nat. Chem. 2011, 3, 551-556.

(70) Huang, Y.; Liu, W.; Cheng, H.; Yao, T.; Yang, L.; Bao, J.; Huang, T.; Sun, Z.; Jiang, Y.; Wei, S. Solvent-Induced Desorption of Alkanethiol Ligands from Au Nanoparticles. Phys. Chem. Chem. Phys. 2016, 18, 15927-15933.

(71) Krier, J. M.; Michalak, W. D.; Baker, L. R.; An, K.; Komvopoulos, K.; Somorjai, G. A. Sum Frequency Generation Vibrational Spectroscopy of Colloidal Platinum Nanoparticle Catalysts: Disordering versus Removal of Organic Capping. J. Phys. Chem. C 2012, 116, 17540-17546.

(72) Richter, T. V.; Stelzl, F.; Schulz-Gericke, J.; Kerscher, B.; Würfel, U.; Niggemann, M.; Ludwigs, S. Room Temperature Vacuum-Induced Ligand Removal and Patterning of ZnOnanoparticles: From Semiconducting Films towards Printed Electronics. J. Mater. Chem. 2010, 20, 874-879.

(73) Nelson, A.; Zong, Y.; Fritz, K. E.; Suntivich, J.; Robinson, R. D. Assessment of Soft Ligand Removal Strategies: Alkylation as a Promising Alternative to High-Temperature Treatments for Colloidal Nanoparticle Surfaces. ACS Mater. Lett. 2019, 1, 177-184.

(74) Ansar, S. M.; Ameer, F. S.; Hu, W.; Zou, S.; Pittman, C. U.; Zhang, D. Removal of Molecular Adsorbates on Gold Nanoparticles Using Sodium Borohydride in Water. Nano Lett. 2013, 13, 1226-1229.

(75) Donoeva, B.; de Jongh, P. E. Colloidal Au Catalyst Preparation: Selective Removal of Polyvinylpyrrolidone from Active Au Sites. ChemCatChem 2018, 10, 989-997.

(76) Schrader, I.; Warneke, J.; Neumann, S.; Grotheer, S.; Swane, A. A.; Kirkensgaard, J. J. K.; Arenz, M.; Kunz, S. Surface Chemistry of "Unprotected" Nanoparticles: A Spectroscopic Investigation on Colloidal Particles. J. Phys. Chem. C 2015, 119, 1765517661.

(77) Zhang, Z.; Chi, M.; Veith, G. M.; Zhang, P.; Lutterman, D. A.; Rosenthal, J.; Overbury, S. H.; Dai, S.; Zhu, H. Rational Design of Bi Nanoparticles for Efficient Electrochemical CO 2 Reduction: The Elucidation of Size and Surface Condition Effects. ACS Catal. 2016, 6, 6255-6264.

(78) Shi, Y.; Lyu, Z.; Cao, Z.; Xie, M.; Xia, Y. How to Remove the Capping Agent from Pd Nanocubes without Destructing Their Surface Structure for the Maximization of Catalytic Activity? Angew. Chemie Int. Ed. 2020, anie.202006011. 
(79) Fan, Q.; Liu, K.; Liu, Z.; Liu, H.; Zhang, L.; Zhong, P.; Gao, C. A Ligand-Exchange Route to Nobel Metal Nanocrystals with a Clean Surface for Enhanced Optical and Catalytic Properties. Part. Part. Syst. Charact. 2017, 34, 1700075.

(80) Zhang, L.; Roling, L. T.; Wang, X.; Vara, M.; Chi, M.; Liu, J.; Choi, S.-I.; Park, J.; Herron, J. A.; Xie, Z.; Mavrikakis, M.; Xia, Y. Platinum-Based Nanocages with Subnanometer-Thick Walls and Well-Defined, Controllable Facets. Science 2015, 349, $412-416$.

(81) Doris, S. E.; Lynch, J. J.; Li, C.; Wills, A. W.; Urban, J. J.; Helms, B. A. Mechanistic Insight into the Formation of Cationic Naked Nanocrystals Generated under Equilibrium Control. J. Am. Chem. Soc. 2014, 136, 15702-15710.

(82) Henckel, D. A.; Lenz, O.; Cossairt, B. M. Effect of Ligand Coverage on Hydrogen Evolution Catalyzed by Colloidal WSe2. ACS Catal. 2017, 7, 2815-2820.

(83) Rosen, E. L.; Buonsanti, R.; Llordes, A.; Sawvel, A. M.; Milliron, D. J.; Helms, B. A. Exceptionally Mild Reactive Stripping of Native Ligands from Nanocrystal Surfaces by Using Meerwein's Salt. Angew. Chemie Int. Ed. 2012, 51, 684-689.

(84) Ung, D.; Cossairt, B. M. Effect of Surface Ligands on CoP for the Hydrogen Evolution Reaction. ACS Appl. Energy Mater. 2019, 2, 1642-1645.

(85) Dong, A.; Ye, X.; Chen, J.; Kang, Y.; Gordon, T.; Kikkawa, J. M.; Murray, C. B. A Generalized Ligand-Exchange Strategy Enabling Sequential Surface Functionalization of Colloidal Nanocrystals. J. Am. Chem. Soc. 2011, 133, 998-1006.

(86) Lu, L.; Lou, B.; Zou, S.; Kobayashi, H.; Liu, J.; Xiao, L.; Fan, J. Robust Removal of Ligands from Noble Metal Nanoparticles by Electrochemical Strategies. ACS Catal. 2018, 8, 8484-8492.

(87) Manthiram, K.; Surendranath, Y.; Alivisatos, A. P. Dendritic Assembly of Gold Nanoparticles during Fuel-Forming Electrocatalysis. J. Am. Chem. Soc. 2014, 136, $7237-7240$.

(88) Pankhurst, J. R.; Iyengar, P.; Loiudice, A.; Mensi, M.; Buonsanti, R. Metal-Ligand Bond Strength Determines the Fate of Organic Ligands on the Catalyst Surface during the Electrochemical CO2 Reduction Reaction. Chem. Sci. 2020, 11, 9296-9302.

(89) Arán-Ais, R. M.; Yu, Y.; Hovden, R.; Solla-Gullón, J.; Herrero, E.; Feliu, J. M.; Abruña, H. D. Identical Location Transmission Electron Microscopy Imaging of Site-Selective Pt Nanocatalysts: Electrochemical Activation and Surface Disordering. J. Am. Chem. Soc. 2015, 137, 14992-14998.

(90) Liang, S.; Altaf, N.; Huang, L.; Gao, Y.; Wang, Q. Electrolytic Cell Design for 
Electrochemical CO2 Reduction. J. CO2 Util. 2020, 35, 90-105.

(91) Clark, E. L.; Bell, A. T. Direct Observation of the Local Reaction Environment during the Electrochemical Reduction of CO2. J. Am. Chem. Soc. 2018, 140, 7012-7020.

(92) Bondue, C. J.; Calle-Vallejo, F.; Figueiredo, M. C.; Koper, M. T. M. Structural Principles to Steer the Selectivity of the Electrocatalytic Reduction of Aliphatic Ketones on Platinum. Nat. Catal. 2019, 2, 243-250.

(93) Wang, X.; de Araújo, J. F.; Ju, W.; Bagger, A.; Schmies, H.; Kühl, S.; Rossmeisl, J.; Strasser, P. Mechanistic Reaction Pathways of Enhanced Ethylene Yields during Electroreduction of $\mathrm{CO} 2-\mathrm{CO} \mathrm{Co}-\mathrm{Feeds}$ on $\mathrm{Cu}$ and $\mathrm{Cu}$-Tandem Electrocatalysts. Nat. Nanotechnol. 2019, 14, 1063-1070.

(94) Bard, A. J.; Faulkner, L. R. Electrochemical Methods: Fundamentals and Applications, 2nd ed; Wiley, 2000.

(95) Clark, E. L.; Resasco, J.; Landers, A.; Lin, J.; Chung, L.-T.; Walton, A.; Hahn, C.; Jaramillo, T. F.; Bell, A. T. Standards and Protocols for Data Acquisition and Reporting for Studies of the Electrochemical Reduction of Carbon Dioxide. ACS Catal. 2018, 8, 6560-6570.

(96) Burdyny, T.; Smith, W. A. CO2 Reduction on Gas-Diffusion Electrodes and Why Catalytic Performance Must Be Assessed at Commercially-Relevant Conditions. Energy Environ. Sci. 2019, 12, 1442-1453.

(97) Higgins, D.; Hahn, C.; Xiang, C.; Jaramillo, T. F.; Weber, A. Z. Gas-Diffusion Electrodes for Carbon Dioxide Reduction: A New Paradigm. ACS Energy Lett. 2019, 4, 317-324.

(98) Möller, T.; Ju, W.; Bagger, A.; Wang, X.; Luo, F.; Ngo Thanh, T.; Varela, A. S.; Rossmeis1, J.; Strasser, P. Efficient CO2 to CO Electrolysis on Solid Ni-N-C Catalysts at Industrial Current Densities. Energy Environ. Sci. 2019, 12, 640-647.

(99) Endrődi, B.; Kecsenovity, E.; Samu, A.; Darvas, F.; Jones, R. V.; Török, V.; Danyi, A.; Janáky, C. Multilayer Electrolyzer Stack Converts Carbon Dioxide to Gas Products at High Pressure with High Efficiency. ACS Energy Lett. 2019, 4, 1770-1777.

(100) Ma, M.; Clark, E. L.; Therkildsen, K. T.; Dalsgaard, S.; Chorkendorff, I.; Seger, B. Insights into the Carbon Balance for $\mathrm{CO} 2$ Electroreduction on $\mathrm{Cu}$ Using Gas Diffusion Electrode Reactor Designs. Energy Environ. Sci. 2020, 13, 977-985.

(101) Weng, L.-C.; Bell, A. T.; Weber, A. Z. Towards Membrane-Electrode Assembly Systems for CO2 Reduction: A Modeling Study. Energy Environ. Sci. 2019, 12, 19501968. 
(102) Ott, S.; Orfanidi, A.; Schmies, H.; Anke, B.; Nong, H. N.; Hübner, J.; Gernert, U.; Gliech, M.; Lerch, M.; Strasser, P. Ionomer Distribution Control in Porous CarbonSupported Catalyst Layers for High-Power and Low Pt-Loaded Proton Exchange Membrane Fuel Cells. Nat. Mater. 2020, 19, 77-85.

(103) Gabardo, C. M.; O’Brien, C. P.; Edwards, J. P.; McCallum, C.; Xu, Y.; Dinh, C.-T.; Li, J.; Sargent, E. H.; Sinton, D. Continuous Carbon Dioxide Electroreduction to Concentrated Multi-Carbon Products Using a Membrane Electrode Assembly. Joule 2019, 3, 2777-2791.

(104) Zhu, J.; Hu, L.; Zhao, P.; Lee, L. Y. S.; Wong, K.-Y. Recent Advances in Electrocatalytic Hydrogen Evolution Using Nanoparticles. Chem. Rev. 2020, 120, 851918.

(105) Davydova, E. S.; Mukerjee, S.; Jaouen, F.; Dekel, D. R. Electrocatalysts for Hydrogen Oxidation Reaction in Alkaline Electrolytes. ACS Catal. 2018, 8, 6665-6690.

(106) Seh, Z. W.; Kibsgaard, J.; Dickens, C. F.; Chorkendorff, I.; Nørskov, J. K.; Jaramillo, T. F. Combining Theory and Experiment in Electrocatalysis: Insights into Materials Design. Science 2017, 355, eaad4998.

(107) Durst, J.; Siebel, A.; Simon, C.; Hasché, F.; Herranz, J.; Gasteiger, H. A. New Insights into the Electrochemical Hydrogen Oxidation and Evolution Reaction Mechanism. Energy Environ. Sci. 2014, 7, 2255-2260.

(108) Ledendecker, M.; Mondschein, J. S.; Kasian, O.; Geiger, S.; Göhl, D.; Schalenbach, M.; Zeradjanin, A.; Cherevko, S.; Schaak, R. E.; Mayrhofer, K. Stability and Activity of Non-Noble-Metal-Based Catalysts Toward the Hydrogen Evolution Reaction. Angew. Chemie Int. Ed. 2017, 56, 9767-9771.

(109) Ohyama, J.; Sato, T.; Yamamoto, Y.; Arai, S.; Satsuma, A. Size Specifically High Activity of Ru Nanoparticles for Hydrogen Oxidation Reaction in Alkaline Electrolyte. J. Am. Chem. Soc. 2013, 135, 8016-8021.

(110) Lu, Q.; Wang, A.-L.; Gong, Y.; Hao, W.; Cheng, H.; Chen, J.; Li, B.; Yang, N.; Niu, W.; Wang, J.; Yu, Y.; Zhang, X.; Chen, Y.; Fan, Z.; Wu, X.-J.; Chen, J.; Luo, J.; Li, S.; Gu, L.; Zhang, H. Crystal Phase-Based Epitaxial Growth of Hybrid Noble Metal Nanostructures on 4H/Fcc Au Nanowires. Nat. Chem. 2018, 10, 456-461.

(111) Cao, Z.; Chen, Q.; Zhang, J.; Li, H.; Jiang, Y.; Shen, S.; Fu, G.; Lu, B.; Xie, Z.; Zheng, L. Platinum-Nickel Alloy Excavated Nano-Multipods with Hexagonal Close-Packed Structure and Superior Activity towards Hydrogen Evolution Reaction. Nat. Commun. 2017, $8,15131$. 
(112) Alinezhad, A.; Gloag, L.; Benedetti, T. M.; Cheong, S.; Webster, R. F.; Roelsgaard, M.; Iversen, B. B.; Schuhmann, W.; Gooding, J. J.; Tilley, R. D. Direct Growth of Highly Strained Pt Islands on Branched Ni Nanoparticles for Improved Hydrogen Evolution Reaction Activity. J. Am. Chem. Soc. 2019, 141, 16202-16207.

(113) Li, Z.; Yu, C.; Wen, Y.; Gao, Y.; Xing, X.; Wei, Z.; Sun, H.; Zhang, Y.-W.; Song, W. Mesoporous Hollow Cu-Ni Alloy Nanocage from Core-Shell Cu@Ni Nanocube for Efficient Hydrogen Evolution Reaction. ACS Catal. 2019, 9, 5084-5095.

(114) McEnaney, J. M.; Soucy, T. L.; Hodges, J. M.; Callejas, J. F.; Mondschein, J. S.; Schaak, R. E. Colloidally-Synthesized Cobalt Molybdenum Nanoparticles as Active and Stable Electrocatalysts for the Hydrogen Evolution Reaction under Alkaline Conditions. $J$. Mater. Chem. A 2016, 4, 3077-3081.

(115) Wang, X.; Zhu, Y.; Vasileff, A.; Jiao, Y.; Chen, S.; Song, L.; Zheng, B.; Zheng, Y.; Qiao, S.-Z. Strain Effect in Bimetallic Electrocatalysts in the Hydrogen Evolution Reaction. ACS Energy Lett. 2018, 3, 1198-1204.

(116) Liu, P.; Rodriguez, J. A. Catalysts for Hydrogen Evolution from the [NiFe] Hydrogenase to the Ni 2 P(001) Surface: The Importance of Ensemble Effect. J. Am. Chem. Soc. 2005, 127, 14871-14878.

(117) Callejas, J. F.; Read, C. G.; Popczun, E. J.; McEnaney, J. M.; Schaak, R. E. Nanostructured Co2P Electrocatalyst for the Hydrogen Evolution Reaction and Direct Comparison with Morphologically Equivalent CoP. Chem. Mater. 2015, 27, 3769-3774.

(118) Popczun, E. J.; McKone, J. R.; Read, C. G.; Biacchi, A. J.; Wiltrout, A. M.; Lewis, N. S.; Schaak, R. E. Nanostructured Nickel Phosphide as an Electrocatalyst for the Hydrogen Evolution Reaction. J. Am. Chem. Soc. 2013, 135, 9267-9270.

(119) Callejas, J. F.; McEnaney, J. M.; Read, C. G.; Crompton, J. C.; Biacchi, A. J.; Popczun, E. J.; Gordon, T. R.; Lewis, N. S.; Schaak, R. E. Electrocatalytic and Photocatalytic Hydrogen Production from Acidic and Neutral-PH Aqueous Solutions Using Iron Phosphide Nanoparticles. ACS Nano 2014, 8, 11101-11107.

(120) McEnaney, J. M.; Crompton, J. C.; Callejas, J. F.; Popczun, E. J.; Biacchi, A. J.; Lewis, N. S.; Schaak, R. E. Amorphous Molybdenum Phosphide Nanoparticles for Electrocatalytic Hydrogen Evolution. Chem. Mater. 2014, 26, 4826-4831.

(121) McEnaney, J. M.; Chance Crompton, J.; Callejas, J. F.; Popczun, E. J.; Read, C. G.; Lewis, N. S.; Schaak, R. E. Electrocatalytic Hydrogen Evolution Using Amorphous Tungsten Phosphide Nanoparticles. Chem. Commun. 2014, 50, 11026.

(122) Seo, B.; Baek, D. S.; Sa, Y. J.; Joo, S. H. Shape Effects of Nickel Phosphide 
Nanocrystals on Hydrogen Evolution Reaction. CrystEngComm 2016, 18, 6083-6089.

(123) Zhou, M.; Kang, Y.; Huang, K.; Shi, Z.; Xie, R.; Yang, W. Ultra-Small Nickel Phosphide Nanoparticles as a High-Performance Electrocatalyst for the Hydrogen Evolution Reaction. RSC Adv. 2016, 6, 74895-74902.

(124) Popczun, E. J.; Read, C. G.; Roske, C. W.; Lewis, N. S.; Schaak, R. E. Highly Active Electrocatalysis of the Hydrogen Evolution Reaction by Cobalt Phosphide Nanoparticles. Angew. Chemie 2014, 126, 5531-5534.

(125) Popczun, E. J.; Roske, C. W.; Read, C. G.; Crompton, J. C.; McEnaney, J. M.; Callejas, J. F.; Lewis, N. S.; Schaak, R. E. Highly Branched Cobalt Phosphide Nanostructures for Hydrogen-Evolution Electrocatalysis. J. Mater. Chem. A 2015, 3, 5420-5425.

(126) Ha, D.-H.; Han, B.; Risch, M.; Giordano, L.; Yao, K. P. C.; Karayaylali, P.; Shao-Horn, Y. Activity and Stability of Cobalt Phosphides for Hydrogen Evolution upon Water Splitting. Nano Energy 2016, 29, 37-45.

(127) Najafi, L.; Bellani, S.; Castelli, A.; Arciniegas, M. P.; Brescia, R.; Oropesa-Nuñez, R.; Martín-García, B.; Serri, M.; Drago, F.; Manna, L.; Bonaccorso, F. Octapod-Shaped CdSe Nanocrystals Hosting Pt with High Mass Activity for the Hydrogen Evolution Reaction. Chem. Mater. 2020, 32, 2420-2429.

(128) Wang, P.; Zhang, X.; Zhang, J.; Wan, S.; Guo, S.; Lu, G.; Yao, J.; Huang, X. Precise Tuning in Platinum-Nickel/Nickel Sulfide Interface Nanowires for Synergistic Hydrogen Evolution Catalysis. Nat. Commun. 2017, 8, 14580.

(129) Maiti, A.; Srivastava, S. K. N, Ru Codoped Pellet Drum Bundle-Like Sb 2 S 3 : An Efficient Hydrogen Evolution Reaction and Hydrogen Oxidation Reaction Electrocatalyst in Alkaline Medium. ACS Appl. Mater. Interfaces 2020, 12, 7057-7070.

(130) Benck, J. D.; Hellstern, T. R.; Kibsgaard, J.; Chakthranont, P.; Jaramillo, T. F. Catalyzing the Hydrogen Evolution Reaction (HER) with Molybdenum Sulfide Nanomaterials. ACS Catal. 2014, 4, 3957-3971.

(131) Yan, Y.; Xia, B.; Xu, Z.; Wang, X. Recent Development of Molybdenum Sulfides as Advanced Electrocatalysts for Hydrogen Evolution Reaction. ACS Catal. 2014, 4, $1693-$ 1705 .

(132) Wang, M.; Dang, Z.; Prato, M.; Petralanda, U.; Infante, I.; Shinde, D. V.; De Trizio, L.; Manna, L. Ruthenium-Decorated Cobalt Selenide Nanocrystals for Hydrogen Evolution. ACS Appl. Nano Mater. 2019, 2, 5695-5703.

(133) Hong, W. T.; Risch, M.; Stoerzinger, K. A.; Grimaud, A.; Suntivich, J.; Shao-Horn, Y. Toward the Rational Design of Non-Precious Transition Metal Oxides for Oxygen 
Electrocatalysis. Energy Environ. Sci. 2015, 8, 1404-1427.

(134) Lewis, N. S.; Nocera, D. G. Powering the Planet: Chemical Challenges in Solar Energy Utilization. Proc. Natl. Acad. Sci. 2006, 103, 15729-15735.

(135) Cook, T. R.; Dogutan, D. K.; Reece, S. Y.; Surendranath, Y.; Teets, T. S.; Nocera, D. G. Solar Energy Supply and Storage for the Legacy and Nonlegacy Worlds. Chem. Rev. 2010, 110, 6474-6502.

(136) McCrory, C. C. L.; Jung, S.; Peters, J. C.; Jaramillo, T. F. Benchmarking Heterogeneous Electrocatalysts for the Oxygen Evolution Reaction. J. Am. Chem. Soc. 2013, 135, $16977-16987$.

(137) Gasteiger, H. A.; Kocha, S. S.; Sompalli, B.; Wagner, F. T. Activity Benchmarks and Requirements for Pt, Pt-Alloy, and Non-Pt Oxygen Reduction Catalysts for PEMFCs. Appl. Catal. B Environ. 2005, 56, 9-35.

(138) Huang, Z.-F.; Wang, J.; Peng, Y.; Jung, C.-Y.; Fisher, A.; Wang, X. Design of Efficient Bifunctional Oxygen Reduction/Evolution Electrocatalyst: Recent Advances and Perspectives. Adv. Energy Mater. 2017, 7, 1700544.

(139) Ma, R.; Lin, G.; Zhou, Y.; Liu, Q.; Zhang, T.; Shan, G.; Yang, M.; Wang, J. A Review of Oxygen Reduction Mechanisms for Metal-Free Carbon-Based Electrocatalysts. npj Comput. Mater. 2019, 5, 78.

(140) McCrory, C. C. L.; Jung, S.; Ferrer, I. M.; Chatman, S. M.; Peters, J. C.; Jaramillo, T. F. Benchmarking Hydrogen Evolving Reaction and Oxygen Evolving Reaction Electrocatalysts for Solar Water Splitting Devices. J. Am. Chem. Soc. 2015, 137, 43474357.

(141) Fabbri, E.; Schmidt, T. J. Oxygen Evolution Reaction-The Enigma in Water Electrolysis. ACS Catal. 2018, 8, 9765-9774.

(142) Oh, H.-S.; Nong, H. N.; Reier, T.; Gliech, M.; Strasser, P. Oxide-Supported Ir Nanodendrites with High Activity and Durability for the Oxygen Evolution Reaction in Acid PEM Water Electrolyzers. Chem. Sci. 2015, 6, 3321-3328.

(143) Quinson, J.; Neumann, S.; Wannmacher, T.; Kacenauskaite, L.; Inaba, M.; Bucher, J.; Bizzotto, F.; Simonsen, S. B.; Theil Kuhn, L.; Bujak, D.; Zana, A.; Arenz, M.; Kunz, S. Colloids for Catalysts: A Concept for the Preparation of Superior Catalysts of Industrial Relevance. Angew. Chemie Int. Ed. 2018, 57, 12338-12341.

(144) Arminio-Ravelo, J. A.; Quinson, J.; Pedersen, M. A.; Kirkensgaard, J. J. K.; Arenz, M.; Escudero-Escribano, M. Synthesis of Iridium Nanocatalysts for Water Oxidation in Acid: Effect of the Surfactant. ChemCatChem 2020, 12, 1282-1287. 
(145) Bizzotto, F.; Quinson, J.; Zana, A.; Kirkensgaard, J. J. K.; Dworzak, A.; Oezaslan, M.; Arenz, M. Ir Nanoparticles with Ultrahigh Dispersion as Oxygen Evolution Reaction (OER) Catalysts: Synthesis and Activity Benchmarking. Catal. Sci. Technol. 2019, 9, 6345-6356.

(146) Abbott, D. F.; Lebedev, D.; Waltar, K.; Povia, M.; Nachtegaal, M.; Fabbri, E.; Copéret, C.; Schmidt, T. J. Iridium Oxide for the Oxygen Evolution Reaction: Correlation between Particle Size, Morphology, and the Surface Hydroxo Layer from Operando XAS. Chem. Mater. 2016, 28, 6591-6604.

(147) Zhu, H.; Zhang, S.; Huang, Y.-X.; Wu, L.; Sun, S. Monodisperse M x Fe 3- x O 4 (M $=\mathrm{Fe}, \mathrm{Cu}, \mathrm{Co}, \mathrm{Mn}$ ) Nanoparticles and Their Electrocatalysis for Oxygen Reduction Reaction. Nano Lett. 2013, 13, 2947-2951.

(148) Samira, S.; Gu, X.-K.; Nikolla, E. Design Strategies for Efficient Nonstoichiometric Mixed Metal Oxide Electrocatalysts: Correlating Measurable Oxide Properties to Electrocatalytic Performance. ACS Catal. 2019, 9, 10575-10586.

(149) Carneiro, J. S. A.; Williams, J.; Gryko, A.; Herrera, L. P.; Nikolla, E. Embracing the Complexity of Catalytic Structures: A Viewpoint on the Synthesis of Nonstoichiometric Mixed Metal Oxides for Catalysis. ACS Catal. 2020, 10, 516-527.

(150) Nørskov, J. K.; Rossmeis1, J.; Logadottir, A.; Lindqvist, L.; Kitchin, J. R.; Bligaard, T.; Jónsson, H. Origin of the Overpotential for Oxygen Reduction at a Fuel-Cell Cathode. J. Phys. Chem. B 2004, 108, 17886-17892.

(151) Marković, N. M.; Gasteiger, H. A.; Ross, P. N. Oxygen Reduction on Platinum LowIndex Single-Crystal Surfaces in Alkaline Solution: Rotating Ring Disk Pt( Hkl ) Studies. J. Phys. Chem. 1996, 100, 6715-6721.

(152) Marković, N. M.; Adžić, R. R.; Cahan, B. D.; Yeager, E. B. Structural Effects in Electrocatalysis: Oxygen Reduction on Platinum Low Index Single-Crystal Surfaces in Perchloric Acid Solutions. J. Electroanal. Chem. 1994, 377, 249-259.

(153) Stamenkovic, V. R.; Fowler, B.; Mun, B. S.; Wang, G.; Ross, P. N.; Lucas, C. A.; Markovic, N. M. Improved Oxygen Reduction Activity on Pt3Ni(111) via Increased Surface Site Availability. Science 2007, 315, 493-497.

(154) Sui, S.; Wang, X.; Zhou, X.; Su, Y.; Riffat, S.; Liu, C. A Comprehensive Review of Pt Electrocatalysts for the Oxygen Reduction Reaction: Nanostructure, Activity, Mechanism and Carbon Support in PEM Fuel Cells. J. Mater. Chem. A 2017, 5, 18081825 .

(155) Chen, J.; Lim, B.; Lee, E. P.; Xia, Y. Shape-Controlled Synthesis of Platinum 
Nanocrystals for Catalytic and Electrocatalytic Applications. Nano Today 2009, 4, 8195.

(156) Lv, H.; Li, D.; Strmcnik, D.; Paulikas, A. P.; Markovic, N. M.; Stamenkovic, V. R. Recent Advances in the Design of Tailored Nanomaterials for Efficient Oxygen Reduction Reaction. Nano Energy 2016, 29, 149-165.

(157) Yu, T.; Kim, D. Y.; Zhang, H.; Xia, Y. Platinum Concave Nanocubes with High-Index Facets and Their Enhanced Activity for Oxygen Reduction Reaction. Angew. Chemie Int. Ed. 2011, 50, 2773-2777.

(158) Chen, R.; Cao, Z.; Lyu, Z.; Xie, M.; Shi, Y.; Xia, Y. Continuous and Scalable Synthesis of Pt Multipods with Enhanced Electrocatalytic Activity toward the Oxygen Reduction Reaction. ChemNanoMat 2019, 5, 599-605.

(159) Wang, C.; Daimon, H.; Onodera, T.; Koda, T.; Sun, S. A General Approach to the Sizeand Shape-Controlled Synthesis of Platinum Nanoparticles and Their Catalytic Reduction of Oxygen. Angew. Chemie Int. Ed. 2008, 47, 3588-3591.

(160) Wang, C.; Daimon, H.; Lee, Y.; Kim, J.; Sun, S. Synthesis of Monodisperse Pt Nanocubes and Their Enhanced Catalysis for Oxygen Reduction. J. Am. Chem. Soc. 2007, 129, 6974-6975.

(161) He, D. S.; He, D.; Wang, J.; Lin, Y.; Yin, P.; Hong, X.; Wu, Y.; Li, Y. Ultrathin Icosahedral Pt-Enriched Nanocage with Excellent Oxygen Reduction Reaction Activity. J. Am. Chem. Soc. 2016, 138, 1494-1497.

(162) Wang, X.; Figueroa-Cosme, L.; Yang, X.; Luo, M.; Liu, J.; Xie, Z.; Xia, Y. Pt-Based Icosahedral Nanocages: Using a Combination of $\{111\}$ Facets, Twin Defects, and Ultrathin Walls to Greatly Enhance Their Activity toward Oxygen Reduction. Nano Lett. 2016, 16, 1467-1471.

(163) Li, D.; Wang, C.; Strmcnik, D. S.; Tripkovic, D. V.; Sun, X.; Kang, Y.; Chi, M.; Snyder, J. D.; van der Vliet, D.; Tsai, Y.; Stamenkovic, V. R.; Sun, S.; Markovic, N. M. Functional Links between Pt Single Crystal Morphology and Nanoparticles with Different Size and Shape: The Oxygen Reduction Reaction Case. Energy Environ. Sci. 2014, 7, 4061-4069.

(164) Shao, M.; Yu, T.; Odell, J. H.; Jin, M.; Xia, Y. Structural Dependence of Oxygen Reduction Reaction on Palladium Nanocrystals. Chem. Commun. 2011, 47, 6566.

(165) Shao, M. Palladium-Based Electrocatalysts for Hydrogen Oxidation and Oxygen Reduction Reactions. J. Power Sources 2011, 196, 2433-2444.

(166) Zhang, H.; Jin, M.; Xiong, Y.; Lim, B.; Xia, Y. Shape-Controlled Synthesis of Pd 
Nanocrystals and Their Catalytic Applications. Acc. Chem. Res. 2013, 46, 1783-1794.

(167) Wang, H.; Niu, G.; Zhou, M.; Wang, X.; Park, J.; Bao, S.; Chi, M.; Cai, Z.; Xia, Y. Scalable Synthesis of Palladium Icosahedra in Plug Reactors for the Production of Oxygen Reduction Reaction Catalysts. ChemCatChem 2016, 8, 1658-1664.

(168) Stamenkovic, V. R.; Mun, B. S.; Arenz, M.; Mayrhofer, K. J. J.; Lucas, C. A.; Wang, G.; Ross, P. N.; Markovic, N. M. Trends in Electrocatalysis on Extended and Nanoscale Pt-Bimetallic Alloy Surfaces. Nat. Mater. 2007, 6, 241-247.

(169) Cui, C.; Gan, L.; Heggen, M.; Rudi, S.; Strasser, P. Compositional Segregation in Shaped Pt Alloy Nanoparticles and Their Structural Behaviour during Electrocatalysis. Nat. Mater. 2013, 12, 765-771.

(170) Chen, C.; Kang, Y.; Huo, Z.; Zhu, Z.; Huang, W.; Xin, H. L.; Snyder, J. D.; Li, D.; Herron, J. A.; Mavrikakis, M.; Chi, M.; More, K. L.; Li, Y.; Markovic, N. M.; Somorjai, G. A.; Yang, P.; Stamenkovic, V. R. Highly Crystalline Multimetallic Nanoframes with Three-Dimensional Electrocatalytic Surfaces. Science 2014, 343, 1339-1343.

(171) Choi, S.-I.; Xie, S.; Shao, M.; Odell, J. H.; Lu, N.; Peng, H.-C.; Protsailo, L.; Guerrero, S.; Park, J.; Xia, X.; Wang, J.; Kim, M. J.; Xia, Y. Synthesis and Characterization of 9 $\mathrm{Nm}$ Pt-Ni Octahedra with a Record High Activity of $3.3 \mathrm{~A} / \mathrm{Mg}$ Pt for the Oxygen Reduction Reaction. Nano Lett. 2013, 13, 3420-3425.

(172) Niu, G.; Zhou, M.; Yang, X.; Park, J.; Lu, N.; Wang, J.; Kim, M. J.; Wang, L.; Xia, Y. Synthesis of Pt-Ni Octahedra in Continuous-Flow Droplet Reactors for the Scalable Production of Highly Active Catalysts toward Oxygen Reduction. Nano Lett. 2016, 16, $3850-3857$.

(173) Wu, J.; Zhang, J.; Peng, Z.; Yang, S.; Wagner, F. T.; Yang, H. Truncated Octahedral Pt3Ni Oxygen Reduction Reaction Electrocatalysts. J. Am. Chem. Soc. 2010, 132, 49844985.

(174) Wang, H.; Zhou, S.; Gilroy, K. D.; Cai, Z.; Xia, Y. Icosahedral Nanocrystals of Noble Metals: Synthesis and Applications. Nano Today 2017, 15, 121-144.

(175) Zhao, M.; Wang, X.; Yang, X.; Gilroy, K. D.; Qin, D.; Xia, Y. Hollow Metal Nanocrystals with Ultrathin, Porous Walls and Well-Controlled Surface Structures. $A d v$. Mater. 2018, 30, 1801956.

(176) Wu, J.; Qi, L.; You, H.; Gross, A.; Li, J.; Yang, H. Icosahedral Platinum Alloy Nanocrystals with Enhanced Electrocatalytic Activities. J. Am. Chem. Soc. 2012, 134, $11880-11883$.

(177) Zhang, J.; Yang, H.; Fang, J.; Zou, S. Synthesis and Oxygen Reduction Activity of 
Shape-Controlled Pt3Ni Nanopolyhedra. Nano Lett. 2010, 10, 638-644.

(178) Choi, K.-H.; Jang, Y.; Chung, D. Y.; Seo, P.; Jun, S. W.; Lee, J. E.; Oh, M. H.; Shokouhimehr, M.; Jung, N.; Yoo, S. J.; Sung, Y.-E.; Hyeon, T. A Simple Synthesis of Urchin-like Pt-Ni Bimetallic Nanostructures as Enhanced Electrocatalysts for the Oxygen Reduction Reaction. Chem. Commun. 2016, 52, 597-600.

(179) Huang, X.; Zhao, Z.; Cao, L.; Chen, Y.; Zhu, E.; Lin, Z.; Li, M.; Yan, A.; Zettl, A.; Wang, Y. M.; Duan, X.; Mueller, T.; Huang, Y. High-Performance Transition MetalDoped Pt3Ni Octahedra for Oxygen Reduction Reaction. Science 2015, 348, 12301234.

(180) Becknell, N.; Kang, Y.; Chen, C.; Resasco, J.; Kornienko, N.; Guo, J.; Markovic, N. M.; Somorjai, G. A.; Stamenkovic, V. R.; Yang, P. Atomic Structure of Pt 3 Ni Nanoframe Electrocatalysts by in Situ X-Ray Absorption Spectroscopy. J. Am. Chem. Soc. 2015, 137, 15817-15824.

(181) Becknell, N.; Son, Y.; Kim, D.; Li, D.; Yu, Y.; Niu, Z.; Lei, T.; Sneed, B. T.; More, K. L.; Markovic, N. M.; Stamenkovic, V. R.; Yang, P. Control of Architecture in Rhombic Dodecahedral Pt-Ni Nanoframe Electrocatalysts. J. Am. Chem. Soc. 2017, 139, 1167811681.

(182) Chen, S.; Niu, Z.; Xie, C.; Gao, M.; Lai, M.; Li, M.; Yang, P. Effects of Catalyst Processing on the Activity and Stability of Pt-Ni Nanoframe Electrocatalysts. ACS Nano 2018, 12, 8697-8705.

(183) Lee, J. D.; Jishkariani, D.; Zhao, Y.; Najmr, S.; Rosen, D.; Kikkawa, J. M.; Stach, E. A.; Murray, C. B. Tuning the Electrocatalytic Oxygen Reduction Reaction Activity of PtCo Nanocrystals by Cobalt Concentration with Atomic-Scale Understanding. ACS Appl. Mater. Interfaces 2019, 11, 26789-26797.

(184) Wang, D.; Xin, H. L.; Hovden, R.; Wang, H.; Yu, Y.; Muller, D. A.; DiSalvo, F. J.; Abruña, H. D. Structurally Ordered Intermetallic Platinum-Cobalt Core-Shell Nanoparticles with Enhanced Activity and Stability as Oxygen Reduction Electrocatalysts. Nat. Mater. 2013, 12, 81-87.

(185) Xiong, Y.; Xiao, L.; Yang, Y.; DiSalvo, F. J.; Abruña, H. D. High-Loading Intermetallic Pt $3 \mathrm{Co} / \mathrm{C}$ Core-Shell Nanoparticles as Enhanced Activity Electrocatalysts toward the Oxygen Reduction Reaction (ORR). Chem. Mater. 2018, 30, 1532-1539.

(186) Stamenkovic, V. R.; Mun, B. S.; Mayrhofer, K. J. J.; Ross, P. N.; Markovic, N. M. Effect of Surface Composition on Electronic Structure, Stability, and Electrocatalytic Properties of Pt-Transition Metal Alloys: Pt-Skin versus Pt-Skeleton Surfaces. J. Am. 
Chem. Soc. 2006, 128, 8813-8819.

(187) Chen, S.; Li, M.; Gao, M.; Jin, J.; van Spronsen, M. A.; Salmeron, M. B.; Yang, P. HighPerformance Pt-Co Nanoframes for Fuel-Cell Electrocatalysis. Nano Lett. 2020, 20, 1974-1979.

(188) Kim, J.; Lee, Y.; Sun, S. Structurally Ordered FePt Nanoparticles and Their Enhanced Catalysis for Oxygen Reduction Reaction. J. Am. Chem. Soc. 2010, 132, 4996-4997.

(189) Li, Q.; Wu, L.; Wu, G.; Su, D.; Lv, H.; Zhang, S.; Zhu, W.; Casimir, A.; Zhu, H.; Mendoza-Garcia, A.; Sun, S. New Approach to Fully Ordered Fct-FePt Nanoparticles for Much Enhanced Electrocatalysis in Acid. Nano Lett. 2015, 15, 2468-2473.

(190) Chung, D. Y.; Jun, S. W.; Yoon, G.; Kwon, S. G.; Shin, D. Y.; Seo, P.; Yoo, J. M.; Shin, H.; Chung, Y.-H.; Kim, H.; Mun, B. S.; Lee, K.-S.; Lee, N.-S.; Yoo, S. J.; Lim, D.-H.; Kang, K.; Sung, Y.-E.; Hyeon, T. Highly Durable and Active PtFe Nanocatalyst for Electrochemical Oxygen Reduction Reaction. J. Am. Chem. Soc. 2015, 137, 1547815485 .

(191) Li, J.; Xi, Z.; Pan, Y.-T.; Spendelow, J. S.; Duchesne, P. N.; Su, D.; Li, Q.; Yu, C.; Yin, Z.; Shen, B.; Kim, Y. S.; Zhang, P.; Sun, S. Fe Stabilization by Intermetallic L1 0 -FePt and Pt Catalysis Enhancement in L1 0 -FePt/Pt Nanoparticles for Efficient Oxygen Reduction Reaction in Fuel Cells. J. Am. Chem. Soc. 2018, 140, 2926-2932.

(192) Guo, S.; Li, D.; Zhu, H.; Zhang, S.; Markovic, N. M.; Stamenkovic, V. R.; Sun, S. FePt and CoPt Nanowires as Efficient Catalysts for the Oxygen Reduction Reaction. Angew. Chemie Int. Ed. 2013, 52, 3465-3468.

(193) Zhu, J.; Elnabawy, A. O.; Lyu, Z.; Xie, M.; Murray, E. A.; Chen, Z.; Jin, W.; Mavrikakis, M.; Xia, Y. Facet-Controlled Pt-Ir Nanocrystals with Substantially Enhanced Activity and Durability towards Oxygen Reduction. Mater. Today 2020, 35, 69-77.

(194) Zhu, J.; Xie, M.; Chen, Z.; Lyu, Z.; Chi, M.; Jin, W.; Xia, Y. Pt-Ir-Pd Trimetallic Nanocages as a Dual Catalyst for Efficient Oxygen Reduction and Evolution Reactions in Acidic Media. Adv. Energy Mater. 2020, 10, 1904114.

(195) Jiang, G.; Zhu, H.; Zhang, X.; Shen, B.; Wu, L.; Zhang, S.; Lu, G.; Wu, Z.; Sun, S. Core/Shell Face-Centered Tetragonal FePd/Pd Nanoparticles as an Efficient Non-Pt Catalyst for the Oxygen Reduction Reaction. ACS Nano 2015, 9, 11014-11022.

(196) Kariuki, N. N.; Wang, X.; Mawdsley, J. R.; Ferrandon, M. S.; Niyogi, S. G.; Vaughey, J. T.; Myers, D. J. Colloidal Synthesis and Characterization of Carbon-Supported Pd-Cu Nanoparticle Oxygen Reduction Electrocatalysts. Chem. Mater. 2010, 22, 4144-4152.

(197) Liu, S.; Zhang, Q.; Li, Y.; Han, M.; Gu, L.; Nan, C.; Bao, J.; Dai, Z. Five-Fold Twinned 
Pd 2 NiAg Nanocrystals with Increased Surface Ni Site Availability to Improve Oxygen Reduction Activity. J. Am. Chem. Soc. 2015, 137, 2820-2823.

(198) Jiang, K.; Wang, P.; Guo, S.; Zhang, X.; Shen, X.; Lu, G.; Su, D.; Huang, X. Ordered $\mathrm{PdCu}$-Based Nanoparticles as Bifunctional Oxygen-Reduction and Ethanol-Oxidation Electrocatalysts. Angew. Chemie Int. Ed. 2016, 55, 9030-9035.

(199) Nitopi, S.; Bertheussen, E.; Scott, S. B.; Liu, X.; Engstfeld, A. K.; Horch, S.; Seger, B.; Stephens, I. E. L.; Chan, K.; Hahn, C.; Nørskov, J. K.; Jaramillo, T. F.; Chorkendorff, I. Progress and Perspectives of Electrochemical CO2 Reduction on Copper in Aqueous Electrolyte. Chem. Rev. 2019, 119, 7610-7672.

(200) Gao, D.; Arán-Ais, R. M.; Jeon, H. S.; Roldan Cuenya, B. Rational Catalyst and Electrolyte Design for CO2 Electroreduction towards Multicarbon Products. Nat. Catal. 2019, 2, 198-210.

(201) Liu, X.; Xiao, J.; Peng, H.; Hong, X.; Chan, K.; Nørskov, J. K. Understanding Trends in Electrochemical Carbon Dioxide Reduction Rates. Nat. Commun. 2017, 8, 15438.

(202) Hori, Y.; Takahashi, I.; Koga, O.; Hoshi, N. Selective Formation of C2 Compounds from Electrochemical Reduction of $\mathrm{CO} 2$ at a Series of Copper Single Crystal Electrodes. $J$. Phys. Chem. B 2002, 106, 15-17.

(203) Hori, Y.; Takahashi, I.; Koga, O.; Hoshi, N. Electrochemical Reduction of Carbon Dioxide at Various Series of Copper Single Crystal Electrodes. J. Mol. Catal. A Chem. 2003, 199, 39-47.

(204) Hahn, C.; Hatsukade, T.; Kim, Y.-G.; Vailionis, A.; Baricuatro, J. H.; Higgins, D. C.; Nitopi, S. A.; Soriaga, M. P.; Jaramillo, T. F. Engineering Cu Surfaces for the Electrocatalytic Conversion of CO2: Controlling Selectivity toward Oxygenates and Hydrocarbons. Proc. Natl. Acad. Sci. 2017, 114, 5918-5923.

(205) Hong, X.; Chan, K.; Tsai, C.; Nørskov, J. K. How Doped MoS2 Breaks Transition-Metal Scaling Relations for CO2 Electrochemical Reduction. ACS Catal. 2016, 6, 4428-4437.

(206) Li, Y. C.; Wang, Z.; Yuan, T.; Nam, D.-H.; Luo, M.; Wicks, J.; Chen, B.; Li, J.; Li, F.; de Arquer, F. P. G.; Wang, Y.; Dinh, C.-T.; Voznyy, O.; Sinton, D.; Sargent, E. H. Binding Site Diversity Promotes CO2 Electroreduction to Ethanol. J. Am. Chem. Soc. 2019, 141, 8584-8591.

(207) Gao, D.; Zhou, H.; Wang, J.; Miao, S.; Yang, F.; Wang, G.; Wang, J.; Bao, X. SizeDependent Electrocatalytic Reduction of $\mathrm{CO} 2$ over Pd Nanoparticles. J. Am. Chem. Soc. 2015, 137, 4288-4291.

(208) Klinkova, A.; De Luna, P.; Dinh, C.-T.; Voznyy, O.; Larin, E. M.; Kumacheva, E.; 
Sargent, E. H. Rational Design of Efficient Palladium Catalysts for Electroreduction of Carbon Dioxide to Formate. ACS Catal. 2016, 6, 8115-8120.

(209) Zhang, S.; Kang, P.; Meyer, T. J. Nanostructured Tin Catalysts for Selective Electrochemical Reduction of Carbon Dioxide to Formate. J. Am. Chem. Soc. 2014, 136, 1734-1737.

(210) Jeon, H. S.; Sinev, I.; Scholten, F.; Divins, N. J.; Zegkinoglou, I.; Pielsticker, L.; Roldan Cuenya, B. Operando Evolution of the Structure and Oxidation State of Size-Controlled Zn Nanoparticles during CO2 Electroreduction. J. Am. Chem. Soc. 2018, 140, $9383-$ 9386.

(211) Zhu, W.; Michalsky, R.; Metin, Ö.; Lv, H.; Guo, S.; Wright, C. J.; Sun, X.; Peterson, A. A.; Sun, S. Monodisperse Au Nanoparticles for Selective Electrocatalytic Reduction of CO2 to CO. J. Am. Chem. Soc. 2013, 135, 16833-16836.

(212) Zhu, W.; Zhang, Y.-J.; Zhang, H.; Lv, H.; Li, Q.; Michalsky, R.; Peterson, A. A.; Sun, S. Active and Selective Conversion of $\mathrm{CO} 2$ to $\mathrm{CO}$ on Ultrathin Au Nanowires. J. Am. Chem. Soc. 2014, 136, 16132-16135.

(213) Mistry, H.; Reske, R.; Zeng, Z.; Zhao, Z.-J.; Greeley, J.; Strasser, P.; Roldan Cuenya, B. Exceptional Size-Dependent Activity Enhancement in the Electroreduction of CO2 over Au Nanoparticles. J. Am. Chem. Soc. 2014, 136, 16473-16476.

(214) Mezzavilla, S.; Horch, S.; Stephens, I. E. L.; Seger, B.; Chorkendorff, I. Structure Sensitivity in the Electrocatalytic Reduction of $\mathrm{CO} 2$ with Gold Catalysts. Angew. Chemie Int. Ed. 2019, 58, 3774-3778.

(215) Lee, H.-E.; Yang, K. D.; Yoon, S. M.; Ahn, H.-Y.; Lee, Y. Y.; Chang, H.; Jeong, D. H.; Lee, Y.-S.; Kim, M. Y.; Nam, K. T. Concave Rhombic Dodecahedral Au Nanocatalyst with Multiple High-Index Facets for CO2 Reduction. ACS Nano 2015, 9, 8384-8393.

(216) Liu, S.; Tao, H.; Zeng, L.; Liu, Q.; Xu, Z.; Liu, Q.; Luo, J.-L. Shape-Dependent Electrocatalytic Reduction of CO2 to CO on Triangular Silver Nanoplates. J. Am. Chem. Soc. 2017, 139, 2160-2163.

(217) Manthiram, K.; Beberwyck, B. J.; Alivisatos, A. P. Enhanced Electrochemical Methanation of Carbon Dioxide with a Dispersible Nanoscale Copper Catalyst. J. Am. Chem. Soc. 2014, 136, 13319-13325.

(218) Reske, R.; Mistry, H.; Behafarid, F.; Roldan Cuenya, B.; Strasser, P. Particle Size Effects in the Catalytic Electroreduction of $\mathrm{CO} 2$ on Cu Nanoparticles. J. Am. Chem. Soc. 2014, 136, 6978-6986.

(219) Loiudice, A.; Lobaccaro, P.; Kamali, E. A.; Thao, T.; Huang, B. H.; Ager, J. W.; 
Buonsanti, R. Tailoring Copper Nanocrystals towards C2 Products in Electrochemical CO2 Reduction. Angew. Chemie Int. Ed. 2016, 55, 5789-5792.

(220) Mangione, G.; Huang, J.; Buonsanti, R.; Corminboeuf, C. Dual-Facet Mechanism in Copper Nanocubes for Electrochemical CO2 Reduction into Ethylene. J. Phys. Chem. Lett. 2019, 10, 4259-4265.

(221) Kim, D.; Kley, C. S.; Li, Y.; Yang, P. Copper Nanoparticle Ensembles for Selective Electroreduction of CO2 to C2 -C3 Products. Proc. Natl. Acad. Sci. 2017, 114, 1056010565 .

(222) Li, Y.; Cui, F.; Ross, M. B.; Kim, D.; Sun, Y.; Yang, P. Structure-Sensitive CO2 Electroreduction to Hydrocarbons on Ultrathin 5-Fold Twinned Copper Nanowires. Nano Lett. 2017, 17, 1312-1317.

(223) Suen, N.-T.; Kong, Z.-R.; Hsu, C.-S.; Chen, H.-C.; Tung, C.-W.; Lu, Y.-R.; Dong, C.L.; Shen, C.-C.; Chung, J.-C.; Chen, H. M. Morphology Manipulation of Copper Nanocrystals and Product Selectivity in the Electrocatalytic Reduction of Carbon Dioxide. ACS Catal. 2019, 9, 5217-5222.

(224) Kim, C.; Dionigi, F.; Beermann, V.; Wang, X.; Möller, T.; Strasser, P. Alloy Nanocatalysts for the Electrochemical Oxygen Reduction (ORR) and the Direct Electrochemical Carbon Dioxide Reduction Reaction (CO2RR). Adv. Mater. 2019, 31, 1805617.

(225) Kim, D.; Xie, C.; Becknell, N.; Yu, Y.; Karamad, M.; Chan, K.; Crumlin, E. J.; Nørskov, J. K.; Yang, P. Electrochemical Activation of CO2 through Atomic Ordering Transformations of AuCu Nanoparticles. J. Am. Chem. Soc. 2017, 139, 8329-8336.

(226) Zhu, W.; Zhang, L.; Yang, P.; Hu, C.; Dong, H.; Zhao, Z.-J.; Mu, R.; Gong, J. Formation of Enriched Vacancies for Enhanced CO2 Electrocatalytic Reduction over AuCu Alloys. ACS Energy Lett. 2018, 3, 2144-2149.

(227) Tao, Z.; Wu, Z.; Yuan, X.; Wu, Y.; Wang, H. Copper-Gold Interactions Enhancing Formate Production from Electrochemical CO2 Reduction. ACS Catal. 2019, 9, 1089410898.

(228) Clark, E. L.; Hahn, C.; Jaramillo, T. F.; Bell, A. T. Electrochemical CO2 Reduction over Compressively Strained CuAg Surface Alloys with Enhanced Multi-Carbon Oxygenate Selectivity. J. Am. Chem. Soc. 2017, 139, 15848-15857.

(229) Lum, Y.; Ager, J. W. Sequential Catalysis Controls Selectivity in Electrochemical CO2 Reduction on Cu. Energy Environ. Sci. 2018, 11, 2935-2944.

(230) Wang, Z.; Yang, G.; Zhang, Z.; Jin, M.; Yin, Y. Selectivity on Etching: Creation of 
High-Energy Facets on Copper Nanocrystals for CO2 Electrochemical Reduction. ACS Nano 2016, 10, 4559-4564.

(231) Ma, S.; Sadakiyo, M.; Heima, M.; Luo, R.; Haasch, R. T.; Gold, J. I.; Yamauchi, M.; Kenis, P. J. A. Electroreduction of Carbon Dioxide to Hydrocarbons Using Bimetallic $\mathrm{Cu}-\mathrm{Pd}$ Catalysts with Different Mixing Patterns. J. Am. Chem. Soc. 2017, 139, 47-50.

(232) Ong, B. C.; Kamarudin, S. K.; Basri, S. Direct Liquid Fuel Cells: A Review. Int. J. Hydrogen Energy 2017, 42, 10142-10157.

(233) Siwal, S. S.; Thakur, S.; Zhang, Q. B.; Thakur, V. K. Electrocatalysts for Electrooxidation of Direct Alcohol Fuel Cell: Chemistry and Applications. Mater. Today Chem. 2019, 14, 100182.

(234) Rizo, R.; Sebastián, D.; Rodríguez, J. L.; Lázaro, M. J.; Pastor, E. Influence of the Nature of the Carbon Support on the Activity of Pt/C Catalysts for Ethanol and Carbon Monoxide Oxidation. J. Catal. 2017, 348, 22-28.

(235) Miyake, H.; Okada, T.; Samjeské, G.; Osawa, M. Formic Acid Electrooxidation on Pd in Acidic Solutions Studied by Surface-Enhanced Infrared Absorption Spectroscopy. Phys. Chem. Chem. Phys. 2008, 10, 3662.

(236) Shao, M.; Odell, J.; Humbert, M.; Yu, T.; Xia, Y. Electrocatalysis on Shape-Controlled Palladium Nanocrystals: Oxygen Reduction Reaction and Formic Acid Oxidation. $J$. Phys. Chem. C 2013, 117, 4172-4180.

(237) Xia, X.; Choi, S.-I.; Herron, J. A.; Lu, N.; Scaranto, J.; Peng, H.-C.; Wang, J.; Mavrikakis, M.; Kim, M. J.; Xia, Y. Facile Synthesis of Palladium Right Bipyramids and Their Use as Seeds for Overgrowth and as Catalysts for Formic Acid Oxidation. $J$. Am. Chem. Soc. 2013, 135, 15706-15709.

(238) Wang, Y.; Choi, S.-I.; Zhao, X.; Xie, S.; Peng, H.-C.; Chi, M.; Huang, C. Z.; Xia, Y. Polyol Synthesis of Ultrathin Pd Nanowires via Attachment-Based Growth and Their Enhanced Activity towards Formic Acid Oxidation. Adv. Funct. Mater. 2014, 24, 131139.

(239) Huang, H.; Ruditskiy, A.; Choi, S.-I.; Zhang, L.; Liu, J.; Ye, Z.; Xia, Y. One-Pot Synthesis of Penta-Twinned Palladium Nanowires and Their Enhanced Electrocatalytic Properties. ACS Appl. Mater. Interfaces 2017, 9, 31203-31212.

(240) Lv, T.; Wang, Y.; Choi, S.-I.; Chi, M.; Tao, J.; Pan, L.; Huang, C. Z.; Zhu, Y.; Xia, Y. Controlled Synthesis of Nanosized Palladium Icosahedra and Their Catalytic Activity towards Formic-Acid Oxidation. ChemSusChem 2013, 6, 1923-1930.

(241) Jin, M.; Zhang, H.; Xie, Z.; Xia, Y. Palladium Nanocrystals Enclosed by $\{100\}$ and 
\{111\} Facets in Controlled Proportions and Their Catalytic Activities for Formic Acid Oxidation. Energy Environ. Sci. 2012, 5, 6352-6357.

(242) Jin, M.; Zhang, H.; Xie, Z.; Xia, Y. Palladium Concave Nanocubes with High-Index Facets and Their Enhanced Catalytic Properties. Angew. Chemie Int. Ed. 2011, 50, 7850-7854.

(243) Choi, S.-I.; Herron, J. A.; Scaranto, J.; Huang, H.; Wang, Y.; Xia, X.; Lv, T.; Park, J.; Peng, H.-C.; Mavrikakis, M.; Xia, Y. A Comprehensive Study of Formic Acid Oxidation on Palladium Nanocrystals with Different Types of Facets and Twin Defects. ChemCatChem 2015, 7, 2077-2084.

(244) Xi, Z.; Erdosy, D. P.; Mendoza-Garcia, A.; Duchesne, P. N.; Li, J.; Muzzio, M.; Li, Q.; Zhang, P.; Sun, S. Pd Nanoparticles Coupled to WO 2.72 Nanorods for Enhanced Electrochemical Oxidation of Formic Acid. Nano Lett. 2017, 17, 2727-2731.

(245) Jin, T.; Guo, S.; Zuo, J.; Sun, S. Synthesis and Assembly of Pd Nanoparticles on Graphene for Enhanced Electrooxidation of Formic Acid. Nanoscale 2013, 5, 160-163.

(246) Zheng, W.; Qu, J.; Hong, X.; Tedsree, K.; Tsang, S. C. E. Probing the Size and Shape Effects of Cubic- and Spherical-Shaped Palladium Nanoparticles in the Electrooxidation of Formic Acid. Chem CatChem 2015, 7, 3826-3831.

(247) Huang, X.; Tang, S.; Zhang, H.; Zhou, Z.; Zheng, N. Controlled Formation of Concave Tetrahedral/Trigonal Bipyramidal Palladium Nanocrystals. J. Am. Chem. Soc. 2009, 131, 13916-13917.

(248) Huang, H.; Chen, R.; Liu, M.; Wang, J.; Kim, M. J.; Ye, Z.; Xia, Y. Aqueous Synthesis of $\mathrm{Pd}-\mathrm{M}(\mathrm{M}=\mathrm{Pd}, \mathrm{Pt}$, and $\mathrm{Au})$ Decahedra with Concave Facets for Catalytic Applications. Top. Catal. 2020, 63, 664-672.

(249) Shao, Z.; Zhu, W.; Wang, H.; Yang, Q.; Yang, S.; Liu, X.; Wang, G. Controllable Synthesis of Concave Nanocubes, Right Bipyramids, and 5-Fold Twinned Nanorods of Palladium and Their Enhanced Electrocatalytic Performance. J. Phys. Chem. C 2013, 117, 14289-14294.

(250) Yu, Y.; Zhang, Q.; Liu, B.; Lee, J. Y. Synthesis of Nanocrystals with Variable HighIndex Pd Facets through the Controlled Heteroepitaxial Growth of Trisoctahedral Au Templates. J. Am. Chem. Soc. 2010, 132, 18258-18265.

(251) Zhang, L.; Choi, S.-I.; Tao, J.; Peng, H.-C.; Xie, S.; Zhu, Y.; Xie, Z.; Xia, Y. Pd-Cu Bimetallic Tripods: A Mechanistic Understanding of the Synthesis and Their Enhanced Electrocatalytic Activity for Formic Acid Oxidation. Adv. Funct. Mater. 2014, 24, 7520-7529. 
(252) Ho, S. F.; Mendoza-Garcia, A.; Guo, S.; He, K.; Su, D.; Liu, S.; Metin, Ö.; Sun, S. A Facile Route to Monodisperse MPd $(\mathrm{M}=\mathrm{Co}$ or $\mathrm{Cu})$ Alloy Nanoparticles and Their Catalysis for Electrooxidation of Formic Acid. Nanoscale 2014, 6, 6970-6973.

(253) Mazumder, V.; Chi, M.; Mankin, M. N.; Liu, Y.; Metin, Ö.; Sun, D.; More, K. L.; Sun, S. A Facile Synthesis of MPd $(\mathrm{M}=\mathrm{Co}, \mathrm{Cu})$ Nanoparticles and Their Catalysis for Formic Acid Oxidation. Nano Lett. 2012, 12, 1102-1106.

(254) Wang, C.; Zhang, L.; Yang, H.; Pan, J.; Liu, J.; Dotse, C.; Luan, Y.; Gao, R.; Lin, C.; Zhang, J.; Kilcrease, J. P.; Wen, X.; Zou, S.; Fang, J. High-Indexed Pt 3 Ni Alloy Tetrahexahedral Nanoframes Evolved through Preferential CO Etching. Nano Lett. 2017, 17, 2204-2210.

(255) Li, J.; Jilani, S. Z.; Lin, H.; Liu, X.; Wei, K.; Jia, Y.; Zhang, P.; Chi, M.; Tong, Y. J.; Xi, Z.; Sun, S. Ternary CoPtAu Nanoparticles as a General Catalyst for Highly Efficient Electro-oxidation of Liquid Fuels. Angew. Chemie Int. Ed. 2019, 58, 11527-11533.

(256) Zhang, S.; Guo, S.; Zhu, H.; Su, D.; Sun, S. Structure-Induced Enhancement in Electrooxidation of Trimetallic FePtAu Nanoparticles. J. Am. Chem. Soc. 2012, 134, $5060-5063$.

(257) Li, J.; Sun, S. Intermetallic Nanoparticles: Synthetic Control and Their Enhanced Electrocatalysis. Acc. Chem. Res. 2019, 52, 2015-2025.

(258) Li, J.; Jilani, S. Z.; Lin, H.; Liu, X.; Wei, K.; Jia, Y.; Zhang, P.; Chi, M.; Tong, Y. J.; Xi, Z.; Sun, S. Ternary CoPtAu Nanoparticles as a General Catalyst for Highly Efficient Electro-oxidation of Liquid Fuels. Angew. Chemie Int. Ed. 2019, 58, 11527-11533.

(259) Ferrin, P.; Mavrikakis, M. Structure Sensitivity of Methanol Electrooxidation on Transition Metals. J. Am. Chem. Soc. 2009, 131, 14381-14389.

(260) Han, S.-B.; Song, Y.-J.; Lee, J.-M.; Kim, J.-Y.; Park, K.-W. Platinum Nanocube Catalysts for Methanol and Ethanol Electrooxidation. Electrochem. commun. 2008, 10, $1044-1047$.

(261) Morallón, E.; Rodes, A.; Vázquez, J. L.; Pérez, J. M. Voltammetric and In-Situ FTIR Spectroscopic Study of the Oxidation of Methanol on Pt(Hkl) in Alkaline Media. $J$. Electroanal. Chem. 1995, 391, 149-157.

(262) Solla-Gullón, J.; Vidal-Iglesias, F. J.; López-Cudero, A.; Garnier, E.; Feliu, J. M.; Aldaz, A. Shape-Dependent Electrocatalysis: Methanol and Formic Acid Electrooxidation on Preferentially Oriented Pt Nanoparticles. Phys. Chem. Chem. Phys. 2008, 10, 3689.

(263) Klein, J.; Brimaud, S.; Engstfeld, A. K.; Behm, R. J. Atomic Scale Insights on the Electronic and Geometric Effects in the Electro-Oxidation of CO on PtxRu1-x/Ru(0001) 
Surface Alloys. Electrochim. Acta 2019, 306, 516-528.

(264) Zhang, N.; Bu, L.; Guo, S.; Guo, J.; Huang, X. Screw Thread-Like Platinum-Copper Nanowires Bounded with High-Index Facets for Efficient Electrocatalysis. Nano Lett. 2016, 16, 5037-5043.

(265) Bu, L.; Guo, S.; Zhang, X.; Shen, X.; Su, D.; Lu, G.; Zhu, X.; Yao, J.; Guo, J.; Huang, $X$. Surface Engineering of Hierarchical Platinum-Cobalt Nanowires for Efficient Electrocatalysis. Nat. Commun. 2016, 7, 11850.

(266) Sun, X.; Li, D.; Guo, S.; Zhu, W.; Sun, S. Controlling Core/Shell Au/FePt Nanoparticle Electrocatalysis via Changing the Core Size and Shell Thickness. Nanoscale 2016, 8, 2626-2631.

(267) Sun, X.; Li, D.; Ding, Y.; Zhu, W.; Guo, S.; Wang, Z. L.; Sun, S. Core/Shell Au/CuPt Nanoparticles and Their Dual Electrocatalysis for Both Reduction and Oxidation Reactions. J. Am. Chem. Soc. 2014, 136, 5745-5749.

(268) Li, M.; Zhao, Z.; Xia, Z.; Yang, Y.; Luo, M.; Huang, Y.; Sun, Y.; Chao, Y.; Yang, W.; Yang, W.; Yu, Y.; Lu, G.; Guo, S. Lavender-Like Ga-Doped Pt3Co Nanowires for Highly Stable and Active Electrocatalysis. ACS Catal. 2020, 10, 3018-3026.

(269) Huang, L.; Zhang, X.; Wang, Q.; Han, Y.; Fang, Y.; Dong, S. Shape-Control of Pt-Ru Nanocrystals: Tuning Surface Structure for Enhanced Electrocatalytic Methanol Oxidation. J. Am. Chem. Soc. 2018, 140, 1142-1147.

(270) Tan, C.; Sun, Y.; Zheng, J.; Wang, D.; Li, Z.; Zeng, H.; Guo, J.; Jing, L.; Jiang, L. A Self-Supporting Bimetallic Au@Pt Core-Shell Nanoparticle Electrocatalyst for the Synergistic Enhancement of Methanol Oxidation. Sci. Rep. 2017, 7, 6347.

(271) Lu, S.; Eid, K.; Ge, D.; Guo, J.; Wang, L.; Wang, H.; Gu, H. One-Pot Synthesis of PtRu Nanodendrites as Efficient Catalysts for Methanol Oxidation Reaction. Nanoscale 2017, 9, 1033-1039.

(272) Yin, A.-X.; Min, X.-Q.; Zhang, Y.-W.; Yan, C.-H. Shape-Selective Synthesis and FacetDependent Enhanced Electrocatalytic Activity and Durability of Monodisperse Sub-10 Nm Pt-Pd Tetrahedrons and Cubes. J. Am. Chem. Soc. 2011, 133, 3816-3819.

(273) Guo, S.; Zhang, S.; Sun, X.; Sun, S. Synthesis of Ultrathin FePtPd Nanowires and Their Use as Catalysts for Methanol Oxidation Reaction. J. Am. Chem. Soc. 2011, 133, 1535415357.

(274) Kelly, C. H. W.; Benedetti, T. M.; Alinezhad, A.; Schuhmann, W.; Gooding, J. J.; Tilley, R. D. Understanding the Effect of $\mathrm{Au}$ in $\mathrm{Au}-\mathrm{Pd}$ Bimetallic Nanocrystals on the Electrocatalysis of the Methanol Oxidation Reaction. J. Phys. Chem. C 2018, 122, 
$21718-21723$.

(275) Li, J.; Luo, Z.; He, F.; Zuo, Y.; Zhang, C.; Liu, J.; Yu, X.; Du, R.; Zhang, T.; InfanteCarrió, M. F.; Tang, P.; Arbiol, J.; Llorca, J.; Cabot, A. Colloidal Ni-Co-Sn Nanoparticles as Efficient Electrocatalysts for the Methanol Oxidation Reaction. $J$. Mater. Chem. A 2018, 6, 22915-22924.

(276) Du, H.; Luo, S.; Wang, K.; Tang, M.; Sriphathoorat, R.; Jin, Y.; Shen, P. K. HighQuality and Deeply Excavated Pt3Co Nanocubes as Efficient Catalysts for Liquid Fuel Electrooxidation. Chem. Mater. 2017, 29, 9613-9617.

(277) Chen, Q.; Yang, Y.; Cao, Z.; Kuang, Q.; Du, G.; Jiang, Y.; Xie, Z.; Zheng, L. Excavated Cubic Platinum-Tin Alloy Nanocrystals Constructed from Ultrathin Nanosheets with Enhanced Electrocatalytic Activity. Angew. Chemie Int. Ed. 2016, 55, 9021-9025.

(278) Lv, H.; Xu, D.; Sun, L.; Henzie, J.; Suib, S. L.; Yamauchi, Y.; Liu, B. Ternary Palladium-Boron-Phosphorus Alloy Mesoporous Nanospheres for Highly Efficient Electrocatalysis. ACS Nano 2019, 13, 12052-12061.

(279) Lu, W.; Xia, X.; Wei, X.; Li, M.; Zeng, M.; Guo, J.; Cheng, S. Nanoengineering 2D Dendritic PdAgPt Nanoalloys with Edge-Enriched Active Sites for Enhanced Alcohol Electroxidation and Electrocatalytic Hydrogen Evolution. ACS Appl. Mater. Interfaces 2020, 12, 21569-21578.

(280) Huang, J.; Liu, Y.; Xu, M.; Wan, C.; Liu, H.; Li, M.; Huang, Z.; Duan, X.; Pan, X.; Huang, Y. PtCuNi Tetrahedra Catalysts with Tailored Surfaces for Efficient Alcohol Oxidation. Nano Lett. 2019, 19, 5431-5436.

(281) Ji, Q.; Zhou, Y.; Xiang, C.; Zhang, G.; Li, J.; Liu, H.; Qu, J. Manipulation of Neighboring Palladium and Mercury Atoms for Efficient $* \mathrm{OH}$ Transformation in Anodic Alcohol Oxidation and Cathodic Oxygen Reduction Reactions. ACS Appl. Mater. Interfaces 2020, 12, 12677-12685.

(282) Huang, X.; Zhao, Z.; Fan, J.; Tan, Y.; Zheng, N. Amine-Assisted Synthesis of Concave Polyhedral Platinum Nanocrystals Having $\{411\}$ High-Index Facets. J. Am. Chem. Soc. 2011, 133, 4718-4721.

(283) Yuan, Q.; Zhou, Z.; Zhuang, J.; Wang, X. Seed Displacement, Epitaxial Synthesis of $\mathrm{Rh} / \mathrm{Pt}$ Bimetallic Ultrathin Nanowires for Highly Selective Oxidizing Ethanol to CO 2. Chem. Mater. 2010, 22, 2395-2402.

(284) Ye, S. J.; Kim, D. Y.; Kang, S. W.; Choi, K. W.; Han, S. W.; Park, O. O. Synthesis of Chestnut-Bur-like Palladium Nanostructures and Their Enhanced Electrocatalytic Activities for Ethanol Oxidation. Nanoscale 2014, 6, 4182-4187. 
(285) Jin, L.; Xu, H.; Chen, C.; Shang, H.; Wang, Y.; Du, Y. Superior Ethanol Oxidation Electrocatalysis Enabled by Ternary Pd-Rh-Te Nanotubes. Inorg. Chem. 2019, 58, 12377-12384.

(286) Rizo, R.; Arán-Ais, R. M.; Padgett, E.; Muller, D. A.; Lázaro, M. J.; Solla-Gullón, J.; Feliu, J. M.; Pastor, E.; Abruña, H. D. Pt-Rich Core /Sn-Rich Subsurface /Pt Skin Nanocubes As Highly Active and Stable Electrocatalysts for the Ethanol Oxidation Reaction. J. Am. Chem. Soc. 2018, 140, 3791-3797.

(287) Erini, N.; Loukrakpam, R.; Petkov, V.; Baranova, E. A.; Yang, R.; Teschner, D.; Huang, Y.; Brankovic, S. R.; Strasser, P. Ethanol Electro-Oxidation on Ternary PlatinumRhodium-Tin Nanocatalysts: Insights in the Atomic 3D Structure of the Active Catalytic Phase. ACS Catal. 2014, 4, 1859-1867.

(288) Luo, Z.; Lu, J.; Flox, C.; Nafria, R.; Genç, A.; Arbiol, J.; Llorca, J.; Ibáñez, M.; Morante, J. R.; Cabot, A. Pd2Sn [010] Nanorods as a Highly Active and Stable Ethanol Oxidation Catalyst. J. Mater. Chem. A 2016, 4, 16706-16713.

(289) Zhao, X.; Dai, L.; Qin, Q.; Pei, F.; Hu, C.; Zheng, N. Self-Supported 3D PdCu Alloy Nanosheets as a Bifunctional Catalyst for Electrochemical Reforming of Ethanol. Small 2017, 13, 1602970.

(290) Zhao, M.; Lyu, Z.; Xie, M.; Hood, Z. D.; Cao, Z.; Chi, M.; Xia, Y. Pd-Ru Alloy Nanocages with a Face-Centered Cubic Structure and Their Enhanced Activity toward the Oxidation of Ethylene Glycol and Glycerol. Small Methods 2020, 4, 1900843.

(291) Kim, D.; Lee, Y. W.; Lee, S. B.; Han, S. W. Convex Polyhedral Au@Pd Core-Shell Nanocrystals with High-Index Facets. Angew. Chemie Int. Ed. 2012, 51, 159-163.

(292) Rao, L.; Jiang, Y.-X.; Zhang, B.-W.; Cai, Y.-R.; Sun, S.-G. High Activity of Cubic PtRh Alloys Supported on Graphene towards Ethanol Electrooxidation. Phys. Chem. Chem. Phys. 2014, 16, 13662.

(293) Erini, N.; Beermann, V.; Gocyla, M.; Gliech, M.; Heggen, M.; Dunin-Borkowski, R. E.; Strasser, P. The Effect of Surface Site Ensembles on the Activity and Selectivity of Ethanol Electrooxidation by Octahedral PtNiRh Nanoparticles. Angew. Chemie Int. Ed. 2017, 56, 6533-6538.

(294) Almeida, T. S.; Yu, Y.; de Andrade, A. R.; Abruña, H. D. Employing Iron and Nickel to Enhance Ethanol Oxidation of Pd-Based Anodes in Alkaline Medium. Electrochim. Acta 2019, 295, 751-758.

(295) Zhang, J.; Hou, C.; Huang, H.; Zhang, L.; Jiang, Z.; Chen, G.; Jia, Y.; Kuang, Q.; Xie, Z; Zheng, L. Surfactant-Concentration-Dependent Shape Evolution of Au-Pd Alloy 
Nanocrystals from Rhombic Dodecahedron to Trisoctahedron and Hexoctahedron. Small 2013, 9, 538-544.

(296) Lin, H.; Muzzio, M.; Wei, K.; Zhang, P.; Li, J.; Li, N.; Yin, Z.; Su, D.; Sun, S. PdAu Alloy Nanoparticles for Ethanol Oxidation in Alkaline Conditions: Enhanced Activity and C1 Pathway Selectivity. ACS Appl. Energy Mater. 2019, 2, 8701-8706.

(297) Lee, Y. W.; Kim, M.; Kang, S. W.; Han, S. W. Polyhedral Bimetallic Alloy Nanocrystals Exclusively Bound by $\{110\}$ Facets: Au-Pd Rhombic Dodecahedra. Angew. Chemie Int. Ed. 2011, 50, 3466-3470.

(298) Dutta, S.; Ray, C.; Sasmal, A. K.; Negishi, Y.; Pal, T. Fabrication of Dog-Bone Shaped Au NR Core-Pt/Pd Shell Trimetallic Nanoparticle-Decorated Reduced Graphene Oxide Nanosheets for Excellent Electrocatalysis. J. Mater. Chem. A 2016, 4, 3765-3776.

(299) Otor, H. O.; Steiner, J. B.; García-Sancho, C.; Alba-Rubio, A. C. Encapsulation Methods for Control of Catalyst Deactivation: A Review. ACS Catal. 2020, 10, 7630-7656.

(300) Yang, K.; Kas, R.; Smith, W. A. In Situ Infrared Spectroscopy Reveals Persistent Alkalinity near Electrode Surfaces during CO2 Electroreduction. J. Am. Chem. Soc. 2019, 141, 15891-15900.

(301) Peterson, A. A.; Nørskov, J. K. Activity Descriptors for CO2 Electroreduction to Methane on Transition-Metal Catalysts. J. Phys. Chem. Lett. 2012, 3, 251-258.

(302) Hansen, H. A.; Varley, J. B.; Peterson, A. A.; Nørskov, J. K. Understanding Trends in the Electrocatalytic Activity of Metals and Enzymes for CO2 Reduction to CO. J. Phys. Chem. Lett. 2013, 4, 388-392.

(303) Benson, E. E.; Zhang, H.; Schuman, S. A.; Nanayakkara, S. U.; Bronstein, N. D.; Ferrere, S.; Blackburn, J. L.; Miller, E. M. Balancing the Hydrogen Evolution Reaction, Surface Energetics, and Stability of Metallic MoS2 Nanosheets via Covalent Functionalization. J. Am. Chem. Soc. 2018, 140, 441-450.

(304) Tappan, B. A.; Chen, K.; Lu, H.; Sharada, S. M.; Brutchey, R. L. Synthesis and Electrocatalytic HER Studies of Carbene-Ligated Cu3-XP Nanocrystals. ACS Appl. Mater. Interfaces 2020, 12, 16394-16401.

(305) Creus, J.; Drouet, S.; Suriñach, S.; Lecante, P.; Collière, V.; Poteau, R.; Philippot, K.; García-Antón, J.; Sala, X. Ligand-Capped Ru Nanoparticles as Efficient Electrocatalyst for the Hydrogen Evolution Reaction. ACS Catal. 2018, 8, 11094-11102.

(306) Zhou, Z.-Y.; Kang, X.; Song, Y.; Chen, S. Ligand-Mediated Electrocatalytic Activity of Pt Nanoparticles for Oxygen Reduction Reactions. J. Phys. Chem. C 2012, 116, 1059210598. 
(307) Deming, C. P.; Zhao, A.; Song, Y.; Liu, K.; Khan, M. M.; Yates, V. M.; Chen, S. Alkyne-Protected AuPd Alloy Nanoparticles for Electrocatalytic Reduction of Oxygen. ChemElectroChem 2015, 2, 1719-1727.

(308) Hu, P.; Song, Y.; Chen, L.; Chen, S. Electrocatalytic Activity of Alkyne-Functionalized $\mathrm{AgAu}$ Alloy Nanoparticles for Oxygen Reduction in Alkaline Media. Nanoscale 2015, 7, 9627-9636.

(309) Zhou, Z.-Y.; Kang, X.; Song, Y.; Chen, S. Enhancement of the Electrocatalytic Activity of Pt Nanoparticles in Oxygen Reduction by Chlorophenyl Functionalization. Chem. Commun. 2012, 48, 3391.

(310) Alba-Molina, D.; Puente Santiago, A. R.; Giner-Casares, J. J.; Rodríguez-Castellón, E.; Martín-Romero, M. T.; Camacho, L.; Luque, R.; Cano, M. Tailoring the ORR and HER Electrocatalytic Performances of Gold Nanoparticles through Metal-Ligand Interfaces. J. Mater. Chem. A 2019, 7, 20425-20434.

(311) Cheng, N.; Li, H.; Li, G.; Lv, H.; Mu, S.; Sun, X.; Pan, M. Highly Active Pt@Au Nanoparticles Encapsulated in Perfluorosulfonic Acid for the Reduction of Oxygen. Chem. Commun. 2011, 47, 12792.

(312) Mirkhalaf, F.; Schiffrin, D. J. Electrocatalytic Oxygen Reduction on Functionalized Gold Nanoparticles Incorporated in a Hydrophobic Environment. Langmuir 2010, 26, 14995-15001.

(313) Miyabayashi, K.; Nishihara, H.; Miyake, M. Platinum Nanoparticles Modified with Alkylamine Derivatives as an Active and Stable Catalyst for Oxygen Reduction Reaction. Langmuir 2014, 30, 2936-2942.

(314) Ahn, S.; Klyukin, K.; Wakeham, R. J.; Rudd, J. A.; Lewis, A. R.; Alexander, S.; Carla, F.; Alexandrov, V.; Andreoli, E. Poly-Amide Modified Copper Foam Electrodes for Enhanced Electrochemical Reduction of Carbon Dioxide. ACS Catal. 2018, 8, 41324142 .

(315) Cao, Z.; Derrick, J. S.; Xu, J.; Gao, R.; Gong, M.; Nichols, E. M.; Smith, P. T.; Liu, X.; Wen, X.; Copéret, C.; Chang, C. J. Chelating N-Heterocyclic Carbene Ligands Enable Tuning of Electrocatalytic CO 2 Reduction to Formate and Carbon Monoxide: Surface Organometallic Chemistry. Angew. Chemie Int. Ed. 2018, 57, 4981-4985.

(316) Wang, Z.; Wu, L.; Sun, K.; Chen, T.; Jiang, Z.; Cheng, T.; Goddard, W. A. Surface Ligand Promotion of Carbon Dioxide Reduction through Stabilizing Chemisorbed Reactive Intermediates. J. Phys. Chem. Lett. 2018, 9, 3057-3061.

(317) Li, F.; Tang, Q. Understanding the Role of Functional Groups of Thiolate Ligands in 
Electrochemical CO 2 Reduction over Au(111) from First-Principles. J. Mater. Chem. A 2019, 7, 19872-19880.

(318) Kim, C.; Jeon, H. S.; Eom, T.; Jee, M. S.; Kim, H.; Friend, C. M.; Min, B. K.; Hwang, Y. J. Achieving Selective and Efficient Electrocatalytic Activity for CO 2 Reduction Using Immobilized Silver Nanoparticles. J. Am. Chem. Soc. 2015, 137, 13844-13850.

(319) Wang, Z.; Sun, K.; Liang, C.; Wu, L.; Niu, Z.; Gao, J. Synergistic Chemisorbing and Electronic Effects for Efficient CO 2 Reduction Using Cysteamine-Functionalized Gold Nanoparticles. ACS Appl. Energy Mater. 2019, 2, 192-195.

(320) Fang, Y.; Flake, J. C. Electrochemical Reduction of $\mathrm{CO} 2$ at Functionalized $\mathrm{Au}$ Electrodes. J. Am. Chem. Soc. 2017, 139, 3399-3405.

(321) Pankhurst, J. R.; Guntern, Y. T.; Mensi, M.; Buonsanti, R. Molecular Tunability of Surface-Functionalized Metal Nanocrystals for Selective Electrochemical CO2 Reduction. Chem. Sci. 2019, 10, 10356-10365.

(322) Wakerley, D.; Lamaison, S.; Ozanam, F.; Menguy, N.; Mercier, D.; Marcus, P.; Fontecave, M.; Mougel, V. Bio-Inspired Hydrophobicity Promotes CO2 Reduction on a Cu Surface. Nat. Mater. 2019, 18, 1222-1227.

(323) Buckley, A. K.; Lee, M.; Cheng, T.; Kazantsev, R. V.; Larson, D. M.; Goddard III, W. A.; Toste, F. D.; Toma, F. M. Electrocatalysis at Organic-Metal Interfaces: Identification of Structure-Reactivity Relationships for CO2 Reduction at Modified $\mathrm{Cu}$ Surfaces. J. Am. Chem. Soc. 2019, 141, 7355-7364.

(324) Liang, Y.; Li, Y.; Wang, H.; Dai, H. Strongly Coupled Inorganic/Nanocarbon Hybrid Materials for Advanced Electrocatalysis. J. Am. Chem. Soc. 2013, 135, 2013-2036.

(325) Dresp, S.; Luo, F.; Schmack, R.; Kühl, S.; Gliech, M.; Strasser, P. An Efficient Bifunctional Two-Component Catalyst for Oxygen Reduction and Oxygen Evolution in Reversible Fuel Cells, Electrolyzers and Rechargeable Air Electrodes. Energy Environ. Sci. 2016, 9, 2020-2024.

(326) Rogers, C.; Perkins, W. S.; Veber, G.; Williams, T. E.; Cloke, R. R.; Fischer, F. R. Synergistic Enhancement of Electrocatalytic CO2 Reduction with Gold Nanoparticles Embedded in Functional Graphene Nanoribbon Composite Electrodes. J. Am. Chem. Soc. 2017, 139, 4052-4061.

(327) Li, F.; MacFarlane, D. R.; Zhang, J. Recent Advances in the Nanoengineering of Electrocatalysts for CO2 Reduction. Nanoscale 2018, 10, 6235-6260.

(328) Guo, S.; Sun, S. FePt Nanoparticles Assembled on Graphene as Enhanced Catalyst for Oxygen Reduction Reaction. J. Am. Chem. Soc. 2012, 134, 2492-2495. 
(329) Liang, Y.; Li, Y.; Wang, H.; Zhou, J.; Wang, J.; Regier, T.; Dai, H. Co3O4 Nanocrystals on Graphene as a Synergistic Catalyst for Oxygen Reduction Reaction. Nat. Mater. 2011, 10, 780-786.

(330) Liang, Y.; Wang, H.; Zhou, J.; Li, Y.; Wang, J.; Regier, T.; Dai, H. Covalent Hybrid of Spinel Manganese-Cobalt Oxide and Graphene as Advanced Oxygen Reduction Electrocatalysts. J. Am. Chem. Soc. 2012, 134, 3517-3523.

(331) Huang, J.; Guo, X.; Yue, G.; Hu, Q.; Wang, L. Boosting CH3OH Production in Electrocatalytic CO2 Reduction over Partially Oxidized $5 \mathrm{Nm}$ Cobalt Nanoparticles Dispersed on Single-Layer Nitrogen-Doped Graphene. ACS Appl. Mater. Interfaces 2018, 10, 44403-44414.

(332) Zhang, L.; Mao, F.; Zheng, L. R.; Wang, H. F.; Yang, X. H.; Yang, H. G. Tuning Metal Catalyst with Metal-C3N4 Interaction for Efficient CO2 Electroreduction. ACS Catal. 2018, 8, 11035-11041.

(333) Lee, J. H.; Kattel, S.; Xie, Z.; Tackett, B. M.; Wang, J.; Liu, C.-J.; Chen, J. G. Understanding the Role of Functional Groups in Polymeric Binder for Electrochemical Carbon Dioxide Reduction on Gold Nanoparticles. Adv. Funct. Mater. 2018, 28, 1804762.

(334) Liu, H.; Xiang, K.; Liu, Y.; Zhu, F.; Zou, M.; Yan, X.; Chai, L. Polydopamine Functionalized $\mathrm{Cu}$ Nanowires for Enhanced CO2 Electroreduction Towards Methane. ChemElectroChem 2018, 5, 3991-3999.

(335) Zhang, L.; Wei, Z.; Thanneeru, S.; Meng, M.; Kruzyk, M.; Ung, G.; Liu, B.; He, J. A Polymer Solution To Prevent Nanoclustering and Improve the Selectivity of Metal Nanoparticles for Electrocatalytic CO2 Reduction. Angew. Chemie Int. Ed. 2019, 58, 15834-15840.

(336) Thevenon, A.; Rosas-Hernández, A.; Peters, J. C.; Agapie, T. In-Situ Nanostructuring and Stabilization of Polycrystalline Copper by an Organic Salt Additive Promotes Electrocatalytic CO2 Reduction to Ethylene. Angew. Chemie Int. Ed. 2019, 58, 1695216958.

(337) Han, Z.; Kortlever, R.; Chen, H.-Y.; Peters, J. C.; Agapie, T. CO2 Reduction Selective for $\mathrm{C} \geq 2$ Products on Polycrystalline Copper with N-Substituted Pyridinium Additives. ACS Cent. Sci. 2017, 3, 853-859.

(338) Li, F.; Thevenon, A.; Rosas-Hernández, A.; Wang, Z.; Li, Y.; Gabardo, C. M.; Ozden, A.; Dinh, C. T.; Li, J.; Wang, Y.; Edwards, J. P.; Xu, Y.; McCallum, C.; Tao, L.; Liang, Z.-Q.; Luo, M.; Wang, X.; Li, H.; O’Brien, C. P.; Tan, C.-S.; Nam, D.-H.; Quintero- 
Bermudez, R.; Zhuang, T.-T.; Li, Y. C.; Han, Z.; Britt, R. D.; Sinton, D.; Agapie, T.; Peters, J. C.; Sargent, E. H. Molecular Tuning of CO2-to-Ethylene Conversion. Nature 2020, 577, 509-513.

(339) Xu, G.-R.; Bai, J.; Yao, L.; Xue, Q.; Jiang, J.-X.; Zeng, J.-H.; Chen, Y.; Lee, J.-M. Polyallylamine-Functionalized Platinum Tripods: Enhancement of Hydrogen Evolution Reaction by Proton Carriers. ACS Catal. 2017, 7, 452-458.

(340) Yang, Q.; Xu, Q.; Jiang, H.-L. Metal-Organic Frameworks Meet Metal Nanoparticles: Synergistic Effect for Enhanced Catalysis. Chem. Soc. Rev. 2017, 46, 4774-4808.

(341) Kung, C.-W.; Audu, C. O.; Peters, A. W.; Noh, H.; Farha, O. K.; Hupp, J. T. Copper Nanoparticles Installed in Metal-Organic Framework Thin Films Are Electrocatalytically Competent for CO2 Reduction. ACS Energy Lett. 2017, 2, 23942401.

(342) Guntern, Y. T.; Pankhurst, J. R.; Vávra, J.; Mensi, M.; Mantella, V.; Schouwink, P.; Buonsanti, R. Nanocrystal/Metal-Organic Framework Hybrids as Electrocatalytic Platforms for CO2 Conversion. Angew. Chemie Int. Ed. 2019, 58, 12632-12639.

(343) Heidary, N.; Morency, M.; Chartrand, D.; Ly, K. H.; Iftimie, R.; Kornienko, N. Electrochemically Triggered Dynamics within a Hybrid Metal-Organic Electrocatalyst. J. Am. Chem. Soc. 2020, 142, 12382-12393.

(344) Spöri, C.; Kwan, J. T. H.; Bonakdarpour, A.; Wilkinson, D. P.; Strasser, P. The Stability Challenges of Oxygen Evolving Catalysts: Towards a Common Fundamental Understanding and Mitigation of Catalyst Degradation. Angew. Chemie Int. Ed. 2017, 56, 5994-6021.

(345) Li, Y.; Hart, J. L.; Taheri, M. L.; Snyder, J. D. Morphological Instability in Topologically Complex, Three-Dimensional Electrocatalytic Nanostructures. ACS Catal. 2017, 7, 7995-8005.

(346) Chattot, R.; Le Bacq, O.; Beermann, V.; Kühl, S.; Herranz, J.; Henning, S.; Kühn, L.; Asset, T.; Guétaz, L.; Renou, G.; Drnec, J.; Bordet, P.; Pasturel, A.; Eychmüller, A.; Schmidt, T. J.; Strasser, P.; Dubau, L.; Maillard, F. Surface Distortion as a Unifying Concept and Descriptor in Oxygen Reduction Reaction Electrocatalysis. Nat. Mater. 2018, 17, 827-833.

(347) Baldizzone, C.; Gan, L.; Hodnik, N.; Keeley, G. P.; Kostka, A.; Heggen, M.; Strasser, P.; Mayrhofer, K. J. J. Stability of Dealloyed Porous Pt/Ni Nanoparticles. ACS Catal. 2015, 5, 5000-5007.

(348) Rasouli, S.; Myers, D.; Kariuki, N.; Higashida, K.; Nakashima, N.; Ferreira, P. 
Electrochemical Degradation of Pt-Ni Nanocatalysts: An Identical Location AberrationCorrected Scanning Transmission Electron Microscopy Study. Nano Lett. 2019, 19, 4653.

(349) Hodnik, N.; Zorko, M.; Bele, M.; Hočevar, S.; Gaberšček, M. Identical Location Scanning Electron Microscopy: A Case Study of Electrochemical Degradation of PtNi Nanoparticles Using a New Nondestructive Method. J. Phys. Chem. C 2012, 116, $21326-21333$.

(350) Osowiecki, W. T.; Nussbaum, J. J.; Kamat, G. A.; Katsoukis, G.; Ledendecker, M.; Frei, H.; Bell, A. T.; Alivisatos, A. P. Factors and Dynamics of $\mathrm{Cu}$ Nanocrystal Reconstruction under CO2 Reduction. ACS Appl. Energy Mater. 2019, 2, 7744-7749.

(351) Vavra, J.; Shen, T. T.-H.; Stoian, D.; Tileli, V.; Buonsanti, R. Real-time Monitoring Reveals Dissolution/Redeposition Mechanism in Copper Nanocatalysts during the Initial Stages of the $\mathrm{CO} 2$ Reduction Reaction. Angew. Chemie Int. Ed. 2020, anie.202011137.

(352) Li, Y.; Kim, D.; Louisia, S.; Xie, C.; Kong, Q.; Yu, S.; Lin, T.; Aloni, S.; Fakra, S. C.; Yang, P. Electrochemically Scrambled Nanocrystals Are Catalytically Active for CO2 -to-Multicarbons. Proc. Natl. Acad. Sci. 2020, 117, 9194-9201.

(353) Jung, H.; Lee, S. Y.; Lee, C. W.; Cho, M. K.; Won, D. H.; Kim, C.; Oh, H.-S.; Min, B. K.; Hwang, Y. J. Electrochemical Fragmentation of $\mathrm{Cu} 2 \mathrm{O}$ Nanoparticles Enhancing Selective C-C Coupling from CO2 Reduction Reaction. J. Am. Chem. Soc. 2019, 141, 4624-4633.

(354) Varandili, S. B.; Huang, J.; Oveisi, E.; De Gregorio, G. L.; Mensi, M.; Strach, M.; Vavra, J.; Gadiyar, C.; Bhowmik, A.; Buonsanti, R. Synthesis of $\mathrm{Cu} / \mathrm{CeO} 2-\mathrm{x}$ Nanocrystalline Heterodimers with Interfacial Active Sites To Promote CO2 Electroreduction. ACS Catal. 2019, 9, 5035-5046.

(355) Zheng, X.; Ji, Y.; Tang, J.; Wang, J.; Liu, B.; Steinrück, H.-G.; Lim, K.; Li, Y.; Toney, M. F.; Chan, K.; Cui, Y. Theory-Guided $\mathrm{Sn} / \mathrm{Cu}$ Alloying for Efficient CO2 Electroreduction at Low Overpotentials. Nat. Catal. 2019, 2, 55-61.

(356) Weng, Z.; Wu, Y.; Wang, M.; Jiang, J.; Yang, K.; Huo, S.; Wang, X.-F.; Ma, Q.; Brudvig, G. W.; Batista, V. S.; Liang, Y.; Feng, Z.; Wang, H. Active Sites of CopperComplex Catalytic Materials for Electrochemical Carbon Dioxide Reduction. Nat. Commun. 2018, 9, 415.

(357) Firet, N. J.; Blommaert, M. A.; Burdyny, T.; Venugopal, A.; Bohra, D.; Longo, A.; Smith, W. A. Operando EXAFS Study Reveals Presence of Oxygen in Oxide-Derived 
Silver Catalysts for Electrochemical CO2 Reduction. J. Mater. Chem. A 2019, 7, 25972607.

(358) Kornienko, N.; Resasco, J.; Becknell, N.; Jiang, C.-M.; Liu, Y.-S.; Nie, K.; Sun, X.; Guo, J.; Leone, S. R.; Yang, P. Operando Spectroscopic Analysis of an Amorphous Cobalt Sulfide Hydrogen Evolution Electrocatalyst. J. Am. Chem. Soc. 2015, 137, 74487455.

(359) Ampurdanés, J.; Chourashiya, M.; Urakawa, A. Cobalt Oxide-Based Materials as NonPGM Catalyst for HER in PEM Electrolysis and in Situ XAS Characterization of Its Functional State. Catal. Today 2019, 336, 161-168.

(360) Friebel, D.; Louie, M. W.; Bajdich, M.; Sanwald, K. E.; Cai, Y.; Wise, A. M.; Cheng, M.-J.; Sokaras, D.; Weng, T.-C.; Alonso-Mori, R.; Davis, R. C.; Bargar, J. R.; Nørskov, J. K.; Nilsson, A.; Bell, A. T. Identification of Highly Active Fe Sites in (Ni,Fe)OOH for Electrocatalytic Water Splitting. J. Am. Chem. Soc. 2015, 137, 1305-1313.

(361) Gibbons, B. M.; Wette, M.; Stevens, M. B.; Davis, R. C.; Siahrostami, S.; Kreider, M.; Mehta, A.; Higgins, D. C.; Clemens, B. M.; Jaramillo, T. F. In Situ X-Ray Absorption Spectroscopy Disentangles the Roles of Copper and Silver in a Bimetallic Catalyst for the Oxygen Reduction Reaction. Chem. Mater. 2020, 32, 1819-1827.

(362) Gorlin, Y.; Lassalle-Kaiser, B.; Benck, J. D.; Gul, S.; Webb, S. M.; Yachandra, V. K.; Yano, J.; Jaramillo, T. F. In Situ X-Ray Absorption Spectroscopy Investigation of a Bifunctional Manganese Oxide Catalyst with High Activity for Electrochemical Water Oxidation and Oxygen Reduction. J. Am. Chem. Soc. 2013, 135, 8525-8534.

(363) Wang, M.; Árnadóttir, L.; Xu, Z. J.; Feng, Z. In Situ X-Ray Absorption Spectroscopy Studies of Nanoscale Electrocatalysts. Nano-Micro Lett. 2019, 11, 47.

(364) Li, X.; Wang, S.; Li, L.; Sun, Y.; Xie, Y. Progress and Perspective for In Situ Studies of CO2 Reduction. J. Am. Chem. Soc. 2020, 142, 9567-9581.

(365) Zhu, Y.; Wang, J.; Chu, H.; Chu, Y.-C.; Chen, H. M. In Situ / Operando Studies for Designing Next-Generation Electrocatalysts. ACS Energy Lett. 2020, 5, 1281-1291.

(366) Handoko, A. D.; Wei, F.; Jenndy; Yeo, B. S.; Seh, Z. W. Understanding Heterogeneous Electrocatalytic Carbon Dioxide Reduction through Operando Techniques. Nat. Catal. 2018, 1, 922-934.

(367) Lassalle-Kaiser, B.; Gul, S.; Kern, J.; Yachandra, V. K.; Yano, J. In Situ/Operando Studies of Electrocatalysts Using Hard X-Ray Spectroscopy. J. Electron Spectros. Relat. Phenomena 2017, 221, 18-27.

(368) Choi, Y.-W.; Mistry, H.; Roldan Cuenya, B. New Insights into Working Nanostructured 
Electrocatalysts through Operando Spectroscopy and Microscopy. Curr. Opin. Electrochem. 2017, 1, 95-103.

(369) Jeon, H. S.; Timoshenko, J.; Scholten, F.; Sinev, I.; Herzog, A.; Haase, F. T.; Roldan Cuenya, B. Operando Insight into the Correlation between the Structure and Composition of CuZn Nanoparticles and Their Selectivity for the Electrochemical CO2 Reduction. J. Am. Chem. Soc. 2019, 141, 19879-19887.

(370) Farmand, M.; Landers, A. T.; Lin, J. C.; Feaster, J. T.; Beeman, J. W.; Ye, Y.; Clark, E. L.; Higgins, D.; Yano, J.; Davis, R. C.; Mehta, A.; Jaramillo, T. F.; Hahn, C.; Drisdell, W. S. Electrochemical Flow Cell Enabling Operando Probing of Electrocatalyst Surfaces by X-Ray Spectroscopy and Diffraction. Phys. Chem. Chem. Phys. 2019, 21, $5402-5408$.

(371) Dutta, A.; Kuzume, A.; Rahaman, M.; Vesztergom, S.; Broekmann, P. Monitoring the Chemical State of Catalysts for CO2 Electroreduction: An In Operando Study. ACS Catal. 2015, 5, 7498-7502.

(372) Ren, D.; Gao, J.; Pan, L.; Wang, Z.; Luo, J.; Zakeeruddin, S. M.; Hagfeldt, A.; Grätzel, M. Atomic Layer Deposition of $\mathrm{ZnO}$ on $\mathrm{CuO}$ Enables Selective and Efficient Electroreduction of Carbon Dioxide to Liquid Fuels. Angew. Chemie 2019, 131, 1517815182.

(373) Toney, M. F.; Gordon, J. G.; Samant, M. G.; Borges, G. L.; Melroy, O. R.; Kau, L.-S.; Wiesler, D. G.; Yee, D.; Sorensen, L. B. Surface X-Ray-Scattering Measurements of the Substrate-Induced Spatial Modulation of an Incommensurate Adsorbed Monolayer. Phys. Rev. B 1990, 42, 5594-5603.

(374) Robinson, K. M.; O’Grady, W. E. X-Ray Diffraction and Electrochemical Study on the Oxidation of Flame-Annealed $\mathrm{Au}(100)$ Single-Crystal Surfaces. J. Electroanal. Chem. 1995, 384, 139-144.

(375) Ocko, B. M.; Wang, J.; Davenport, A.; Isaacs, H. In Situ X-Ray Reflectivity and Diffraction Studies of the $\mathrm{Au}(001)$ Reconstruction in an Electrochemical Cell. Phys. Rev. Lett. 1990, 65, 1466-1469.

(376) Magnussen, O. M.; Krug, K.; Ayyad, A. H.; Stettner, J. In Situ Diffraction Studies of Electrode Surface Structure during Gold Electrodeposition. Electrochim. Acta 2008, 53, 3449-3458.

(377) Renner, F. U.; Gründer, Y.; Zegenhagen, J. Portable Chamber for the Study of UHV Prepared Electrochemical Interfaces by Hard X-Ray Diffraction. Rev. Sci. Instrum. 2007, 78, 033903. 
(378) Binninger, T.; Fabbri, E.; Patru, A.; Garganourakis, M.; Han, J.; Abbott, D. F.; Sereda, O.; Kötz, R.; Menzel, A.; Nachtegaal, M.; Schmidt, T. J. Electrochemical Flow-Cell Setup for In Situ X-Ray Investigations. J. Electrochem. Soc. 2016, 163, H906-H912.

(379) Zheng, X.; Zhang, B.; De Luna, P.; Liang, Y.; Comin, R.; Voznyy, O.; Han, L.; García de Arquer, F. P.; Liu, M.; Dinh, C. T.; Regier, T.; Dynes, J. J.; He, S.; Xin, H. L.; Peng, H.; Prendergast, D.; Du, X.; Sargent, E. H. Theory-Driven Design of High-Valence Metal Sites for Water Oxidation Confirmed Using in Situ Soft X-Ray Absorption. Nat. Chem. 2018, 10, 149-154.

(380) Ishihara, T.; Tokushima, T.; Horikawa, Y.; Kato, M.; Yagi, I. Development of a SpectroElectrochemical Cell for Soft X-Ray Photon-in Photon-out Spectroscopy. Rev. Sci. Instrum. 2017, 88, 104101.

(381) Newton, M. A.; Knorpp, A. J.; Meyet, J.; Stoian, D.; Nachtegaal, M.; Clark, A. H.; Safonova, O. V.; Emerich, H.; van Beek, W.; Sushkevich, V. L.; van Bokhoven, J. A. Unwanted Effects of X-Rays in Surface Grafted Copper(II) Organometallics and Copper Exchanged Zeolites, How They Manifest, and What Can Be Done about Them. Phys. Chem. Chem. Phys. 2020, 22, 6826-6837.

(382) de Jonge, N.; Houben, L.; Dunin-Borkowski, R. E.; Ross, F. M. Resolution and Aberration Correction in Liquid Cell Transmission Electron Microscopy. Nat. Rev. Mater. 2019, 4, 61-78.

(383) Dai, S.; You, Y.; Zhang, S.; Cai, W.; Xu, M.; Xie, L.; Wu, R.; Graham, G. W.; Pan, X. In Situ Atomic-Scale Observation of Oxygen-Driven Core-Shell Formation in Pt3Co Nanoparticles. Nat. Commun. 2017, 8, 204.

(384) Dembélé, K.; Bahri, M.; Melinte, G.; Hirlimann, C.; Berliet, A.; Maury, S.; Gay, A.-S.; Ersen, O. Insight by In Situ Gas Electron Microscopy on the Thermal Behaviour and Surface Reactivity of Cobalt Nanoparticles. ChemCatChem 2018, 10, 4004-4009.

(385) Avanesian, T.; Dai, S.; Kale, M. J.; Graham, G. W.; Pan, X.; Christopher, P. Quantitative and Atomic-Scale View of CO-Induced Pt Nanoparticle Surface Reconstruction at Saturation Coverage via DFT Calculations Coupled with in Situ TEM and IR. J. Am. Chem. Soc. 2017, 139, 4551-4558.

(386) Yoshida, K.; Xudong, Z.; Bright, A. N.; Saitoh, K.; Tanaka, N. Dynamic Environmental Transmission Electron Microscopy Observation of Platinum Electrode Catalyst Deactivation in a Proton-Exchange-Membrane Fuel Cell. Nanotechnology 2013, 24, 065705 .

(387) Yoshida, K.; Bright, A. N.; Ward, M. R.; Lari, L.; Zhang, X.; Hiroyama, T.; Boyes, E. 
D.; Gai, P. L. Dynamic Wet-ETEM Observation of Pt/C Electrode Catalysts in a Moisturized Cathode Atmosphere. Nanotechnology 2014, 25, 425702.

(388) Luo, L.; Engelhard, M. H.; Shao, Y.; Wang, C. Revealing the Dynamics of Platinum Nanoparticle Catalysts on Carbon in Oxygen and Water Using Environmental TEM. ACS Catal. 2017, 7, 7658-7664.

(389) Mildner, S.; Beleggia, M.; Mierwaldt, D.; Hansen, T. W.; Wagner, J. B.; Yazdi, S.; Kasama, T.; Ciston, J.; Zhu, Y.; Jooss, C. Environmental TEM Study of Electron Beam Induced Electrochemistry of $\operatorname{Pr} 0.64 \mathrm{Ca} 0.36 \mathrm{MnO} 3$ Catalysts for Oxygen Evolution. $J$. Phys. Chem. C 2015, 119, 5301-5310.

(390) Mierwaldt, D.; Roddatis, V.; Risch, M.; Scholz, J.; Geppert, J.; Abrishami, M. E.; Jooss, C. Environmental TEM Investigation of Electrochemical Stability of Perovskite and Ruddlesden-Popper Type Manganite Oxygen Evolution Catalysts. Adv. Sustain. Syst. 2017, 1, 1700109.

(391) Rehn, S. M.; Jones, M. R. New Strategies for Probing Energy Systems with In Situ Liquid-Phase Transmission Electron Microscopy. ACS Energy Lett. 2018, 3, 12691278 .

(392) Ambrožič, B.; Prašnikar, A.; Hodnik, N.; Kostevšek, N.; Likozar, B.; Rožman, K. Ž.; Šturm, S. Controlling the Radical-Induced Redox Chemistry inside a Liquid-Cell TEM. Chem. Sci. 2019, 10, 8735-8743.

(393) Woehl, T. J.; Abellan, P. Defining the Radiation Chemistry during Liquid Cell Electron Microscopy to Enable Visualization of Nanomaterial Growth and Degradation Dynamics. J. Microsc. 2017, 265, 135-147.

(394) Jungjohann, K. L.; Bliznakov, S.; Sutter, P. W.; Stach, E. A.; Sutter, E. A. In Situ Liquid Cell Electron Microscopy of the Solution Growth of Au-Pd Core-Shell Nanostructures. Nano Lett. 2013, 13, 2964-2970.

(395) Sutter, E.; Jungjohann, K.; Bliznakov, S.; Courty, A.; Maisonhaute, E.; Tenney, S.; Sutter, P. In Situ Liquid-Cell Electron Microscopy of Silver-Palladium Galvanic Replacement Reactions on Silver Nanoparticles. Nat. Commun. 2014, 5, 4946.

(396) Liu, Y.; Lin, X.-M.; Sun, Y.; Rajh, T. In Situ Visualization of Self-Assembly of Charged Gold Nanoparticles. J. Am. Chem. Soc. 2013, 135, 3764-3767.

(397) Chee, S. W.; Tan, S. F.; Baraissov, Z.; Bosman, M.; Mirsaidov, U. Direct Observation of the Nanoscale Kirkendall Effect during Galvanic Replacement Reactions. Nat. Commun. 2017, 8, 1224.

(398) Textor, M.; de Jonge, N. Strategies for Preparing Graphene Liquid Cells for 
Transmission Electron Microscopy. Nano Lett. 2018, 18, 3313-3321.

(399) Hauwiller, M. R.; Ondry, J. C.; Alivisatos, A. P. Using Graphene Liquid Cell Transmission Electron Microscopy to Study in Situ Nanocrystal Etching. J. Vis. Exp. 2018, No. 135, (135).

(400) Williamson, M. J.; Tromp, R. M.; Vereecken, P. M.; Hull, R.; Ross, F. M. Dynamic Microscopy of Nanoscale Cluster Growth at the Solid-Liquid Interface. Nat. Mater. 2003, 2, 532-536.

(401) Toyota, JFCC Breakthrough in Real-Time Observation of Fuel Cell Catalyst Degradation. Fuel Cells Bull. 2015, 2015, 14-15.

(402) Zhu, G.-Z.; Prabhudev, S.; Yang, J.; Gabardo, C. M.; Botton, G. A.; Soleymani, L. In Situ Liquid Cell TEM Study of Morphological Evolution and Degradation of Pt-Fe Nanocatalysts During Potential Cycling. J. Phys. Chem. C 2014, 118, 22111-22119.

(403) Beermann, V.; Holtz, M. E.; Padgett, E.; de Araujo, J. F.; Muller, D. A.; Strasser, P. Real-Time Imaging of Activation and Degradation of Carbon Supported Octahedral PtNi Alloy Fuel Cell Catalysts at the Nanoscale Using in Situ Electrochemical Liquid Cell STEM. Energy Environ. Sci. 2019, 12, 2476-2485.

(404) Ortiz Peña, N.; Ihiawakrim, D.; Han, M.; Lassalle-Kaiser, B.; Carenco, S.; Sanchez, C.; Laberty-Robert, C.; Portehault, D.; Ersen, O. Morphological and Structural Evolution of Co3O4 Nanoparticles Revealed by in Situ Electrochemical Transmission Electron Microscopy during Electrocatalytic Water Oxidation. ACS Nano 2019, 13, 1137211381.

(405) Arán-Ais, R. M.; Rizo, R.; Grosse, P.; Algara-Siller, G.; Dembélé, K.; Plodinec, M.; Lunkenbein, T.; Chee, S. W.; Roldan Cuenya, B. Imaging Electrochemically Synthesized $\mathrm{Cu} 2 \mathrm{O}$ Cubes and Their Morphological Evolution under Conditions Relevant to CO2 Electroreduction. Nat. Commun. 2020, 11, 3489.

(406) Li, W.; Chen, Z.; Xu, L.; Yan, Y. A Solution-Phase Synthesis Method to Highly Active $\mathrm{Pt}-\mathrm{Co} / \mathrm{C}$ Electrocatalysts for Proton Exchange Membrane Fuel Cell. J. Power Sources 2010, 195, 2534-2540.

(407) Stamenkovic, V. R.; Markovic, N. M. Tailored High-Performance Low-PlatinumGroup-Metal Alloy Cathode Catalysts. DOE Hydrog. Fuel Cells Program, FY 2019 Annu. Prog. Rep. 2019, 1-7.

(408) Dionigi, F.; Weber, C. C.; Primbs, M.; Gocyla, M.; Bonastre, A. M.; Spöri, C.; Schmies, H.; Hornberger, E.; Kühl, S.; Drnec, J.; Heggen, M.; Sharman, J.; Dunin-Borkowski, R. E.; Strasser, P. Controlling Near-Surface Ni Composition in Octahedral PtNi(Mo) 
Nanoparticles by Mo Doping for a Highly Active Oxygen Reduction Reaction Catalyst. Nano Lett. 2019, 19, 6876-6885.

(409) Han, B.; Carlton, C. E.; Kongkanand, A.; Kukreja, R. S.; Theobald, B. R.; Gan, L.; O’Malley, R.; Strasser, P.; Wagner, F. T.; Shao-Horn, Y. Record Activity and Stability of Dealloyed Bimetallic Catalysts for Proton Exchange Membrane Fuel Cells. Energy Environ. Sci. 2015, 8, 258-266.

(410) Pan, L.; Ott, S.; Dionigi, F.; Strasser, P. Current Challenges Related to the Deployment of Shape-Controlled Pt Alloy Oxygen Reduction Reaction Nanocatalysts into Low PtLoaded Cathode Layers of Proton Exchange Membrane Fuel Cells. Curr. Opin. Electrochem. 2019, 18, 61-71.

(411) Yarlagadda, V.; Carpenter, M. K.; Moylan, T. E.; Kukreja, R. S.; Koestner, R.; Gu, W.; Thompson, L.; Kongkanand, A. Boosting Fuel Cell Performance with Accessible Carbon Mesopores. ACS Energy Lett. 2018, 3, 618-621.

(412) Kongkanand, A.; Subramanian, N. P.; Yu, Y.; Liu, Z.; Igarashi, H.; Muller, D. A. Achieving High-Power PEM Fuel Cell Performance with an Ultralow-Pt-Content CoreShell Catalyst. ACS Catal. 2016, 6, 1578-1583.

(413) Kongkanand, A.; Mathias, M. F. The Priority and Challenge of High-Power Performance of Low-Platinum Proton-Exchange Membrane Fuel Cells. J. Phys. Chem. Lett. 2016, 7, 1127-1137.

(414) Schuler, T.; Chowdhury, A.; Freiberg, A. T.; Sneed, B.; Spingler, F. B.; Tucker, M. C.; More, K. L.; Radke, C. J.; Weber, A. Z. Fuel-Cell Catalyst-Layer Resistance via Hydrogen Limiting-Current Measurements. J. Electrochem. Soc. 2019, 166, F3020F3031.

(415) Martens, S.; Asen, L.; Ercolano, G.; Dionigi, F.; Zalitis, C.; Hawkins, A.; Martinez Bonastre, A.; Seidl, L.; Knoll, A. C.; Sharman, J.; Strasser, P.; Jones, D.; Schneider, O. A Comparison of Rotating Disc Electrode, Floating Electrode Technique and Membrane Electrode Assembly Measurements for Catalyst Testing. J. Power Sources 2018, 392, 274-284.

(416) De Gregorio, G. L.; Burdyny, T.; Loiudice, A.; Iyengar, P.; Smith, W. A.; Buonsanti, R. Facet-Dependent Selectivity of Cu Catalysts in Electrochemical CO2 Reduction at Commercially Viable Current Densities. ACS Catal. 2020, 10, 4854-4862.

(417) Schouten, K. J. P.; Pérez Gallent, E.; Koper, M. T. M. The Influence of PH on the Reduction of $\mathrm{CO}$ and $\mathrm{CO} 2$ to Hydrocarbons on Copper Electrodes. J. Electroanal. Chem. 2014, 716, 53-57. 
(418) Dinh, C.-T.; Burdyny, T.; Kibria, M. G.; Seifitokaldani, A.; Gabardo, C. M.; García de Arquer, F. P.; Kiani, A.; Edwards, J. P.; De Luna, P.; Bushuyev, O. S.; Zou, C.; Quintero-Bermudez, R.; Pang, Y.; Sinton, D.; Sargent, E. H. CO2 Electroreduction to Ethylene via Hydroxide-Mediated Copper Catalysis at an Abrupt Interface. Science 2018, 360, 783-787.

(419) Wang, Y.; Shen, H.; Livi, K. J. T.; Raciti, D.; Zong, H.; Gregg, J.; Onadeko, M.; Wan, Y.; Watson, A.; Wang, C. Copper Nanocubes for CO2 Reduction in Gas Diffusion Electrodes. Nano Lett. 2019, 19, 8461-8468.

(420) Möller, T.; Scholten, F.; Thanh, T. N.; Sinev, I.; Timoshenko, J.; Wang, X.; Jovanov, Z.; Gliech, M.; Roldan Cuenya, B.; Varela, A. S.; Strasser, P. Electrocatalytic CO2 Reduction on CuOx Nanocubes: Tracking the Evolution of Chemical State, Geometric Structure, and Catalytic Selectivity Using Operando Spectroscopy. Angew. Chemie Int. Ed. 2020, 59, 17974-17983.

(421) Yang, X.; De Andrade, V.; Scullin, W.; Dyer, E. L.; Kasthuri, N.; De Carlo, F.; Gürsoy, D. Low-Dose x-Ray Tomography through a Deep Convolutional Neural Network. Sci. Rep. 2018, 8, 2575.

(422) Villanova, J.; Daudin, R.; Lhuissier, P.; Jauffrès, D.; Lou, S.; Martin, C. L.; Labouré, S.; Tucoulou, R.; Martínez-Criado, G.; Salvo, L. Fast in Situ 3D Nanoimaging: A New Tool for Dynamic Characterization in Materials Science. Mater. Today 2017, 20, 354-359.

(423) Normile, S. J.; Zenyuk, I. V. Imaging Ionomer in Fuel Cell Catalyst Layers with Synchrotron Nano Transmission X-Ray Microscopy. Solid State Ionics 2019, 335, 3846.

(424) Lim, J.; Li, Y.; Alsem, D. H.; So, H.; Lee, S. C.; Bai, P.; Cogswell, D. A.; Liu, X.; Jin, N.; Yu, Y.; Salmon, N. J.; Shapiro, D. A.; Bazant, M. Z.; Tyliszczak, T.; Chueh, W. C. Origin and Hysteresis of Lithium Compositional Spatiodynamics within Battery Primary Particles. Science 2016, 353, 566-571.

(425) Meyer, Q.; Mansor, N.; Iacoviello, F.; Cullen, P. L.; Jervis, R.; Finegan, D.; Tan, C.; Bailey, J.; Shearing, P. R.; Brett, D. J. L. Investigation of Hot Pressed Polymer Electrolyte Fuel Cell Assemblies via X-Ray Computed Tomography. Electrochim. Acta 2017, 242, 125-136.

(426) Pokhrel, A.; El Hannach, M.; Orfino, F. P.; Dutta, M.; Kjeang, E. Failure Analysis of Fuel Cell Electrodes Using Three-Dimensional Multi-Length Scale X-Ray Computed Tomography. J. Power Sources 2016, 329, 330-338.

(427) Ince, U. U.; Markötter, H.; Ge, N.; Klages, M.; Haußmann, J.; Göbel, M.; Scholta, J.; 
Bazylak, A.; Manke, I. 3D Classification of Polymer Electrolyte Membrane Fuel Cell Materials from In-Situ X-Ray Tomographic Datasets. Int. J. Hydrogen Energy 2020, 45, 12161-12169.

(428) Harry, K. J.; Hallinan, D. T.; Parkinson, D. Y.; MacDowell, A. A.; Balsara, N. P. Detection of Subsurface Structures underneath Dendrites Formed on Cycled Lithium Metal Electrodes. Nat. Mater. 2014, 13, 69-73.

(429) Ebner, M.; Marone, F.; Stampanoni, M.; Wood, V. Visualization and Quantification of Electrochemical and Mechanical Degradation in Li Ion Batteries. Science 2013, 342, $716-720$.

(430) Ziesche, R. F.; Arlt, T.; Finegan, D. P.; Heenan, T. M. M.; Tengattini, A.; Baum, D.; Kardjilov, N.; Markötter, H.; Manke, I.; Kockelmann, W.; Brett, D. J. L.; Shearing, P. R. 4D Imaging of Lithium-Batteries Using Correlative Neutron and X-Ray Tomography with a Virtual Unrolling Technique. Nat. Commun. 2020, 11, 777.

(431) Dong, K.; Osenberg, M.; Sun, F.; Markötter, H.; Jafta, C. J.; Hilger, A.; Arlt, T.; Banhart, J.; Manke, I. Non-Destructive Characterization of Lithium Deposition at the $\mathrm{Li} /$ Separator and Li/Carbon Matrix Interregion by Synchrotron X-Ray Tomography. Nano Energy 2019, 62, 11-19.

(432) Lal, S.; Lamibrac, A.; Eller, J.; Büchi, F. N. Determination of Water Evaporation Rates in Gas Diffusion Layers of Fuel Cells. J. Electrochem. Soc. 2018, 165, F652-F661.

(433) Schuler, T.; De Bruycker, R.; Schmidt, T. J.; Büchi, F. N. Polymer Electrolyte Water Electrolysis: Correlating Porous Transport Layer Structural Properties and Performance: Part I. Tomographic Analysis of Morphology and Topology. J. Electrochem. Soc. 2019, 166, F270-F281.

(434) Schuler, T.; Schmidt, T. J.; Büchi, F. N. Polymer Electrolyte Water Electrolysis: Correlating Performance and Porous Transport Layer Structure: Part II. Electrochemical Performance Analysis. J. Electrochem. Soc. 2019, 166, F555-F565.

(435) Wu, J.; Melo, L. G. A.; Zhu, X.; West, M. M.; Berejnov, V.; Susac, D.; Stumper, J.; Hitchcock, A. P. 4D Imaging of Polymer Electrolyte Membrane Fuel Cell Catalyst Layers by Soft X-Ray Spectro-Tomography. J. Power Sources 2018, 381, 72-83.

(436) Sun, Y.; Wang, Y. Monitoring of Galvanic Replacement Reaction between Silver Nanowires and HAuCl4 by In Situ Transmission X-Ray Microscopy. Nano Lett. 2011, $11,4386-4392$.

(437) Yu, L.; Yan, Z.; Cai, Z.; Zhang, D.; Han, P.; Cheng, X.; Sun, Y. Quantitatively in Situ Imaging Silver Nanowire Hollowing Kinetics. Nano Lett. 2016, 16, 6555-6559. 
(438) Cats, K. H.; Andrews, J. C.; Stéphan, O.; March, K.; Karunakaran, C.; Meirer, F.; de Groot, F. M. F.; Weckhuysen, B. M. Active Phase Distribution Changes within a Catalyst Particle during Fischer-Tropsch Synthesis as Revealed by Multi-Scale Microscopy. Catal. Sci. Technol. 2016, 6, 4438-4449.

(439) Harris, W. M.; Lombardo, J. J.; Nelson, G. J.; Lai, B.; Wang, S.; Vila-Comamala, J.; Liu, M.; Liu, M.; Chiu, W. K. S. Three-Dimensional Microstructural Imaging of Sulfur Poisoning-Induced Degradation in a Ni-YSZ Anode of Solid Oxide Fuel Cells. Sci. Rep. 2015, 4, 5246.

(440) Chao, W.; Harteneck, B. D.; Liddle, J. A.; Anderson, E. H.; Attwood, D. T. Soft X-Ray Microscopy at a Spatial Resolution Better than 15 Nm. Nature 2005, 435, 1210-1213.

(441) Chen-Wiegart, Y. K.; DeMike, R.; Erdonmez, C.; Thornton, K.; Barnett, S. A.; Wang, J. Tortuosity Characterization of 3D Microstructure at Nano-Scale for Energy Storage and Conversion Materials. J. Power Sources 2014, 249, 349-356.

(442) Matsuyama, S.; Yasuda, S.; Yamada, J.; Okada, H.; Kohmura, Y.; Yabashi, M.; Ishikawa, T.; Yamauchi, K. 50-Nm-Resolution Full-Field X-Ray Microscope without Chromatic Aberration Using Total-Reflection Imaging Mirrors. Sci. Rep. 2017, 7, 46358.

(443) Wood, V. X-Ray Tomography for Battery Research and Development. Nat. Rev. Mater. 2018, 3, 293-295.

(444) Yang, K.; Kas, R.; Smith, W. A.; Burdyny, T. Role of the Carbon-Based Gas Diffusion Layer on Flooding in a Gas Diffusion Electrode Cell for Electrochemical CO2 Reduction. ACS Energy Lett. 2020, 33-40.

(445) Jhong, H.-R. "Molly"; Brushett, F. R.; Kenis, P. J. A. The Effects of Catalyst Layer Deposition Methodology on Electrode Performance. Adv. Energy Mater. 2013, 3, 589599.

(446) Bredol, M.; Szydło, A.; Radev, I.; Philippi, W.; Bartholomäus, R.; Peinecke, V.; Heinzel, A. How the Colloid Chemistry of Precursor Electrocatalyst Dispersions Is Related to the Polymer Electrolyte Membrane Fuel Cell Performance. J. Power Sources 2018, 402, 15-23.

(447) Szydło, A.; Goossen, J.-D.; Linte, C.; Uphoff, H.; Bredol, M. Preparation of PlatinumBased Electrocatalytic Layers from Catalyst Dispersions with Adjusted Colloidal Stability via a Pulsed Electrophoretic Deposition Method. Mater. Chem. Phys. 2020, $242,122532$.

(448) Mehta, V.; Cooper, J. S. Review and Analysis of PEM Fuel Cell Design and 
Manufacturing. J. Power Sources 2003, 114, 32-53.

(449) Suter, S.; Haussener, S. Optimizing Mesostructured Silver Catalysts for Selective Carbon Dioxide Conversion into Fuels. Energy Environ. Sci. 2019, 12, 1668-1678.

(450) Zhang, L.; Xia, Y. Scaling up the Production of Colloidal Nanocrystals: Should We Increase or Decrease the Reaction Volume? Adv. Mater. 2014, 26, 2600-2606.

(451) Zhang, L.; Niu, G.; Lu, N.; Wang, J.; Tong, L.; Wang, L.; Kim, M. J.; Xia, Y. Continuous and Scalable Production of Well-Controlled Noble-Metal Nanocrystals in MilliliterSized Droplet Reactors. Nano Lett. 2014, 14, 6626-6631.

(452) Baddour, F. G.; Roberts, E. J.; To, A. T.; Wang, L.; Habas, S. E.; Ruddy, D. A.; Bedford, N. M.; Wright, J.; Nash, C. P.; Schaidle, J. A.; Brutchey, R. L.; Malmstadt, N. An Exceptionally Mild and Scalable Solution-Phase Synthesis of Molybdenum Carbide Nanoparticles for Thermocatalytic CO2 Hydrogenation. J. Am. Chem. Soc. 2020, 142, 1010-1019.

(453) Weckhuysen, B. M. Preface: Recent Advances in the in-Situ Characterization of Heterogeneous Catalysts. Chem. Soc. Rev. 2010, 39, 4557.

(454) Grey, C. P.; Dupré, N. NMR Studies of Cathode Materials for Lithium-Ion Rechargeable Batteries. Chem. Rev. 2004, 104, 4493-4512.

(455) Hanrahan, M. P.; Chen, Y.; Blome-Fernández, R.; Stein, J. L.; Pach, G. F.; Adamson, M. A. S.; Neale, N. R.; Cossairt, B. M.; Vela, J.; Rossini, A. J. Probing the Surface Structure of Semiconductor Nanoparticles by DNP SENS with Dielectric Support Materials. J. Am. Chem. Soc. 2019, 141, 15532-15546.

(456) Al-Johani, H.; Abou-Hamad, E.; Jedidi, A.; Widdifield, C. M.; Viger-Gravel, J.; Sangaru, S. S.; Gajan, D.; Anjum, D. H.; Ould-Chikh, S.; Hedhili, M. N.; Gurinov, A.; Kelly, M. J.; El Eter, M.; Cavallo, L.; Emsley, L.; Basset, J.-M. The Structure and Binding Mode of Citrate in the Stabilization of Gold Nanoparticles. Nat. Chem. 2017, 9, 890-895.

(457) Zhao, M.; Xia, Y. Crystal-Phase and Surface-Structure Engineering of Ruthenium Nanocrystals. Nat. Rev. Mater. 2020, 5, 440-459.

(458) Koziej, D. Revealing Complexity of Nanoparticle Synthesis in Solution by in Situ Hard X-Ray Spectroscopy-Today and Beyond. Chem. Mater. 2016, 28, 2478-2490.

(459) Wu, S.; Li, M.; Sun, Y. In Situ Synchrotron X-ray Characterization Shining Light on the Nucleation and Growth Kinetics of Colloidal Nanoparticles. Angew. Chemie Int. Ed. 2019, 58, 8987-8995.

(460) Minteer, S. D.; Baran, P. Electrifying Synthesis: Recent Advances in the Methods, 
Materials, and Techniques for Organic Electrosynthesis. Acc. Chem. Res. 2020, 53, 545546.

(461) Singh, A. R.; Rohr, B. A.; Schwalbe, J. A.; Cargnello, M.; Chan, K.; Jaramillo, T. F.; Chorkendorff, I.; Nørskov, J. K. Electrochemical Ammonia Synthesis-The Selectivity Challenge. ACS Catal. 2017, 7, 706-709. 


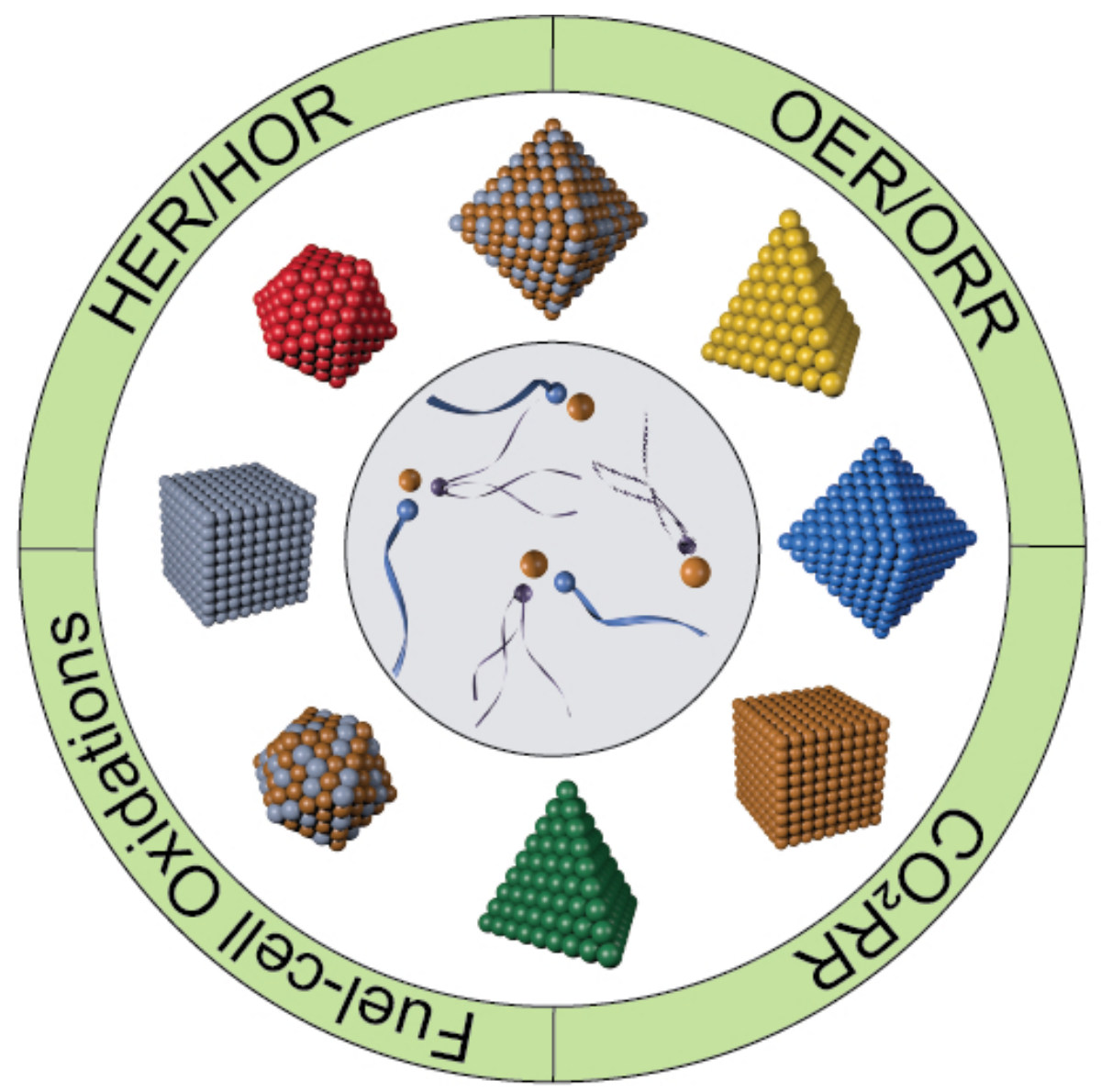

